\title{
Natural Derivations for Priest, An Introduction to Non-Classical Logic
}

\author{
TONy Roy* \\ Department of Philosophy \\ California State University, San Bernardino \\ troy@csusb.edu \\ Received by Greg Restall \\ Published March 20, 2006 \\ http://www ·philosophy · unimelb . edu . au/aj1/2006 \\ (C) 2006 Tony Roy
}

\begin{abstract}
A вstract: This document collects natural derivation systems for logics described in Priest, An Introduction to Non-Classical Logic [4]. It provides an alternative or supplement to the semantic tableaux of his text. Except that some chapters are collapsed, there are sections for each chapter in Priest, with an additional, final section on quantified modal logic. In each case, (i) the language is briefly described and key semantic definitions stated, (ii) the derivation system is presented with a few examples given, and (iii) soundness and completeness are proved. There should be enough detail to make the parts accessible to students would work through parallel sections of Priest.
\end{abstract}

This document collects natural derivation systems for logics described in Priest, An Introduction to Non-Classical Logic [4]. It thus provides an alternative or supplement to the semantic tableaux of his text. Some of the derivation systems may also be of interest in their own right. They are all Fitch-style systems on the model of [I, I2], and many other places. Though a classical system is presented for chapter I, prior acquaintance with some such system is assumed. Associated goal-directed derivation strategies are discussed extensively in [I2, chapter 6].

Except that some chapters are collapsed, there are sections for each chapter in Priest, with an additional, final section on quantified modal logic. In each

*Thanks to Graham Priest for encouragement, and to referee(s) for this fournal who took the time to plow through the details, and make many helpful suggestions. Thanks also to Darcy Otto for getting me started on $\mathrm{LT}_{\mathrm{E}} \mathrm{X}$, with which this document was produced. 
case, (i) the language is briefly described and key semantic definitions stated, (ii) the derivation system is presented with a few examples given, and (iii) soundness and completeness are proved. Notation is common with Priest, though some nomenclature is improvised to keep things systematic. Though cases of some proofs are left to the reader, there should be enough detail to make the parts accessible to students who would work through parallel sections of Priest.

Demonstrations of completeness are all on the model of the standard argument for classical logic, and simplified considerably by the use of "subscripts" and "overlines" in derivations. For the most part, I take over approaches from tableaux in Priest. Thus, e.g., subscripts are like indexes from his tableaux. Overlines are like underlines in [13]. Advantages of the approach to completeness are particularly dramatic when quantifiers are introduced, as exhibited in the section on quantified modal logic.

\section{TABLE OF CONTENTS}

I CLASSICAL LOGIC: CL (CH. I) 48

2 NORMAL MOdAl LOGiCS: $K \alpha(\mathrm{CH}, 2,3) \quad 56$



4 COnditional logics: $C x(\mathrm{CH} .5) \quad 84$

5 Intuitionistic LOGiC: $I L($ CH. 6) IOO

6 MANY-Valued LOGiCS: $M x(\mathrm{CH} .7,8)$

7 BASIC RELEVANT LOGIC: $v X(\mathrm{CH} .9) \quad$ I25

8 MAinstream RELEVANT LOGICS: $B x$ (CH. IO,II) I4I

9 QuAntified Modal LOGiCs: Fn $\alpha \quad$ I64

I CLASSICAL LOGIC: $C L(\mathrm{CH} . \mathrm{I})$

I.I LANGUAGE / SEMANTIC NOTIONS

LCL The LANGUage consists of propositional parameters $p_{0}, p_{1} \ldots$ combined in the usual way with the operators, $\neg, \wedge, \vee, \supset$, and $\equiv$. So each propositional parameter is a FORMULA; if $A$ and $B$ are formulas, so are $\neg A,(A \wedge B),(A \vee B),(A \supset B)$ and $(A \equiv B)$.

icl An interpretation is a function $v$ which assigns to each propositional parameter either I (true) or o (false). 
TCL For complex expressions,

$(\neg) \quad v(\neg A)=1$ if $v(A)=0$, and 0 otherwise.

$(\wedge) v(A \wedge B)=1$ if $v(A)=1$ and $v(B)=1$, and 0 otherwise.

( $\vee v(A \vee B)=1$ if $v(A)=1$ or $v(B)=1$, and 0 otherwise.

(つ) $v(A \supset B)=1$ if $v(A)=0$ or $v(B)=1$, and 0 otherwise.

$(\equiv) v(\mathrm{~A} \equiv \mathrm{B})=1$ if $v(\mathrm{~A})=v(\mathrm{~B})$, and 0 otherwise.

For a set $\Gamma$ of formulas, $v(\Gamma)=1 \operatorname{iff} v(A)=1$ for each $A \in \Gamma$; then,

$\operatorname{vCL} \Gamma \models_{\mathrm{CL}} A$ iff there is no $C L$ interpretation $v$ such that $v(\Gamma)=1$ and $v(A)=$ 0.

\section{I.2 NATURAL DERIVATIONS: NCL}

$N C L$ is just the sentential portion of the system $N D$ from [12, chapter 6]. Refer to that source for examples and further discussion (compare, e.g., [I]). Every line of a derivation is a premise, an assumption, or justified from previous lines by a rule. The rules include introduction and exploitation rules for each operator, and reiteration. In the parenthetical "exit strategy" for assumptions, ' $c$ ' indicates a contradiction is to be sought, ' $g$ ' a goal at the bottom of the scope line.

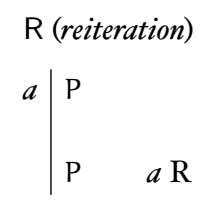

$\wedge \mathrm{I}$ (conjunction intro)

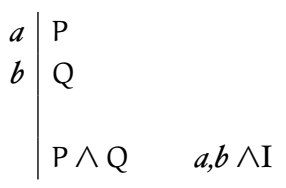

VI (disjunction intro)



I (conditional intro)



$\neg$ (negation intro)

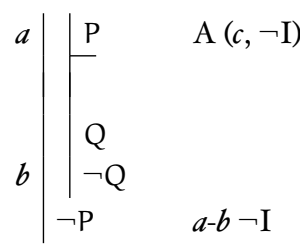

$\wedge \mathrm{E}$ (conjunction exploit)

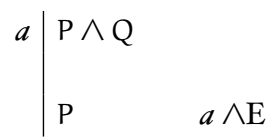

VI (disjunction intro)

$a \mid \mathrm{P}$

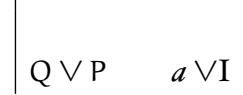

$\supset \mathrm{E}$ (conditional exploit)



$\neg \mathrm{E}$ (negation exploit)

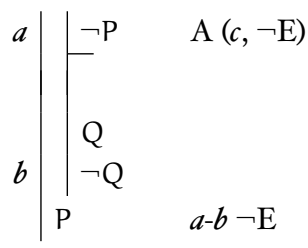

$\wedge \mathrm{E}$ (conjunction exploit)

$a \mid \begin{array}{ll}\mathrm{P} \wedge \mathrm{Q} & \\ \mathrm{Q} & a \wedge \mathrm{E}\end{array}$

VE (disjunction exploit)

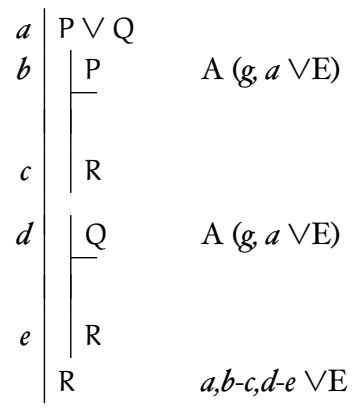




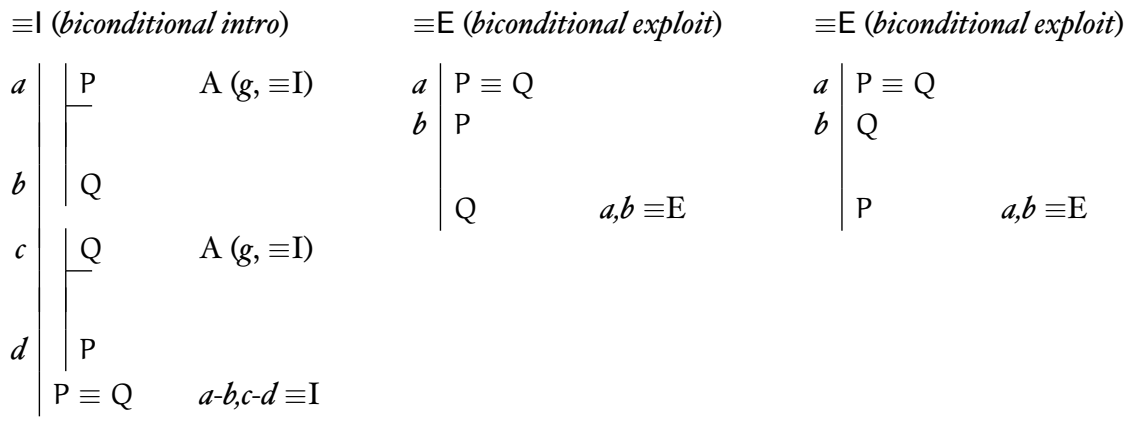

NCL $\Gamma \vdash_{\text {NCL }} A$ iff there is an NCL derivation of $A$ from the members of $\Gamma$.

As derived rules, we accept the following "ordinary" and "two-way" rules. The "two-way" rules are usually presented as replacement rules. Insofar as we will not have much call to use then that way, in order to streamline demonstrations of soundness, we treat them just as ordinary rules which work in either direction - where it is trivial that the rules are in fact derived in this sense from the rules of $N C L$.

Ordinary Derived Rules
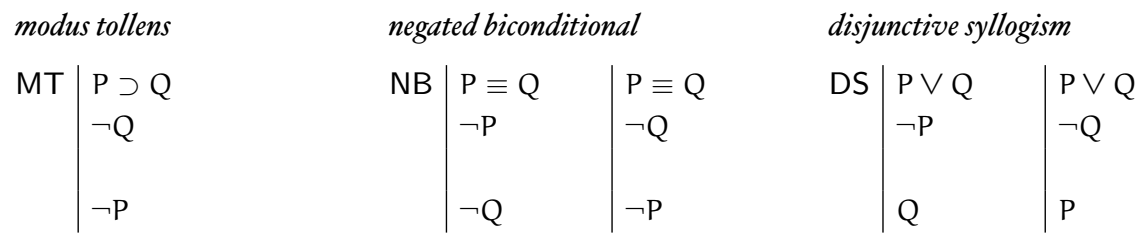

Two-way Derived Rules

$\begin{array}{lll}\text { DN } & \mathrm{P} \triangleleft \triangleright \neg \neg \mathrm{P} & \text { double negation } \\ \text { Com } & \mathrm{P} \wedge \mathrm{Q} \triangleleft \triangleright \mathrm{Q} \wedge \mathrm{P} & \text { commutation } \\ & \mathrm{P} \vee \mathrm{Q} \triangleleft \triangleright \mathrm{Q} \vee \mathrm{P} & \\ \text { Assoc } & \mathrm{P} \wedge(\mathrm{Q} \wedge \mathrm{R}) \triangleleft \triangleright(\mathrm{P} \wedge \mathrm{Q}) \wedge \mathrm{R} & \text { association } \\ & \mathrm{P} \vee(\mathrm{Q} \vee \mathrm{R}) \triangleleft \triangleright(\mathrm{P} \vee \mathrm{Q}) \vee \mathrm{R} & \\ \text { Idem } & \mathrm{P} \triangleleft \triangleright \mathrm{P} \wedge \mathrm{P} & \text { idempotence } \\ & \mathrm{P} \triangleleft \triangleright \mathrm{P} \vee \mathrm{P} & \\ \text { Impl } & \mathrm{P} \supset \mathrm{Q} \triangleleft \triangleright \neg \mathrm{P} \vee \mathrm{Q} & \text { implication } \\ & \neg \mathrm{P} \supset \mathrm{Q} \triangleleft \triangleright \mathrm{P} \vee \mathrm{Q} & \text { transposition } \\ \text { Trans } & \mathrm{P} \supset \mathrm{Q} \triangleleft \triangleright \neg \mathrm{Q} \supset \neg \mathrm{P} & \text { De Morgan } \\ \text { DeM } & \neg(\mathrm{P} \wedge \mathrm{Q}) \triangleleft \triangleright \neg \mathrm{P} \vee \neg \mathrm{Q} & \\ & \neg(\mathrm{P} \vee \mathrm{Q}) \triangleleft \triangleright \neg \mathrm{P} \wedge \neg \mathrm{Q} & \text { exportation } \\ \text { Exp } & \mathrm{P} \supset(\mathrm{Q} \supset \mathrm{R}) \triangleleft \triangleright(\mathrm{P} \wedge \mathrm{Q}) \supset \mathrm{R} & \end{array}$




$\begin{array}{llll}\text { Equiv } & \mathrm{P} \equiv \mathrm{Q} \triangleleft \triangleright(\mathrm{P} \supset \mathrm{Q}) \wedge(\mathrm{Q} \supset \mathrm{P}) & \text { equivalence } \\ & \mathrm{P} \equiv \mathrm{Q} \triangleleft \triangleright(\mathrm{P} \wedge \mathrm{Q}) \vee(\neg \mathrm{P} \wedge \neg \mathrm{Q}) & \\ \text { Dist } & \mathrm{P} \wedge(\mathrm{Q} \vee \mathrm{R}) \triangleleft \triangleright(\mathrm{P} \wedge \mathrm{Q}) \vee(\mathrm{P} \wedge \mathrm{R}) & \text { distribution } \\ & \mathrm{P} \vee(\mathrm{Q} \wedge \mathrm{R}) & \triangleleft \triangleright(\mathrm{P} \vee \mathrm{Q}) \wedge(\mathrm{P} \vee \mathrm{R}) & \end{array}$

As examples, here are derivations to demonstrate the first form of Impl (among the relatively difficult of derivations for the derived rules).

\begin{tabular}{|c|c|c|c|c|c|}
\hline \multicolumn{3}{|c|}{$\neg \mathrm{P} \vee \mathrm{Q} \vdash_{\mathrm{NCL}} \mathrm{P} \supset \mathrm{Q}$} & \multicolumn{3}{|c|}{$\mathrm{P} \supset \mathrm{Q} \vdash_{\mathrm{NCL}} \neg \mathrm{P} \vee \mathrm{Q}$} \\
\hline I & $\neg \mathrm{P} \vee \mathrm{Q}$ & $\mathrm{P}$ & I & $P \supset Q$ & $\mathrm{P}$ \\
\hline 2 & $\neg \mathrm{P}$ & $\mathrm{A}(g, \mathrm{I} \vee \mathrm{E})$ & 2 & $\neg(\neg P \vee Q)$ & $\mathrm{A}(c, \neg \mathrm{E})$ \\
\hline 3 & $P$ & $\mathrm{~A}(g, \supset \mathrm{I})$ & 3 & $P$ & $\mathrm{~A}(c, \neg \mathrm{I})$ \\
\hline 4 & $\neg \mathrm{Q}$ & $\mathrm{A}(c, \neg \mathrm{E})$ & 4 & $\mathrm{Q}$ & $\mathrm{I}, 3 \supset \mathrm{E}$ \\
\hline 5 & $\neg P$ & $2 \mathrm{R}$ & 5 & $\neg \mathrm{P} \vee \mathrm{Q}$ & $4 \vee \mathrm{I}$ \\
\hline 6 & $P$ & $3 \mathrm{R}$ & 6 & $\mid \neg(\neg \mathrm{P} \vee \mathrm{Q})$ & $2 \mathrm{R}$ \\
\hline 7 & Q & $4^{-6} \neg \mathrm{E}$ & 7 & $\neg \mathrm{P}$ & 3-6 ᄀI \\
\hline 8 & $\mathrm{P} \supset \mathrm{Q}$ & 3-7 $\supset \mathrm{I}$ & 8 & $\neg \mathrm{P} \vee \mathrm{Q}$ & $7 \vee \mathrm{I}$ \\
\hline 9 & Q & $\mathrm{A}(g, \mathrm{I} \vee \mathrm{E})$ & IO & $\neg \mathrm{P} \vee \mathrm{Q}$ & $2-9 \neg E$ \\
\hline IO & $P$ & $\mathrm{~A}(g, \supset \mathrm{I})$ & & & \\
\hline II & Q & $9 \mathrm{R}$ & & & \\
\hline $\mathrm{I} 2$ & $P \supset Q$ & IO-II $\supset$ I & & & \\
\hline 13 & $P \supset Q$ & $\mathrm{I}, 2-8,9-\mathrm{I} 2 \vee \mathrm{E}$ & & & \\
\hline
\end{tabular}

\section{I.3 SOUNDNESS AND COMPLETENESS}

The following are standard arguments. Cases that are omitted are like ones worked, and so left to the reader.

THEOREM I.I NCL is sound: If $\Gamma \vdash_{\mathrm{NCL}}$ A then $\Gamma \models_{\mathrm{CL}}$ A.

Li.I If $\Gamma \subseteq \Gamma^{\prime}$ and $\Gamma \models_{\mathrm{CL}} \mathrm{P}$, then $\Gamma^{\prime} \models_{\mathrm{CL}}$ P.

Suppose $\Gamma \subseteq \Gamma^{\prime}$ and $\Gamma \models_{\mathrm{CL}} \mathrm{P}$, but $\Gamma^{\prime} \not \nvdash_{\mathrm{CL}} \mathrm{P}$. From the latter, by VCL, there is some $v$ such that $v\left(\Gamma^{\prime}\right)=1$ but $v(\mathrm{P})=0$. But since $v\left(\Gamma^{\prime}\right)=1$ and $\Gamma \subseteq \Gamma^{\prime}, v(\Gamma)=1$; so $v$ is a $C L$ interpretation such that $v(\Gamma)=1$ but $v(\mathrm{P})=0$; so by VCL, $\Gamma \nvdash_{\mathrm{CL}} \mathrm{P}$. This is impossible; reject the assumption: if $\Gamma \subseteq \Gamma^{\prime}$ and $\Gamma \models_{\mathrm{CL}} \mathrm{P}$, then $\Gamma^{\prime} \models_{\mathrm{CL}} \mathrm{P}$.

Main result: For each line in a derivation let $A_{i}$ be the formula on line $i$ and set $\Gamma_{i}$ equal to the set of all premises and assumptions whose scope includes line $i$. Suppose $\Gamma \vdash_{\mathrm{NCL}} A$. Then there is a derivation of $A$ from premises in $\Gamma$ where $A$ appears under the scope of the premises alone. By induction on line number of this derivation, we show that for each line $i$ of this derivation, $\Gamma_{i} \models_{C L} A_{i}$. The case when $A_{i}=A$ is the desired result. 
Basis: $A_{1}$ is a premise or an assumption. Then $\Gamma_{1}=\left\{A_{1}\right\}$; so $v\left(\Gamma_{1}\right)=1$ iff $v\left(A_{1}\right)=1$; so there is no $v$ such that $v\left(\Gamma_{1}\right)=1$ but $v\left(A_{1}\right)=0$. So by VCL, $\Gamma_{1} \models_{\mathrm{CL}} A_{1}$.

Assp: For any $i, 1 \leqslant i<k, \Gamma_{i} \models_{C L} A_{i}$.

Show: $\Gamma_{\mathrm{k}} \models_{\mathrm{CL}} A_{\mathrm{k}}$.

$A_{k}$ is either a premise, an assumption, or arises from previous lines by $\mathrm{R}, \supset \mathrm{I}, \supset \mathrm{E}, \wedge \mathrm{I}, \wedge \mathrm{E}, \neg \mathrm{I}, \neg \mathrm{E}, \vee \mathrm{I}, \vee \mathrm{E}, \equiv \mathrm{I}$ or $\equiv \mathrm{E}$. If $A_{k}$ is a premise or an assumption, then as in the basis, $\Gamma_{k} \models_{C L} A_{k}$. So suppose $A_{k}$ arises by one of the rules.

(R)

( $\supset$ I) If $A_{k}$ arises by $\supset \mathrm{I}$, then the picture is like this,

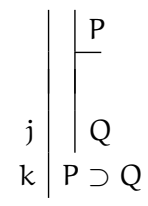

where $j<k$ and $A_{k}$ is $P \supset Q$. By assumption, $\Gamma_{j} \models_{C L} Q$; and by the nature of access, $\Gamma_{j} \subseteq \Gamma_{k} \cup\{P\}$; so by LI.I, $\Gamma_{k} \cup\{P\} \models_{C L} Q$. Suppose $\Gamma_{\mathrm{k}} \not \forall_{\mathrm{CL}} \mathrm{P} \supset \mathrm{Q}$; then by VCL, there is some $v$ such that $v\left(\Gamma_{\mathrm{k}}\right)=1$ but $v(\mathrm{P} \supset \mathrm{Q})=0$; from the latter, by $\mathrm{TCL}(\supset), v(\mathrm{P})=1$ and $v(\mathrm{Q})=0$; so $v\left(\Gamma_{k}\right)=1$ and $v(\mathrm{P})=1$; so $v\left(\Gamma_{k} \cup\{\mathrm{P}\}\right)=1$; so by VCL, $v(\mathrm{Q})=1$. This is impossible; reject the assumption: $\Gamma_{\mathrm{K}} \models_{\mathrm{CL}} \mathrm{P} \supset \mathrm{Q}$, which is to say, $\Gamma_{\mathrm{k}} \models_{\mathrm{CL}} A_{\mathrm{k}}$.

( $\supset \mathrm{E})$ If $A_{k}$ arises by $\supset \mathrm{E}$, then the picture is like this,

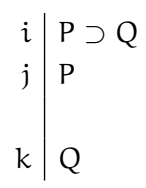

where $i, j<k$ and $A_{k}$ is $Q$. By assumption, $\Gamma_{i} \models_{C L} P \supset Q$ and $\Gamma_{j} \models_{C L} P$; but by the nature of access, $\Gamma_{i} \subseteq \Gamma_{k}$ and $\Gamma_{j} \subseteq \Gamma_{k}$; so by LI.I, $\Gamma_{k} \models_{C L} \mathrm{P} \supset \mathrm{Q}$ and $\Gamma_{k} \models_{C L} P$. Suppose $\Gamma_{k} \forall_{C L}$ Q; then by VCL, there is some $v$ such that $v\left(\Gamma_{\mathrm{k}}\right)=1$ but $v(\mathrm{Q})=0$; since $v\left(\Gamma_{\mathrm{k}}\right)=1$, by $\mathrm{VCL}, v(\mathrm{P} \supset \mathrm{Q})=1$ and $v(\mathrm{P})=1$; from the former, by TCL $(\supset), v(\mathrm{P})=0$ or $v(\mathrm{Q})=1$; so $v(\mathrm{Q})=1$. This is impossible; reject the assumption: $\Gamma_{\mathrm{K}} \models_{\mathrm{CL}} \mathrm{Q}$, which is to say, $\Gamma_{\mathrm{k}} \models_{\mathrm{CL}} A_{\mathrm{k}}$. 
$(\neg \mathrm{I})$ If $A_{\mathrm{k}}$ arises by $\neg \mathrm{I}$, then the picture is like this,

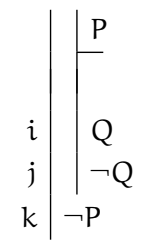

where $i, j<k$ and $A_{k}$ is $\neg$ P. By assumption, $\Gamma_{i} \models_{C L}$ Q and $\Gamma_{j} \models_{C L} \neg Q$; but by the nature of access, $\Gamma_{\mathrm{i}} \subseteq \Gamma_{\mathrm{k}} \cup\{\mathrm{P}\}$ and $\Gamma_{\mathrm{j}} \subseteq \Gamma_{\mathrm{k}} \cup\{\mathrm{P}\}$; so by LI.I, $\Gamma_{\mathrm{k}} \cup\{\mathrm{P}\} \models_{\mathrm{CL}} \mathrm{Q}$ and $\Gamma_{\mathrm{k}} \cup\{\mathrm{P}\} \models_{\mathrm{CL}} \neg \mathrm{Q}$. Suppose $\Gamma_{\mathrm{k}} \not \models_{\mathrm{CL}} \neg \mathrm{P}$; then by VCL, there is some $v$ such that $v\left(\Gamma_{\mathrm{k}}\right)=1$ but $v(\neg \mathrm{P})=0$; from the latter, by TCL $(\neg), v(\mathrm{P})=1$; so $v\left(\Gamma_{k}\right)=1$ and $v(\mathrm{P})=1$; so $v\left(\Gamma_{k} \cup\{\mathrm{P}\}\right)=1$; so by VCL, $v(\mathrm{Q})=1$ and $v(\neg \mathrm{Q})=1$; from the latter, by $\mathrm{TCL}(\neg), v(\mathrm{Q})=0$. This is impossible; reject the assumption: $\Gamma_{\mathrm{K}} \models_{\mathrm{CL}} \neg \mathrm{P}$, which is to say, $\Gamma_{\mathrm{k}} \models_{\mathrm{CL}} A_{\mathrm{k}}$.

$(\neg \mathrm{E})$

$(\vee I)$ If $A_{k}$ arises by $\vee I$, then the picture is like this,

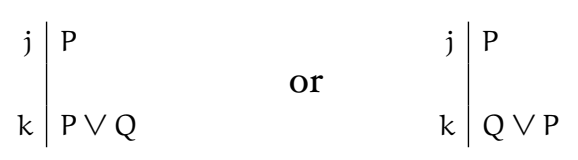

where $j<k$ and $A_{k}$ is $P \vee Q$ or $Q \vee P$. Consider the first case. By assumption, $\Gamma_{j} \models_{\mathrm{CL}}$ P; but by the nature of access, $\Gamma_{j} \subseteq \Gamma_{k}$; so by Lr.I, $\Gamma_{\mathrm{k}} \models_{\mathrm{CL}} \mathrm{P}$. Suppose $\Gamma_{\mathrm{k}} \forall_{\mathrm{CL}} \mathrm{P} \vee \mathrm{Q}$; then by VCL, there is some $v$ such that $v\left(\Gamma_{\mathrm{k}}\right)=1$ but $v(\mathrm{P} \vee \mathrm{Q})=0$; since $v\left(\Gamma_{\mathrm{k}}\right)=1$, by VCL, $v(\mathrm{P})=1$; but since $v(\mathrm{P} \vee \mathrm{Q})=0$, by TCL $(\vee), v(\mathrm{P})=0$ and $v(\mathrm{Q})=0$. This is impossible; reject the assumption: $\Gamma_{\mathrm{k}} \models_{\mathrm{CL}} \mathrm{P} \vee \mathrm{Q}$, which is to say, $\Gamma_{\mathrm{K}} \models_{\mathrm{CL}} A_{\mathrm{k}}$. And similarly when $A_{k}$ is $Q \vee P$.

$(\vee E)$ If $A_{k}$ arises by $\vee \mathrm{E}$, then the picture is like this,

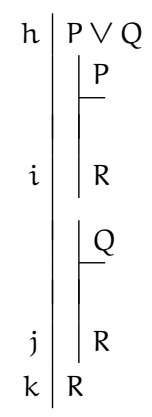

where $h, i, j<k$ and $A_{k}$ is $R$. By assumption, $\Gamma_{h} \models_{C L} P \vee Q, \Gamma_{i} \models_{C L} R$ and $\Gamma_{j} \models_{C L} R$; but by the nature of access, $\Gamma_{h} \subseteq \Gamma_{k}, \Gamma_{i} \subseteq \Gamma_{k} \cup\{P\}$ and $\Gamma_{\mathrm{j}} \subseteq \Gamma_{\mathrm{k}} \cup\{\mathrm{Q}\}$; so by LI.I, $\Gamma_{\mathrm{k}} \models_{\mathrm{CL}} \mathrm{P} \vee \mathrm{Q}, \Gamma_{\mathrm{k}} \cup\{\mathrm{P}\} \models_{\mathrm{CL}} \mathrm{R}$ and $\Gamma_{\mathrm{k}} \cup\{\mathrm{Q}\} \models_{\mathrm{CL}} \mathrm{R}$. Suppose $\Gamma_{k} \forall_{C L} R$; then by VCL, there is some $v$ such that $v\left(\Gamma_{k}\right)=1$ 
but $v(\mathrm{R})=0$. Since $v\left(\Gamma_{\mathrm{k}}\right)=1$, by VCL, $v(\mathrm{P} \vee \mathrm{Q})=1$; so by TCL $(\vee)$, $v(\mathrm{P})=1$ or $v(\mathrm{Q})=1$. Suppose, for the moment, that $v(\mathrm{P})=1$; then $v\left(\Gamma_{k}\right)=1$ and $v(\mathrm{P})=1$; so $v\left(\Gamma_{\mathrm{k}} \cup\{\mathrm{P}\}\right)=1$; so by VCL, $v(\mathrm{R})=1$; this is impossible; reject the assumption: $v(\mathrm{P}) \neq 1$; so $v(\mathrm{Q})=1$; so $v\left(\Gamma_{\mathrm{k}}\right)=1$ and $v(\mathrm{Q})=1$; so $v\left(\Gamma_{\mathrm{k}} \cup\{\mathrm{Q}\}\right)=1$; so by VCL, $v(\mathrm{R})=1$; this is impossible; reject the assumption: $\Gamma_{\mathrm{K}} \models_{\mathrm{CL}} \mathrm{R}$, which is to say, $\Gamma_{\mathrm{k}} \models_{\mathrm{CL}} A_{\mathrm{k}}$.

$(\equiv \mathrm{I})$

$(\equiv \mathrm{E})$

For any $i, \Gamma_{i} \models_{C L} A_{i}$.

THEOREM I.2 NCL is complete: if $\Gamma \models_{\mathrm{CL}}$ A then $\Gamma \vdash_{\mathrm{NCL}}$ A.

Con $\Gamma$ is COnsistent iff there is no $A$ such that $\Gamma \vdash_{\mathrm{NCL}} A$ and $\Gamma \vdash_{\mathrm{NCL}} \neg A$.

LI.2 If $\Gamma \nvdash_{\mathrm{NCL}} \neg \mathrm{P}$, then $\Gamma \cup\{\mathrm{P}\}$ is consistent.

Suppose $\Gamma H_{\text {NCL }} \neg P$ but $\Gamma \cup\{P\}$ is inconsistent. Then there is some $A$ such that $\Gamma \cup\{\mathrm{P}\} \vdash_{\mathrm{NCL}} A$ and $\Gamma \cup\{\mathrm{P}\} \vdash_{\mathrm{NCL}} \neg \mathrm{A}$. But then we can argue,

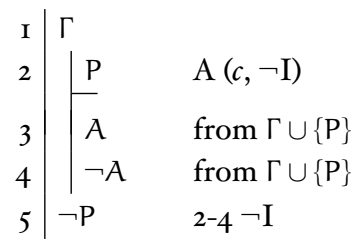

So $\Gamma \vdash_{\mathrm{NCL}} \neg \mathrm{P}$. But this is impossible; reject the assumption: if $\Gamma \nvdash_{\mathrm{NCL}} \neg \mathrm{P}$, then $\Gamma \cup\{\mathrm{P}\}$ is consistent.

LI.3 There is an enumeration of all the formulas, $A_{1}, A_{2} \ldots$

Proof by construction in the usual way. ${ }^{\mathrm{T}}$

Max $\Gamma$ is MAXIMAL iff for any $A$ either $\Gamma \vdash_{\mathrm{NCL}} A$ or $\Gamma \vdash_{\mathrm{NCL}} \neg A$.

$\mathrm{C}\left(\Gamma^{\prime}\right)$ We construct a $\Gamma^{\prime}$ from $\Gamma$ as follows. Set $\Omega_{0}=\Gamma$. By Li.3, there is an enumeration, $A_{1}, A_{2} \ldots$ of all the formulas; for any $A_{i}$ in this series set,

$$
\begin{array}{ll}
\Omega_{i}=\Omega_{i-1} & \text { if } \Omega_{i-1} \vdash_{\mathrm{NCL}} \neg A_{i} \\
\Omega_{i}=\Omega_{i-1} \cup\left\{A_{i}\right\} & \text { if } \Omega_{i-1} \nvdash_{\mathrm{NCL}} \neg A_{i}
\end{array}
$$

then

$$
\Gamma^{\prime}=\bigcup_{i \geqslant 0} \Omega_{i}
$$

${ }^{\mathrm{I}}$ For this, and extended discussion of the larger argument, see e.g. [I2, \$II.2]. 
LI.4 $\Gamma^{\prime}$ is maximal.

Suppose $\Gamma^{\prime}$ is not maximal. Then there is some $A_{i}$ such that $\Gamma^{\prime} H_{\mathrm{NCL}} A_{i}$ and $\Gamma^{\prime} H_{\mathrm{NCL}} \neg A_{i}$. Whatever $i$ may be, each member of $\Omega_{i-1}$ is in $\Gamma^{\prime}$; so if $\Omega_{i-1} \vdash_{\mathrm{NCL}} \neg A_{i}$ then $\Gamma^{\prime} \vdash_{\mathrm{NCL}} \neg A_{i}$; but $\Gamma^{\prime} \nvdash_{\mathrm{NCL}} \neg A_{i}$; so $\Omega_{i-1} \nvdash_{\mathrm{NCL}} \neg A_{i}$; so by construction, $\Omega_{i}=\Omega_{i-1} \cup\left\{A_{i}\right\}$; so by construction, $A_{i} \in \Gamma^{\prime}$; so $\Gamma^{\prime} \vdash_{\mathrm{NCL}} A_{i}$. This is impossible; reject the assumption: $\Gamma^{\prime}$ is maximal.

LI.5 If $\Gamma$ is consistent, then each $\Omega_{i}$ is consistent.

Suppose $\Gamma$ is consistent.

Basis: $\Omega_{0}=\Gamma$ and $\Gamma$ is consistent; so $\Omega_{0}$ is consistent.

Assp: For any $i, 0 \leqslant i<k, \Omega_{i}$ is consistent.

Show: $\Omega_{k}$ is consistent.

$\Omega_{k}$ is either $\Omega_{k-1}$ or $\Omega_{k-1} \cup\left\{A_{k}\right\}$. Suppose the former; by assumption, $\Omega_{k-1}$ is consistent; so $\Omega_{k}$ is consistent. Suppose the latter; then by construction, $\Omega_{\mathrm{k}-1} \nvdash_{\mathrm{NCL}} \neg A_{\mathrm{k}}$; so by LI.2, $\Omega_{k-1} \cup\left\{A_{k}\right\}$ is consistent; so $\Omega_{k}$ is consistent.

For any $i, \Omega_{i}$ is consistent.

LI.6 If $\Gamma$ is consistent, then $\Gamma^{\prime}$ is consistent.

Suppose $\Gamma$ is consistent, but $\Gamma^{\prime}$ is not; from the latter, there is some $\mathrm{P}$ such that $\Gamma^{\prime} \vdash_{\mathrm{NCL}} \mathrm{P}$ and $\Gamma^{\prime} \vdash_{\mathrm{NCL}} \neg \mathrm{P}$. Consider derivations DI and D2 of these results and the premises $A_{i} \ldots A_{j}$ of these derivations. Where $A_{j}$ is the last of these premises in the enumeration of formulas, by the construction of $\Gamma^{\prime}$, each of $A_{i} \ldots A_{j}$ must be a member of $\Omega_{j}$; so Di and $D_{2}$ are derivations from $\Omega_{j}$; so $\Omega_{j}$ is not consistent. But since $\Gamma$ is consistent, by $\mathrm{Lr}_{.5}, \Omega_{j}$ is consistent. This is impossible; reject the assumption: if $\Gamma$ is consistent then $\Gamma^{\prime}$ is consistent.

$\mathrm{C}(v)$ We construct a $C L$ interpretation $v$ based on $\Gamma^{\prime}$ as follows. For any parameter $p$, set $v(p)=1$ iff $\Gamma^{\prime} \vdash_{\text {NCL }} p$.

Li.7 If $\Gamma$ is consistent then for any $A, v(A)=1$ iff $\Gamma^{\prime} \vdash_{\mathrm{NCL}} A$.

Suppose $\Gamma$ is consistent. By Lr. $4, \Gamma^{\prime}$ is maximal; by Lr.6, $\Gamma^{\prime}$ is consistent. Now by induction on the number of operators in $A$,

Basis: If $A$ has no operators, then it is a parameter $p$ and by construction, $v(p)=1$ iff $\Gamma^{\prime} \vdash_{\mathrm{NCL}}$ p. So $v(A)=1$ iff $\Gamma^{\prime} \vdash_{\mathrm{NCL}} A$.

Assp: For any $i, 0 \leqslant i<k$, if $A$ has $i$ operators, then $v(A)=1$ iff $\Gamma^{\prime} \vdash_{\mathrm{NCL}} A$.

Show: If $A$ has $k$ operators, then $v(A)=1$ iff $\Gamma^{\prime} \vdash_{\mathrm{NCL}} A$.

If $A$ has $k$ operators, then it is of the form $\neg P, P \supset Q, P \wedge Q$, $\mathrm{P} \vee \mathrm{Q}$ or $\mathrm{P} \equiv \mathrm{Q}$ where $\mathrm{P}$ and $\mathrm{Q}$ have $<k$ operators. 
$(\neg) A$ is $\neg$ P. (i) Suppose $v(\mathrm{~A})=1$; then $v(\neg \mathrm{P})=1$; so by TCL $(\neg)$, $v(\mathrm{P})=0$; so by assumption, $\Gamma^{\prime} H_{\mathrm{NCL}} \mathrm{P}$; so by maximality, $\Gamma^{\prime} \vdash_{\mathrm{NCL}}$ $\neg \mathrm{P}$, where this is to say, $\Gamma^{\prime} \vdash_{\mathrm{NCL}} A$. (ii) Suppose $\Gamma^{\prime} \vdash_{\mathrm{NCL}} A$; then $\Gamma^{\prime} \vdash_{\mathrm{NCL}} \neg \mathrm{P}$; so by consistency, $\Gamma^{\prime} \nvdash_{\mathrm{NCL}} \mathrm{P}$; so by assumption, $v(\mathrm{P})=$ 0 ; so by TCL $(\neg), v(\neg P)=1$, where this is to say, $v(A)=1$. So $v(A)=1$ iff $\Gamma^{\prime} \vdash_{\text {NCL }} A$.

( $\supset$ ) $A$ is $P \supset$ Q. (i) Suppose $v(A)=1$ but $\Gamma^{\prime} H_{\mathrm{NCL}} A$; then $v(P \supset Q)=$ 1 but $\Gamma^{\prime} \nvdash_{\mathrm{NCL}} \mathrm{P} \supset \mathrm{Q}$. From the latter, by maximality, $\Gamma^{\prime} \vdash_{\mathrm{NCL}} \neg(\mathrm{P} \supset$ $\mathrm{Q})$; from this it follows, by simple derivations, that $\Gamma^{\prime} \vdash_{\mathrm{NCL}} \mathrm{P}$ and $\Gamma^{\prime} \vdash_{\mathrm{NCL}} \neg \mathrm{Q}$; so by consistency, $\Gamma^{\prime} \nvdash_{\mathrm{NCL}} \mathrm{Q}$; so by assumption, $v(\mathrm{P})=1$ and $v(\mathrm{Q})=0$; so by $\mathrm{TCL}(\supset), v(\mathrm{P} \supset \mathrm{Q})=0$. This is impossible; reject the assumption: if $v(A)=1$ then $\Gamma^{\prime} \vdash_{\mathrm{NCL}} A$.

(ii) Suppose $\Gamma^{\prime} \vdash_{\mathrm{NCL}}$ A but $v(A)=0$; then $\Gamma^{\prime} \vdash_{\mathrm{NCL}} \mathrm{P} \supset \mathrm{Q}$ but $v(\mathrm{P} \supset$ $\mathrm{Q})=0$. From the latter, by $\operatorname{TCL}(\supset), v(\mathrm{P})=1$ and $v(\mathrm{Q})=0$; so by assumption, $\Gamma^{\prime} \vdash_{\mathrm{NCL}} \mathrm{P}$ and $\Gamma^{\prime} \nvdash_{\mathrm{NCL}} \mathrm{Q}$; but since $\Gamma^{\prime} \vdash_{\mathrm{NCL}} \mathrm{P} \supset \mathrm{Q}$ and $\Gamma^{\prime} \vdash_{\mathrm{NCL}} \mathrm{P}$, by $(\supset \mathrm{E}), \Gamma^{\prime} \vdash_{\mathrm{NCL}} \mathrm{Q}$. This is impossible; reject the assumption: if $\Gamma^{\prime} \vdash_{\mathrm{NCL}} A$, then $v(A)=1$. So $v(A)=1$ iff $\Gamma^{\prime} \vdash_{\mathrm{NCL}} A$.

$(\wedge)$

$(\vee)$

$(\equiv)$

For any $A, v(A)=1$ iff $\Gamma^{\prime} \vdash_{\mathrm{NCL}} A$.

Lr.8 If $\Gamma$ is consistent, then $v(\Gamma)=1$.

Suppose $\Gamma$ is consistent and $A \in \Gamma$; then by construction, $A \in \Gamma^{\prime}$; so $\Gamma^{\prime} \vdash_{\mathrm{NCL}} A$; so since $\Gamma$ is consistent, by $\operatorname{Lr}_{.7}, v(A)=1$. And similarly for any $A \in \Gamma$. So $v(\Gamma)=1$.

Main result: Suppose $\Gamma \models_{\mathrm{CL}}$ A but $\Gamma \nvdash_{\mathrm{NCL}}$ A. By (DN), if $\Gamma \vdash_{\mathrm{NCL}} \neg \neg A$, then $\Gamma \vdash_{\mathrm{NCL}} A$; so $\Gamma \nvdash_{\mathrm{NCL}} \neg \neg A$; so by Lr.2, $\Gamma \cup\{\neg A\}$ is consistent; so by LI. 8, there is a $v$ constructed as above such that $v(\Gamma \cup\{\neg A\})=1$; so $v(\neg A)=1$; so by TCL $(\neg)$, $v(\mathrm{~A})=0$; so $v(\Gamma)=1$ and $v(\mathrm{~A})=0$; so by $\mathrm{VCL}, \Gamma \forall_{\mathrm{CL}} \mathrm{A}$. This is impossible; reject the assumption: if $\Gamma \models_{\mathrm{CL}} A$, then $\Gamma \vdash_{\mathrm{NCL}} A$.

2 NORMAL MODAL LOGICS: $K \alpha($ CH. 2,3)

\section{I LANGUAGE / SEMANTIC NOTIONS}

LK $\alpha$ The vocabulary consists of propositional parameters $p_{0}, p_{1} \ldots$ with the operators, $\neg, \wedge, \vee, \supset, \equiv, \square$ and $\diamond$. Each propositional parameter is a FORMULA; if $A$ and $B$ are formulas, so are $\neg A,(A \wedge B),(A \vee B)$, $(A \supset B),(A \equiv B), \square A$ and $\diamond A$. 
IK $\alpha$ For any of these systems except $\mathrm{Kv}$, an INTERPRETATION is a triple $\langle W, R, v\rangle$ where $W$ is a set of worlds, $R$ is a subset of $W^{2}=W \times W$, and $v$ is a function such that for any $w \in W$ and $p, v_{w}(p)=1$ or $v_{w}(p)=0$. For $x, y, z \in W$, where $\alpha$ is empty or indicates some combination of the following constraints,

$\eta \quad$ For any $x$, there is a $y$ such that $x R y$

extendability

$\rho$ for all $x, x R x$

$\sigma \quad$ for all $x, y$, if $x R y$ then $y R x$

$\tau \quad$ for all $x, y, z$, if $x$ Ry and $y R z$ then $x R z$ reflexivity symmetry transitivity

$\langle W, R, v\rangle$ is a $K \alpha$ interpretation when $R$ meets the constraints from $\alpha$. For $\mathrm{Kv}$, a model is just a pair $\langle\mathrm{W}, v\rangle$.

TK For complex expressions,

$(\neg) v_{w}(\neg A)=1$ if $v_{w}(A)=0$, and 0 otherwise.

$(\wedge) v_{w}(A \wedge B)=1$ if $v_{w}(A)=1$ and $v_{w}(B)=1$, and 0 otherwise.

(V) $v_{w}(A \vee B)=1$ if $v_{w}(A)=1$ or $v_{w}(B)=1$, and 0 otherwise.

( $\supset) v_{w}(\mathrm{~A} \supset \mathrm{B})=1$ if $v_{w}(\mathrm{~A})=0$ or $v_{w}(\mathrm{~B})=1$, and 0 otherwise.

$(\equiv) v_{w}(A \equiv B)=1$ if $v_{w}(A)=v_{w}(B)$, and 0 otherwise.

$(\diamond) v_{w}(\diamond A)=1$ if some $x \in W$ such that $w$ Rx has $v_{x}(A)=1$, and 0 otherwise.

( $\square) v_{w}(\square A)=1$ if all $x \in W$ such that $w$ Rx have $v_{x}(A)=1$, and 0 otherwise.

For $K v$, substitute for $(\diamond)$ and $(\square)$,

$(\diamond)_{\mathcal{v}} v_{w}(\diamond A)=1$ iff for some $x \in W, v_{x}(A)=1$.

$(\square)_{v} v_{w}(\square A)=1$ iff for all $x \in W, v_{x}(A)=1$.

For a set $\Gamma$ of formulas, $v_{w}(\Gamma)=1$ iff $v_{w}(A)=1$ for each $A \in \Gamma$; then,

$\mathrm{VK} \alpha \Gamma \models_{K \alpha} A$ iff there is no $\mathrm{K} \alpha$ interpretation $\langle\mathrm{W}, \mathrm{R}, v\rangle(\langle\mathrm{W}, v\rangle)$ and $w \in W$ such that $\nu_{w}(\Gamma)=1$ and $\nu_{w}(A)=0$.

\subsection{NATURAL DERIVATIONS: NK $\alpha$}

Where $s$ is any integer, let $A_{s}$ be a SUBSCRI PTED fORMula. For subscripts $s$ and $t$ allow also expressions of the sort, s.t. As in Priest, intuitively, subscripts indicate worlds, where $A_{s}$ is true or false at world $s$, and s.t just in case world $s$ has access to world t. Derivation rules apply to these expressions. Rules for $\neg$, $\wedge, \vee, \supset$, and $\equiv$ are like ones from before, but with consistent subscripts. Rules for $\square$ and $\diamond$ are new. ${ }^{2}$

\footnotetext{
${ }^{2}$ There is no uniformity about how to do natural deduction in modal logic. Most avoid subscripts altogether. Another option uses subscripts of the sort i.j ...k (cf. prefixes on tableaux in [2]); the result is elegant, but not so flexible as this account inspired by Priest, and we will need the flexibility, as we approach increasingly complex systems.
} 
$\mathrm{R} \mid \begin{aligned} & \mathrm{P}_{\mathrm{s}} \\ & \mathrm{P}_{\mathrm{s}}\end{aligned}$

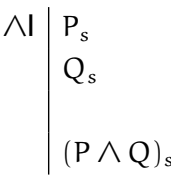

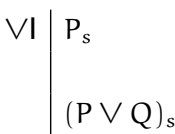

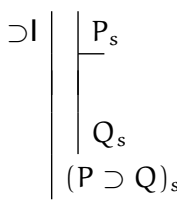

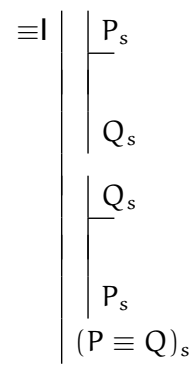



where $t$ does not appear in any undischarged premise or assumption

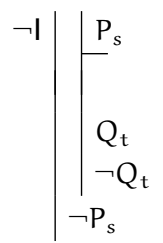

$\wedge \mathrm{E} \mid \begin{aligned} & (\mathrm{P} \wedge \mathrm{Q})_{s} \\ & \mathrm{P}_{\mathrm{s}}\end{aligned}$
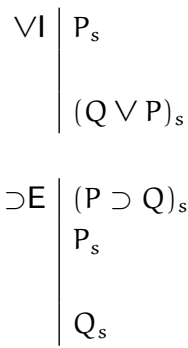

$\equiv \mathrm{E} \mid \begin{aligned} & (\mathrm{P} \equiv \mathrm{Q})_{\mathrm{s}} \\ & \mathrm{P}_{\mathrm{s}} \\ & \mathrm{Q}_{\mathrm{s}}\end{aligned}$

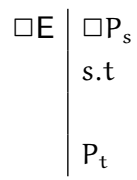

$\diamond \mathrm{I} \mid \begin{aligned} & \mathrm{P}_{\mathrm{t}} \\ & \text { s.t } \\ & \end{aligned}$

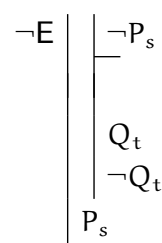

$\wedge \mathrm{E} \mid(\mathrm{P} \wedge \mathrm{Q})_{\mathrm{s}}$

$\mathrm{Q}_{\text {s }}$

$\vee \mathrm{E} \mid(\mathrm{P} \vee \mathrm{Q})_{s}$

$P_{s}$

$R_{\mathrm{t}}$

$\mathrm{Q}_{s}$

$R_{t}$

$R_{t}$

$\equiv \mathrm{E} \mid(\mathrm{P} \equiv \mathrm{Q})_{\mathrm{s}}$

$\mathrm{Q}_{s}$

$P_{s}$

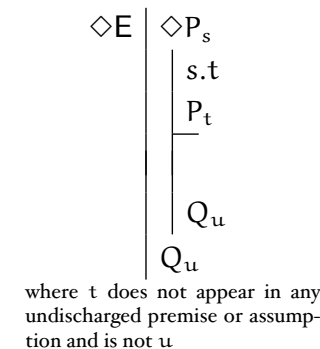

These are the rules of $N K$. Other systems $N K \alpha$ add from the following, for access manipulation, according to constraints in $\alpha$.
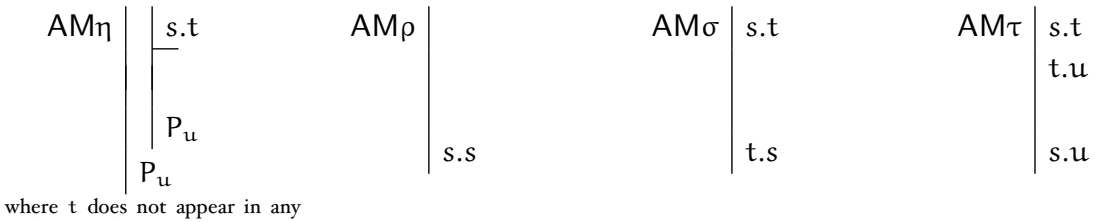

undischarged premise or assump-

tion and is not $u$

"Natural Derivations for Priest, An Introduction to Non-Classical Logic", Australasian fournal of Logic (5) 2006, 47-192 
$\mathrm{AM} \rho$ has no premise. For $N K v$, eliminate expressions of the sort s.t and rules for access manipulation. Let $T$ be an arbitrary tautology (say, $p \supset p$ ). Then for $\square \mathrm{I}, \square \mathrm{E}, \diamond \mathrm{I}$ and $\diamond \mathrm{E}$, substitute,
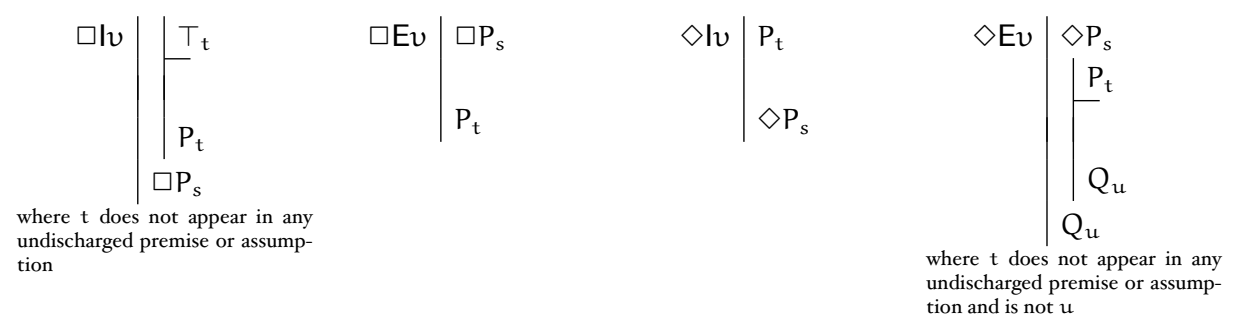

In these systems, every subscript is 0 , appears in a premise, or appears in the

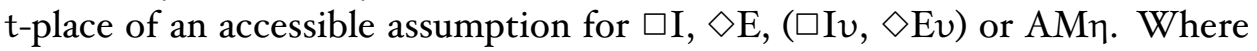
$\Gamma$ is a set of unsubscripted formulas, let $\Gamma_{0}$ be those same formulas, each with subscript 0 . Then,

$\mathrm{NK} \alpha \Gamma \vdash_{\mathrm{NK} \alpha} \mathrm{A}$ iff there is an $N K \alpha$ derivation of $A_{0}$ from the members of $\Gamma_{0}$.

Derived rules carry over from NCL as one would expect, with subscripts constant throughout. Thus, e.g.,

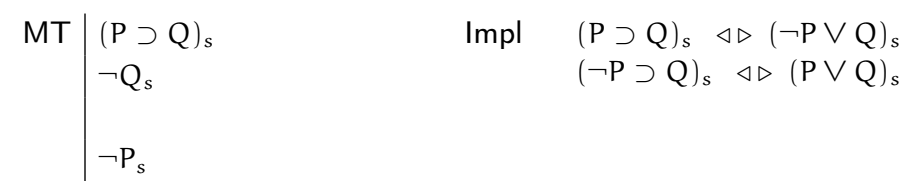

As examples, here are some derivations which exhibit left-hand forms of the following additional rule for modal negation,

$$
\begin{aligned}
& \text { MN } \quad \square P_{s} \quad \triangleleft \triangleright \neg \diamond \neg P_{s} \quad \neg \square P_{s} \quad \triangleleft \triangleright \diamond \neg P_{s} \\
& \diamond \mathrm{P}_{\mathrm{s}} \quad \triangleleft \triangleright \neg \square \neg \mathrm{P}_{\mathrm{s}} \quad \neg \diamond \mathrm{P}_{\mathrm{s}} \quad \triangleleft \triangleright \square \neg \mathrm{P}_{\mathrm{s}}
\end{aligned}
$$

\begin{tabular}{|c|c|c|}
\hline \\
\hline \multicolumn{3}{|c|}{$\begin{array}{l}\neg \diamond \neg \mathrm{P} \vdash_{\mathrm{NK}} \square \mathrm{P} \\
\mathrm{I} \mid \neg \diamond \neg \mathrm{P}_{0}\end{array}$} \\
\hline 2 & 0.1 & $\mathrm{~A}(\mathrm{~g}, \square \mathrm{I})$ \\
\hline 3 & $\neg \mathrm{P}_{1}$ & $\mathrm{~A}(c, \neg \mathrm{E})$ \\
\hline 4 & $\diamond \neg P_{0}$ & $2,3 \diamond \mathrm{I}$ \\
\hline 5 & $\neg \diamond \neg P_{0}$ & I $\mathrm{R}$ \\
\hline 6 & $P_{1}$ & $3-5 \neg \mathrm{E}$ \\
\hline 7 & $\square P_{0}$ & 2-6 $\square \mathrm{I}$ \\
\hline
\end{tabular}

as derived in $N K$ (and so any $N K \alpha$, excluding $N K v$, though this could be easily

\begin{tabular}{|c|c|c|}
\hline \multicolumn{3}{|c|}{$\square \mathrm{P} \vdash_{\mathrm{NK}} \neg \diamond \neg \mathrm{P}$} \\
\hline I & $\square \mathrm{P}_{0}$ & $\mathrm{P}$ \\
\hline 2 & $\diamond \neg P_{0}$ & $\mathrm{~A}(c, \neg \mathrm{I})$ \\
\hline 3 & 0.1 & $\mathrm{~A}(g, 2 \diamond \mathrm{E})$ \\
\hline 4 & $\neg \mathrm{P}_{1}$ & \\
\hline 5 & $\diamond \neg P_{0}$ & $\mathrm{~A}(c, \neg \mathrm{I})$ \\
\hline 6 & $\neg \mathrm{P}_{1}$ & ${ }_{4} \mathrm{R}$ \\
\hline 7 & $P_{1}$ & $\mathrm{I}, 3 \square \mathrm{E}$ \\
\hline 8 & $\neg \diamond \neg P_{0}$ & $5-7 \neg \mathrm{I}$ \\
\hline 9 & $\neg \diamond \neg \mathrm{P}_{0}$ & $2,3-8 \diamond \mathrm{E}$ \\
\hline IO & $\diamond \neg P_{0}$ & $2 \mathrm{R}$ \\
\hline II & $\neg \diamond \neg P_{0}$ & 2-IO $\neg \mathrm{I}$ \\
\hline
\end{tabular}
demonstrated as well). 


\begin{tabular}{|c|c|c|}
\hline \multicolumn{3}{|c|}{$\neg \square \neg \mathrm{P} \vdash_{\mathrm{NK}} \diamond \mathrm{P}$} \\
\hline I & $\neg \square \neg P_{0}$ & $\mathrm{P}$ \\
\hline 2 & $\neg \diamond \mathrm{P}_{0}$ & $\mathrm{~A}(c, \neg \mathrm{E})$ \\
\hline 3 & 0.1 & $\mathrm{~A}(\mathrm{~g}, \square \mathrm{I})$ \\
\hline 4 & $\mathrm{P}_{1}$ & $\mathrm{~A}(c, \neg \mathrm{I})$ \\
\hline 5 & $\diamond \mathrm{P}_{0}$ & $3,4 \diamond \mathrm{I}$ \\
\hline 6 & $\neg \diamond \mathrm{P}_{0}$ & $2 \mathrm{R}$ \\
\hline 7 & $\neg \mathrm{P}_{1}$ & $4^{-6} \neg \mathrm{I}$ \\
\hline 8 & $\square \neg P_{0}$ & 3-7 $\square \mathrm{I}$ \\
\hline 9 & $\neg \square \neg \mathrm{P}_{0}$ & I R \\
\hline IO & $\diamond P_{0}$ & $2-9 \neg E$ \\
\hline
\end{tabular}

\begin{tabular}{|c|c|c|}
\hline \multicolumn{3}{|c|}{$\diamond \mathrm{P} \vdash_{\mathrm{NK}} \neg \square \neg \mathrm{P}$} \\
\hline \multicolumn{3}{|c|}{$\begin{array}{l}\diamond \mathrm{P} \vdash_{\mathrm{NK}} \neg \square \neg \mathrm{P} \\
\mathrm{I} \mid \diamond \mathrm{P}_{0}\end{array}$} \\
\hline 2 & & $\mathrm{~A}(g, \diamond \mathrm{E})$ \\
\hline ; & \multicolumn{2}{|l|}{$P_{1}$} \\
\hline & $\square \neg \mathrm{P}_{0}$ & $\mathrm{~A}(c, \neg \mathrm{I})$ \\
\hline & $\neg \mathrm{P}_{1}$ & $2,4 \square \mathrm{E}$ \\
\hline & $P_{1}$ & $3 \mathrm{R}$ \\
\hline & $\neg \square \neg P_{0}$ & $4^{-6} \neg \mathrm{I}$ \\
\hline & $\neg \square \neg P_{0}$ & $\mathrm{I}, 2-7 \diamond \mathrm{E}$ \\
\hline
\end{tabular}

For examples in other systems, here are demonstrations of some characteristic principles:

\begin{tabular}{|c|c|c|}
\hline \multicolumn{3}{|c|}{$\vdash_{\mathrm{NK \eta}} \square \mathrm{P} \supset \diamond \mathrm{P}$} \\
\hline I & $\square \mathrm{P}_{0}$ & $\mathrm{~A}(g, \supset \mathrm{I})$ \\
\hline 2 & 0.1 & $\mathrm{~A}(g, \mathrm{AM} \eta)$ \\
\hline 3 & $P_{1}$ & $\mathrm{I}, 2 \square \mathrm{E}$ \\
\hline 4 & $\diamond \mathrm{P}_{0}$ & $2,3 \diamond \mathrm{I}$ \\
\hline 5 & $\diamond P_{0}$ & 2-4 $\mathrm{AM \eta}$ \\
\hline 6 & $(\square \mathrm{P} \supset \diamond \mathrm{P})_{0}$ & $\mathrm{I}-5 \supset \mathrm{I}$ \\
\hline \multicolumn{3}{|c|}{$\vdash_{\mathrm{NK \sigma} \sigma} \mathrm{P} \supset \square \diamond \mathrm{P}$} \\
\hline I & $P_{0}$ & $\mathrm{~A}(g, \supset \mathrm{I})$ \\
\hline 2 & 0.1 & $\mathrm{~A}(g, \square \mathrm{I})$ \\
\hline 3 & 1.0 & $2 \mathrm{AM} \sigma$ \\
\hline 4 & $\diamond \mathrm{P}_{1}$ & $\mathrm{I}, 3 \diamond \mathrm{I}$ \\
\hline 5 & $\square \diamond P_{0}$ & $2-4 \square \mathrm{I}$ \\
\hline 6 & $(\mathrm{P} \supset \square \diamond \mathrm{P})_{0}$ & $\mathrm{I}-5 \supset \mathrm{I}$ \\
\hline
\end{tabular}

$\vdash_{\mathrm{NK} \rho} \square \mathrm{P} \supset \mathrm{P}$
\begin{tabular}{l|ll}
$\mathrm{I}$ & $\square \mathrm{P}_{0}$ & $\mathrm{~A}(g, \supset \mathrm{I})$ \\
2 & $\mid 0.0$ & $\mathrm{AM} \rho$ \\
3 & $\mathrm{P}_{0}$ & $\mathrm{I}, 2 \square \mathrm{E}$ \\
4 & $(\square \mathrm{P} \supset \mathrm{P})_{0}$ & $\mathrm{I}-3 \supset \mathrm{I}$
\end{tabular}$$
\vdash_{\mathrm{NK \tau}} \square \mathrm{P} \supset \square \square \mathrm{P}
$$

\begin{tabular}{|c|c|c|}
\hline I & $\square \mathrm{P}_{0}$ & $\mathrm{~A}(g, \supset \mathrm{I})$ \\
\hline & 0.1 & $\mathrm{~A}(g, \square \mathrm{I})$ \\
\hline & 1.2 & $\mathrm{~A}(g, \square \mathrm{I})$ \\
\hline & 0.2 & $2,3 \mathrm{AM} \tau$ \\
\hline & $\mathrm{P}_{2}$ & $\mathrm{I}, 4 \square \mathrm{E}$ \\
\hline & $\square \mathrm{P}_{1}$ & 3-5 $\square \mathrm{I}$ \\
\hline & $\square \square P_{0}$ & 2-6 $\square \mathrm{I}$ \\
\hline 8 & $\square \mathrm{P} \supset \square \square \mathrm{P})_{0}$ & $\mathrm{I}-7 \supset \mathrm{I}$ \\
\hline
\end{tabular}




\begin{tabular}{|c|c|c|c|c|c|}
\hline \multicolumn{3}{|c|}{$\vdash_{N K \sigma \tau} \diamond P \supset \square \diamond P$} & \multicolumn{3}{|c|}{$\vdash_{\mathrm{NKv}} \diamond \mathrm{P} \supset \square \diamond \mathrm{P}$} \\
\hline I & $\diamond P_{0}$ & $\mathrm{~A}(g, \supset \mathrm{I})$ & I & $\diamond P_{0}$ & $\mathrm{~A}(g, \supset \mathrm{I})$ \\
\hline 2 & 0.1 & $\mathrm{~A}(g, \mathrm{I} \diamond \mathrm{E})$ & 2 & $\mathrm{P}_{1}$ & $\mathrm{~A}(g, \mathrm{I} \diamond \mathrm{E})$ \\
\hline 3 & $\mathrm{P}_{1}$ & & 3 & $\mid T_{2}$ & $\mathrm{~A}(g, \square \mathrm{I})$ \\
\hline 4 & 0.2 & $\mathrm{~A}(\mathrm{~g}, \square \mathrm{I})$ & 4 & $\diamond \mathrm{P}_{2}$ & $2 \diamond \mathrm{I}$ \\
\hline 5 & 2.0 & ${ }_{4} \mathrm{AM} \sigma$ & 5 & $\square \diamond P_{0}$ & 3-4 $\square \mathrm{I}$ \\
\hline 6 & 2.1 & $5,2 \mathrm{AM} \tau$ & 6 & $\square \diamond P_{0}$ & $\mathrm{I}, 2-5 \diamond \mathrm{E}$ \\
\hline 7 & $\diamond \mathrm{P}_{2}$ & $3,6 \diamond \mathrm{I}$ & 7 & $(\diamond P \supset \square \diamond P)_{0}$ & $\mathrm{I}-6 \supset \mathrm{I}$ \\
\hline 8 & $\square \diamond P_{0}$ & 4-7 $\square \mathrm{I}$ & & & \\
\hline 9 & $\square \diamond P_{0}$ & $\mathrm{I}, 2-8 \diamond \mathrm{E}$ & & & \\
\hline IO & $(\diamond \mathrm{P} \supset \square \diamond \mathrm{P})_{0}$ & $\mathrm{I}-9 \supset \mathrm{I}$ & & & \\
\hline
\end{tabular}

\subsection{SOUNDNESS AND COMPLETENESS}

Preliminaries (excluding $N K v$ ): Begin with generalized notions of validity. For a model $\langle W, R, v\rangle$, let $m$ be a map from subscripts into $W$. Say $\langle W, R, v\rangle_{m}$ is $\langle\mathrm{W}, \mathrm{R}, v\rangle$ with map $\mathrm{m}$. Then, where $\Gamma$ is a set of expressions of our language for derivations, $v_{m}(\Gamma)=1$ iff for each $A_{s} \in \Gamma, v_{m(s)}(A)=1$, and for each s.t $\in$ $\Gamma,\langle\mathrm{m}(\mathrm{s}), \mathrm{m}(\mathrm{t})\rangle \in \mathrm{R}$. Now expand notions of validity to include subscripted formulas, and alternate expressions as indicated in double brackets.

$\mathrm{VK} \alpha^{*} \Gamma \models_{K \alpha}^{*} A_{s} \llbracket$ s.t $\rrbracket$ iff there is no $K \alpha$ interpretation $\langle W, R, v\rangle_{m}$ such that $v_{\mathrm{m}}(\Gamma)=1$ but $v_{\mathrm{m}(\mathrm{s})}(\mathrm{A})=0 \llbracket\langle\mathrm{m}(\mathrm{s}), \mathrm{m}(\mathrm{t})\rangle \notin \mathrm{R} \rrbracket$.

$\mathrm{NK} \alpha^{*} \Gamma \vdash_{\mathrm{NK} \alpha}^{*} A_{s} \llbracket$ s.t $\rrbracket$ iff there is an $N K \alpha$ derivation of $A_{s} \llbracket$ s.t $\rrbracket$ from the members of $\Gamma$.

These notions reduce to the standard ones when all the members of $\Gamma$ and $A$ have subscript 0 (and so do not include expressions of the sort s.t). This is obvious for $\mathrm{NK} \alpha^{*}$. In the other case, there is a $\langle W, R, v\rangle_{m}$ that makes all the members of $\Gamma_{0}$ true and $A_{0}$ false just in case there is a world in $\langle W, R, v\rangle$ that makes the unsubscripted members of $\Gamma$ true and $A$ false. For the following, cases omitted are like ones worked, and so left to the reader.

THEOREM 2.I NK $\alpha$ is sound: If $\Gamma \vdash_{N K \alpha}$ A then $\Gamma \models_{K \alpha}$ A.

L2.I If $\Gamma \subseteq \Gamma^{\prime}$ and $\Gamma \models_{K \alpha}^{*} \mathrm{P}_{\mathrm{s}} \llbracket$ s.t $\rrbracket$, then $\Gamma^{\prime} \models_{K_{\alpha}}^{*} \mathrm{P}_{\mathrm{s}} \llbracket$ s.t $\rrbracket$.

Suppose $\Gamma \subseteq \Gamma^{\prime}$ and $\Gamma \models_{K_{\alpha} \alpha}^{*} P_{s} \llbracket$ s.t $\rrbracket$, but $\Gamma^{\prime} \nvdash_{K \alpha}^{*} P_{s} \llbracket$ s.t $\rrbracket$. From the latter, by $\mathrm{VK} \alpha^{*}$, there is some $K \alpha$ interpretation $\langle\mathrm{W}, \mathrm{R}, v\rangle_{\mathrm{m}}$ such that $v_{\mathrm{m}}\left(\Gamma^{\prime}\right)=1$ but $v_{\mathrm{m}(\mathrm{s})}(\mathrm{P})=0 \llbracket\langle\mathrm{m}(\mathrm{s}), \mathrm{m}(\mathrm{t})\rangle \notin \mathrm{R} \rrbracket$. But since $v_{\mathrm{m}}\left(\Gamma^{\prime}\right)=1$ and $\Gamma \subseteq \Gamma^{\prime}$, $v_{\mathfrak{m}}(\Gamma)=1$; so $v_{\mathfrak{m}}(\Gamma)=1$ but $v_{\mathrm{m}(\mathrm{s})}(\mathrm{P})=0 \llbracket\langle\mathrm{m}(\mathrm{s}), \mathrm{m}(\mathrm{t})\rangle \notin R \rrbracket$; so by $\mathrm{VK} \alpha^{*}, \Gamma \not \not_{K \alpha}^{*} P_{s} \llbracket s . t \rrbracket$. This is impossible; reject the assumption: if $\Gamma \subseteq \Gamma^{\prime}$ and $\Gamma \models_{K \alpha}^{*} P_{s} \llbracket$ s.t $\rrbracket$, then $\Gamma^{\prime} \models_{K \alpha}^{*} P_{s} \llbracket$ s.t $\rrbracket$. 
Main result: For each line in a derivation let $\mathcal{P}_{i}$ be the expression on line $i$ and $\Gamma_{i}$ be the set of all premises and assumptions whose scope includes line $i$. We set out to show "generalized" soundness: if $\Gamma \vdash_{N K \alpha}^{*} \mathcal{P}$ then $\Gamma \models_{K \alpha}^{*} \mathcal{P}$. As above, this reduces to the standard result when $\mathcal{P}$ and all the members of $\Gamma$ are formulas with subscript 0 . Suppose $\Gamma \vdash_{\mathrm{NK} \alpha}^{*} \mathcal{P}$. Then there is a derivation of $\mathcal{P}$ from premises in $\Gamma$ where $\mathcal{P}$ appears under the scope of the premises alone. By induction on line number of this derivation, we show that for each line $i$ of this derivation, $\Gamma_{i} \models_{K \alpha}^{*} \mathcal{P}_{i}$. The case when $\mathcal{P}_{i}=\mathcal{P}$ is the desired result.

Basis: $\mathcal{P}_{1}$ is a premise or an assumption $A_{s} \llbracket$ s.t $\rrbracket$. Then $\Gamma_{1}=\left\{A_{s}\right\} \llbracket\{$ s.t $\} \rrbracket$; so for any $\langle W, R, v\rangle_{m}, v_{m}\left(\Gamma_{1}\right)=1 \operatorname{iff} v_{m(s)}(A)=1 \llbracket\langle m(s), m(t)\rangle \in R \rrbracket$; so there is no $\langle\mathrm{W}, \mathrm{R}, v\rangle_{\mathrm{m}}$ such that $v_{\mathrm{m}}\left(\Gamma_{1}\right)=1$ but $v_{\mathrm{m}(\mathrm{s})}(\mathrm{A})=0 \llbracket\langle\mathrm{m}(\mathrm{s}), \mathrm{m}(\mathrm{t})\rangle \notin$ $R \rrbracket$. So by VK $\alpha^{*}, \Gamma_{1} \models_{K \alpha}^{*} A_{s} \llbracket$ s.t $\rrbracket$, where this is just to say, $\Gamma_{1} \models_{k \alpha}^{*} \mathcal{P}_{1}$.

Assp: For any $i, 1 \leqslant i<k, \Gamma_{i} \models_{k_{\alpha}}^{*} \mathcal{P}_{i}$.

Show: $\Gamma_{k} \models_{k \alpha}^{*} \mathcal{P}_{k}$.

$\mathcal{P}_{k}$ is either a premise, an assumption, or arises from previous lines by $\mathrm{R}$, $\supset \mathrm{I}, \supset \mathrm{E}, \wedge \mathrm{I}, \wedge \mathrm{E}, \neg \mathrm{I}, \neg \mathrm{E}, \vee \mathrm{I}, \vee \mathrm{E}, \equiv \mathrm{I}, \equiv \mathrm{E}, \square \mathrm{I}, \square \mathrm{E}, \diamond \mathrm{I}, \diamond \mathrm{E}$ or, depending on the system, $A M \eta, A M \rho, A M \sigma$ or $A M \tau$. If $\mathcal{P}_{k}$ is a premise or an assumption, then as in the basis, $\Gamma_{k} \models_{k \alpha}^{*} \mathcal{P}_{k}$. So suppose $\mathcal{P}_{k}$ arises by one of the rules.

$(\mathrm{R})$

$(\supset \mathrm{I})$

$(\supset \mathrm{E})$ If $\mathcal{P}_{k}$ arises by $\supset \mathrm{E}$, then the picture is like this,

\begin{tabular}{l|l}
$i$ & $(A \supset B)_{s}$ \\
$j$ & $A_{s}$ \\
$k$ & $B_{s}$
\end{tabular}

where $i, j<k$ and $\mathcal{P}_{k}$ is $B_{s}$. By assumption, $\Gamma_{i} \models_{k \alpha}^{*}(A \supset B)_{s}$ and $\Gamma_{j} \models_{K_{\alpha}}^{*} A_{s}$; but by the nature of access, $\Gamma_{\mathrm{i}} \subseteq \Gamma_{\mathrm{k}}$ and $\Gamma_{\mathrm{j}} \subseteq \Gamma_{\mathrm{k}}$; so by L2.I, $\Gamma_{k} \models_{k \alpha}^{*}(A \supset B)_{s}$ and $\Gamma_{k} \models_{k \alpha}^{*} A_{s}$. Suppose $\Gamma_{k} \not \nvdash_{k \alpha}^{*} B_{s}$; then by $V K \alpha^{*}$, there is some $K \alpha$ interpretation $\langle W, R, v\rangle_{m}$ such that $v_{m}\left(\Gamma_{k}\right)=1$ but $v_{\mathrm{m}(s)}(\mathrm{B})=0$; since $v_{\mathrm{m}}\left(\Gamma_{\mathrm{k}}\right)=1$, by $\mathrm{VK} \alpha^{*}, v_{\mathrm{m}(s)}(\mathrm{A} \supset \mathrm{B})=1$ and $v_{m(s)}(A)=1$; from the former, by $\operatorname{TK}(\supset), v_{m(s)}(A)=0$ or $v_{m(s)}(B)=$ 1; so $v_{m(s)}(B)=1$. This is impossible; reject the assumption: $\Gamma_{k} \models_{k \alpha}^{*} B_{s}$, which is to say, $\Gamma_{k} \models_{k \alpha}^{*} \mathcal{P}_{k}$.

$(\wedge \mathrm{I})$

$(\wedge \mathrm{E})$ 
$(\neg \mathrm{I})$ If $\mathcal{P}_{\mathrm{k}}$ arises by $\neg \mathrm{I}$, then the picture is like this,

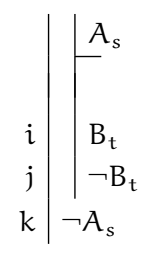

where $i, j<k$ and $\mathcal{P}_{k}$ is $\neg A_{s}$. By assumption, $\Gamma_{i} \models_{k \alpha}^{*} B_{t}$ and $\Gamma_{j} \models_{k \alpha}^{*}$ $\neg B_{t}$; but by the nature of access, $\Gamma_{i} \subseteq \Gamma_{k} \cup\left\{A_{s}\right\}$ and $\Gamma_{j} \subseteq \Gamma_{k} \cup\left\{A_{s}\right\}$; so by L2.I, $\Gamma_{k} \cup\left\{A_{s}\right\} \models_{k \alpha}^{*} B_{t}$ and $\Gamma_{k} \cup\left\{A_{s}\right\} \models_{k \alpha}^{*} \neg B_{t}$. Suppose $\Gamma_{k} \not \nvdash_{k \alpha}^{*}$ $\neg A_{s}$; then by $V K \alpha^{*}$, there is a $K \alpha$ interpretation $\langle W, R, v\rangle_{m}$ such that $v_{\mathfrak{m}}\left(\Gamma_{\mathrm{k}}\right)=1$ but $v_{\mathrm{m}(\mathrm{s})}(\neg \mathrm{A})=0$; so by TK $(\neg), v_{\mathrm{m}(s)}(\mathrm{A})=1$; so $v_{\mathrm{m}}\left(\Gamma_{\mathrm{k}}\right)=$ 1 and $v_{m(s)}(A)=1$; so $v_{m}\left(\Gamma_{k} \cup\left\{A_{s}\right\}\right)=1$; so by $V K \alpha^{*}, v_{m(t)}(B)=1$ and $v_{\mathrm{m}(\mathrm{t})}(\neg \mathrm{B})=1$; from the latter, by $\mathrm{TK}(\neg), v_{\mathrm{m}(\mathrm{t})}(\mathrm{B})=0$. This is impossible; reject the assumption: $\Gamma_{k} \models_{k \alpha}^{*} \neg A_{s}$, which is to say, $\Gamma_{k} \models_{k \alpha}^{*}$ $\mathcal{P}_{k}$.

$(\vee E)$ If $\mathcal{P}_{k}$ arises by $\vee E$, then the picture is like this,

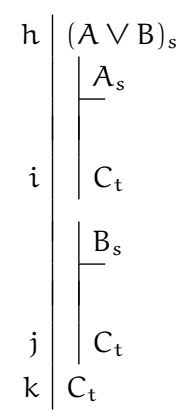

where $h, i, j<k$ and $\mathcal{P}_{k}$ is $C_{t}$. By assumption, $\Gamma_{h} \models_{k \alpha}^{*}(A \vee B)_{s}, \Gamma_{i} \models_{k \alpha}^{*} C_{t}$ and $\Gamma_{j} \models_{K_{\alpha}}^{*} C_{t}$; but by the nature of access, $\Gamma_{h} \subseteq \Gamma_{k}, \Gamma_{i} \subseteq \Gamma_{k} \cup\left\{A_{s}\right\}$ and $\Gamma_{j} \subseteq \Gamma_{k} \cup\left\{B_{s}\right\} ;$ so by L2.I, $\Gamma_{k} \models_{k_{\alpha}}^{*}(A \vee B)_{s}, \Gamma_{k} \cup\left\{A_{s}\right\} \models_{k \alpha}^{*} C_{t}$ and $\Gamma_{k} \cup\left\{B_{s}\right\} \models_{k \alpha}^{*} C_{t}$. Suppose $\Gamma_{k} \not \nvdash_{k \alpha}^{*} C_{t}$; then by $V K \alpha^{*}$, there is some $K \alpha$ interpretation $\langle W, R, v\rangle_{m}$ such that $v_{m}\left(\Gamma_{k}\right)=1$ but $v_{m(t)}(C)=0$. Since $v_{\mathfrak{m}}\left(\Gamma_{k}\right)=1$, by $V K \alpha^{*}, v_{m(s)}(A \vee B)=1$; so by $\mathrm{TK}(\vee), v_{m(s)}(A)=1$ or $v_{m(s)}(B)=1$. Suppose, for the moment, that $v_{m(s)}(A)=1$; then $v_{m}\left(\Gamma_{k}\right)=1$ and $v_{m(s)}(A)=1$; so $v_{m}\left(\Gamma_{k} \cup\left\{A_{s}\right\}\right)=1$; so by $V K \alpha^{*}$, $v_{\mathfrak{m}(\mathrm{t})}(\mathrm{C})=1$; this is impossible; reject the assumption: $v_{\mathrm{m}(\mathrm{s})}(\mathrm{A}) \neq 1$; so $v_{\mathfrak{m}(s)}(\mathrm{B})=1$; so $v_{\mathfrak{m}}\left(\Gamma_{k}\right)=1$ and $v_{\mathfrak{m}(s)}(\mathrm{B})=1$; so $v_{\mathfrak{m}}\left(\Gamma_{k} \cup\{\mathrm{B}\}\right)=1$; so by $\mathrm{VK} \alpha^{*}, v_{\mathrm{m}(\mathrm{t})}(\mathrm{C})=1$; this is impossible; reject the assumption: $\Gamma_{k} \models_{k \alpha}^{*} C_{t}$, which is to say, $\Gamma_{k} \models_{k \alpha}^{*} \mathcal{P}_{k}$.

$(\equiv \mathrm{I})$ 
( $\square$ I) If $\mathcal{P}_{k}$ arises by $\square \mathrm{I}$, then the picture is like this,<smiles>[Mg]C#[W]</smiles>

where $i<k$, $t$ does not appear in any member of $\Gamma_{k}$ (in any undischarged premise or assumption), and $\mathcal{P}_{k}$ is $\square A_{s}$. By assumption, $\Gamma_{i} \models_{k_{\alpha}}^{*} A_{t}$; but by the nature of access, $\Gamma_{i} \subseteq \Gamma_{k} \cup\{$ s.t $\}$; so by L2.I, $\Gamma_{k} \cup\{$ s.t $\} \models_{K_{\alpha}}^{*}$ $A_{t}$. Suppose $\Gamma_{k} \|_{K \alpha}^{*} \square A_{s}$; then by $V K \alpha^{*}$, there is a $K \alpha$ interpretation $\langle\mathrm{W}, \mathrm{R}, v\rangle_{\mathrm{m}}$ such that $v_{\mathrm{m}}\left(\Gamma_{\mathrm{k}}\right)=1$ but $v_{\mathrm{m}(\mathrm{s})}(\square \mathrm{A})=0$; so by $\mathrm{TK}(\square)$, there is some $w \in W$ such that $m(s) R w$ and $v_{w}(A)=0$. Now consider a map $\mathrm{m}^{\prime}$ like $\mathrm{m}$ except that $\mathrm{m}^{\prime}(\mathrm{t})=w$, and consider $\langle\mathrm{W}, \mathrm{R}, v\rangle_{\mathrm{m}^{\prime}}$; since $\mathrm{t}$ does not appear in $\Gamma_{k}$, it remains that $v_{m^{\prime}}\left(\Gamma_{k}\right)=1$; and since $m^{\prime}(t)=w$ and $\mathrm{m}^{\prime}(\mathrm{s})=\mathrm{m}(\mathrm{s}),\left\langle\mathrm{m}^{\prime}(\mathrm{s}), \mathrm{m}^{\prime}(\mathrm{t})\right\rangle \in \mathrm{R}$; so $v_{\mathrm{m}^{\prime}}\left(\Gamma_{\mathrm{k}} \cup\{\mathrm{s} . \mathrm{t}\}\right)=1$; so by $\mathrm{VK} \alpha^{*}$, $v_{m^{\prime}(t)}(A)=1$. But $m^{\prime}(t)=w$; so $v_{w}(A)=1$. This is impossible; reject the assumption: $\Gamma_{k} \models_{k \alpha}^{*} \square A_{s}$, which is to say, $\Gamma_{k} \models_{k \alpha}^{*} \mathcal{P}_{k}$.

( $\square \mathrm{E})$ If $\mathcal{P}_{k}$ arises by $\square \mathrm{E}$, then the picture is like this,

$$
\begin{array}{l|l}
i & \square A_{s} \\
j & s . t \\
k & A_{t}
\end{array}
$$

where $i, j<k$ and $\mathcal{P}_{k}$ is $A_{t}$. By assumption, $\Gamma_{i} \models_{k \alpha}^{*} \square A_{s}$ and $\Gamma_{j} \models_{k \alpha}^{*}$ s.t; but by the nature of access, $\Gamma_{\mathrm{i}} \subseteq \Gamma_{\mathrm{k}}$ and $\Gamma_{\mathrm{j}} \subseteq \Gamma_{\mathrm{k}}$; so by L2.I, $\Gamma_{\mathrm{k}} \models_{k_{\alpha}}^{*} \square A_{s}$ and $\Gamma_{k} \models_{k \alpha}^{*}$ s.t. Suppose $\Gamma_{k} \nvdash_{k \alpha}^{*} A_{t}$; then by $V K \alpha^{*}$, there is some $K \alpha$ interpretation $\langle W, R, v\rangle_{m}$ such that $v_{m}\left(\Gamma_{k}\right)=1$ but $v_{m(t)}(A)=0$; since $v_{\mathrm{m}}\left(\Gamma_{\mathrm{k}}\right)=1$, by $\mathrm{VK} \alpha^{*}, v_{\mathrm{m}(\mathrm{s})}(\square \mathrm{A})=1$ and $\langle\mathrm{m}(\mathrm{s}), \mathrm{m}(\mathrm{t})\rangle \in \mathrm{R}$; from the first of these, by $\operatorname{TK}(\square)$, any $w$ such that $m(s) R w$ has $v_{w}(A)=1$; so $v_{m(t)}(A)=1$. This is impossible; reject the assumption: $\Gamma_{k} \models_{k \alpha}^{*} A_{t}$, which is to say, $\Gamma_{k} \models_{k \alpha}^{*} \mathcal{P}_{k}$.

$(\diamond \mathrm{I})$

( $\diamond \mathrm{E})$ If $\mathcal{P}_{k}$ arises by $\diamond \mathrm{E}$, then the picture is like this,

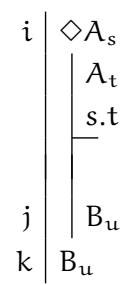


where $i, j<k$, $t$ does not appear in any member of $\Gamma_{k}$ (in any undischarged premise or assumption) and is not $u$, and $\mathcal{P}_{k}$ is $B_{u}$. By assumption, $\Gamma_{i} \models_{K_{\alpha}}^{*} \diamond A_{s}$ and $\Gamma_{j} \models_{K_{\alpha}}^{*} B_{u}$; but by the nature of access, $\Gamma_{i} \subseteq \Gamma_{k}$ and $\Gamma_{j} \subseteq \Gamma_{k} \cup\left\{A_{t}\right.$, s.t $\}$; so by L2.I, $\Gamma_{k} \models_{k \alpha}^{*} \diamond A_{s}$ and $\Gamma_{k} \cup\left\{A_{t}\right.$, s.t $\} \models_{k \alpha}^{*} B_{u}$. Suppose $\Gamma_{k} \not \nvdash_{k \alpha}^{*} B_{u}$; then by $V K \alpha^{*}$, there is a $K \alpha$ interpretation $\langle W, R, v\rangle_{m}$ such that $v_{\mathrm{m}}\left(\Gamma_{\mathrm{k}}\right)=1$ but $v_{\mathrm{m}(\mathfrak{u})}(\mathrm{B})=0$; since $v_{\mathrm{m}}\left(\Gamma_{\mathrm{k}}\right)=1$, by $\mathrm{VK} \alpha^{*}$, $v_{m(s)}(\diamond A)=1$; so by $\operatorname{TK}(\diamond)$, there is some $w \in W$ such that $m(s) R w$ and $v_{w}(\mathrm{~A})=1$. Now consider a map $\mathrm{m}^{\prime}$ like $\mathrm{m}$ except that $\mathrm{m}^{\prime}(\mathrm{t})=w$, and consider $\langle W, R, v\rangle_{m^{\prime}}$; since $t$ does not appear in $\Gamma_{k}$, it remains that $v_{m^{\prime}}\left(\Gamma_{k}\right)=1$; and since $m^{\prime}(s)=m(s)$ and $m^{\prime}(t)=w, v_{m^{\prime}(t)}(A)=1$ and $\left\langle\mathrm{m}^{\prime}(\mathrm{s}), \mathrm{m}^{\prime}(\mathrm{t})\right\rangle \in \mathrm{R}$; so $v_{\mathrm{m}^{\prime}}\left(\Gamma_{\mathrm{k}} \cup\left\{A_{\mathrm{t}}\right.\right.$, s.t $\left.\}\right)=1$; so by $\mathrm{VK} \alpha^{*}, v_{\mathrm{m}^{\prime}(\mathrm{u})}(\mathrm{B})=1$. But since $t \neq u, m^{\prime}(u)=m(u)$; so $v_{m(u)}(B)=1$. This is impossible; reject the assumption: $\Gamma_{k} \models_{k \alpha}^{*} B_{u}$, which is to say, $\Gamma_{k} \models_{k_{\alpha}}^{*} \mathcal{P}_{k}$.

$(\mathrm{AM \eta})$ If $\mathcal{P}_{k}$ arises by $\mathrm{AM \eta}$, then the picture is like this,

$$
\begin{array}{c|c}
i & \mid \\
k & A_{u} . t
\end{array}
$$

where $i<k$, $t$ does not appear in any member of $\Gamma_{k}$ (in any undischarged premise or assumption) and is not $u$, and $\mathcal{P}_{k}$ is $A_{u}$. Where this rule is included in $N K \alpha, K \alpha$ includes condition $\eta$. By assumption, $\Gamma_{i} \models_{K_{\alpha}}^{*} A_{u}$; but by the nature of access, $\Gamma_{i} \subseteq \Gamma_{k} \cup\{$ s.t $\}$; so by L2.I, $\Gamma_{k} \cup\{$ s.t $\} \models_{K \alpha}^{*}$ $A_{u}$. Suppose $\Gamma_{k} \not \not_{k \alpha}^{*} A_{u}$; then by $V K \alpha^{*}$, there is a $K \alpha$ interpretation $\langle W, R, v\rangle_{m}$ such that $v_{m}\left(\Gamma_{k}\right)=1$ but $v_{m(u)}(A)=0$. By condition $\eta$, there is a $w \in W$ such that $m(s) R w$; consider a map $m^{\prime}$ like $m$ except that $\mathrm{m}^{\prime}(\mathrm{t})=w$, and consider $\langle\mathrm{W}, \mathrm{R}, v\rangle_{\mathrm{m}^{\prime}}$; since $t$ does not appear in $\Gamma_{\mathrm{k}}$, it remains that $v_{m^{\prime}}\left(\Gamma_{k}\right)=1$; and since $m^{\prime}(s)=m(s)$ and $m^{\prime}(t)=w$, $\left\langle\mathrm{m}^{\prime}(\mathrm{s}), \mathrm{m}^{\prime}(\mathrm{t})\right\rangle \in \mathrm{R}$; so $v_{\mathrm{m}^{\prime}}\left(\Gamma_{\mathrm{k}} \cup\{\right.$ s.t $\left.\}\right)=1$; so by $\mathrm{VK} \alpha^{*}, v_{\mathrm{m}^{\prime}(u)}(A)=1$. But since $t \neq u, m^{\prime}(u)=m(u)$; so $v_{m(u)}(A)=1$. This is impossible; reject the assumption: $\Gamma_{k} \models_{k \alpha}^{*} A_{\mathfrak{u}}$, which is to say, $\Gamma_{k} \models_{k \alpha}^{*} \mathcal{P}_{k}$.

$(\mathrm{AM} \rho)$ If $\mathcal{P}_{k}$ arises by $\mathrm{AM} \rho$, then the picture is like this,<smiles>[AsH2]</smiles>

where $\mathcal{P}_{k}$ is s.s. Where this rule is in $N K \alpha, K \alpha$ includes condition $\rho$. Suppose $\Gamma_{\mathrm{k}} \not \nvdash_{k \alpha}^{*}$ s.s; then by $\mathrm{VK} \alpha^{*}$, there is some $\mathrm{K} \alpha$ interpretation $\langle\mathrm{W}, \mathrm{R}, v\rangle_{\mathrm{m}}$ such that $v_{\mathrm{m}}\left(\Gamma_{\mathrm{k}}\right)=1$ but $\langle\mathrm{m}(\mathrm{s}), \mathrm{m}(\mathrm{s})\rangle \notin \mathrm{R}$. But by condition $\rho$, for any $x \in W,\langle x, x\rangle \in R$; so $\langle m(s), m(s)\rangle \in R$. This is impossible; reject the assumption: $\Gamma_{k} \models_{k \alpha}^{*}$ s.s, which is to say, $\Gamma_{k} \models_{k \alpha}^{*} \mathcal{P}_{k}$.

$\left(\mathrm{AM \sigma} \sigma\right.$ If $\mathcal{P}_{k}$ arises by $\mathrm{AM} \sigma$, then the picture is like this, 


$$
\begin{array}{l|l}
i & s . t \\
k & \text { t.s }
\end{array}
$$

where $i<k$ and $\mathcal{P}_{k}$ is t.s. Where this rule is in $N K \alpha, K \alpha$ includes condition $\sigma$. By assumption, $\Gamma_{\mathrm{i}} \models_{K \alpha}^{*}$ s.t; but by the nature of access, $\Gamma_{i} \subseteq \Gamma_{k}$; so by L2.I, $\Gamma_{k} \models_{k \alpha}^{*}$ s.t. Suppose $\Gamma_{k} \nvdash_{k \alpha}^{*}$ t.s; then by VK $\alpha^{*}$, there is some $K \alpha$ interpretation $\langle W, R, v\rangle_{m}$ such that $v_{m}\left(\Gamma_{k}\right)=1$ but $\langle\mathrm{m}(\mathrm{t}), \mathrm{m}(\mathrm{s})\rangle \notin \mathrm{R}$; since $v_{\mathrm{m}}\left(\Gamma_{\mathrm{k}}\right)=1$, by $\mathrm{VK} \alpha^{*},\langle\mathrm{~m}(\mathrm{~s}), \mathrm{m}(\mathrm{t})\rangle \in \mathrm{R}$; and by condition $\sigma$, for any $\langle x, y\rangle \in R,\langle y, x\rangle \in R$; so $\langle m(t), m(s)\rangle \in R$. This is impossible; reject the assumption: $\Gamma_{k} \models_{k \alpha}^{*}$ t.s, which is to say, $\Gamma_{k} \models_{k \alpha}^{*} \mathcal{P}_{k}$.

$(\mathrm{AM} \tau)$ If $\mathcal{P}_{k}$ arises by $\mathrm{AM} \tau$, then the picture is like this,

$$
\begin{array}{c|c}
i & \text { s.t } \\
j & \text { t.u } \\
k & \text { s.u }
\end{array}
$$

where $i, j<k$ and $\mathcal{P}_{k}$ is s.u. Where this rule is in $N K \alpha, K \alpha$ includes condition $\tau$. By assumption, $\Gamma_{\mathrm{i}} \models_{k \alpha}^{*}$ s.t and $\Gamma_{j} \models_{k \alpha}^{*}$ t.u; but by the nature of access, $\Gamma_{i} \subseteq \Gamma_{k}$ and $\Gamma_{j} \subseteq \Gamma_{k}$; so by L2.I, $\Gamma_{k} \models_{k \alpha}^{*}$ s.t and $\Gamma_{k} \models_{k \alpha}^{*}$ t.u. Suppose $\Gamma_{k} \forall_{k \alpha}^{*}$ s.u; then by $V K \alpha^{*}$, there is some $K \alpha$ interpretation $\langle\mathrm{W}, \mathrm{R}, v\rangle_{\mathrm{m}}$ such that $v_{\mathrm{m}}\left(\Gamma_{\mathrm{k}}\right)=1$ but $\langle\mathrm{m}(\mathrm{s}), \mathrm{m}(\mathrm{u})\rangle \notin \mathrm{R}$; since $v_{\mathrm{m}}\left(\Gamma_{\mathrm{k}}\right)=$ 1, by $V K \alpha^{*},\langle\mathrm{~m}(\mathrm{~s}), \mathrm{m}(\mathrm{t})\rangle \in \mathrm{R}$ and $\langle\mathrm{m}(\mathrm{t}), \mathrm{m}(\mathrm{u})\rangle \in \mathrm{R}$; and by condition $\tau$, for any $\langle x, y\rangle,\langle y, z\rangle \in R,\langle x, z\rangle \in R$; so $\langle m(s), m(u)\rangle \in R$. This is impossible; reject the assumption: $\Gamma_{k} \models_{k_{\alpha}}^{*}$ s.u, which is to say, $\Gamma_{k} \models_{k \alpha}^{*} \mathcal{P}_{k}$.

For any $i, \Gamma_{i} \models_{k \alpha}^{*} \mathcal{P}_{i}$.

The argument for $N K v$ is similar (simpler) and so omitted.

THEOREM 2.2 NK $\alpha$ is complete: if $\Gamma \models_{\mathrm{K} \alpha}$ A then $\Gamma \vdash_{\mathrm{NK} \alpha}$ A.

Suppose $\Gamma \models_{K_{\alpha}} A$; then $\Gamma_{0} \models_{K \alpha}^{*} A_{0}$; we show that $\Gamma_{0} \vdash_{N K \alpha}^{*} A_{0}$. Again, this reduces to the standard notion. The method of our proof has advantages (especially for the quantificational case) over standard approaches to completeness for modal logic. Roughly, we construct a single set which is maximal and consistent relative to subscripted formulas, and use this to specify the model. The resultant proof is thus kept structurally parallel to the classical case. For the following, fix on some particular constraint(s) $\alpha$. Then definitions of consistency etc. are relative to it.

Con $\Gamma$ is CONSISTENT iff there is no $A_{s}$ such that $\Gamma \vdash_{\mathrm{NK} \alpha}^{*} A_{s}$ and $\Gamma \vdash_{\mathrm{NK} \alpha}^{*} \neg A_{s}$.

L2.2 If $s$ is 0 or appears in $\Gamma$, and $\Gamma H_{N K \alpha}^{*} \neg \mathrm{P}_{s}$, then $\Gamma \cup\left\{\mathrm{P}_{\mathrm{s}}\right\}$ is consistent. 
Suppose $s$ is 0 or appears in $\Gamma$ and $\Gamma H_{\mathrm{NK \alpha}}^{*} \neg \mathrm{P}_{\mathrm{s}}$ but $\Gamma \cup\left\{\mathrm{P}_{\mathrm{s}}\right\}$ is inconsistent. Then there is some $A_{t}$ such that $\Gamma \cup\left\{P_{s}\right\} \vdash_{\mathrm{NK} \alpha}^{*} A_{t}$ and $\Gamma \cup\left\{P_{s}\right\} \vdash_{\mathrm{NK} \alpha}^{*} \neg A_{t}$. But then we can argue,

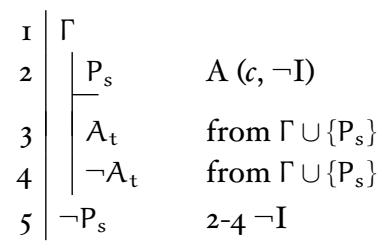

where the assumption is allowed insofar as $s$ is either 0 or appears in $\Gamma$; so $\Gamma \vdash_{\mathrm{NK} \alpha}^{*} \neg \mathrm{P}_{s}$. But this is impossible; reject the assumption: if $s$ is 0 or introduced in $\Gamma$ and $\Gamma \nvdash_{N K \alpha}^{*} \neg P_{s}$, then $\Gamma \cup\left\{P_{s}\right\}$ is consistent.

L2.3 There is an enumeration of all the subscripted formulas, $\mathcal{P}_{1} \mathcal{P}_{2} \ldots$

Proof by construction: Order non-subscripted formulas $A, B, C \ldots$ in the usual way. Then form a grid with formulas $A, B, C \ldots$ ordered across the top, and subscripts $1,2,3 \ldots$ down the side.

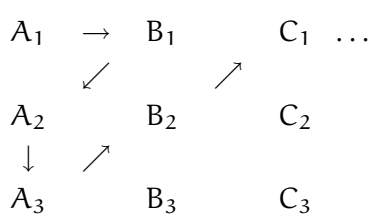

Order the members of the resultant grid, $A_{1}, B_{1}, A_{2} \ldots$ moving along the arrows from the upper left corner, down and to the right. ${ }^{3}$

Max $\Gamma$ is s-Maxi maL iff for any $A_{s}$ either $\Gamma \vdash_{\mathrm{NK} \alpha}^{*} A_{s}$ or $\Gamma \vdash_{\mathrm{NK} \alpha}^{*} \neg A_{s}$.

SGT $\Gamma$ is a sCAPEGOAT set iff for every formula of the form $\neg \square A_{s}$, if $\Gamma \vdash_{\mathrm{NK} \alpha}^{*}$ $\neg \square A_{s}$ then there is some $t$ such that $\Gamma \vdash_{\mathrm{NK} \alpha}^{*}$ s.t and $\Gamma \vdash_{\mathrm{NK} \alpha}^{*} \neg A_{\mathrm{t}}$.

$\mathrm{C}\left(\Gamma^{\prime}\right)$ For $\Gamma$ with unsubscripted formulas and the corresponding $\Gamma_{0}$, we construct $\Gamma^{\prime}$ as follows. Set $\Omega_{0}=\Gamma_{0}$. By L2.3, there is an enumeration, $\mathcal{P}_{1}, \mathcal{P}_{2} \ldots$ of all the subscripted formulas; let $\mathcal{E}_{0}$ be this enumeration. Then for the first $A_{s}$ in $\mathcal{E}_{i-1}$ such that $s$ is 0 or included in $\Omega_{i-1}$, let $\mathcal{E}_{i}$ be like $\varepsilon_{i-1}$ but without $A_{s}$, and set,

${ }^{3}$ As for rational numbers; see, e.g., [I2, \$2.I.I]. 


$$
\begin{array}{ll}
\Omega_{i}=\Omega_{i-1} & \text { if } \Omega_{i-1} \vdash_{N K \alpha}^{*} \neg A_{s} \\
\Omega_{i^{*}}=\Omega_{i-1} \cup\left\{A_{s}\right\} & \text { if } \Omega_{i-1} \nvdash_{N K \alpha}^{*} \neg A_{s}
\end{array}
$$

and

$$
\begin{array}{ll}
\Omega_{i}=\Omega_{i^{*}} & \text { if } A_{s} \text { is not of the form } \neg \square P_{s} \\
\Omega_{i}=\Omega_{i^{*}} \cup\left\{\text { s.t, } \neg P_{t}\right\} & \text { if } A_{s} \text { is of the form } \neg \square P_{s}
\end{array}
$$

-where $t$ is the first subscript not included in $\Omega_{i^{*}}$

then

$$
\Gamma^{\prime}=\bigcup_{i \geqslant 0} \Omega_{i}
$$

Note that there is always sure to be a subscript $t$ not in $\Omega_{i^{*}}$ insofar as there are infinitely many subscripts, and at any stage only finitely many formulas are added - the only subscripts in the initial $\Omega_{0}$ being 0 . Suppose $s$ is introduced in $\Gamma^{\prime}$; then there is some $\Omega_{i}$ in which it is first introduced; and any formula $\mathcal{P}_{j}$ in the original enumeration that has subscript $s$ is sure to be "considered" for inclusion at a subsequent stage.

L2.4 For any $s$ included in $\Gamma^{\prime}, \Gamma^{\prime}$ is s-maximal.

Suppose $s$ is included in $\Gamma^{\prime}$ but $\Gamma^{\prime}$ is not s-maximal. Then there is some $A_{s}$ such that $\Gamma^{\prime} \nvdash_{\mathrm{NK} \alpha}^{*} A_{s}$ and $\Gamma^{\prime} \nvdash_{\mathrm{NK} \alpha}^{*} \neg A_{s}$. For any $i$, each member of $\Omega_{i-1}$ is in $\Gamma^{\prime}$; so if $\Omega_{i-1} \vdash_{\mathrm{NK} \alpha}^{*} \neg A_{s}$ then $\Gamma^{\prime} \vdash_{\mathrm{NK} \alpha}^{*} \neg A_{s}$; but $\Gamma^{\prime} \forall_{\mathrm{NK} \alpha}^{*} \neg A_{s}$; so $\Omega_{i-1} \nvdash_{N K \alpha}^{*} \neg A_{s}$; so since $s$ is included in $\Gamma^{\prime}$, there is a stage in the construction that sets $\Omega_{i^{*}}=\Omega_{i-1} \cup\left\{A_{s}\right\}$; so by construction, $A_{s} \in \Gamma^{\prime}$; so $\Gamma^{\prime} \vdash_{\mathrm{NK} \alpha}^{*} A_{s}$. This is impossible; reject the assumption: $\Gamma^{\prime}$ is s-maximal.

L2.5 If $\Gamma_{0}$ is consistent, then each $\Omega_{i}$ is consistent.

Suppose $\Gamma_{0}$ is consistent.

Basis: $\Omega_{0}=\Gamma_{0}$ and $\Gamma_{0}$ is consistent; so $\Omega_{0}$ is consistent.

Assp: For any $i, 0 \leqslant i<k, \Omega_{i}$ is consistent.

Show: $\Omega_{\mathrm{k}}$ is consistent.

$\Omega_{k}$ is either (i) $\Omega_{k-1}$, or (ii) $\Omega_{k^{*}}=\Omega_{k-1} \cup\left\{A_{s}\right\}$ or (iii) $\Omega_{k^{*}} \cup$ $\left\{\right.$ s.t, $\left.\neg P_{\mathrm{t}}\right\}$.

(i) Suppose $\Omega_{k}$ is $\Omega_{k-1}$. By assumption, $\Omega_{k-1}$ is consistent; so $\Omega_{k}$ is consistent.

(ii) Suppose $\Omega_{k}$ is $\Omega_{k^{*}}=\Omega_{k-1} \cup\left\{A_{s}\right\}$. Then by construction, $s$ is 0 or in $\Omega_{k-1}$ and $\Omega_{k-1} \nvdash_{\mathrm{NK} \alpha}^{*} \neg A_{s}$; so by L2.2, $\Omega_{k-1} \cup\left\{A_{s}\right\}$ is consistent; so $\Omega_{k}$ is consistent.

(iii) Suppose $\Omega_{k}$ is $\Omega_{k^{*}} \cup\left\{\right.$ s.t, $\left.\neg P_{t}\right\}$. In this case, as above, $\Omega_{k *}$ is consistent and by construction, $\neg \square \mathrm{P}_{\mathrm{s}} \in \Omega_{\mathrm{k}^{*}}$. Suppose $\Omega_{\mathrm{k}}$ is inconsistent. Then there are $A_{\mathfrak{u}}$ and $\neg A_{\mathfrak{u}}$ such that $\Omega_{k^{*}} \cup$ $\left\{\right.$ s.t, $\left.\neg P_{\mathrm{t}}\right\} \vdash_{\mathrm{NK} \alpha}^{*} A_{\mathrm{u}}$ and $\Omega_{\mathrm{k}^{*}} \cup\left\{\right.$ s.t, $\left.\neg \mathrm{P}_{\mathrm{t}}\right\} \vdash_{\mathrm{NK} \alpha}^{*} \neg A_{\mathrm{u}}$. So reason as follows, 


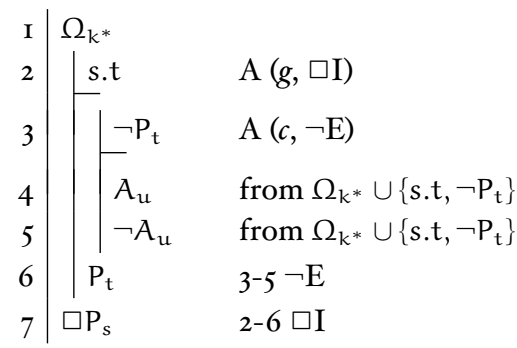

where, by construction, $t$ is not in $\Omega_{\mathrm{k}^{*}}$. So $\Omega_{\mathrm{k}^{*}} \vdash_{\mathrm{NK} \alpha}^{*} \square \mathrm{P}_{\mathrm{s}}$; but $\neg \square \mathrm{P}_{\mathrm{s}} \in \Omega_{\mathrm{k}^{*}}$; so $\Omega_{\mathrm{k}^{*}} \vdash_{\mathrm{NK} \alpha}^{*} \neg \square \mathrm{P}_{\mathrm{s}}$; so $\Omega_{\mathrm{k}^{*}}$ is inconsistent. This is impossible; reject the assumption: $\Omega_{k}$ is consistent.

For any $i, \Omega_{i}$ is consistent.

L2.6 If $\Gamma_{0}$ is consistent, then $\Gamma^{\prime}$ is consistent.

Suppose $\Gamma_{0}$ is consistent, but $\Gamma^{\prime}$ is not; from the latter, there is some $P_{s}$ such that $\Gamma^{\prime} \vdash_{N K \alpha}^{*} P_{s}$ and $\Gamma^{\prime} \vdash_{N K \alpha}^{*} \neg P_{s}$. Consider derivations Di and D2 of these results, and the premises $\mathcal{P}_{i} \ldots \mathcal{P}_{j}$ of these derivations. By construction, there is an $\Omega_{k}$ with each of these premises as a member; so Dr and $D_{2}$ are derivations from $\Omega_{k}$; so $\Omega_{k}$ is not consistent. But since $\Gamma_{0}$ is consistent, by L 2.5, $\Omega_{k}$ is consistent. This is impossible; reject the assumption: if $\Gamma_{0}$ is consistent then $\Gamma^{\prime}$ is consistent.

L2.7 If $\Gamma_{0}$ is consistent, then $\Gamma^{\prime}$ is a scapegoat set.

Suppose $\Gamma_{0}$ is consistent and $\Gamma^{\prime} \vdash_{\mathrm{NK} \alpha}^{*} \neg \square \mathrm{P}_{\mathrm{s}}$. By L2.6, $\Gamma^{\prime}$ is consistent; and by the constraints on subscripts, $s$ is included in $\Gamma^{\prime}$. Since $\Gamma^{\prime}$ is consistent, $\Gamma^{\prime} \nvdash_{\mathrm{NK} \alpha}^{*} \neg \neg \square \mathrm{P}_{s}$; so there is a stage in the construction process where $\Omega_{i^{*}}=\Omega_{i-1} \cup\left\{\neg \square P_{s}\right\}$ and $\Omega_{i}=\Omega_{i^{*}} \cup\left\{\right.$ s.t, $\left.\neg P_{t}\right\}$; so by construction, s.t $\in \Gamma^{\prime}$ and $\neg \mathrm{P}_{\mathrm{t}} \in \Gamma^{\prime}$; so $\Gamma^{\prime} \vdash_{\mathrm{NK \alpha}}^{*}$ s.t and $\Gamma^{\prime} \vdash_{\mathrm{NK} \alpha}^{*} \neg \mathrm{P}_{\mathrm{t}}$. So $\Gamma^{\prime}$ is a scapegoat set.

$\mathrm{C}$ (I) We construct an interpretation $\mathrm{I}=\langle\mathrm{W}, \mathrm{R}, v\rangle$ based on $\Gamma^{\prime}$ as follows. Let $W$ have a member $w_{s}$ corresponding to each subscript $s$ included in $\Gamma^{\prime}$. Then set $\left\langle w_{s}, w_{t}\right\rangle \in R$ iff $\Gamma^{\prime} \vdash_{\mathrm{NK} \alpha}^{*}$ s.t and $v_{w_{s}}(p)=1$ iff $\Gamma^{\prime} \vdash_{\mathrm{NK} \alpha}^{*} p_{s}$.

L2.8 If $\Gamma_{0}$ is consistent then for $\langle W, R, v\rangle$ constructed as above, and for any $s$ included in $\Gamma^{\prime}, v_{w_{\mathrm{s}}}(\mathrm{A})=1 \mathrm{iff} \Gamma^{\prime} \vdash_{\mathrm{NK} \alpha}^{*} A_{s}$.

Suppose $\Gamma_{0}$ is consistent and $s$ is included in $\Gamma^{\prime}$. By L2.4, $\Gamma^{\prime}$ is s-maximal. By L2.6 and L2.7, $\Gamma^{\prime}$ is consistent and a scapegoat set. Now by induction on the number of operators in $A_{s}$,

Basis: If $A_{s}$ has no operators, then it is a parameter $p_{s}$ and by construction, $v_{w_{s}}(p)=1$ iff $\Gamma^{\prime} \vdash_{\mathrm{NK} \alpha}^{*} p_{s}$. So $v_{w_{s}}(A)=1$ iff $\Gamma^{\prime} \vdash_{\mathrm{NK} \alpha}^{*} A_{s}$.

Assp: For any $i, 0 \leqslant i<k$, if $A_{s}$ has $i$ operators, then $v_{w_{s}}(A)=1$ iff $\Gamma^{\prime} \vdash_{\mathrm{NK} \alpha}^{*} A_{s}$. 
Show: If $A_{s}$ has $k$ operators, then $v_{w_{s}}(A)=1$ iff $\Gamma^{\prime} \vdash_{\mathrm{NK \alpha}}^{*} A_{s}$.

If $A_{s}$ has $k$ operators, then it is of the form $\neg P_{s},(P \supset Q)_{s}$, $(\mathrm{P} \wedge \mathrm{Q})_{s},(\mathrm{P} \vee \mathrm{Q})_{s},(\mathrm{P} \equiv \mathrm{Q})_{s}, \square \mathrm{P}_{\mathrm{s}}$ or $\diamond \mathrm{P}_{\mathrm{s}}$ where $\mathrm{P}$ and $\mathrm{Q}$ have $<\mathrm{k}$ operators.

$(\neg) A_{s}$ is $\neg P_{s}$. (i) Suppose $v_{w_{s}}(A)=1$; then $v_{w_{s}}(\neg P)=1$; so by $\mathrm{TK}(\neg), v_{w_{s}}(\mathrm{P})=0$; so by assumption, $\Gamma^{\prime} \nvdash_{\mathrm{NK \alpha}}^{*} \mathrm{P}_{s}$; so by $s-$ maximality, $\Gamma^{\prime} \vdash_{\mathrm{NK} \alpha}^{*} \neg P_{s}$, where this is to say, $\Gamma^{\prime} \vdash_{\mathrm{NK} \alpha}^{*} A_{s}$. (ii) Suppose $\Gamma^{\prime} \vdash_{\mathrm{NK} \alpha}^{*} A_{s}$; then $\Gamma^{\prime} \vdash_{\mathrm{NK \alpha}}^{*} \neg \mathrm{P}_{s}$; so by consistency, $\Gamma^{\prime} \vdash_{\mathrm{NK \alpha}}^{*} \mathrm{P}_{s}$; so by assumption, $v_{w_{s}}(\mathrm{P})=0$; so by $\mathrm{TK}(\neg), v_{w_{s}}(\neg \mathrm{P})=1$, where this is to say, $v_{w_{s}}(A)=1$. So $v_{w_{s}}(A)=1$ iff $\Gamma^{\prime} \vdash_{\mathrm{NK} \alpha}^{*} A_{s}$.

( $\supset A_{s}$ is $(P \supset Q)_{s}$. (i) Suppose $v_{w_{s}}(A)=1$ but $\Gamma^{\prime} \nvdash_{N K \alpha}^{*} A_{s}$; then $v_{w_{s}}(\mathrm{P} \supset \mathrm{Q})=1$ but $\Gamma^{\prime} \nvdash_{\mathrm{NK} \alpha}^{*}(\mathrm{P} \supset \mathrm{Q})_{s}$. From the latter, by $s-$ maximality, $\Gamma^{\prime} \vdash_{\mathrm{NK} \alpha}^{*} \neg(\mathrm{P} \supset \mathrm{Q})_{s}$; from this it follows, by simple derivations, that $\Gamma^{\prime} \vdash_{\mathrm{NK} \alpha}^{*} \mathrm{P}_{s}$ and $\Gamma^{\prime} \vdash_{\mathrm{NK} \alpha}^{*} \neg \mathrm{Q}_{s}$; so by consistency, $\Gamma^{\prime} \nvdash_{\mathrm{NK} \alpha}^{*} \mathrm{Q}_{s}$; so by assumption, $v_{w_{s}}(\mathrm{P})=1$ and $v_{w_{s}}(\mathrm{Q})=0$; so by $\mathrm{TK}(\supset), v_{w_{s}}(\mathrm{P} \supset \mathrm{Q})=0$. This is impossible; reject the assumption: if $v_{w_{s}}(A)=1$ then $\Gamma^{\prime} \vdash_{\mathrm{NK} \alpha}^{*} A_{s}$.

(ii) Suppose $\Gamma^{\prime} \vdash_{\mathrm{NK \alpha}}^{*} A_{s}$ but $v_{w_{s}}(A)=0$; then $\Gamma^{\prime} \vdash_{N K \alpha}^{*}(P \supset Q)_{s}$ but $v_{w_{s}}(\mathrm{P} \supset \mathrm{Q})=0$. From the latter, by $\mathrm{TK}(\supset), v_{w_{s}}(\mathrm{P})=1$ and $\nu_{w_{s}}(\mathrm{Q})=0$; so by assumption, $\Gamma^{\prime} \vdash_{\mathrm{NK} \alpha}^{*} \mathrm{P}_{\mathrm{s}}$ and $\Gamma^{\prime} \nvdash_{\mathrm{NK} \alpha}^{*} \mathrm{Q}_{s}$; but since $\Gamma^{\prime} \vdash_{\mathrm{NK} \alpha}^{*}(\mathrm{P} \supset \mathrm{Q})_{s}$ and $\Gamma^{\prime} \vdash_{\mathrm{NK} \alpha}^{*} \mathrm{P}_{\mathrm{s}}$, by $(\supset \mathrm{E}), \Gamma^{\prime} \vdash_{\mathrm{NK} \alpha}^{*} \mathrm{Q}_{s}$. This is impossible; reject the assumption: if $\Gamma^{\prime} \vdash_{\mathrm{NK} \alpha}^{*} A_{s}$, then $v_{w_{s}}(A)=1$. So $v_{w_{s}}(A)=1$ iff $\Gamma^{\prime} \vdash_{\mathrm{NK} \alpha}^{*} A_{s}$.

$(\wedge)$

$(\mathrm{V})$

$(\equiv)$

( $\square) A_{s}$ is $\square P_{s}$. (i) Suppose that $v_{w_{s}}(A)=1$ but $\Gamma^{\prime} \nvdash_{N K \alpha}^{*} A_{s}$; then $v_{w_{s}}(\square \mathrm{P})=1$ but $\Gamma^{\prime}{H_{N K \alpha}^{*}}^{*} \square \mathrm{P}_{s}$. From the latter, by s-maximality, $\Gamma^{\prime} \vdash_{\mathrm{NK \alpha}}^{*} \neg \square \mathrm{P}_{s}$; so, since $\Gamma^{\prime}$ is a scapegoat set, there is some $t$ such that $\Gamma^{\prime} \vdash_{\mathrm{NK} \alpha}^{*}$ s.t and $\Gamma^{\prime} \vdash_{\mathrm{NK} \alpha}^{*} \neg \mathrm{P}_{\mathrm{t}}$; from the first, by construction, $\left\langle w_{s}, w_{t}\right\rangle \in R$; and from the second, by consistency, $\Gamma^{\prime} \nvdash_{N K \alpha}^{*} P_{t}$; so by assumption, $v_{w_{t}}(\mathrm{P})=0$; but $w_{s} R w_{t}$; so by $\mathrm{TK}(\square), v_{w_{s}}(\square \mathrm{P})=0$. This is impossible; reject the assumption: if $v_{w_{s}}(A)=1$, then $\Gamma^{\prime} \vdash_{\mathrm{NK} \alpha}^{*} A_{s}$.

(ii) Suppose $\Gamma^{\prime} \vdash_{\mathrm{NK} \alpha}^{*} A_{s}$ but $v_{w_{s}}(A)=0$; then $\Gamma^{\prime} \vdash_{\mathrm{NK} \alpha}^{*} \square \mathrm{P}_{s}$ but $v_{w_{s}}(\square \mathrm{P})=0$. From the latter, by TK $(\square)$, there is some $w_{t} \in W$ such that $w_{s} R w_{t}$ and $v_{w_{t}}(\mathrm{P})=0$; so by assumption, $\Gamma^{\prime} H_{N K \alpha}^{*} P_{t}$; but since $w_{\mathrm{s}} \mathrm{R} w_{\mathrm{t}}$, by construction, $\Gamma^{\prime} \vdash_{\mathrm{NK} \alpha}^{*}$ s.t; so by $(\square \mathrm{E}), \Gamma^{\prime} \vdash_{\mathrm{NK} \alpha}^{*}$ $P_{t}$. This is impossible; reject the assumption: if $\Gamma^{\prime} \vdash_{N K \alpha} A_{s}$ then $v_{w_{s}}(A)=1$. So $v_{w_{s}}(A)=1$ iff $\Gamma^{\prime} \vdash_{N K \alpha}^{*} A_{s}$.

$(\diamond) A_{s}$ is $\diamond P_{s}$. (i) Suppose $v_{w_{s}}(A)=1$; then $v_{w_{s}}(\diamond P)=1$; so by $\mathrm{TK}(\diamond)$, there is some $w_{\mathrm{t}} \in \mathrm{W}$ such that $w_{\mathrm{s}} \mathrm{R} w_{\mathrm{t}}$ and $v_{w_{\mathrm{t}}}(\mathrm{P})=1$; 
so by assumption, $\Gamma^{\prime} \vdash_{N K \alpha}^{*} P_{t}$; but since $w_{s} R w_{t}$, by construction, $\Gamma^{\prime} \vdash_{\mathrm{NK} \alpha}^{*}$ s.t; so by $(\diamond \mathrm{I}), \Gamma^{\prime} \vdash_{\mathrm{NK} \alpha}^{*} \diamond \mathrm{P}_{s} ;$ so $\Gamma^{\prime} \vdash_{\mathrm{NK} \alpha}^{*} A_{s}$.

(ii)Suppose $\Gamma^{\prime} \vdash_{\mathrm{NK} \alpha}^{*} A_{s}$; then $\Gamma^{\prime} \vdash_{\mathrm{NK} \alpha}^{*} \diamond \mathrm{P}_{s}$; so by $(\mathrm{MN}), \Gamma^{\prime} \vdash_{\mathrm{NK} \alpha}^{*}$ $\neg \square \neg P_{s}$; so, since $\Gamma^{\prime}$ is a scapegoat set, there is some $t$ such that $\Gamma^{\prime} \vdash_{\mathrm{NK} \alpha}^{*}$ s.t and $\Gamma^{\prime} \vdash_{\mathrm{NK \alpha}}^{*} \neg \neg \mathrm{P}_{\mathrm{t}}$; so by $(\mathrm{DN}), \Gamma^{\prime} \vdash_{\mathrm{NK} \alpha}^{*} \mathrm{P}_{\mathrm{t}}$; so by assumption, $v_{w_{\mathrm{t}}}(\mathrm{P})=1$; but $\Gamma^{\prime} \vdash_{\mathrm{NK} \alpha}^{*}$ s.t; so by construction, $w_{s} R w_{t}$; so by $\operatorname{TK}(\diamond), v_{w_{s}}(\diamond \mathrm{P})=1$; so $v_{w_{s}}(A)=1$. So $v_{w_{s}}(A)=1$ iff $\Gamma^{\prime} \vdash_{\mathrm{NK} \alpha}^{*} A_{s}$.

For any $A_{s}, v_{w_{s}}(A)=1$ iff $\Gamma^{\prime} \vdash_{N K \alpha}^{*} A_{s}$.

L2.9 If $\Gamma_{0}$ is consistent, then $\langle W, R, v\rangle$ constructed as above is a $K \alpha$ interpretation.

In each case, we need to show that the interpretation meets the condition(s) $\alpha$. Suppose $\Gamma_{0}$ is consistent.

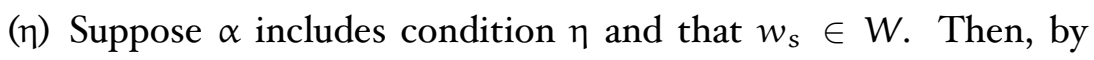
construction, $s$ is a subscript in $\Gamma^{\prime}$; so by reasoning as follows,

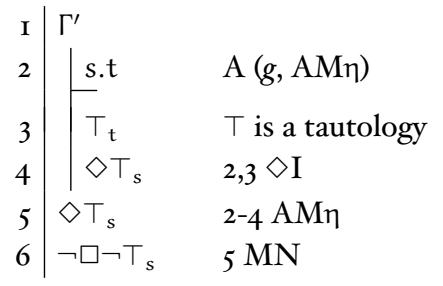

$\Gamma^{\prime} \vdash_{\mathrm{NK} \alpha}^{*} \neg \square \neg \top_{s}$; but by L2.7, $\Gamma^{\prime}$ is a scapegoat set; so there is a $t$ such that $\Gamma^{\prime} \vdash_{\mathrm{NK} \alpha}^{*}$ s.t; so by construction, $\left\langle w_{\mathrm{s}}, w_{\mathrm{t}}\right\rangle \in \mathrm{R}$ and $\eta$ is satisfied.

( $\rho$ ) Suppose $\alpha$ includes condition $\rho$ and $w_{s} \in W$. Then by construction, $s$ is a subscript in $\Gamma^{\prime}$; so by $(\mathrm{AM} \rho), \Gamma^{\prime} \vdash_{\mathrm{NK} \alpha}^{*}$ s.s; so by construction, $\left\langle w_{s}, w_{s}\right\rangle \in R$ and $\rho$ is satisfied.

( $\sigma)$ Suppose $\alpha$ includes condition $\sigma$ and $\left\langle w_{s}, w_{t}\right\rangle \in R$. Then by construction, $\Gamma^{\prime} \vdash_{\mathrm{NK} \alpha}^{*}$ s.t so by $(\mathrm{AM} \sigma), \Gamma^{\prime} \vdash_{\mathrm{NK} \alpha}^{*}$ t.s; so by construction, $\left\langle w_{\mathrm{t}}, w_{\mathrm{s}}\right\rangle \in \mathrm{R}$ and $\sigma$ is satisfied.

( $\tau$ ) Suppose $\alpha$ includes condition $\tau$ and $\left\langle w_{s}, w_{t}\right\rangle,\left\langle w_{t}, w_{\mathfrak{u}}\right\rangle \in R$. Then by construction, $\Gamma^{\prime} \vdash_{\mathrm{NK} \alpha}^{*}$ s.t and $\Gamma^{\prime} \vdash_{\mathrm{NK} \alpha}^{*}$ t.u; so by $(\mathrm{AM} \tau), \Gamma^{\prime} \vdash_{\mathrm{NK} \alpha}^{*}$ s.u; so by construction, $\left\langle w_{s}, w_{\mathfrak{u}}\right\rangle \in R$ and $\tau$ is satisfied.

MAP For any $w_{s} \in W$, set $m(s)=w_{s}$; otherwise $m(s)$ is arbitrary.

L2.IO If $\Gamma_{0}$ is consistent, then $v_{m}\left(\Gamma_{0}\right)=1$.

Suppose $\Gamma_{0}$ is consistent and $A_{0} \in \Gamma_{0}$; then by construction, $A_{0} \in \Gamma^{\prime}$; so $\Gamma^{\prime} \vdash_{\mathrm{NK} \alpha}^{*} A_{0}$; so since $\Gamma_{0}$ is consistent, by L2.8, $v_{w_{0}}(A)=1$. And similarly for any $A_{0} \in \Gamma_{0}$. But $m(0)=w_{0}$; so $v_{m}\left(\Gamma_{0}\right)=1$. 
Main result: Suppose $\Gamma \models_{K \alpha}$ A but $\Gamma \nvdash_{N K \alpha} A$. Then $\Gamma_{0} \models_{K \alpha}^{*} A_{0}$ but $\Gamma_{0} \nvdash_{N K \alpha}^{*} A_{0}$. By $(\mathrm{DN})$, if $\Gamma_{0} \vdash_{\mathrm{NK} \alpha}^{*} \neg \neg A_{0}$, then $\Gamma_{0} \vdash_{\mathrm{NK} \alpha}^{*} A_{0}$; so $\Gamma_{0} \nvdash_{\mathrm{NK} \alpha}^{*} \neg \neg A_{0}$; so by L 2.2, $\Gamma_{0} \cup\left\{\neg A_{0}\right\}$ is consistent; so by L2.9 and L2.Io, there is a $K \alpha$ interpretation $\langle W, R, v\rangle_{m}$ constructed as above such that $v_{\mathrm{m}}\left(\Gamma_{0} \cup\left\{\neg A_{0}\right\}\right)=1$; so $v_{\mathrm{m}(0)}(\neg A)=1$; so by $\mathrm{TK}(\neg), v_{\mathrm{m}(0)}(A)=0$; so $v_{\mathrm{m}}\left(\Gamma_{0}\right)=1$ and $v_{\mathrm{m}(0)}(A)=0$; so by $\mathrm{VK} \alpha^{*}, \Gamma_{0} \not \nvdash_{K \alpha}^{*} A_{0}$. This is impossible; reject the assumption: if $\Gamma \models_{K \alpha} A$, then $\Gamma \vdash_{\mathrm{NK} \alpha} A$.

The argument for $N K v$ is similar, and so omitted.

\section{NON-NORMAL MODAL LOGICS: $N \alpha(\mathrm{CH} .4)$}

\section{I LANGUAGE / SEMANTIC NOTIONS}

$\mathrm{LN} \alpha$ The basic language is the same as for $K \alpha$. The vOCABULARY consists of propositional parameters $p_{0}, p_{1} \ldots$ with the operators, $\neg, \wedge, \vee, \supset, \equiv$, $\square$ and $\diamond$. Each propositional parameter is a FORMULA; if $A$ and $B$ are formulas, so are $\neg A,(A \wedge B),(A \vee B),(A \supset B),(A \equiv B), \square A$ and $\diamond A$. In addition, we introduce $(A-3)$ as an abbreviation for $\square(A \supset B)$.

IN $\alpha$ An interpretation is $\langle W, N, R, v\rangle$ where $N \subseteq W . N$ is the set of normal worlds. Constraints on access are as for $\mathrm{K} \alpha$. Thus, where $\alpha$ is empty or indicates some combination of the following constraints,

$\begin{array}{lll}\eta & \text { For any } x, \text { there is a } y \text { such that } x R y & \text { extendability } \\ \rho & \text { for all } x, x R x & \text { reflexivity } \\ \sigma & \text { for all } x, y, \text { if } x R y \text { then } y R x & \text { symmetry } \\ \tau & \text { for all } x, y, z \text {, if } x R y \text { and } y R z \text { then } x R z & \text { transitivity }\end{array}$

$\langle W, N, R, v\rangle$ is an $N \alpha$ interpretation when $R$ meets the constraints from $\alpha$.

TN For complex expressions,

$(\neg) v_{w}(\neg A)=1$ if $v_{w}(A)=0$, and 0 otherwise.

$(\wedge) v_{w}(A \wedge B)=1$ if $v_{w}(A)=1$ and $v_{w}(B)=1$, and 0 otherwise.

(V) $v_{w}(\mathrm{~A} \vee \mathrm{B})=1$ if $v_{w}(\mathrm{~A})=1$ or $v_{w}(\mathrm{~B})=1$, and 0 otherwise.

(つ) $v_{w}(\mathrm{~A} \supset \mathrm{B})=1$ if $v_{w}(\mathrm{~A})=0$ or $v_{w}(\mathrm{~B})=1$, and 0 otherwise.

(三) $v_{w}(\mathrm{~A} \equiv \mathrm{B})=1$ if $v_{w}(\mathrm{~A})=v_{w}(\mathrm{~B})$, and 0 otherwise.

$(\diamond) v_{w}(\diamond A)=1$ if $w \notin N$ or some $x \in W$ such that $w R x$ has $v_{x}(A)=1$, and 0 otherwise.

( $\square) v_{w}(\square A)=1$ if $w \in N$ and all $x \in W$ such that $w \operatorname{Rx}$ have $v_{x}(A)=1$, and 0 otherwise.

For a set $\Gamma$ of formulas, $v_{w}(\Gamma)=1$ iff $v_{w}(A)=1$ for each $A \in \Gamma$; then,

$\mathrm{VN} \alpha \Gamma \models_{\mathrm{N}} A$ iff there is no $\mathrm{N} \alpha$ interpretation $\langle\mathrm{W}, \mathrm{N}, \mathrm{R}, v\rangle$ and $w \in \mathrm{N}$ such that $v_{w}(\Gamma)=1$ and $v_{w}(A)=0$. 


\subsection{NATURAL DERIVATIONS: $N N \alpha$}

All the rules are as in $N K \alpha$ except that whenever a subscript s.t is introduced for $\square \mathrm{I}$ or $\diamond \mathrm{E}$, either $s$ is 0 , or there is an additional premise of the sort $\square A_{s}$, or $\neg \diamond A_{s}$. The resulting change in constraints on these rules is small.


Derived rules carry over from $N K \alpha$. Note that $\mathrm{MN}$ remains as well. In addition, the following are derived rules for $-3 \mathrm{I}$ and $-3 \mathrm{E}$ in either $N K \alpha$ or $N N \alpha$.

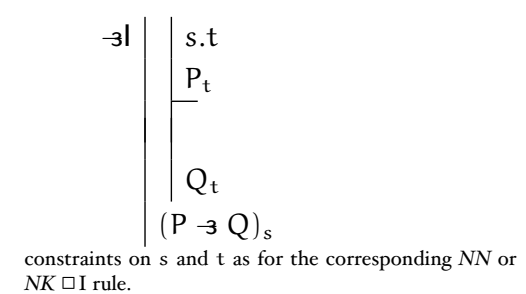

$$
{ }_{-3} \mathrm{E} \mid \begin{aligned}
& \left(\mathrm{P}-{ }_{3} \mathrm{Q}\right)_{s} \\
& \text { s.t } \\
& \mathrm{P}_{\mathrm{t}} \\
& \mathrm{Q}_{\mathrm{t}}
\end{aligned}
$$

NK $\square$ I rule.

We exhibit the new restrictions by considering derivations to show one part of $\mathrm{MN}$, that $\diamond \mathrm{P}_{\mathrm{s}} \vdash_{\mathrm{NN \alpha}} \neg \square \neg \mathrm{P}_{\mathrm{s}}$. In the case where $s \neq 0$, the derivation on the left violates the restriction on $\diamond \mathrm{E}$ in its last line.
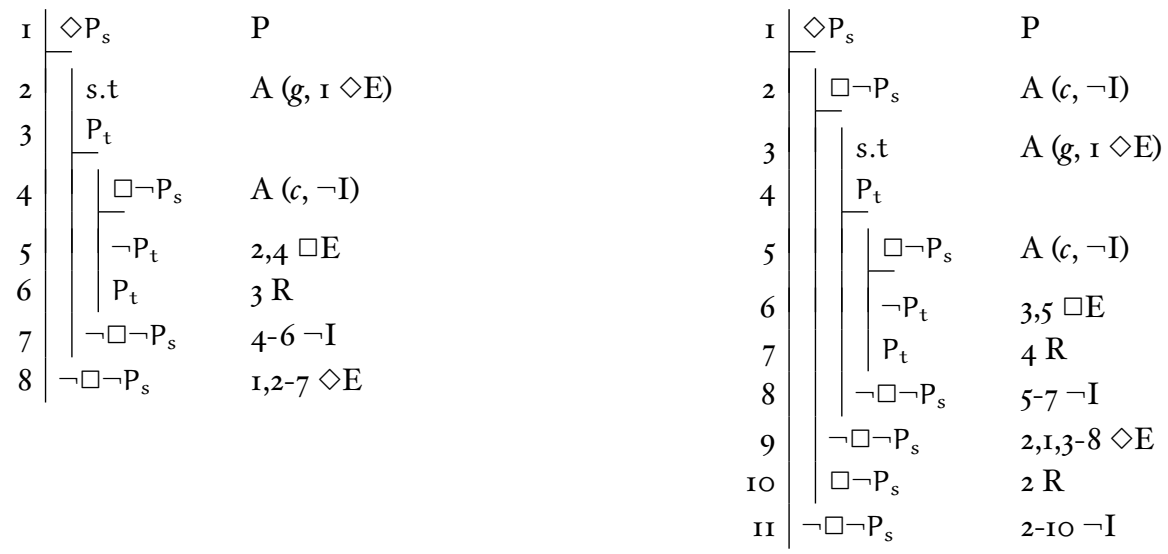

Supposing $s$ is 0 , each derivation is fine. However, if $s$ is other than 0 , on the left, (8) violates the restriction on $\diamond \mathrm{E}$, insofar as there is no accessible $\square \mathrm{P}_{\mathrm{s}}$ or $\neg \diamond \mathrm{P}_{\mathrm{s}}$. On the right, we get around the problem by making the assumption for $\neg \mathrm{I}$ prior to that for $\diamond \mathrm{E}$. Note that, in this case, we cite the line with $\square A_{s}$ for $\diamond \mathrm{E}$. Other derivations for $\mathrm{MN}$ go through as in the previous section. 


\section{$3 \cdot 3$ SOUNDNESS AND COMPLETENESS}

Preliminaries: Begin with generalized notions of validity. For a model $\langle W, N, R, v\rangle$, let $m$ be a map from subscripts into $W$ such that $m(0)$ is some member of $N$. Say $\langle W, N, R, v\rangle_{m}$ is $\langle W, N, R, v\rangle$ with map $m$. Then, where $\Gamma$ is a set of expressions of our language for derivations, $v_{\mathrm{m}}(\Gamma)=1$ iff for each $A_{\mathrm{s}} \in \Gamma$, $v_{\mathrm{m}(\mathrm{s})}(\mathrm{A})=1$, and for each $\mathrm{s.t} \in \Gamma,\langle\mathrm{m}(\mathrm{s}), \mathrm{m}(\mathrm{t})\rangle \in \mathrm{R}$. Now expand notions of validity to include subscripted formulas, and alternate expressions as indicated in double brackets.

$\mathrm{VN} \alpha^{*} \Gamma \models_{N \alpha}^{*} A_{s} \llbracket s . t \rrbracket$ iff there is no $\mathrm{N} \alpha$ interpretation $\langle W, N, R, v\rangle_{m}$ such that $v_{\mathrm{m}}(\Gamma)=1$ but $v_{\mathrm{m}(\mathrm{s})}(\mathrm{A})=0 \llbracket\langle\mathrm{m}(\mathrm{s}), \mathrm{m}(\mathrm{t})\rangle \notin \mathrm{R} \rrbracket$.

$\mathrm{NN} \alpha^{*} \Gamma \vdash_{\mathrm{NN} \alpha}^{*} A_{s} \llbracket$ s.t $\rrbracket$ iff there is an $N N \alpha$ derivation of $A_{s} \llbracket s . t \rrbracket$ from the members of $\Gamma$.

These notions reduce to the standard ones when all the members of $\Gamma$ and $A$ have subscript 0 (and so do not include expressions of the sort s.t). This is obvious for $\mathrm{NN} \alpha^{*}$. In the other case, there is a $\langle\mathrm{W}, \mathrm{N}, \mathrm{R}, v\rangle_{\mathrm{m}}$ and $w \in \mathrm{N}$ that makes all the members of $\Gamma_{0}$ true and $A_{0}$ false just in case there $i$ a world in $\mathrm{N}$ that makes the unsubscripted members of $\Gamma$ true and $A$ false. For the following, cases omitted are like ones worked, and so left to the reader.

THEOREM 3.I $N N \alpha$ is sound: If $\Gamma \vdash_{\mathrm{NN} \alpha}$ A then $\Gamma \models_{\mathrm{N} \alpha}$ A.

L3.I If $\Gamma \subseteq \Gamma^{\prime}$ and $\Gamma \models_{\mathrm{N} \alpha}^{*} \mathrm{P}_{\mathrm{s}} \llbracket$ s.t $\rrbracket$, then $\Gamma^{\prime} \models_{\mathrm{N} \alpha}^{*} \mathrm{P}_{\mathrm{s}} \llbracket$ s.t $\rrbracket$.

Reasoning parallel to that for L2.I of $N K \alpha$.

Main result: For each line in a derivation let $\mathcal{P}_{i}$ be the expression on line $i$ and $\Gamma_{i}$ be the set of all premises and assumptions whose scope includes line $i$. We set out to show "generalized" soundness: if $\Gamma \vdash_{\mathrm{NN \alpha}}^{*} \mathcal{P}$ then $\Gamma \models_{\mathrm{N \alpha}}^{*} \mathcal{P}$. Suppose $\Gamma \vdash_{\mathrm{NN} \alpha}^{*} \mathcal{P}$. Then there is a derivation of $\mathcal{P}$ from premises in $\Gamma$ where $\mathcal{P}$ appears under the scope of the premises alone. By induction on line number of this derivation, we show that for each line $i$ of this derivation, $\Gamma_{i} \models_{\mathrm{N} \alpha}^{*} \mathcal{P}_{i}$. The case when $\mathcal{P}_{i}=\mathcal{P}$ is the desired result.

Basis: $\mathcal{P}_{1}$ is a premise or an assumption $A_{s} \llbracket$ s.t $\rrbracket$. Then $\Gamma_{1}=\left\{A_{s}\right\} \llbracket\{$ s.t $\} \rrbracket$; so for any $\langle W, N, R, v\rangle_{m}, v_{m}\left(\Gamma_{1}\right)=1$ iff $v_{m(s)}(A)=1 \llbracket\langle m(s), m(t)\rangle \in R \rrbracket$; so there is no $\langle\mathrm{W}, \mathrm{N}, \mathrm{R}, v\rangle_{\mathrm{m}}$ such that $v_{\mathrm{m}}\left(\Gamma_{1}\right)=1$ but $v_{\mathrm{m}(\mathrm{s})}(\mathrm{A})=0$ $\llbracket\langle m(s), m(t)\rangle \notin R \rrbracket$. So by $V N \alpha^{*}, \Gamma_{1} \models_{N \alpha \alpha}^{*} A_{s} \llbracket s . t \rrbracket$, where this is just to say, $\Gamma_{1} \models_{\mathrm{N} \alpha}^{*} \mathcal{P}_{1}$.

Assp: For any $i, 1 \leqslant i<k, \Gamma_{i} \models_{N \alpha}^{*} \mathcal{P}_{i}$.

Shore: $\Gamma_{k} \models_{N \alpha}^{*} \mathcal{P}_{k}$. 
$\mathcal{P}_{k}$ is either a premise, an assumption, or arises from previous lines by $\mathrm{R}$, $\supset \mathrm{I}, \supset \mathrm{E}, \wedge \mathrm{I}, \wedge \mathrm{E}, \neg \mathrm{I}, \neg \mathrm{E}, \vee \mathrm{I}, \vee \mathrm{E}, \equiv \mathrm{I}, \equiv \mathrm{E}, \square \mathrm{I}, \neg \mathrm{E}, \diamond \mathrm{I}, \diamond \mathrm{E}$ or, depending on the system, $A M \eta, A M \rho, A M \sigma$ or $A M \tau$. If $\mathcal{P}_{k}$ is a premise or an assumption, then as in the basis, $\Gamma_{k} \models_{N \alpha}^{*} \mathcal{P}_{k}$. So suppose $\mathcal{P}_{k}$ arises by one of the rules.

(R)

$(\supset \mathrm{I})$

( $\mathrm{E})$ If $\mathcal{P}_{k}$ arises by $\supset \mathrm{E}$, then the picture is like this,

\begin{tabular}{l|l}
$i$ & $(A \supset B)_{s}$ \\
$j$ & $A_{s}$ \\
$k$ & $B_{s}$
\end{tabular}

where $i, j<k$ and $\mathcal{P}_{k}$ is $B_{s}$. By assumption, $\Gamma_{i} \models_{N^{\alpha}}^{*}(A \supset B)_{s}$ and $\Gamma_{\mathrm{j}} \models_{\mathrm{N} \alpha}^{*} A_{s}$; but by the nature of access, $\Gamma_{\mathrm{i}} \subseteq \Gamma_{\mathrm{k}}$ and $\Gamma_{\mathrm{j}} \subseteq \Gamma_{\mathrm{k}}$; so by L $\mathrm{L}_{3}$.I, $\Gamma_{k} \models_{N \alpha \alpha}^{*}(A \supset B)_{s}$ and $\Gamma_{k} \models_{N \alpha}^{*} A_{s}$. Suppose $\Gamma_{k} \not \nvdash_{N \alpha}^{*} B_{s}$; then by $V N \alpha^{*}$, there is some $N \alpha$ interpretation $\langle W, N, R, v\rangle_{m}$ such that $v_{m}\left(\Gamma_{k}\right)=1$ but $v_{\mathrm{m}(\mathrm{s})}(\mathrm{B})=0$; since $v_{\mathrm{m}}\left(\Gamma_{\mathrm{k}}\right)=1$, by $\mathrm{VN} \alpha^{*}, v_{\mathrm{m}(\mathrm{s})}(\mathrm{A} \supset \mathrm{B})=1$ and $v_{\mathfrak{m}(s)}(A)=1$; from the former, by $\mathrm{TN}(\supset), v_{\mathfrak{m}(s)}(A)=0$ or $v_{\mathfrak{m}(s)}(B)=$ 1; so $v_{m(s)}(B)=1$. This is impossible; reject the assumption: $\Gamma_{k} \models_{N \alpha}^{*} B_{s}$, which is to say, $\Gamma_{k} \models_{N \alpha}^{*} \mathcal{P}_{k}$.

$(\wedge \mathrm{I})$

$(\wedge \mathrm{E})$

$(\neg \mathrm{I})$ If $\mathcal{P}_{\mathrm{k}}$ arises by $\neg \mathrm{I}$, then the picture is like this,

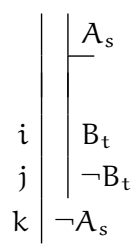

where $i, j<k$ and $\mathcal{P}_{k}$ is $\neg A_{s}$. By assumption, $\Gamma_{i} \models_{N_{\alpha}}^{*} B_{t}$ and $\Gamma_{j} \models_{N \alpha}^{*} \neg B_{t}$; but by the nature of access, $\Gamma_{i} \subseteq \Gamma_{k} \cup\left\{A_{s}\right\}$ and $\Gamma_{j} \subseteq \Gamma_{k} \cup\left\{A_{s}\right\}$; so by $\mathrm{L}_{3} . \mathrm{I}, \Gamma_{k} \cup\left\{A_{s}\right\} \models_{N^{\prime} \alpha}^{*} B_{t}$ and $\Gamma_{k} \cup\left\{A_{s}\right\} \models_{N \alpha}^{*} \neg B_{t}$. Suppose $\Gamma_{k} \not \nvdash_{N \alpha}^{*} \neg A_{s}$; then by $\mathrm{VN} \alpha^{*}$, there is an $\mathrm{N} \alpha$ interpretation $\langle W, N, R, v\rangle_{m}$ such that $v_{\mathrm{m}}\left(\Gamma_{\mathrm{k}}\right)=1$ but $v_{\mathrm{m}(\mathrm{s})}(\neg \mathrm{A})=0$; so by $\mathrm{TN}(\neg), v_{\mathrm{m}(\mathrm{s})}(\mathrm{A})=1$; so $v_{\mathrm{m}}\left(\Gamma_{\mathrm{k}}\right)=$ 1 and $v_{m(s)}(A)=1$; so $v_{m}\left(\Gamma_{k} \cup\left\{A_{s}\right\}\right)=1$; so by $V N \alpha^{*}, v_{m(t)}(B)=1$ and $v_{m(t)}(\neg \mathrm{B})=1$; from the latter, by $\mathrm{TN}(\neg), v_{m(t)}(\mathrm{B})=0$. This is impossible; reject the assumption: $\Gamma_{\mathrm{k}} \models_{\mathrm{N} \alpha}^{*} \neg A_{s}$, which is to say, $\Gamma_{\mathrm{k}} \models_{\mathrm{N}^{\prime}}^{*}$ $\mathcal{P}_{k}$.

$(\neg \mathrm{E})$ 
( $\square$ I) If $\mathcal{P}_{k}$ arises by $\square$ I, then the picture is like this,

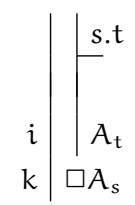

where $i<k$, s is 0 or introduced in some accessible $\square P_{s}$ or $\neg \diamond P_{s}, t$ does not appear in any member of $\Gamma_{k}$ (in any undischarged premise or assumption), and $\mathcal{P}_{k}$ is $\square A_{s}$. By assumption, $\Gamma_{i} \models_{N^{\prime \alpha}}^{*} A_{t}$; but by the nature of access, $\Gamma_{i} \subseteq \Gamma_{k} \cup\{$ s.t $\}$; so by L $3 . I, \Gamma_{k} \cup\{$ s.t $\} \models_{N_{\alpha}}^{*} A_{t}$. Suppose $\Gamma_{\mathrm{k}} \not \nvdash_{\mathrm{N} \alpha}^{*} \square A_{s}$; then by $\mathrm{VN} \alpha^{*}$, there is an $\mathrm{N} \alpha$ interpretation $\langle\mathrm{W}, \mathrm{N}, \mathrm{R}, v\rangle_{\mathrm{m}}$ such that $v_{m}\left(\Gamma_{k}\right)=1$ but $v_{m(s)}(\square A)=0$. If $s$ is 0 , then $m(s) \in N$; if $s$ is introduced in some $\square P_{s}$ on accessible line $j$, then by assumption, $\Gamma_{\mathrm{j}} \models_{\mathrm{N} \alpha}^{*} \square \mathrm{P}_{\mathrm{s}}$; but by the nature of access, $\Gamma_{\mathrm{j}} \subseteq \Gamma_{\mathrm{k}}$; so by $\mathrm{L}_{3} . \mathrm{I}, \Gamma_{\mathrm{k}} \models_{\mathrm{N} \alpha}^{*} \square \mathrm{P}_{s}$; so by $\mathrm{VN} \alpha^{*}, v_{\mathrm{m}(s)}(\square \mathrm{P})=1$; so by $\mathrm{TN}(\square), \mathrm{m}(\mathrm{s}) \in \mathrm{N}$; if $s$ is introduced in some $\neg \diamond \mathrm{P}_{\mathrm{s}}$ on an accessible line $\mathrm{j}$, then by assumption, $\Gamma_{\mathrm{j}} \models_{\mathrm{N} \alpha}^{*} \neg \diamond \mathrm{P}_{\mathrm{s}}$; but by the nature of access, $\Gamma_{j} \subseteq \Gamma_{k}$; so by L $3 . I, \Gamma_{k} \models_{N_{\alpha}}^{*} \neg \diamond P_{s}$; so by $\mathrm{VN} \alpha^{*}, v_{\mathrm{m}(s)}(\neg \diamond \mathrm{P})=1$; so by $\mathrm{TN}(\neg), v_{\mathrm{m}(s)}(\diamond \mathrm{P})=0$; so by $\mathrm{TN}(\diamond)$, $\mathrm{m}(s) \in \mathrm{N}$; in any case, then, $\mathrm{m}(s) \in \mathrm{N}$. So by $\mathrm{TN}(\square)$, there is some $w \in W$ such that $m(s) R w$ and $v_{w}(A)=0$. Now consider a map $m^{\prime}$ like $m$ except that $m^{\prime}(t)=w$, and consider $\langle W, N, R, v\rangle_{m^{\prime}}$; since $t$ does not appear in $\Gamma_{k}$, it remains that $v_{m^{\prime}}\left(\Gamma_{k}\right)=1$; and since $m^{\prime}(t)=w$ and $\mathrm{m}^{\prime}(\mathrm{s})=\mathrm{m}(\mathrm{s}),\left\langle\mathrm{m}^{\prime}(\mathrm{s}), \mathrm{m}^{\prime}(\mathrm{t})\right\rangle \in \mathrm{R}$; so $v_{\mathrm{m}^{\prime}}\left(\Gamma_{\mathrm{k}} \cup\{\mathrm{s} . \mathrm{t}\}\right)=1$; so by $\mathrm{VN} \alpha^{*}$, $v_{m^{\prime}(t)}(A)=1$. But $m^{\prime}(t)=w$; so $v_{w}(A)=1$. This is impossible; reject the assumption: $\Gamma_{k} \models_{N \alpha}^{*} \square A_{s}$, which is to say, $\Gamma_{k} \models_{N \alpha}^{*} \mathcal{P}_{k}$.

( $\square \mathrm{E})$ If $\mathcal{P}_{k}$ arises by $\square \mathrm{E}$, then the picture is like this,

\begin{tabular}{l|l}
$i$ & $\square A_{s}$ \\
$j$ & $s . t$ \\
$k$ & $A_{t}$
\end{tabular}

where $i, j<k$ and $\mathcal{P}_{k}$ is $A_{t}$. By assumption, $\Gamma_{i} \models_{N \alpha}^{*} \square A_{s}$ and $\Gamma_{j} \models_{N \alpha}^{*}$ s.t; but by the nature of access, $\Gamma_{\mathrm{i}} \subseteq \Gamma_{\mathrm{k}}$ and $\Gamma_{\mathrm{j}} \subseteq \Gamma_{\mathrm{k}}$; so by L3.I, $\Gamma_{\mathrm{k}} \models_{\mathrm{N}_{\alpha}}^{*} \square A_{s}$ and $\Gamma_{\mathrm{k}} \models_{\mathrm{N} \alpha}^{*}$ s.t. Suppose $\Gamma_{\mathrm{k}} \not \nvdash_{\mathrm{N} \alpha}^{*} A_{\mathrm{t}}$; then by $\mathrm{VN} \alpha^{*}$, there is some $\mathrm{N} \alpha$ interpretation $\langle\mathrm{W}, \mathrm{N}, \mathrm{R}, v\rangle_{\mathrm{m}}$ such that $\nu_{\mathrm{m}}\left(\Gamma_{\mathrm{k}}\right)=1$ but $v_{\mathrm{m}(\mathrm{t})}(\mathrm{A})=0$; since $v_{\mathrm{m}}\left(\Gamma_{\mathrm{k}}\right)=1$, by $\mathrm{VN} \alpha^{*}, v_{\mathrm{m}(\mathrm{s})}(\square \mathrm{A})=1$ and $\langle\mathrm{m}(\mathrm{s}), \mathrm{m}(\mathrm{t})\rangle \in \mathrm{R}$; from the first of these, by $\mathrm{TN}(\square)$, any $w$ such that $\mathrm{m}(\mathrm{s}) \mathrm{R} w$ has $v_{w}(A)=1$; 
so $v_{m(t)}(A)=1$. This is impossible; reject the assumption: $\Gamma_{k} \models_{N \alpha}^{*} A_{t}$, which is to say, $\Gamma_{k} \models_{N_{\alpha}}^{*} \mathcal{P}_{k}$.

$(\diamond \mathrm{I})$

$(\diamond \mathrm{E})$ If $\mathcal{P}_{\mathrm{k}}$ arises by $\diamond \mathrm{E}$, then the picture is like this,

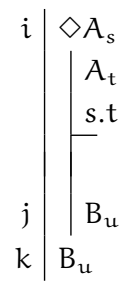

where $i, j<k, s$ is 0 or introduced in some accessible $\square P_{s}$ or $\neg \diamond P_{s}, t$ does not appear in any member of $\Gamma_{k}$ (in any undischarged premise or assumption) and is not $u$, and $\mathcal{P}_{k}$ is $B_{\mathfrak{u}}$. By assumption, $\Gamma_{i} \models_{N_{\alpha}}^{*} \diamond A_{s}$ and $\Gamma_{j} \models_{N \alpha}^{*} B_{u}$; but by the nature of access, $\Gamma_{i} \subseteq \Gamma_{k}$ and $\Gamma_{j} \subseteq \Gamma_{k} \cup\left\{A_{t}\right.$, s.t $\}$; so by $\mathrm{L}_{3} . \mathrm{I}, \Gamma_{\mathrm{k}} \models_{\mathrm{N} \alpha}^{*} \diamond A_{s}$ and $\Gamma_{k} \cup\left\{A_{\mathrm{t}}\right.$, s.t $\} \models_{\mathrm{N} \alpha}^{*} \mathrm{~B}_{\mathrm{u}}$. Suppose $\Gamma_{\mathrm{k}} \not \nvdash_{\mathrm{N} \alpha}^{*} B_{\mathfrak{u}}$; then by $\mathrm{VN} \alpha^{*}$, there is an $\mathrm{N} \alpha$ interpretation $\langle\mathrm{W}, \mathrm{N}, \mathrm{R}, v\rangle_{\mathrm{m}}$ such that $v_{\mathrm{m}}\left(\Gamma_{\mathrm{k}}\right)=1$ but $v_{\mathrm{m}(\mathrm{u})}(\mathrm{B})=0$. If $s$ is 0 , then $\mathrm{m}(\mathrm{s}) \in \mathrm{N}$; if $s$ is introduced in some $\square \mathrm{P}_{\mathrm{s}}$ on accessible line $h$, then by assumption, $\Gamma_{h} \models_{N_{\alpha}}^{*} \square \mathrm{P}_{s}$; but by the nature of access, $\Gamma_{\mathrm{h}} \subseteq \Gamma_{\mathrm{k}}$; so by $\mathrm{L}_{3} . \mathrm{I}, \Gamma_{\mathrm{k}} \models_{\mathrm{N \alpha}}^{*} \square \mathrm{P}_{s}$; so by $\mathrm{VN} \alpha^{*}, v_{\mathrm{m}(s)}(\square \mathrm{P})=1$; so by $\mathrm{TN}(\square), \mathrm{m}(\mathrm{s}) \in \mathrm{N}$; if $s$ is introduced in some $\neg \diamond P_{s}$ on an accessible line $h$, then by assumption, $\Gamma_{h} \models_{N \alpha}^{*} \neg \diamond P_{s}$; but by the nature of access, $\Gamma_{\mathrm{h}} \subseteq \Gamma_{\mathrm{k}}$; so by L $3 . \mathrm{I}, \Gamma_{\mathrm{k}} \models_{\mathrm{N} \alpha}^{*} \neg \diamond \mathrm{P}_{s}$; so by $\mathrm{VN} \alpha^{*}, v_{\mathrm{m}(s)}(\neg \diamond \mathrm{P})=1$; so by $\mathrm{TN}(\neg), v_{\mathrm{m}(s)}(\diamond \mathrm{P})=0$; so by $\mathrm{TN}(\diamond)$, $\mathrm{m}(\mathrm{s}) \in \mathrm{N}$; in any case, then, $\mathrm{m}(\mathrm{s}) \in \mathrm{N}$. Since $\nu_{\mathrm{m}}\left(\Gamma_{\mathrm{k}}\right)=1$, by $\mathrm{VN} \alpha^{*}$, $v_{\mathrm{m}(\mathrm{s})}(\diamond \mathrm{A})=1$; so by $\mathrm{TN}(\diamond)$, since $\mathrm{m}(\mathrm{s}) \in \mathrm{N}$, there is some $w \in \mathrm{W}$ such that $m(s) R w$ and $v_{w}(A)=1$. Now consider a map $m^{\prime}$ like $m$ except that $\mathrm{m}^{\prime}(\mathrm{t})=w$, and consider $\langle\mathrm{W}, \mathrm{N}, \mathrm{R}, v\rangle_{\mathrm{m}^{\prime}}$; since $\mathrm{t}$ does not appear in $\Gamma_{k}$, it remains that $v_{m^{\prime}}\left(\Gamma_{k}\right)=1$; and since $m^{\prime}(s)=m(s)$ and $m^{\prime}(t)=w$, $v_{m^{\prime}(t)}(A)=1$ and $\left\langle m^{\prime}(s), m^{\prime}(t)\right\rangle \in R$; so $v_{m^{\prime}}\left(\Gamma_{k} \cup\left\{A_{t}, s . t\right\}\right)=1$; so by $\mathrm{VN} \alpha^{*}, v_{\mathfrak{m}^{\prime}(\mathfrak{u})}(\mathrm{B})=1$. But since $t \neq u, \mathrm{~m}^{\prime}(\mathfrak{u})=\mathrm{m}(\mathfrak{u})$; so $v_{\mathfrak{m}(\mathfrak{u})}(\mathrm{B})=1$. This is impossible; reject the assumption: $\Gamma_{k} \models_{N \alpha}^{*} B_{\mathcal{u}}$, which is to say, $\Gamma_{\mathrm{k}} \models_{\mathrm{N} \alpha}^{*} \mathcal{P}_{\mathrm{k}}$.

$(\mathrm{AM \eta})$ If $\mathcal{P}_{k}$ arises by $\mathrm{AM \eta}$, then the picture is like this,

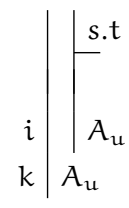

where $i<k$, $t$ does not appear in any member of $\Gamma_{k}$ (in any undischarged premise or assumption) and is not $u$, and $\mathcal{P}_{k}$ is $A_{u}$. Where this rule is included in $N N \alpha, N \alpha$ includes condition $\eta$. By assumption, $\Gamma_{i} \models_{N \alpha}^{*} A_{\mathcal{u}}$; 
but by the nature of access, $\Gamma_{\mathrm{i}} \subseteq \Gamma_{\mathrm{k}} \cup\{$ s.t $\}$; so by $\mathrm{L}_{3} . \mathrm{I}, \Gamma_{\mathrm{k}} \cup\{$ s.t $\} \models_{\mathrm{N}^{*}}^{*}$ $A_{u}$. Suppose $\Gamma_{k} \not \nvdash_{N \alpha}^{*} A_{u}$; then by $V N \alpha^{*}$, there is an $N \alpha$ interpretation $\langle W, N, R, v\rangle_{m}$ such that $v_{m}\left(\Gamma_{k}\right)=1$ but $v_{m(u)}(A)=0$. By condition $\eta$, there is a $w \in W$ such that $m(s) R w$; consider a map $m^{\prime}$ like $m$ except that $\mathrm{m}^{\prime}(\mathrm{t})=w$, and consider $\langle\mathrm{W}, \mathrm{N}, \mathrm{R}, v\rangle_{\mathrm{m}^{\prime}}$; since $\mathrm{t}$ does not appear in $\Gamma_{\mathrm{k}}$, it remains that $v_{\mathrm{m}^{\prime}}\left(\Gamma_{\mathrm{k}}\right)=1$; and since $\mathrm{m}^{\prime}(\mathrm{s})=\mathrm{m}(\mathrm{s})$ and $\mathrm{m}^{\prime}(\mathrm{t})=w$, $\left\langle\mathrm{m}^{\prime}(\mathrm{s}), \mathrm{m}^{\prime}(\mathrm{t})\right\rangle \in \mathrm{R}$; so $v_{\mathrm{m}^{\prime}}\left(\Gamma_{\mathrm{k}} \cup\{\right.$ s.t $\left.\}\right)=1$; so by $\mathrm{VN} \alpha^{*}, v_{\mathrm{m}^{\prime}(\mathrm{u})}(\mathrm{A})=1$. But since $t \neq u, m^{\prime}(u)=m(u)$; so $v_{\mathfrak{m}(\mathfrak{u})}(A)=1$. This is impossible; reject the assumption: $\Gamma_{k} \models_{N \alpha}^{*} A_{\mathfrak{u}}$, which is to say, $\Gamma_{k} \models_{N \alpha}^{*} \mathcal{P}_{k}$.

$(\mathrm{AM} \rho)$

$(\mathrm{AM \sigma})$

$(\mathrm{AM} \tau)$ If $\mathcal{P}_{\mathrm{k}}$ arises by $\mathrm{AM} \tau$, then the picture is like this,

$$
\begin{array}{l|l}
i & \text { s.t } \\
j & \text { t.u } \\
k & \text { s.u }
\end{array}
$$

where $i, j<k$ and $\mathcal{P}_{k}$ is s.u. Where this rule is in $N N \alpha, N \alpha$ includes condition $\tau$. By assumption, $\Gamma_{\mathrm{i}} \models_{\mathrm{N}_{\alpha}}^{*}$ s.t and $\Gamma_{\mathrm{j}} \models_{\mathrm{N} \alpha}^{*}$ t.u; but by the nature of access, $\Gamma_{i} \subseteq \Gamma_{k}$ and $\Gamma_{j} \subseteq \Gamma_{k}$; so by L $3 . I, \Gamma_{k} \models_{N \alpha \alpha}^{*}$ s.t and $\Gamma_{k} \models_{N \alpha}^{*}$ t.u. Suppose $\Gamma_{k} \not \nvdash_{N \alpha}^{*}$ s.u; then by $V N \alpha^{*}$, there is some $N \alpha$ interpretation $\langle\mathrm{W}, \mathrm{N}, \mathrm{R}, v\rangle_{\mathrm{m}}$ such that $v_{\mathrm{m}}\left(\Gamma_{\mathrm{k}}\right)=1$ but $\langle\mathrm{m}(\mathrm{s}), \mathrm{m}(\mathrm{u})\rangle \notin \mathrm{R}$; since $v_{\mathrm{m}}\left(\Gamma_{\mathrm{k}}\right)=1$, by VN $\alpha^{*},\langle\mathrm{~m}(\mathrm{~s}), \mathrm{m}(\mathrm{t})\rangle \in \mathrm{R}$ and $\langle\mathrm{m}(\mathrm{t}), \mathrm{m}(\mathrm{u})\rangle \in \mathrm{R}$; and by condition $\tau$, for any $\langle x, y\rangle,\langle y, z\rangle \in R,\langle x, z\rangle \in R$; so $\langle m(s), m(u)\rangle \in R$. This is impossible; reject the assumption: $\Gamma_{k} \models_{N \alpha \alpha}^{*}$ s.u, which is to say, $\Gamma_{\mathrm{k}} \models_{\mathrm{N} \alpha}^{*} \mathcal{P}_{\mathrm{k}}$.

For any $i, \Gamma_{i} \models_{N_{\alpha}}^{*} \mathcal{P}_{i}$.

THEOREM $3.2 N N \alpha$ is complete: if $\Gamma \models_{\mathrm{N}_{\alpha}}$ A then $\Gamma \vdash_{\mathrm{NN} \alpha}$ A.

Suppose $\Gamma \models_{N \alpha} A$; then $\Gamma_{0} \models_{N \alpha}^{*} A_{0}$; we show that $\Gamma_{0} \vdash_{N N \alpha}^{*} A_{0}$. Again, this reduces to the standard notion. For the following, fix on some particular constraint(s) $\alpha$. Then definitions of consistency etc. are relative to it.

Con $\Gamma$ is CONSISTENT iff there is no $A_{s}$ such that $\Gamma \vdash_{\mathrm{NN \alpha}}^{*} A_{s}$ and $\Gamma \vdash_{\mathrm{NN \alpha}}^{*} \neg A_{s}$.

$\mathrm{L}_{3.2}$ If $s$ is 0 or appears in $\Gamma$, and $\Gamma H_{\mathrm{NN} \alpha}^{*} \neg \mathrm{P}_{s}$, then $\Gamma \cup\left\{\mathrm{P}_{s}\right\}$ is consistent.

Suppose $s$ is 0 or appears in $\Gamma$ and $\Gamma H_{N N \alpha}^{*} \neg P_{s}$ but $\Gamma \cup\left\{P_{s}\right\}$ is inconsistent. Then there is some $A_{t}$ such that $\Gamma \cup\left\{P_{s}\right\} \vdash_{N N \alpha}^{*} A_{t}$ and $\Gamma \cup\left\{P_{s}\right\} \vdash_{N N \alpha}^{*} \neg A_{t}$. But then we can argue, 


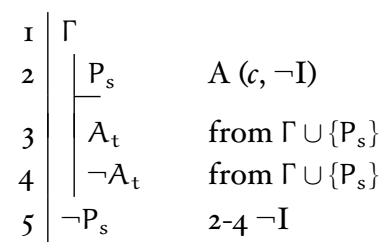

where the assumption is allowed insofar as $s$ is either 0 or appears in $\Gamma$; so $\Gamma \vdash_{\mathrm{NN} \alpha}^{*} \neg P_{s}$. But this is impossible; reject the assumption: if $s$ is 0 or introduced in $\Gamma$ and $\Gamma \mathrm{H}_{\mathrm{NN \alpha}}^{*} \neg \mathrm{P}_{s}$, then $\Gamma \cup\left\{\mathrm{P}_{s}\right\}$ is consistent.

$\mathrm{L}_{3.3}$ There is an enumeration of all the subscripted formulas, $\mathcal{P}_{1} \mathcal{P}_{2} \ldots$

Proof by construction as for $\mathrm{L} 2.3$ of $N K \alpha$.

Max $\Gamma$ is s-maximal iff for any $A_{s}$ either $\Gamma \vdash_{\mathrm{NN} \alpha}^{*} A_{s}$ or $\Gamma \vdash_{\mathrm{NN} \alpha}^{*} \neg A_{s}$.

SGT $\Gamma$ is a sCAPEgOAT set iff for every formula of the form $(\square P \wedge \neg \square A)_{s}$, if $\Gamma \vdash_{\mathrm{NN \alpha}}^{*}(\square \mathrm{P} \wedge \neg \square A)_{s}$ then there is some $t$ such that $\Gamma \vdash_{\mathrm{NN \alpha}}^{*}$ s.t and $\Gamma \vdash_{\mathrm{NN} \alpha}^{*} \neg A_{\mathrm{t}}$.

$\mathrm{C}\left(\Gamma^{\prime}\right)$ For $\Gamma$ with unsubscripted formulas and the corresponding $\Gamma_{0}$, we construct $\Gamma^{\prime}$ as follows. Set $\Omega_{0}=\Gamma_{0}$. By $\mathrm{L}_{3.3}$, there is an enumeration, $\mathcal{P}_{1}, \mathcal{P}_{2} \ldots$ of all the subscripted formulas; let $\mathcal{E}_{0}$ be this enumeration. Then for the first $A_{s}$ in $\mathcal{E}_{i-1}$ such that $s$ is 0 or included in $\Omega_{i-1}$, let $\mathcal{E}_{i}$ be like $\varepsilon_{i-1}$ but without $A_{s}$, and set,

$$
\begin{array}{ll}
\Omega_{i}=\Omega_{i-1} & \text { if } \Omega_{i-1} \vdash_{N N \alpha}^{*} \neg A_{s} \\
\Omega_{i^{*}}=\Omega_{i-1} \cup\left\{A_{s}\right\} & \text { if } \Omega_{i-1} \nvdash_{N N \alpha}^{*} \neg A_{s}
\end{array}
$$

and

$$
\begin{array}{ll}
\Omega_{i}=\Omega_{i^{*}} & \text { if } A_{s} \text { is not of the form }\left(\square Q \wedge \neg \square P_{s}\right) \\
\Omega_{i}=\Omega_{i^{*}} \cup\left\{\text { s.t, } \neg P_{t}\right\} & \text { if } A_{s} \text { is of the form }(\square Q \wedge \neg \square P)_{s}
\end{array}
$$$$
\text { -where } t \text { is the first subscript not included in } \Omega_{i^{*}}
$$

then

$$
\Gamma^{\prime}=\bigcup_{i \geqslant 0} \Omega_{i}
$$

Note that there is always sure to be a subscript $t$ not in $\Omega_{i^{*}}$ insofar as there are infinitely many subscripts, and at any stage only finitely many formulas are added - the only subscripts in the initial $\Omega_{0}$ being 0 . Suppose $s$ is introduced in $\Gamma^{\prime}$; then there is some $\Omega_{i}$ in which it is first introduced; and any formula $\mathcal{P}_{j}$ in the original enumeration that has subscript $s$ is sure to be "considered" for inclusion at a subsequent stage.

L3.4 For any s included in $\Gamma^{\prime}, \Gamma^{\prime}$ is s-maximal.

Suppose $s$ is included in $\Gamma^{\prime}$ but $\Gamma^{\prime}$ is not s-maximal. Then there is some $A_{s}$ such that $\Gamma^{\prime} \nvdash_{N N \alpha}^{*} A_{s}$ and $\Gamma^{\prime} \nvdash_{N N \alpha}^{*} \neg A_{s}$. For any $i$, each member of $\Omega_{i-1}$ is in $\Gamma^{\prime}$; so if $\Omega_{i-1} \vdash_{\mathrm{NN \alpha}}^{*} \neg A_{s}$ then $\Gamma^{\prime} \vdash_{\mathrm{NN \alpha}}^{*} \neg A_{s}$; but $\Gamma^{\prime} \nvdash_{\mathrm{NN} \alpha}^{*} \neg A_{s}$; so $\Omega_{i-1} \nvdash_{N N \alpha}^{*} \neg A_{s}$; so since $s$ is included in $\Gamma^{\prime}$, there is a stage in the 
construction that sets $\Omega_{\mathfrak{i}^{*}}=\Omega_{\mathfrak{i}-1} \cup\left\{A_{s}\right\}$; so by construction, $A_{s} \in \Gamma^{\prime}$; so $\Gamma^{\prime} \vdash_{\mathrm{NN} \alpha}^{*} A_{s}$. This is impossible; reject the assumption: $\Gamma^{\prime}$ is s-maximal.

$\mathrm{L}_{3} .5$ If $\Gamma_{0}$ is consistent, then each $\Omega_{i}$ is consistent.

Suppose $\Gamma_{0}$ is consistent.

Basis: $\Omega_{0}=\Gamma_{0}$ and $\Gamma_{0}$ is consistent; so $\Omega_{0}$ is consistent.

Assp: For any $i, 0 \leqslant i<k, \Omega_{i}$ is consistent.

Show: $\Omega_{k}$ is consistent.

$\Omega_{k}$ is either (i) $\Omega_{k-1}$, or (ii) $\Omega_{k^{*}}=\Omega_{k-1} \cup\left\{A_{s}\right\}$ or (iii) $\Omega_{k^{*}} \cup$ $\left\{\right.$ s.t, $\left.\neg P_{\mathrm{t}}\right\}$.

(i) Suppose $\Omega_{k}$ is $\Omega_{k-1}$. By assumption, $\Omega_{k-1}$ is consistent; so $\Omega_{k}$ is consistent.

(ii) Suppose $\Omega_{k}$ is $\Omega_{k^{*}}=\Omega_{k-1} \cup\left\{A_{s}\right\}$. Then by construction, $s$ is 0 or in $\Omega_{k-1}$ and $\Omega_{k-1} \nvdash_{\mathrm{NN} \alpha}^{*} \neg A_{s}$; so by $L_{3.2}, \Omega_{k-1} \cup\left\{A_{s}\right\}$ is consistent; so $\Omega_{k}$ is consistent.

(iii) Suppose $\Omega_{k}$ is $\Omega_{k^{*}} \cup\left\{\right.$ s.t, $\left.\neg P_{t}\right\}$. In this case, as above, $\Omega_{k^{*}}$ is consistent and by construction, $(\square \mathrm{Q} \wedge \neg \square \mathrm{P})_{s} \in \Omega_{\mathrm{k}^{*}}$. Suppose $\Omega_{k}$ is inconsistent. Then there are $A_{\mathfrak{u}}$ and $\neg A_{\mathfrak{u}}$ such that $\Omega_{k^{*}} \cup$ $\left\{\right.$ s.t, $\left.\neg P_{t}\right\} \vdash_{N N \alpha}^{*} A_{u}$ and $\Omega_{k^{*}} \cup\left\{\right.$ s.t, $\left.\neg P_{t}\right\} \vdash_{N N \alpha}^{*} \neg A_{u}$. So reason as follows,



where, by construction, $t$ is not in $\Omega_{\mathrm{k}^{*}}$. So $\Omega_{\mathrm{k}^{*}} \vdash_{\mathrm{NN \alpha}}^{*} \square \mathrm{P}_{s}$; but $(\square \mathrm{Q} \wedge \neg \square \mathrm{P})_{\mathrm{s}} \in \Omega_{\mathrm{k}^{*}}$; so with $(\wedge \mathrm{E}), \Omega_{\mathrm{k}^{*}} \vdash_{\mathrm{NN \alpha}}^{*} \neg \square \mathrm{P}_{\mathrm{s}}$; so $\Omega_{\mathrm{k}^{*}}$ is inconsistent. This is impossible; reject the assumption: $\Omega_{\mathrm{k}}$ is consistent.

For any $i, \Omega_{i}$ is consistent.

$\mathrm{L}_{3} .6$ If $\Gamma_{0}$ is consistent, then $\Gamma^{\prime}$ is consistent.

Suppose $\Gamma_{0}$ is consistent, but $\Gamma^{\prime}$ is not; from the latter, there is some $P_{s}$ such that $\Gamma^{\prime} \vdash_{\mathrm{NN} \alpha}^{*} P_{s}$ and $\Gamma^{\prime} \vdash_{\mathrm{NN} \alpha}^{*} \neg P_{s}$. Consider derivations Di and D2 of these results, and the premises $\mathcal{P}_{i} \ldots \mathcal{P}_{j}$ of these derivations. By construction, there is an $\Omega_{k}$ with each of these premises as a member; 
so Di and D2 are derivations from $\Omega_{k}$; so $\Omega_{k}$ is not consistent. But since $\Gamma_{0}$ is consistent, by $\mathrm{L}_{3.5}, \Omega_{\mathrm{k}}$ is consistent. This is impossible; reject the assumption: if $\Gamma_{0}$ is consistent then $\Gamma^{\prime}$ is consistent.

$\mathrm{L}_{3.7}$ If $\Gamma_{0}$ is consistent, then $\Gamma^{\prime}$ is a scapegoat set.

Suppose $\Gamma_{0}$ is consistent and $\Gamma^{\prime} \vdash_{\mathrm{NN \alpha}}^{*}(\square \mathrm{Q} \wedge \neg \square \mathrm{P})_{s}$. By $\mathrm{L}_{3} .6, \Gamma^{\prime}$ is consistent; and by the constraints on subscripts, $s$ is included in $\Gamma^{\prime}$. Since $\Gamma^{\prime}$ is consistent, $\Gamma^{\prime} H_{N N \alpha}^{*} \neg(\square \mathrm{Q} \wedge \neg \square \mathrm{P})_{s}$; so there is a stage in the construction process where $\Omega_{i^{*}}=\Omega_{i-1} \cup\left\{(\square \mathrm{Q} \wedge \neg \square \mathrm{P})_{s}\right\}$ and $\Omega_{i}=\Omega_{i^{*}} \cup\left\{\right.$ s.t, $\left.\neg \mathrm{P}_{t}\right\}$; so by construction, s.t $\in \Gamma^{\prime}$ and $\neg \mathrm{P}_{\mathrm{t}} \in \Gamma^{\prime}$; so $\Gamma^{\prime} \vdash_{\mathrm{NN \alpha}}^{*}$ s.t and $\Gamma^{\prime} \vdash_{\mathrm{NN} \alpha}^{*} \neg \mathrm{P}_{\mathrm{t}}$. So $\Gamma^{\prime}$ is a scapegoat set.

$\mathrm{C}$ (I) We construct an interpretation $\mathrm{I}=\langle\mathrm{W}, \mathrm{N}, \mathrm{R}, v\rangle$ based on $\Gamma^{\prime}$ as follows. Let $W$ have a member $w_{s}$ corresponding to each subscript $s$ included in $\Gamma^{\prime}$. Then set $w_{s} \in N$ iff there is some Q such that $\Gamma^{\prime} \vdash_{\mathrm{NN \alpha}}^{*} \square \mathrm{Q}_{s}$; set $\mathrm{R}=\left\{\left\langle w_{s}, w_{s}\right\rangle \mid w_{s} \in(\mathrm{W}-\mathrm{N})\right\} \cup\left\{\left\langle w_{s}, w_{\mathrm{t}}\right\rangle \mid \Gamma^{\prime} \vdash_{\mathrm{NN} \alpha}^{*}\right.$ s.t $\} ;$ and $v_{w_{s}}(\mathrm{p})=1$ iff $\Gamma^{\prime} \vdash_{\mathrm{N} \alpha}^{*} p_{s}$.

Note that $w_{0} \in N$. By a simple derivation, $\vdash_{\mathrm{NN \alpha}}^{*} \square T_{0}$; so $\Gamma^{\prime} \vdash_{\mathrm{NN} \alpha}^{*} \square T_{0}$; so $w_{0} \in N$.

$\mathrm{L}_{3} .8$ If $\Gamma_{0}$ is consistent then for $\langle\mathrm{W}, \mathrm{N}, \mathrm{R}, v\rangle$ constructed as above, and for any $s$ included in $\Gamma^{\prime}, v_{w_{s}}(A)=1$ iff $\Gamma^{\prime} \vdash_{\mathrm{NN} \alpha}^{*} A_{s}$.

Suppose $\Gamma_{0}$ is consistent and $s$ is included in $\Gamma^{\prime}$. By $\mathrm{L}_{3} \cdot 4, \Gamma^{\prime}$ is s-maximal. By $\mathrm{L}_{3} .6$ and $\mathrm{L}_{3.7}, \Gamma^{\prime}$ is consistent and a scapegoat set. Now by induction on the number of operators in $A_{s}$,

Basis: If $A_{s}$ has no operators, then it is a parameter $p_{s}$ and by construction, $v_{w_{s}}(p)=1$ iff $\Gamma^{\prime} \vdash_{\mathrm{NN} \alpha}^{*} p_{s}$. So $v_{w_{s}}(A)=1$ iff $\Gamma^{\prime} \vdash_{\mathrm{NN} \alpha}^{*} A_{s}$.

Assp: For any $i, 0 \leqslant i<k$, if $A_{s}$ has $i$ operators, then $v_{w_{s}}(A)=1$ iff $\Gamma^{\prime} \vdash_{\mathrm{NN} \alpha}^{*} A_{s}$.

Show: If $A_{s}$ has $k$ operators, then $v_{w_{s}}(A)=1$ iff $\Gamma^{\prime} \vdash_{\mathrm{N} N \alpha}^{*} A_{s}$.

If $A_{s}$ has $k$ operators, then it is of the form $\neg P_{s},(P \supset Q)_{s}$, $(P \wedge Q)_{s},(P \vee Q)_{s},(P \equiv Q)_{s}, \square P_{s}$ or $\diamond P_{s}$ where $P$ and $Q$ have $<k$ operators.

$(\neg) A_{s}$ is $\neg P_{s}$. (i) Suppose $v_{w_{s}}(A)=1$; then $v_{w_{s}}(\neg P)=1$; so by $\mathrm{TN}(\neg), v_{w_{s}}(\mathrm{P})=0$; so by assumption, $\Gamma^{\prime} \nvdash_{\mathrm{NN} \alpha}^{*} \mathrm{P}_{s}$; so by $s-$ maximality, $\Gamma^{\prime} \vdash_{\mathrm{NN \alpha}}^{*} \neg \mathrm{P}_{s}$, where this is to say, $\Gamma^{\prime} \vdash_{\mathrm{NN \alpha}}^{*} A_{s}$. (ii) Suppose $\Gamma^{\prime} \vdash_{\mathrm{NN \alpha}}^{*} A_{s}$; then $\Gamma^{\prime} \vdash_{\mathrm{NN \alpha}}^{*} \neg \mathrm{P}_{s}$; so by consistency, $\Gamma^{\prime} \nvdash_{\mathrm{NN \alpha}}^{*} \mathrm{P}_{s}$; so by assumption, $v_{w_{s}}(\mathrm{P})=0$; so by $\mathrm{TN}(\neg), v_{w_{s}}(\neg \mathrm{P})=1$, where this is to say, $v_{w_{s}}(A)=1$. So $v_{w_{s}}(A)=1$ iff $\Gamma^{\prime} \vdash_{\mathrm{NN} \alpha}^{*} A_{s}$.

(つ) $A_{s}$ is $(P \supset Q)_{s}$. (i) Suppose $v_{w_{s}}(A)=1$ but $\Gamma^{\prime} H_{N N \alpha}^{*} A_{s}$; then $v_{w_{s}}(\mathrm{P} \supset \mathrm{Q})=1$ but $\Gamma^{\prime} H_{N N \alpha}^{*}(\mathrm{P} \supset \mathrm{Q})_{s}$. From the latter, by smaximality, $\Gamma^{\prime} \vdash_{\mathrm{NN \alpha}}^{*} \neg(\mathrm{P} \supset \mathrm{Q})_{s}$; from this it follows, by simple 
derivations, that $\Gamma^{\prime} \vdash_{\mathrm{NN} \alpha}^{*} \mathrm{P}_{s}$ and $\Gamma^{\prime} \vdash_{\mathrm{NN} \alpha}^{*} \neg \mathrm{Q}_{s}$; so by consistency, $\Gamma^{\prime} \nvdash_{\mathrm{N} N \alpha}^{*} \mathrm{Q}_{s}$; so by assumption, $v_{w_{s}}(\mathrm{P})=1$ and $v_{w_{s}}(\mathrm{Q})=0$; so by $\mathrm{TN}(\supset), v_{w_{s}}(\mathrm{P} \supset \mathrm{Q})=0$. This is impossible; reject the assumption: if $v_{w_{s}}(A)=1$ then $\Gamma^{\prime} \vdash_{\mathrm{NN} \alpha}^{*} A_{s}$.

(ii) Suppose $\Gamma^{\prime} \vdash_{\mathrm{NN \alpha}}^{*} A_{s}$ but $v_{w_{s}}(A)=0$; then $\Gamma^{\prime} \vdash_{\mathrm{NN} \alpha}^{*}(\mathrm{P} \supset \mathrm{Q})_{s}$ but $v_{w_{s}}(\mathrm{P} \supset \mathrm{Q})=0$. From the latter, by $\mathrm{TN}(\supset), v_{w_{s}}(\mathrm{P})=1$ and $v_{w_{s}}(\mathrm{Q})=0$; so by assumption, $\Gamma^{\prime} \vdash_{\mathrm{NN \alpha}}^{*} \mathrm{P}_{\mathrm{s}}$ and $\Gamma^{\prime} \vdash_{\mathrm{NN \alpha}}^{*} \mathrm{Q}_{s}$; but since $\Gamma^{\prime} \vdash_{\mathrm{NN} \alpha}^{*}(\mathrm{P} \supset \mathrm{Q})_{s}$ and $\Gamma^{\prime} \vdash_{\mathrm{NN} \alpha}^{*} \mathrm{P}_{s}$, by $(\supset \mathrm{E}), \Gamma^{\prime} \vdash_{\mathrm{NN} \alpha}^{*} \mathrm{Q}_{s}$. This is impossible; reject the assumption: if $\Gamma^{\prime} \vdash_{\mathrm{NN \alpha}}^{*} A_{s}$, then $v_{w_{s}}(A)=1$. So $v_{w_{s}}(A)=1$ iff $\Gamma^{\prime} \vdash_{\mathrm{NN} \alpha}^{*} A_{s}$.

$(\wedge)$

( $\square) A_{s}$ is $\square P_{s}$. (i) Suppose $v_{w_{s}}(A)=1$ but $\Gamma^{\prime} \nvdash_{N N \alpha}^{*} A_{s}$; then $v_{w_{s}}(\square P)=$ 1 but $\Gamma^{\prime} \nvdash_{\mathrm{NN} \alpha}^{*} \square \mathrm{P}_{s}$. From the former, by $\mathrm{TN}(\square), w_{s} \in \mathrm{N}$; so by construction, there is some Q such that $\Gamma^{\prime} \vdash_{\mathrm{NN \alpha}}^{*} \square \mathrm{Q}_{s}$; from the latter, by s-maximality, $\Gamma^{\prime} \vdash_{\mathrm{NN} \alpha}^{*} \neg \square \mathrm{P}_{s}$; so by $(\wedge \mathrm{I}), \Gamma^{\prime} \vdash_{\mathrm{NN} \alpha}^{*}$ $(\square \mathrm{Q} \wedge \neg \square \mathrm{P})_{s}$; so, since $\Gamma^{\prime}$ is a scapegoat set, there is some $\mathrm{t}$ such that $\Gamma^{\prime} \vdash_{\mathrm{NN \alpha}}^{*}$ s.t and $\Gamma^{\prime} \vdash_{\mathrm{NN \alpha}}^{*} \neg P_{t}$; from the first, by construction, $\left\langle w_{s}, w_{t}\right\rangle \in R$; and from the second, by consistency, $\Gamma^{\prime} H_{N N \alpha}^{*} P_{t}$; so by assumption, $v_{w_{t}}(P)=0$; but $w_{s} R w_{t}$; so by $\mathrm{TN}(\square), v_{w_{s}}(\square \mathrm{P})=0$. This is impossible; reject the assumption: if $v_{w_{s}}(A)=1$, then $\Gamma^{\prime} \vdash_{\mathrm{NN} \alpha}^{*} A_{s}$.

(ii) Suppose $\Gamma^{\prime} \vdash_{\mathrm{NN} \alpha}^{*} A_{s}$ but $v_{w_{s}}(A)=0$; then $\Gamma^{\prime} \vdash_{\mathrm{NN \alpha}}^{*} \square \mathrm{P}_{s}$ but $v_{w_{s}}(\square \mathrm{P})=0$. From the former, by construction, $w_{\mathrm{s}} \in \mathrm{N}$; so with the latter, by $\mathrm{TN}(\square)$, there is some $w_{\mathrm{t}} \in W$ such that $w_{\mathrm{s}} \mathrm{R} w_{\mathrm{t}}$ and $v_{w_{t}}(P)=0$; so by assumption, $\Gamma^{\prime} H_{N N \alpha}^{*} P_{t}$; but since $w_{s} R w_{t}$ and $w_{\mathrm{s}} \in \mathrm{N}$, by construction, $\Gamma^{\prime} \vdash_{\mathrm{NN} \alpha}^{*}$ s.t; so by $(\square \mathrm{E}), \Gamma^{\prime} \vdash_{\mathrm{NN} \alpha}^{*} P_{\mathrm{t}}$. This is impossible; reject the assumption: if $\Gamma^{\prime} \vdash_{\mathrm{NN \alpha}} A_{s}$ then $v_{w_{s}}(A)=1$. So $v_{w_{s}}(A)=1$ iff $\Gamma^{\prime} \vdash_{N N \alpha}^{*} A_{s}$.

$(\diamond) A_{s}$ is $\diamond P_{s}$. (i) Suppose $v_{w_{s}}(A)=1$ but $\Gamma^{\prime} \nvdash_{N N \alpha}^{*} A_{s}$; then $v_{w_{s}}(\diamond P)=$ 1 but $\Gamma^{\prime} H_{\mathrm{NN} \alpha}^{*} \diamond \mathrm{P}_{s}$; from the latter, by s-maximality, $\Gamma^{\prime} \vdash_{\mathrm{NN} \alpha}^{*} \neg \diamond \mathrm{P}_{s}$; so by (MN), $\Gamma^{\prime} \vdash_{\mathrm{NN} \alpha}^{*} \square \neg \mathrm{P}_{s}$; so by construction, $w_{\mathrm{s}} \in \mathrm{N}$; so, with the former, by $\mathrm{TN}(\diamond)$, there is some $w_{\mathrm{t}} \in \mathrm{W}$ such that $w_{\mathrm{s}} \mathrm{R} w_{\mathrm{t}}$ and $v_{w_{\mathrm{t}}}(\mathrm{P})=1$; so by assumption, $\Gamma^{\prime} \vdash_{\mathrm{NN} \alpha}^{*} \mathrm{P}_{\mathrm{t}}$; but since $w_{\mathrm{s}} \mathrm{R} w_{\mathrm{t}}$ and $w_{s} \in \mathrm{N}$, by construction, $\Gamma^{\prime} \vdash_{\mathrm{NN} \alpha}^{*}$ s.t; so by $(\diamond \mathrm{I}), \Gamma^{\prime} \vdash_{\mathrm{NN} \alpha}^{*} \diamond \mathrm{P}_{s}$. This is impossible; reject the assumption: if $v_{w_{s}}(A)=1$ then $\Gamma^{\prime} \vdash_{\mathrm{NN} \alpha}^{*} A_{s}$.

(ii) Suppose $\Gamma^{\prime} \vdash_{\mathrm{NN} \alpha}^{*} A_{s}$ but $v_{w_{s}}(A)=0$; then $\Gamma^{\prime} \vdash_{\mathrm{NN} \alpha}^{*} \diamond \mathrm{P}_{s}$ but $v_{w_{s}}(\diamond \mathrm{P})=0$. From the latter, by $\mathrm{TN}(\diamond), w_{s} \in \mathrm{N}$; so by construction, there is some $\mathrm{Q}$ such that $\Gamma^{\prime} \vdash_{\mathrm{NN \alpha}}^{*} \square \mathrm{Q}_{s}$; from the former, by $(\mathrm{MN}), \Gamma^{\prime} \vdash_{\mathrm{NN} \alpha}^{*} \neg \square \neg \mathrm{P}_{s}$; so by $(\wedge \mathrm{I}), \Gamma^{\prime} \vdash_{\mathrm{NN} \alpha}^{*}(\square \mathrm{Q} \wedge$ 
$\neg \square \neg P)_{s}$; so, since $\Gamma^{\prime}$ is a scapegoat set, there is some $t$ such that $\Gamma^{\prime} \vdash_{\mathrm{NN \alpha}}^{*}$ s.t and $\Gamma^{\prime} \vdash_{\mathrm{NN} \alpha}^{*} \neg \neg P_{\mathrm{t}}$; from the first, by construction, $\left\langle w_{s}, w_{t}\right\rangle \in R$; from the second, by (DN), $\Gamma^{\prime} \vdash_{\mathrm{NN \alpha}}^{*} \mathrm{P}_{\mathrm{t}}$; so by assumption, $v_{w_{t}}(\mathrm{P})=1$; so since $w_{\mathrm{s}} \mathrm{R} w_{\mathrm{t}}$ by $\mathrm{TN}(\diamond), v_{w_{s}}(\diamond \mathrm{P})=1$. This is impossible; reject the assumption: if $v_{w_{s}}(A)=1$ then $\Gamma^{\prime} \vdash_{\mathrm{NN} \alpha}^{*} A_{s}$. So $v_{w_{s}}(A)=1$ iff $\Gamma^{\prime} \vdash_{\mathrm{NN \alpha}}^{*} A_{s}$.

For any $A_{s}, v_{w_{s}}(A)=1$ iff $\Gamma^{\prime} \vdash_{\mathrm{NN} \alpha}^{*} A_{s}$.

$\mathrm{L}_{3.9}$ If $\Gamma_{0}$ is consistent, then $\langle W, N, R, v\rangle$ constructed as above is an $N \alpha$ interpretation.

In each case, we need to show that the interpretation meets the condition(s) $\alpha$. Suppose $\Gamma_{0}$ is consistent.


construction, $\left\langle w_{s}, w_{s}\right\rangle \in R$ and $\eta$ is satisfied. So suppose $w_{s} \in$ $\mathrm{N}$. Then by construction, there is some $\mathrm{Q}$ such that $\Gamma^{\prime} \vdash_{\mathrm{NN} \alpha}^{*} \square \mathrm{Q}_{s}$; so by reasoning as follows,

\begin{tabular}{|c|c|c|}
\hline \multicolumn{3}{|c|}{$\Gamma^{\prime}$} \\
\hline & $\square \mathrm{Q}_{\mathrm{s}}$ & from $\Gamma^{\prime}$ \\
\hline & s.t & $\mathrm{A}(\mathrm{g}, \mathrm{AM \eta})$ \\
\hline & $T_{t}$ & $T$ is a tautology \\
\hline & $\diamond T_{s}$ & $3,4 \diamond \mathrm{I}$ \\
\hline 6 & $\diamond T_{s}$ & 3-5 AMn \\
\hline & $\neg \square \neg \top_{\mathrm{s}}$ & $6 \mathrm{MN}$ \\
\hline & $(\square \mathrm{Q} \wedge \neg \square \neg \mathrm{T})_{s}$ & $2,7 \wedge \mathrm{I}$ \\
\hline
\end{tabular}

$\Gamma^{\prime} \vdash_{\mathrm{NN} \alpha}^{*}(\square \mathrm{Q} \wedge \neg \square \neg \top)_{s}$; but by $\mathrm{L}_{3.7}, \Gamma^{\prime}$ is a scapegoat set; so there is a $t$ such that $\Gamma^{\prime} \vdash_{\mathrm{NN} \alpha}^{*}$ s.t; so by construction, $\left\langle w_{s}, w_{t}\right\rangle \in R$ and $\eta$ is satisfied.

( $\rho$ ) Suppose $\alpha$ includes condition $\rho$ and $w_{s} \in W$. Then by construction, $s$ is a subscript in $\Gamma^{\prime}$; so by $(\mathrm{AM} \rho), \Gamma^{\prime} \vdash_{\mathrm{NN \alpha}}^{*}$ s.s; so by construction, $\left\langle w_{s}, w_{s}\right\rangle \in R$ and $\rho$ is satisfied.

( $\sigma)$ Suppose $\alpha$ includes condition $\sigma$ and $\left\langle w_{s}, w_{t}\right\rangle \in R$. If $w_{s}=w_{t}$ then $\sigma$ is satisfied automatically. So suppose $w_{s} \neq w_{t}$; then by construction, $\Gamma^{\prime} \vdash_{\mathrm{NN} \alpha}^{*}$ s.t; so by $(\mathrm{AM} \sigma), \Gamma^{\prime} \vdash_{\mathrm{NN} \alpha}^{*}$ t.s; so by construction, $\left\langle w_{\mathrm{t}}, w_{\mathrm{s}}\right\rangle \in \mathrm{R}$ and $\sigma$ is satisfied.

( $\tau$ ) Suppose $\alpha$ includes condition $\tau$ and $\left\langle w_{s}, w_{t}\right\rangle,\left\langle w_{t}, w_{u}\right\rangle \in R$. If $w_{\mathrm{s}}=w_{\mathrm{t}}$ or $w_{\mathrm{t}}=w_{\mathrm{u}}$, then $\tau$ is satisfied automatically. So suppose $w_{s} \neq w_{t}$ and $w_{t} \neq w_{u}$; then by construction, $\Gamma^{\prime} \vdash_{\mathrm{NN} \alpha}^{*}$ s.t and $\Gamma^{\prime} \vdash_{\mathrm{NN \alpha}}^{*}$ t.u; so by $(\mathrm{AM} \tau), \Gamma^{\prime} \vdash_{\mathrm{NN \alpha}}^{*}$ s.u; so by construction, $\left\langle w_{s}, w_{\mathfrak{u}}\right\rangle \in R$ and $\tau$ is satisfied.

MAP For any $w_{s} \in W$, set $m(s)=w_{s}$; otherwise $m(s)$ is arbitrary. 
L3.IO If $\Gamma_{0}$ is consistent, then $v_{m}\left(\Gamma_{0}\right)=1$.

Suppose $\Gamma_{0}$ is consistent and $A_{0} \in \Gamma_{0}$; then by construction, $A_{0} \in \Gamma^{\prime}$; so $\Gamma^{\prime} \vdash_{\mathrm{NN} \alpha}^{*} A_{0}$; so since $\Gamma_{0}$ is consistent, by $\mathrm{L}_{3} .8, v_{w_{0}}(A)=1$. And similarly for any $A_{0} \in \Gamma_{0}$. But $m(0)=w_{0}$; so $v_{m}\left(\Gamma_{0}\right)=1$.

Main result: Suppose $\Gamma \models_{N \alpha} A$ but $\Gamma \nvdash_{N N \alpha} A$. Then $\Gamma_{0} \models_{N \alpha \alpha}^{*} A_{0}$ but $\Gamma_{0} \nvdash_{N N \alpha}^{*} A_{0}$. By (DN), if $\Gamma_{0} \vdash_{\mathrm{NN} \alpha}^{*} \neg \neg A_{0}$, then $\Gamma_{0} \vdash_{\mathrm{NN} \alpha}^{*} A_{0}$; so $\Gamma_{0} \nvdash_{\mathrm{NN} \alpha}^{*} \neg \neg A_{0}$; so by $L_{3.2}, \Gamma_{0} \cup\left\{\neg A_{0}\right\}$ is consistent; so by $\mathrm{L}_{3} .9$ and $\mathrm{L}_{3} . \mathrm{IO}$, there is an $\mathrm{N} \alpha$ interpretation $\langle\mathrm{W}, \mathrm{N}, \mathrm{R}, v\rangle_{\mathrm{m}}$ constructed as above such that $v_{m}\left(\Gamma_{0} \cup\left\{\neg A_{0}\right\}\right)=1$; so $v_{m(0)}(\neg A)=1$; so by $\mathrm{TN}(\neg), v_{\mathrm{m}(0)}(A)=0$; so $v_{\mathrm{m}}\left(\Gamma_{0}\right)=1$ and $v_{\mathrm{m}(0)}(A)=0$; so by $\mathrm{VN} \alpha^{*}$, $\Gamma_{0} \nvdash_{N_{\alpha}}^{*} A_{0}$. This is impossible; reject the assumption: if $\Gamma \models_{N \alpha} A$, then $\Gamma \vdash_{\mathrm{NN \alpha}} A$.

4 Conditional logics: $C x(\mathrm{CH} .5)$

\section{I LANGUAGE / SEMANTIC NOTIONS}

LCx The vocabulary consists of propositional parameters $p_{0}, p_{1} \ldots$ with the operators, $\neg, \wedge, \vee, \supset, \equiv, \square, \diamond$ and $>$. Each propositional parameter is a FORMULA; if $A$ and $B$ are formulas, so are $\neg A,(A \wedge B),(A \vee B)$, $(A \supset B),(A \equiv B), \square A, \diamond A$ and $(A>B)$.

ICx Where $\mathfrak{I}$ is the set of all formulas in the language, an INTER PRETATION is $\left\langle W,\left\{R_{A} \mid A \in \mathfrak{I}\right\}, v\right\rangle$ where $W$ is a set of worlds, and $v$ assigns 0 or 1 to parameters at worlds. The middle term is a set of access relations: for any formula $A$, there is an access relation $R_{A}$ which says which worlds are $A$-accessible from any $w$. Say $f_{A}(w)=\left\{x \in W \mid w R_{A} x\right\}$, and $[A]=$ $\left\{w \mid v_{w}(A)=1\right\}$. Then, where $x$ is empty or indicates some combination of the following constraints,

(I) $f_{A}(w) \subseteq[A]$

(2) If $w \in[A]$, then $w \in f_{A}(w)$

(3) If $[A] \neq \phi$, then $f_{A}(w) \neq \phi$

(4) If $\mathrm{f}_{\mathrm{A}}(w) \subseteq[B]$ and $\mathrm{f}_{\mathrm{B}}(w) \subseteq[A]$, then $\mathrm{f}_{\mathrm{A}}(w)=\mathrm{f}_{\mathrm{B}}(w)$

(5) If $f_{A}(w) \cap[B] \neq \phi$, then $f_{A \wedge B}(w) \subseteq f_{A}(w)$

(6) If $x \in f_{A}(w)$ and $y \in f_{A}(w)$, then $x=y$

(7) If $x \in[A]$, and $y \in f_{A}(x)$, then $x=y$

$\left\langle\mathrm{W},\left\{\mathrm{R}_{\mathrm{A}} \mid A \in \mathfrak{I}\right\}, v\right\rangle$ is a $C x$ interpretation when it meets the constraints from $x$. System $C$ has none of the extra constraints; $C+$ is $C$ with constraints (I) - (2); $C S$ is $C$ with constraints (I) - (5); $C_{I}$ is $C$ with constraints (I) - (5) and (7); $C_{2}$ is $C$ with constraints (I) - (5) and (6).

TC For complex expressions,

$(\neg) v_{w}(\neg A)=1$ if $v_{w}(A)=0$, and 0 otherwise. 
$(\wedge) v_{w}(A \wedge B)=1$ if $v_{w}(A)=1$ and $v_{w}(B)=1$, and 0 otherwise.

$(\vee) v_{w}(A \vee B)=1$ if $v_{w}(A)=1$ or $v_{w}(B)=1$, and 0 otherwise.

( $) v_{w}(\mathrm{~A} \supset \mathrm{B})=1$ if $v_{w}(\mathrm{~A})=0$ or $v_{w}(\mathrm{~B})=1$, and 0 otherwise.

$(\equiv) v_{w}(\mathrm{~A} \equiv \mathrm{B})=1$ if $v_{w}(\mathrm{~A})=v_{w}(\mathrm{~B})$, and 0 otherwise.

$(\diamond)_{v} v_{w}(\diamond A)=1$ if some $x \in W$ has $v_{x}(A)=1$, and 0 otherwise.

$(\square)_{v} v_{w}(\square A)=1$ if all $x \in W$ have $v_{x}(A)=1$, and 0 otherwise.

(>) $v_{w}(\mathrm{~A}>\mathrm{B})=1$ iff all $x \in W$ such that $w \mathrm{R}_{\mathrm{A}} \times$ have $v_{x}(\mathrm{~B})=1$.

For a set $\Gamma$ of formulas, $v_{w}(\Gamma)=1$ iff $v_{w}(A)=1$ for each $A \in \Gamma$; then,

$\operatorname{VCx} \Gamma \models_{C_{x}} A$ iff there is no $C x$ interpretation $\left\langle W,\left\{R_{A} \mid A \in \mathfrak{I}\right\}, v\right\rangle$ and $w \in W$ such that $v_{w}(\Gamma)=1$ and $v_{w}(A)=0$.

\subsection{NATURAL DERIVATIONS: $N C x$}

Derivation systems $N C x$ take over $\neg, \supset, \wedge, \vee, \equiv, \square$ and $\diamond$ rules from $N K v$. Thus modal rules are,
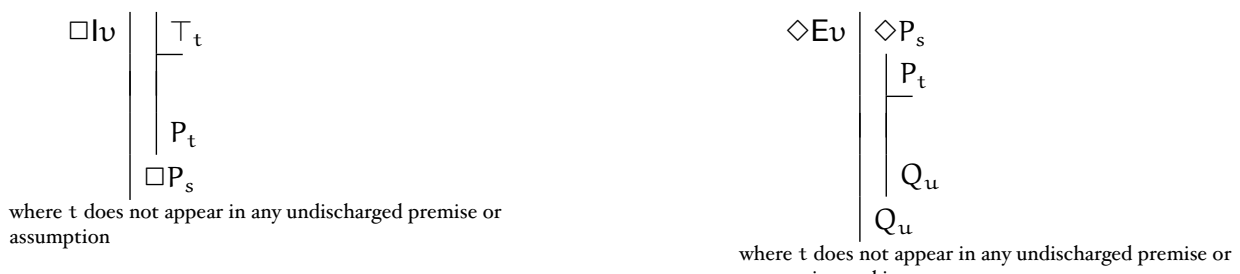

$$
\square \mathrm{Ev} \mid \begin{aligned}
& \square \mathrm{P}_{\mathrm{s}} \\
& \mathrm{P}_{\mathrm{t}}
\end{aligned}
$$

For $>$, let there be new subscripted expressions of the sort $A_{s / t}-$ which intuitively say $w_{s} R_{A} w_{t}$. Expressions of this sort do not interact with other formulas except as follows (and so do not interact with rules of $N K v$ ):
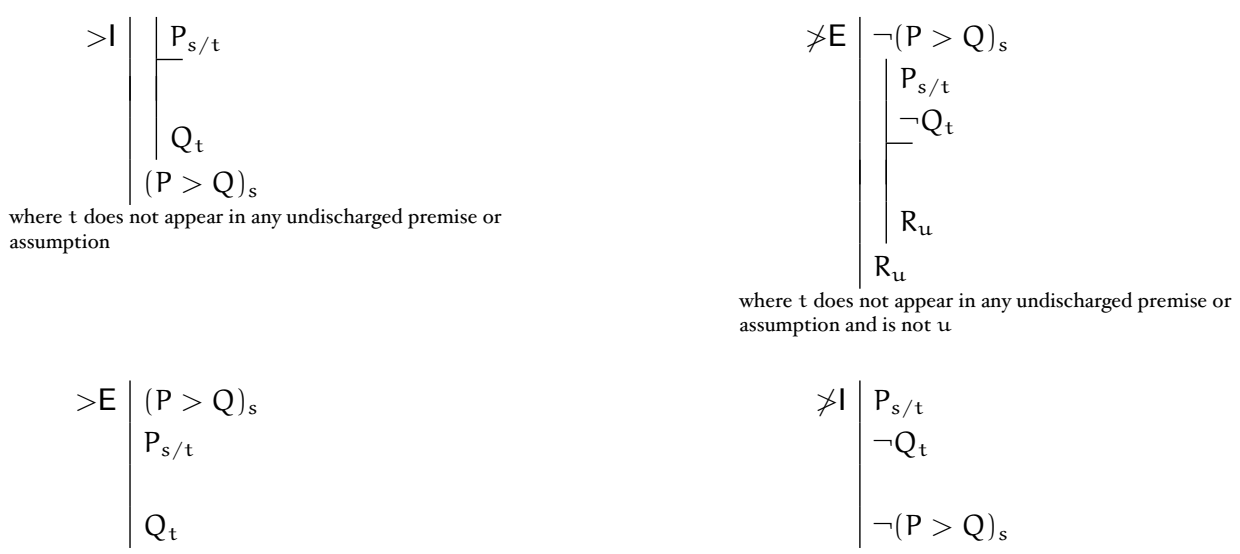
Corresponding to constraints (I) - (7) are AMP1, $\mathrm{AMP}_{2}, \mathrm{AMs}_{1}, \mathrm{AMs}_{2}, \mathrm{AMs}_{3}$, AMRs, and two forms of AMDL. For AMRS $\mathcal{A}_{(t)}$ is an expression of the sort $\mathrm{Q}_{\mathrm{t}}, \mathrm{Q}_{\mathrm{t} / v}, \mathrm{Q}_{v / \mathrm{t}}$ or $\mathrm{Q}_{\mathrm{t} / \mathrm{t}}$ with a subscript $\mathrm{t}$, and $\mathcal{A}_{(\mathrm{u})}$ is like $\mathcal{A}_{(\mathrm{t})}$ except that some instance(s) of $t$ are replaced by $u$. And similarly for AMDL.

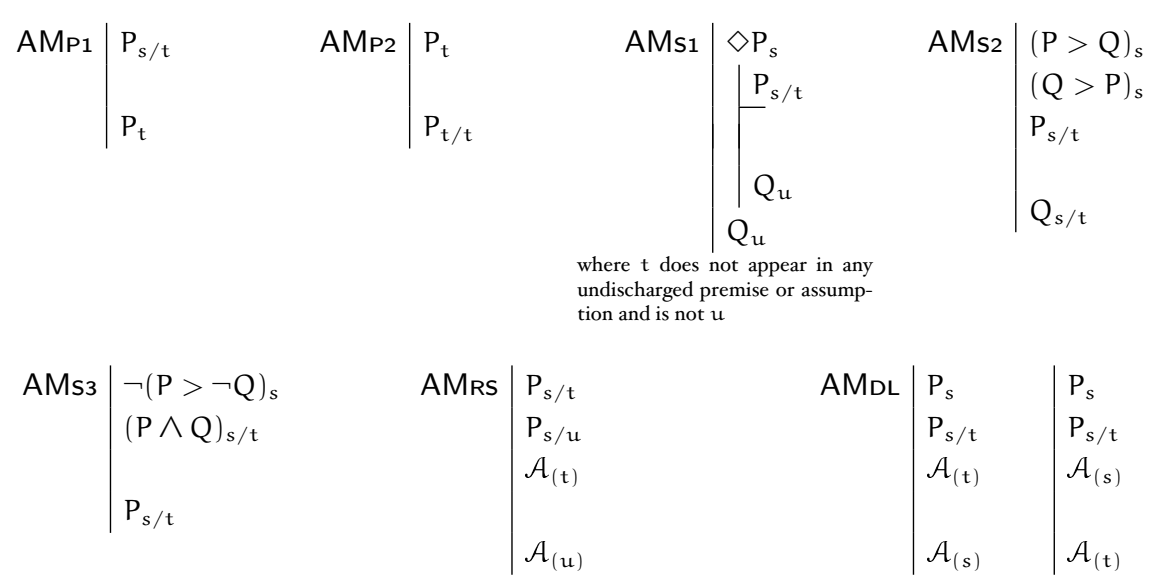

In these systems, every subscript is 0 , appears in a premise, or appears in the $\mathrm{t}$-place of an assumption for $\square \mathrm{I} v, \diamond \mathrm{Ev},>\mathrm{I}, \ngtr \mathrm{E}$ or AMs. Intuitively there are plus rules, rules for the sphere conception, and rules for the Stalnaker and Lewis alternatives. $N C$ includes just the rules of $N K v$ plus $>\mathrm{I},>\mathrm{E}, \ngtr \mathrm{I}$ and $\ngtr \mathrm{E}$ (but, as below, the latter two are derived). Then,

$N C+$ has the rules of $N C$ plus $\mathrm{AMP}_{1}, \mathrm{AMP}_{2}$

$N C S$ has the rules of $N C$ plus $\mathrm{AMP}_{1}, \mathrm{AMP}_{2}, \mathrm{AMs}_{1}, \mathrm{AMs}_{2}, \mathrm{AMs}_{3}$

$N C_{I}$ has the rules of $N C$ plus $\mathrm{AMP}_{1}, \mathrm{AMP}_{2}, \mathrm{AMs}_{1}, \mathrm{AMs}_{2}, \mathrm{AMs}_{3}, \mathrm{AMDL}$

$\mathrm{NC}_{2}$ has the rules of $N C$ plus $\mathrm{AMP}_{1}, \mathrm{AMP}_{2}, \mathrm{AMs}_{1}, \mathrm{AMs}_{2}, \mathrm{AMs}_{3}, \mathrm{AMRS}$

Where $\Gamma$ is a set of unsubscripted formulas, let $\Gamma_{0}$ be those same formulas each with subscript 0 . Then,

$\mathrm{NCx} \Gamma \vdash_{\mathrm{NCx}} A$ iff there is an $N C x$ derivation of $A_{0}$ from $\Gamma_{0}$.

Derived rules carry over from $N K \alpha$. In addition, as first examples, $\ngtr \mathrm{I}$ and $\ngtr \mathrm{E}$ are derived rules in $N C$, and so in any $N C x$. 


\begin{tabular}{|c|c|c|}
\hline \\
\hline & $P_{s / t}$ & $\mathrm{P}$ \\
\hline & $\neg \mathrm{Q}_{\mathrm{t}}$ & $\mathrm{P}$ \\
\hline & $(P>Q)_{s}$ & $\mathrm{~A}(c, \neg \mathrm{I})$ \\
\hline & $Q_{t}$ & $\mathrm{I}, 3>\mathrm{E}$ \\
\hline & $\neg Q_{t}$ & $2 \mathrm{R}$ \\
\hline & $\neg(\mathrm{P}>\mathrm{Q})_{s}$ & $3-5 \neg \mathrm{I}$ \\
\hline
\end{tabular}



As final examples, here is a case in $N C S$ using $\mathrm{AMs}_{3}$ and then again in $\mathrm{NC}_{2}$ but without appeal to $\mathrm{AMs}_{3}$ (so that $\mathrm{AMs}_{3}$ is not necessary in $\mathrm{NC}_{2}$ for the result). This last case is a bit messy, but should nicely illustrate use of the rules.

\begin{tabular}{|c|c|c|}
\hline \multicolumn{3}{|c|}{$A>B, \neg(A>\neg C) \vdash_{N C S}(A \wedge C)>B$} \\
\hline I & $(A>B)_{0}$ & $\mathrm{P}$ \\
\hline 2 & $\neg(A>\neg C)_{0}$ & $\mathrm{P}$ \\
\hline 3 & $(A \wedge C)_{0 / 1}$ & $\mathrm{~A}(g,>\mathrm{I})$ \\
\hline 4 & $A_{0 / 1}$ & $2,3 \mathrm{AMs}_{3}$ \\
\hline 5 & $\mathrm{~B}_{1}$ & $\mathrm{I}, 4>\mathrm{E}$ \\
\hline 6 & {$[(A \wedge C)>B]_{0}$} & $3-5>I$ \\
\hline
\end{tabular}

\begin{tabular}{|c|c|c|}
\hline \multicolumn{3}{|c|}{$A>B, \neg(A>\neg C) \vdash_{N C 2}(A \wedge C)>B$} \\
\hline I & $(A>B)_{0}$ & $\mathrm{P}$ \\
\hline 2 & $\neg(A>\neg C)_{0}$ & $\mathrm{P}$ \\
\hline 3 & $A_{0 / 1}$ & $\mathrm{~A}(g, 2 \ngtr \mathrm{E})$ \\
\hline 4 & $\neg \neg C_{1}$ & \\
\hline 5 & $(A \wedge C)_{0 / 2}$ & $\mathrm{~A}(g,>\mathrm{I})$ \\
\hline 6 & $(A \wedge C)_{0 / 3}$ & $\mathrm{~A}(g,>\mathrm{I})$ \\
\hline 7 & $(A \wedge C)_{3}$ & $6 \mathrm{AMP}_{\mathrm{I}}$ \\
\hline 8 & $A_{3}$ & $7 \wedge \mathrm{E}$ \\
\hline 9 & {$[(A \wedge C)>A]_{0}$} & $6-8>\mathrm{I}$ \\
\hline IO & $A_{0 / 3}$ & $\mathrm{~A}(g,>\mathrm{I})$ \\
\hline II & $A_{3}$ & IO $A M P_{\text {I }}$ \\
\hline I2 & $\neg \neg C_{3}$ & 3,IO,4 AMrs \\
\hline $\mathrm{I} 3$ & $\mathrm{C}_{3}$ & I2 DN \\
\hline I4 & $(A \wedge C)_{3}$ & $\mathrm{II}, \mathrm{I} 3 \wedge \mathrm{I}$ \\
\hline I5 & {$[A>(A \wedge C)]_{0}$} & IO-I4 $>$ I \\
\hline 16 & $A_{0 / 2}$ & $9,15,5 \mathrm{AMs}_{2}$ \\
\hline I7 & $\mathrm{B}_{2}$ & $\mathrm{I}, \mathrm{I} 6>\mathrm{E}$ \\
\hline I8 & {$[(A \wedge C)>B]_{0}$} & $5-\mathrm{I} 7>\mathrm{I}$ \\
\hline I9 & {$[(A \wedge C)>B]_{0}$} & $2,3-\mathrm{I} 8 \ngtr \mathrm{E}$ \\
\hline
\end{tabular}

The derivation on the left is a simple application of $\mathrm{AMs}_{3}$. On the right, we go for the final goal by $\ngtr \mathrm{E} .{ }^{4}$ The real work is getting $A_{0 / 2}$ so that we can use $>E$ with (I). And we go for this by getting the conditionals that feed into AMs2, given that we already have $(A \wedge C)_{0 / 2}$.

\footnotetext{
${ }^{4}$ As, given strategies from [I2, chapter 6], we would jump on $\vee \mathrm{E}, \exists \mathrm{E}$ or $\diamond \mathrm{E}$ when available.
} 


\subsection{SOUNDNESS AND COMPLETENESS}

Preliminaries: Begin with generalized notions of validity. For a model $\left\langle W,\left\{R_{A} \mid\right.\right.$ $A \in \mathfrak{I}\}, v\rangle$, let $m$ be a map from subscripts into $W$. Say $\left\langle W,\left\{R_{A} \mid A \in \mathfrak{I}\right\}, v\right\rangle_{m}$ is $\left\langle W,\left\{R_{A} \mid A \in \mathfrak{I}\right\}, v\right\rangle$ with map $m$. Then, where $\Gamma$ is a set of expressions of our language for derivations, $v_{m}(\Gamma)=1$ iff for each $A_{s} \in \Gamma, v_{m}(s)(A)=1$, and for each $A_{s / t} \in \Gamma, m(t) \in f_{A}(m(s))$. Now expand notions of validity to include subscripted formulas, and alternate expressions as indicated in double brackets.

VCx ${ }^{*} \Gamma \models_{C x}^{*} A_{s} \llbracket A_{s / t} \rrbracket$ iff there is no $C x$ interpretation $\left\langle W,\left\{R_{A} \mid A \in \mathfrak{I}\right\}, v\right\rangle_{m}$ such that $v_{m}(\Gamma)=1$ but $v_{m(s)}(A)=0 \llbracket m(t) \notin f_{A}(m(s)) \rrbracket$.

$\mathrm{NCx}^{*} \Gamma \vdash_{\mathrm{NCx}}^{*} A_{s} \llbracket A_{s / t} \rrbracket$ iff there is an $N C x$ derivation of $A_{s} \llbracket A_{s / t} \rrbracket$ from the members of $\Gamma$.

These notions reduce to the standard ones when all the members of $\Gamma$ and $A$ have subscript 0 (and so do not include expressions of the sort $A_{s / t}$ ). This is obvious for $\mathrm{NCx}^{*}$. In the other case, there is a $\left\langle\mathrm{W},\left\{\mathrm{R}_{\mathrm{A}} \mid A \in \mathfrak{I}\right\}, v\right\rangle_{\mathrm{m}}$ and $w \in W$ that makes all the members of $\Gamma_{0}$ true and $A_{0}$ false just in case there is a world in $W$ that makes the unsubscripted members of $\Gamma$ true and $A$ false. For the following, cases omitted are like ones worked, and so left to the reader.

THEOREM 4.I $N C x$ is sound: If $\Gamma \vdash_{\mathrm{NCx}}$ A then $\Gamma \models_{\mathrm{Cx}_{\mathrm{x}}}$ A.

$$
\text { L4.I If } \Gamma \subseteq \Gamma^{\prime} \text { and } \Gamma \models_{C x}^{*} P_{s} \llbracket P_{s / t} \rrbracket \text {, then } \Gamma^{\prime} \models_{C x}^{*} P_{s} \llbracket P_{s / t} \rrbracket \text {. }
$$

Reasoning parallel to that for L2.I of $N K \alpha$.

Main result: For each line in a derivation let $\mathcal{P}_{i}$ be the expression on line $i$ and $\Gamma_{i}$ be the set of all premises and assumptions whose scope includes line $i$. We set out to show "generalized" soundness: if $\Gamma \vdash_{\mathrm{NCx}}^{*} \mathcal{P}$ then $\Gamma \models_{\mathrm{Cx}_{x}}^{*} \mathcal{P}$. Suppose $\Gamma \vdash_{\mathrm{NCx}}^{*} \mathcal{P}$. Then there is a derivation of $\mathcal{P}$ from premises in $\Gamma$ where $\mathcal{P}$ appears under the scope of the premises alone. By induction on line number of this derivation, we show that for each line $i$ of this derivation, $\Gamma_{i} \models_{C x}^{*} \mathcal{P}_{i}$. The case when $\mathcal{P}_{i}=\mathcal{P}$ is the desired result.

Basis: $\mathcal{P}_{1}$ is a premise or an assumption $A_{s} \llbracket A_{s / t} \rrbracket$. Then $\Gamma_{1}=\left\{A_{s}\right\} \llbracket\left\{A_{s / t}\right\} \rrbracket$; so for any $\left\langle W,\left\{R_{A} \mid A \in \mathfrak{I}\right\}, v\right\rangle_{m}, v_{m}\left(\Gamma_{1}\right)=1$ iff $v_{m(s)}(A)=1 \llbracket m(t) \in$ $f_{A}(m(s)) \rrbracket$; so there is no $\left\langle W,\left\{R_{A} \mid A \in \mathfrak{I}\right\}, v\right\rangle_{m}$ such that $\nu_{m}\left(\Gamma_{1}\right)=1$ but $v_{m(s)}(A)=0 \llbracket m(t) \notin f_{A}(m(s)) \rrbracket$. So by $V_{C x}^{*}, \Gamma_{1} \models_{C x}^{*} A_{s} \llbracket A_{s / t} \rrbracket$, where this is just to say, $\Gamma_{1} \models_{c x}^{*} \mathcal{P}_{1}$.

Assp: For any $i, 1 \leqslant i<k, \Gamma_{i} \models_{C x}^{*} \mathcal{P}_{i}$.

Show: $\Gamma_{k} \models_{C x}^{*} \mathcal{P}_{k}$. 
$\mathcal{P}_{k}$ is either a premise, an assumption, or arises from previous lines by $\mathrm{R}, \supset \mathrm{I}, \supset \mathrm{E}, \wedge \mathrm{I}, \wedge \mathrm{E}, \neg \mathrm{I}, \neg \mathrm{E}, \vee \mathrm{I}, \vee \mathrm{E}, \equiv \mathrm{I}, \equiv \mathrm{E}, \square \mathrm{I} v, \square \mathrm{E} v, \diamond \mathrm{I} v, \diamond \mathrm{E} v$, $>\mathrm{I},>\mathrm{E}$ or, depending on the system, $\mathrm{AMP} \mathrm{P}_{1}, \mathrm{AMP}_{2}, \mathrm{AMs} \mathrm{s}_{1}, \mathrm{AMs}_{2}, \mathrm{AMs}_{3}$, AMRS or AMDL. If $\mathcal{P}_{k}$ is a premise or an assumption, then as in the basis, $\Gamma_{k} \models_{c x}^{*} \mathcal{P}_{k}$. So suppose $\mathcal{P}_{k}$ arises by one of the rules.

(R)

( I)

( $\mathrm{E})$ If $\mathcal{P}_{k}$ arises by $\supset \mathrm{E}$, then the picture is like this,

\begin{tabular}{l|l}
$i$ & $(A \supset B)_{s}$ \\
$j$ & $A_{s}$ \\
$k$ & $B_{s}$
\end{tabular}

where $i, j<k$ and $\mathcal{P}_{k}$ is $B_{s}$. By assumption, $\Gamma_{i} \models_{C x}^{*}(A \supset B)_{s}$ and $\Gamma_{j} \models_{C x}^{*} A_{s}$; but by the nature of access, $\Gamma_{i} \subseteq \Gamma_{k}$ and $\Gamma_{j} \subseteq \Gamma_{k}$; so by L4.I, $\Gamma_{k} \models_{C x}^{*}(A \supset B)_{s}$ and $\Gamma_{k} \models_{C x}^{*} A_{s}$. Suppose $\Gamma_{k} \nvdash_{C x}^{*} B_{s}$; then by VCx* there is some $C x$ interpretation $\left\langle W,\left\{R_{A} \mid A \in \mathfrak{I}\right\}, v\right\rangle_{m}$ such that $v_{m}\left(\Gamma_{k}\right)=1$ but $v_{m(s)}(\mathrm{B})=0$; since $v_{\mathrm{m}}\left(\Gamma_{\mathrm{k}}\right)=1$, by $\mathrm{VCx}^{*}, v_{\mathrm{m}(\mathrm{s})}(\mathrm{A} \supset \mathrm{B})=1$ and $v_{\mathrm{m}(\mathrm{s})}(\mathrm{A})=1$; from the former, by TC $(\supset), v_{\mathrm{m}(\mathrm{s})}(\mathrm{A})=0$ or $v_{\mathrm{m}(\mathrm{s})}(\mathrm{B})=$ 1 ; so $v_{m(s)}(B)=1$. This is impossible; reject the assumption: $\Gamma_{k} \models_{C x}^{*} B_{s}$, which is to say, $\Gamma_{k} \models_{c x}^{*} \mathcal{P}_{k}$.

$(\wedge \mathrm{I})$

$(\wedge \mathrm{E})$

$(\neg \mathrm{I})$ If $\mathcal{P}_{\mathrm{k}}$ arises by $\neg \mathrm{I}$, then the picture is like this,



where $i, j<k$ and $\mathcal{P}_{k}$ is $\neg A_{s}$. By assumption, $\Gamma_{i} \models_{c x}^{*} B_{t}$ and $\Gamma_{j} \models_{c x}^{*} \neg B_{t}$; but by the nature of access, $\Gamma_{\mathrm{i}} \subseteq \Gamma_{\mathrm{k}} \cup\left\{A_{s}\right\}$ and $\Gamma_{\mathrm{j}} \subseteq \Gamma_{\mathrm{k}} \cup\left\{\mathrm{A}_{s}\right\}$; so by L4.I, $\Gamma_{k} \cup\left\{A_{s}\right\} \models_{c x}^{*} B_{t}$ and $\Gamma_{k} \cup\left\{A_{s}\right\} \models_{c x}^{*} \neg B_{t}$. Suppose $\Gamma_{k} \forall_{c x}^{*} \neg A_{s}$; then by VCx* there is a $C x$ interpretation $\left\langle W,\left\{R_{A} \mid A \in \mathfrak{I}\right\}, v\right\rangle_{m}$ such that $v_{\mathrm{m}}\left(\Gamma_{\mathrm{k}}\right)=1$ but $v_{\mathrm{m}(\mathrm{s})}(\neg \mathrm{A})=0$; so by $\mathrm{TC}(\neg), v_{\mathrm{m}(\mathrm{s})}(\mathrm{A})=1$; so $v_{m}\left(\Gamma_{k}\right)=1$ and $v_{m(s)}(A)=1$; so $v_{m}\left(\Gamma_{k} \cup\left\{A_{s}\right\}\right)=1$; so by $V_{C x}^{*}$, $v_{\mathrm{m}(\mathrm{t})}(\mathrm{B})=1$ and $v_{\mathrm{m}(\mathrm{t})}(\neg \mathrm{B})=1$; from the latter, by $\mathrm{TC}(\neg), v_{\mathrm{m}(\mathrm{t})}(\mathrm{B})=$ 0 . This is impossible; reject the assumption: $\Gamma_{k} \models_{C x}^{*} \neg A_{s}$, which is to say, $\Gamma_{k} \models_{c x}^{*} \mathcal{P}_{k}$.

$(\neg \mathrm{E})$ 
( $\square \mathrm{I} v$ ) If $\mathcal{P}_{k}$ arises by $\square \mathrm{I} v$, then the picture is like this,

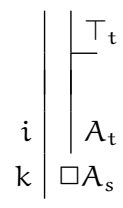

where $i<k$, $t$ does not appear in any member of $\Gamma_{k}$ (in any undischarged premise or assumption), and $\mathcal{P}_{k}$ is $\square A_{s}$. By assumption, $\Gamma_{i} \models_{C_{x}}^{*} A_{t}$; but by the nature of access, $\Gamma_{i} \subseteq \Gamma_{k} \cup\left\{T_{t}\right\}$; so by L4.I, $\Gamma_{k} \cup\left\{T_{t}\right\} \models_{C x}^{*}$ $A_{t}$. Suppose $\Gamma_{k} \forall_{C x}^{*} \square A_{s}$; then by $\mathrm{VCx}^{*}$, there is a $C x$ interpretation $\left\langle W,\left\{R_{A} \mid A \in \mathfrak{I}\right\}, v\right\rangle_{m}$ such that $v_{m}\left(\Gamma_{k}\right)=1$ but $v_{m(s)}(\square A)=0$; so by $\mathrm{TC}(\square)_{v}$, there is some $w \in W$ such that $v_{w}(A)=0$. Now consider a map $m^{\prime}$ like $m$ except that $m^{\prime}(t)=w$, and consider $\left\langle W,\left\{R_{A} \mid A \in\right.\right.$ $\left.\left.I_{\}}\right\}, v\right\rangle_{m^{\prime}}$; since $t$ does not appear in $\Gamma_{k}$, it remains that $v_{m^{\prime}}\left(\Gamma_{k}\right)=1$; and, at any world, $v_{m^{\prime}(t)}(T)=1$; so $v_{m^{\prime}}\left(\Gamma_{k} \cup\left\{T_{t}\right\}\right)=1$; so by $V_{C x}^{*}$, $v_{m^{\prime}(t)}(A)=1$. But $m^{\prime}(t)=w$; so $v_{w}(A)=1$. This is impossible; reject the assumption: $\Gamma_{k} \models_{C x}^{*} \square A_{s}$, which is to say, $\Gamma_{k} \models_{C x}^{*} \mathcal{P}_{k}$.

( $\square \mathrm{Ev}$ ) If $\mathcal{P}_{k}$ arises by $\square \mathrm{Ev}$, then the picture is like this,

$$
\begin{array}{l|l}
i & \square A_{s} \\
k & A_{t}
\end{array}
$$

where $i<k$ and $\mathcal{P}_{k}$ is $A_{t}$. By assumption, $\Gamma_{i} \models_{C x}^{*} \square A_{s}$; but by the nature of access, $\Gamma_{\mathrm{i}} \subseteq \Gamma_{\mathrm{k}}$; so by L4.I, $\Gamma_{\mathrm{k}} \models_{c x}^{*} \square A_{s}$. Suppose $\Gamma_{k} \not \nvdash_{c x}^{*} A_{t}$; then by $\mathrm{VCx}^{*}$, there is some $C x$ interpretation $\left\langle\mathrm{W},\left\{\mathrm{R}_{\mathrm{A}} \mid \mathrm{A} \in \mathfrak{I}\right\}, v\right\rangle_{\mathrm{m}}$ such that $v_{\mathrm{m}}\left(\Gamma_{\mathrm{k}}\right)=1$ but $v_{\mathrm{m}(\mathrm{t})}(A)=0$; since $v_{\mathrm{m}}\left(\Gamma_{\mathrm{k}}\right)=1$, by $\mathrm{VCx}^{*}$, $v_{\mathrm{m}(\mathrm{s})}(\square A)=1$; so by $\mathrm{TC}(\square)_{v}$, any $w$ has $v_{w}(A)=1$; so $v_{\mathrm{m}(\mathrm{t})}(A)=1$. This is impossible; reject the assumption: $\Gamma_{k} \models_{C x}^{*} A_{t}$, which is to say, $\Gamma_{\mathrm{k}} \models_{C x}^{*} \mathcal{P}_{\mathrm{k}}$.

$(\diamond \mathrm{I} v)$

$(\diamond \mathrm{Ev})$ If $\mathcal{P}_{k}$ arises by $\diamond \mathrm{Ev}$, then the picture is like this,

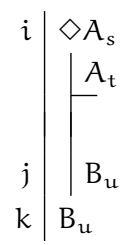


where $i, j<k$, $t$ does not appear in any member of $\Gamma_{k}$ (in any undischarged premise or assumption) and is not $u$, and $\mathcal{P}_{k}$ is $B_{u}$. By assumption, $\Gamma_{i} \models_{C x}^{*} \diamond A_{s}$ and $\Gamma_{j} \models_{C x}^{*} B_{\mathfrak{u}}$; but by the nature of access, $\Gamma_{i} \subseteq \Gamma_{k}$ and $\Gamma_{j} \subseteq \Gamma_{k} \cup\left\{A_{t}\right\}$; so by L4.I, $\Gamma_{k} \models_{C x}^{*} \diamond A_{s}$ and $\Gamma_{k} \cup\left\{A_{t}\right\} \models_{C x}^{*} B_{u}$. Suppose $\Gamma_{k} \forall_{C x}^{*} B_{\mathcal{u}}$; then by $V_{C x}^{*}$, there is a $C x$ interpretation $\left\langle W,\left\{R_{A} \mid A \in\right.\right.$ $\Im\}, v\rangle_{\mathfrak{m}}$ such that $v_{\mathfrak{m}}\left(\Gamma_{k}\right)=1$ but $v_{\mathfrak{m}(\mathfrak{u})}(\mathrm{B})=0$. Since $v_{\mathrm{m}}\left(\Gamma_{\mathrm{k}}\right)=1$, by $\mathrm{VCx}^{*}, v_{\mathrm{m}(s)}(\diamond \mathrm{A})=1$; so by $\mathrm{TC}(\diamond)_{v}$, there is some $w \in W$ such that $v_{w}(A)=1$. Now consider a map $m^{\prime}$ like $m$ except that $m^{\prime}(t)=w$, and consider $\left\langle W,\left\{R_{A} \mid A \in \mathfrak{I}\right\}, v\right\rangle_{m^{\prime}}$; since $t$ does not appear in $\Gamma_{k}$, it remains that $v_{m^{\prime}}\left(\Gamma_{k}\right)=1$; and since $m^{\prime}(t)=w, v_{m^{\prime}(t)}(A)=1$; so $v_{m^{\prime}}\left(\Gamma_{k} \cup\left\{A_{t}\right\}\right)=1$; so by $\mathrm{VCx}^{*}, v_{m^{\prime}(u)}(B)=1$. But since $t \neq u$, $\mathrm{m}^{\prime}(\mathfrak{u})=\mathfrak{m}(\mathfrak{u})$; so $v_{\mathfrak{m}(u)}(B)=1$. This is impossible; reject the assumption: $\Gamma_{k} \models_{c x}^{*} B_{u}$, which is to say, $\Gamma_{k} \models_{c x}^{*} \mathcal{P}_{k}$.

( $>$ I) If $\mathcal{P}_{k}$ arises by $>\mathrm{I}$, then the picture is like this,

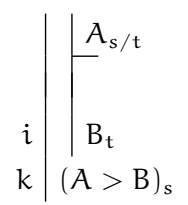

where $i<k$, $t$ does not appear in any member of $\Gamma_{k}$ (in any undischarged premise or assumption), and $\mathcal{P}_{k}$ is $(A>B)_{s}$. By assumption, $\Gamma_{i} \models_{C x}^{*} B_{t}$; but by the nature of access, $\Gamma_{i} \subseteq \Gamma_{k} \cup\left\{A_{s / t}\right\}$; so by L4.I, $\Gamma_{k} \cup\left\{A_{s / t}\right\} \models_{C x}^{*} B_{t}$. Suppose $\Gamma_{k} \not \models_{C x}^{*}(A>B)_{s}$; then by $V C^{*}$, there is a $C x$ interpretation $\left\langle W,\left\{R_{A} \mid A \in \mathfrak{I}\right\}, v\right\rangle_{m}$ such that $v_{m}\left(\Gamma_{k}\right)=1$ but $v_{\mathrm{m}(s)}(\mathrm{A}>\mathrm{B})=0$; so by $\mathrm{TC}(>)$, there is some $w \in W$ such that $m(s) R_{A} w$ but $v_{w}(B)=0$. Now consider a map $m^{\prime}$ like $m$ except that $\mathrm{m}^{\prime}(\mathrm{t})=w$, and consider $\left\langle\mathrm{W},\left\{\mathrm{R}_{\mathrm{A}} \mid \mathrm{A} \in \mathfrak{I}\right\}, v\right\rangle_{\mathrm{m}^{\prime}}$; since $t$ does not appear in $\Gamma_{k}$, it remains that $v_{m^{\prime}}\left(\Gamma_{k}\right)=1$; and since $m^{\prime}(t)=w$ and $m^{\prime}(s)=m(s)$, $\left\langle\mathrm{m}^{\prime}(\mathrm{s}), \mathrm{m}^{\prime}(\mathrm{t})\right\rangle \in \mathrm{R}_{\mathrm{A}}$; so $v_{\mathrm{m}^{\prime}}\left(\Gamma_{\mathrm{k}} \cup\left\{\mathrm{A}_{\mathrm{s} / \mathrm{t}}\right\}\right)=1$; so by $\mathrm{VCx}^{*}, v_{\mathrm{m}^{\prime}(\mathrm{t})}(\mathrm{B})=1$. But $\mathrm{m}^{\prime}(\mathrm{t})=w$; so $v_{w}(\mathrm{~B})=1$. This is impossible; reject the assumption: $\Gamma_{k} \models_{C x}^{*}(A>B)_{s}$, which is to say, $\Gamma_{k} \models_{C x}^{*} \mathcal{P}_{k}$.

(>E) If $\mathcal{P}_{k}$ arises by $>\mathrm{E}$, then the picture is like this,

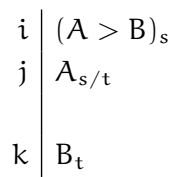

where $i, j<k$ and $\mathcal{P}_{k}$ is $B_{t}$. By assumption, $\Gamma_{i} \models_{C_{x}}^{*}(A>B)_{s}$ and $\Gamma_{j} \models_{C_{x}}^{*}$ $A_{s / t}$; but by the nature of access, $\Gamma_{\mathrm{i}} \subseteq \Gamma_{\mathrm{k}}$ and $\Gamma_{\mathrm{j}} \subseteq \Gamma_{\mathrm{k}}$; so by L4.I, $\Gamma_{\mathrm{k}} \models_{C x}^{*}$ $(A>B)_{s}$ and $\Gamma_{k} \models_{C x}^{*} A_{s / t}$. Suppose $\Gamma_{k} \forall_{C x}^{*} B_{t}$; then by VCx ${ }^{*}$, there is some $C x$ interpretation $\left\langle W,\left\{R_{A} \mid A \in \mathfrak{I}\right\}, v\right\rangle_{m}$ such that $v_{m}\left(\Gamma_{k}\right)=1$ but $v_{\mathrm{m}(\mathrm{t})}(\mathrm{B})=0$; since $v_{\mathrm{m}}\left(\Gamma_{\mathrm{k}}\right)=1$, by $\mathrm{VCx}^{*}, v_{\mathrm{m}(\mathrm{s})}(\mathrm{A}>\mathrm{B})=1$ and $\langle\mathrm{m}(\mathrm{s}), \mathrm{m}(\mathrm{t})\rangle \in \mathrm{R}_{\mathrm{A}}$; from the former, by $\mathrm{TC}(>)$, any $w \in W$ such that 
$m(s) R_{A} w$ has $v_{w}(B)=1$; so $v_{m(t)}(B)=1$. This is impossible; reject the assumption: $\Gamma_{k} \models_{C x}^{*} B_{t}$, which is to say, $\Gamma_{k} \models_{C x}^{*} \mathcal{P}_{k}$.

(AMPI) If $\mathcal{P}_{k}$ arises by $A M P_{I}$, then the picture is like this,

$$
\begin{array}{l|l}
i & A_{s / t} \\
k & A_{t}
\end{array}
$$

where $i<k$ and $\mathcal{P}_{k}$ is $A_{t}$. Where this rule is in $N C x, C x$ includes condition (I). By assumption, $\Gamma_{\mathrm{i}} \models_{C x}^{*} A_{s / t}$; but by the nature of access, $\Gamma_{\mathrm{i}} \subseteq \Gamma_{\mathrm{k}}$; so by L4.I, $\Gamma_{\mathrm{k}} \models_{C_{x}}^{*} A_{s / \mathrm{t}}$. Suppose $\Gamma_{\mathrm{k}} \forall_{c x}^{*} A_{\mathrm{t}}$; then by VCx*, there is some $C x$ interpretation $\left\langle\mathrm{W},\left\{\mathrm{R}_{\mathrm{A}} \mid A \in \mathfrak{I}\right\}, v\right\rangle_{\mathrm{m}}$ such that $v_{\mathrm{m}}\left(\Gamma_{\mathrm{k}}\right)=$ 1 but $v_{m(t)}(A)=0$; since $v_{m}\left(\Gamma_{k}\right)=1$, by VCx ${ }^{*}, m(t) \in f_{A}(m(s))$; so by condition $(I), m(t) \in[A]$; so $v_{m(t)}(A)=1$. This is impossible; reject the assumption: $\Gamma_{k} \models_{C x}^{*} A_{t}$, which is to say, $\Gamma_{k} \models_{C x}^{*} \mathcal{P}_{k}$.

$\left(\mathrm{AMP}_{2}\right)$ If $\mathcal{P}_{k}$ arises by $\mathrm{AMP}_{2}$, then the picture is like this,

$$
\begin{array}{l|l}
i & A_{t} \\
k & A_{t / t}
\end{array}
$$

where $i<k$ and $\mathcal{P}_{k}$ is $A_{t / t}$. Where this rule is in $N C x, C x$ includes condition (2). By assumption, $\Gamma_{i} \models_{c x}^{*} A_{t}$; but by the nature of access, $\Gamma_{\mathrm{i}} \subseteq \Gamma_{\mathrm{k}}$; so by $\mathrm{L}_{4} \cdot \mathrm{I}, \Gamma_{\mathrm{k}} \models_{c x}^{*} A_{\mathrm{t}}$. Suppose $\Gamma_{\mathrm{k}} \not \forall_{\mathrm{Cx}}^{*} A_{\mathrm{t} / \mathrm{t}}$; then by VCx${ }^{*}$, there is some $C x$ interpretation $\left\langle W,\left\{R_{A} \mid A \in I\right\}, v\right\rangle_{m}$ such that $v_{m}\left(\Gamma_{k}\right)=$ 1 but $m(t) \notin f_{A}(m(t))$; since $v_{m}\left(\Gamma_{k}\right)=1$, by $V_{C x}^{*}, v_{m}(t)(A)=1$; so $m(t) \in[A]$; so by condition $(2), m(t) \in f_{A}(m(t))$. This is impossible; reject the assumption: $\Gamma_{k} \models_{c x}^{*} A_{t / t}$, which is to say, $\Gamma_{k} \models_{c x}^{*} \mathcal{P}_{k}$.

$\left(\mathrm{AMs}_{\mathrm{I}}\right)$ If $\mathcal{P}_{k}$ arises by $\mathrm{AMs}$, then the picture is like this,



where $i, j<k$, $t$ does not appear in any member of $\Gamma_{k}$ (in any undischarged premise or assumption) and is not $u$, and $\mathcal{P}_{k}$ is $B_{\mathfrak{u}}$. Where this rule is in NCx,Cx includes condition (3). By assumption, $\Gamma_{i} \models_{C x}^{*} \diamond A_{s}$ and $\Gamma_{j} \models_{c x}^{*} B_{u}$; but by the nature of access, $\Gamma_{i} \subseteq \Gamma_{k}$ and $\Gamma_{j} \subseteq \Gamma_{k} \cup\left\{A_{s / t}\right\}$; so by $\mathrm{L}_{4} \cdot \mathrm{I}, \Gamma_{\mathrm{k}} \models_{c x}^{*} \diamond A_{s}$ and $\Gamma_{k} \cup\left\{A_{s / t}\right\} \models_{c x}^{*} B_{u}$. Suppose $\Gamma_{k} \not \nvdash_{c x}^{*} B_{u}$; then by $\mathrm{VCx}^{*}$, there is a $C x$ interpretation $\left\langle W,\left\{\mathrm{R}_{\mathrm{A}} \mid A \in \mathfrak{I}\right\}, v\right\rangle_{\mathrm{m}}$ such that $v_{\mathrm{m}}\left(\Gamma_{\mathrm{k}}\right)=1$ but $v_{\mathrm{m}(\mathrm{u})}(\mathrm{B})=0$. Since $v_{\mathrm{m}}\left(\Gamma_{\mathrm{k}}\right)=1$, by $\mathrm{VCx}^{*}$, $v_{\mathrm{m}(\mathrm{s})}(\diamond \mathrm{A})=1$; so by $\mathrm{TC}(\diamond)_{v}$, there is some $w \in W$ such that $v_{w}(A)=$ 1 ; so $w \in[A]$ and $[A] \neq \phi$; so by condition $(3), f_{A}(m(s)) \neq \phi$; so there 
is some $x \in f_{A}(m(s))$. Now consider a map $m^{\prime}$ like $m$ except that $\mathrm{m}^{\prime}(\mathrm{t})=\mathrm{x}$, and consider $\left\langle\mathrm{W},\left\{\mathrm{R}_{\mathrm{A}} \mid \mathrm{A} \in \mathfrak{I}\right\}, v\right\rangle_{\mathrm{m}^{\prime}}$; since $\mathrm{t}$ does not appear in $\Gamma_{k}$, it remains that $v_{m^{\prime}}\left(\Gamma_{k}\right)=1$; and since $m^{\prime}(t)=x$ and $m^{\prime}(s)=$ $m(s), m^{\prime}(t) \in f_{A}\left(m^{\prime}(s)\right)$; so $v_{m^{\prime}}\left(\Gamma_{k}\right)=1$ and $\left\langle m^{\prime}(s), m^{\prime}(t)\right\rangle \in R_{A}$; so $v_{\mathfrak{m}^{\prime}}\left(\Gamma_{\mathrm{k}} \cup\left\{\mathrm{A}_{\mathrm{s} / \mathrm{t}}\right\}\right)=1$; so by $\mathrm{VCx}^{*}, v_{\mathrm{m}^{\prime}(\mathfrak{u})}(\mathrm{B})=1$. But since $\mathrm{t} \neq \mathrm{u}$, $\mathrm{m}^{\prime}(\mathrm{u})=\mathrm{m}(\mathrm{u})$; so $v_{\mathfrak{m}(\mathfrak{u})}(B)=1$. This is impossible; reject the assumption: $\Gamma_{k} \models_{c x}^{*} B_{\mathfrak{u}}$, which is to say, $\Gamma_{k} \models_{c x}^{*} \mathcal{P}_{k}$.

$\left(\mathrm{AMs}_{2}\right)$ If $\mathcal{P}_{\mathrm{k}}$ arises by $\mathrm{AMs}_{2}$, then the picture is like this,

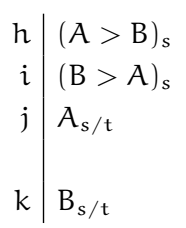

where $h, i, j<k$ and $\mathcal{P}_{k}$ is $B_{s / t}$. Where this rule is in $N C x, C x$ includes condition (4). By assumption, $\Gamma_{h} \models_{C x}^{*}(A>B)_{s}, \Gamma_{i} \models_{C x}^{*}(B>A)_{s}$ and $\Gamma_{j} \models_{c x}^{*} A_{s / t}$; but by the nature of access, $\Gamma_{h} \subseteq \Gamma_{k}, \Gamma_{i} \subseteq \Gamma_{k}$ and $\Gamma_{j} \subseteq$ $\Gamma_{k}$; so by L4.I, $\Gamma_{k} \models_{C x}^{*}(A>B)_{s}, \Gamma_{k} \models_{C x}^{*}(B>A)_{s}$, and $\Gamma_{k} \models_{C x}^{*} A_{s / t}$. Suppose $\Gamma_{\mathrm{k}} \forall_{C x}^{*} \mathrm{~B}_{\mathrm{s} / \mathrm{t}}$; then by $\mathrm{VCx}^{*}$, there is some $C x$ interpretation $\left\langle W,\left\{R_{A} \mid A \in \mathfrak{I}\right\}, v\right\rangle_{m}$ such that $v_{m}\left(\Gamma_{k}\right)=1$ but $m(t) \notin f_{B}(m(s))$; since $v_{m}\left(\Gamma_{k}\right)=1$, by VCx ${ }^{*}, v_{m(s)}(A>B)=1, v_{m(s)}(B>A)=1$; and $m(t) \in$ $f_{A}(m(s))$. Suppose $w \in f_{A}(m(s))$; then $m(s) R_{A} w$ and since $v_{m(s)}(A>$ $\mathrm{B})=1$, by $\mathrm{TC}(>), v_{w}(\mathrm{~B})=1$; so $w \in[\mathrm{B}]$ and, generalizing, we have that $f_{A}(m(s)) \subseteq[B]$. Suppose $w \in f_{B}(m(s))$; then $m(s) R_{B} w$ and since $v_{m(s)}(B>A)=1$, by TC $(>), v_{w}(A)=1$; so $w \in[A]$ and, generalizing, we have that $f_{B}(m(s)) \subseteq[A]$. So $f_{A}(m(s)) \subseteq[B]$ and $f_{B}(m(s)) \subseteq[A]$; so by condition (4), $f_{A}(m(s))=f_{B}(m(s))$; thus since $m(t) \in f_{A}(m(s))$, $m(t) \in f_{B}(m(s))$. This is impossible; reject the assumption: $\Gamma_{k} \models_{C x}^{*}$ $\mathrm{B}_{\mathrm{s} / \mathrm{t}}$, which is to say, $\Gamma_{\mathrm{k}} \models_{\mathrm{Cx}}^{*} \mathcal{P}_{\mathrm{k}}$.

$\left(\mathrm{AMs}_{3}\right)$ If $\mathcal{P}_{\mathrm{k}}$ arises by $\mathrm{AMs}_{3}$, then the picture is like this,

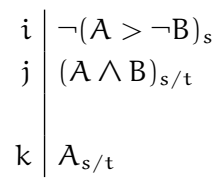

where $i, j<k$ and $\mathcal{P}_{k}$ is $A_{s / t}$. Where this rule is in $N C x, C x$ includes condition (5). By assumption, $\Gamma_{i} \models_{C x}^{*} \neg(A>\neg B)_{s}$ and $\Gamma_{j} \models_{C x}^{*}(A \wedge B)_{s / t}$; but by the nature of access, $\Gamma_{\mathrm{i}} \subseteq \Gamma_{\mathrm{k}}$ and $\Gamma_{\mathrm{j}} \subseteq \Gamma_{\mathrm{k}}$; so by L4.I, $\Gamma_{\mathrm{k}} \models_{C_{x}}^{*}$ $\neg(A>\neg B)_{s}$, and $\Gamma_{k} \models_{C x}^{*}(A \wedge B)_{s / t}$. Suppose $\Gamma_{k} \forall_{C x}^{*} A_{s / t}$; then by $\mathrm{VCx}^{*}$, there is some $C x$ interpretation $\left\langle\mathrm{W},\left\{\mathrm{R}_{\mathrm{A}} \mid A \in \mathfrak{I}\right\}, v\right\rangle_{\mathrm{m}}$ such that $v_{m}\left(\Gamma_{k}\right)=1$ but $m(t) \notin f_{A}(m(s))$; since $v_{m}\left(\Gamma_{k}\right)=1$, by $V_{C x} *$, $v_{m(s)}(\neg(A>\neg B))=1$, and $m(t) \in f_{A \wedge B}(m(s))$. Since $v_{m(s)}(\neg(A>$ $\neg \mathrm{B}))=1$, by $\mathrm{TC}(\neg), v_{\mathrm{m}(s)}(\mathrm{A}>\neg \mathrm{B})=0$; so by $\mathrm{TC}(>)$, there is some 
$w \in W$ such that $m(s) R_{A} w$ and $v_{w}(\neg \mathrm{B})=0$; so by $\mathrm{TC}(\neg), v_{w}(\mathrm{~B})=$ 1 ; but $w \in \mathrm{f}_{A}(\mathrm{~m}(\mathrm{~s}))$; so $\mathrm{f}_{A}(\mathrm{~m}(\mathrm{~s})) \cap[B] \neq \phi$; so by condition (5), $f_{A \wedge B}(m(s)) \subseteq f_{A}(m(s)) ;$ so $m(t) \in f_{A}(m(s))$. This is impossible; reject the assumption: $\Gamma_{k} \models_{C x}^{*} A_{s / t}$, which is to say, $\Gamma_{k} \models_{C x}^{*} \mathcal{P}_{k}$.

(AMrs) If $\mathcal{P}_{k}$ arises by AMrs, then the picture is like this,

$$
\begin{array}{r|l}
\mathrm{h} & A_{s / \mathrm{t}} \\
\mathrm{i} & A_{s / \mathrm{u}} \\
\mathrm{j} & \mathcal{Q}_{(\mathrm{t})} \\
\mathrm{k} & \mathbf{Q}_{(\mathrm{u})}
\end{array}
$$

where $h, i, j<k$ and $\mathcal{P}_{k}$ is $Q_{(u)}$. Suppose $Q_{(t)}$ is some $B_{t}$ and $Q_{(u)}$ is $\mathrm{B}_{\mathrm{u}}$. Where this rule is in NCx,Cx includes condition (6). By assumption, $\Gamma_{h} \models_{c x}^{*} A_{s / t}, \Gamma_{i} \models_{C x}^{*} A_{s / u}$ and $\Gamma_{j} \models_{c x}^{*} B_{t}$; but by the nature of access, $\Gamma_{h} \subseteq \Gamma_{k}, \Gamma_{i} \subseteq \Gamma_{k}$ and $\Gamma_{j} \subseteq \Gamma_{k}$; so by L4.I, $\Gamma_{k} \models_{c x}^{*} A_{s / t}$, $\Gamma_{k} \models_{c x}^{*} A_{s / u}$, and $\Gamma_{k} \models_{c x}^{*} B_{t}$. Suppose $\Gamma_{k} \not \models_{c x}^{*} B_{u}$; then by VCx* there is some $C x$ interpretation $\left\langle W,\left\{R_{A} \mid A \in \mathfrak{I}\right\}, v\right\rangle_{m}$ such that $v_{m}\left(\Gamma_{k}\right)=1$ but $v_{\mathrm{m}(\mathrm{u})}(\mathrm{B})=0$; since $v_{\mathrm{m}}\left(\Gamma_{\mathrm{k}}\right)=1$, by $\mathrm{VCx}^{*}, \mathrm{~m}(\mathrm{t}) \in \mathrm{f}_{\mathrm{A}}(\mathrm{m}(\mathrm{s}))$, $m(u) \in f_{A}(m(s))$, and $v_{m(t)}(B)=1$. With the first two of these, by condition (6), $m(t)=m(u)$; so $v_{m(u)}(B)=1$. This is impossible; reject the assumption: $\Gamma_{k} \models_{C_{x}}^{*} B_{u}$, which is to say, $\Gamma_{k} \models_{C_{x}}^{*} \mathcal{P}_{k}$. And similarly when $Q_{(t)}$ is $B_{t / v}, B_{v / t}$, or $B_{t / t}$.

(AMDL) If $\mathcal{P}_{k}$ arises by AMDL, then the picture is like this,
\begin{tabular}{r|l}
$h$ & $A_{s}$ \\
$i$ & $A_{s / t}$ \\
$j$ & $Q_{(t)}$ \\
$k$ & $Q_{(s)}$
\end{tabular}

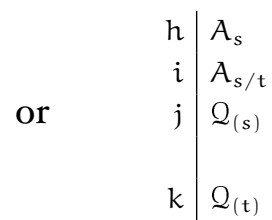

where $h, i, j<k$ and, in the left-hand case, $\mathcal{P}_{k}$ is $\mathcal{Q}_{(s)}$. Suppose $\mathcal{Q}_{(t)}$ is of the sort $\mathrm{B}_{\mathrm{t} / v}$ and $Q_{(s)}$ is $\mathrm{B}_{s / v}$. Where this rule is in $N C x, C x$ includes condition (7). By assumption, $\Gamma_{h} \models_{c x}^{*} A_{s}, \Gamma_{i} \models_{c x}^{*} A_{s / t}$ and $\Gamma_{j} \models_{c x}^{*} B_{t / v}$; but by the nature of access, $\Gamma_{\mathrm{h}} \subseteq \Gamma_{\mathrm{k}}, \Gamma_{\mathrm{i}} \subseteq \Gamma_{\mathrm{k}}$ and $\Gamma_{\mathrm{j}} \subseteq \Gamma_{\mathrm{k}}$; so by L4.I, $\Gamma_{k} \models_{c x}^{*} A_{s}, \Gamma_{k} \models_{c x}^{*} A_{s / t}$, and $\Gamma_{k} \models_{c x}^{*} B_{t / v}$. Suppose $\Gamma_{k} \not \models_{c x}^{*} B_{s / v}$; then by $\mathrm{VCx}^{*}$, there is some $C x$ interpretation $\left\langle\mathrm{W},\left\{\mathrm{R}_{\mathrm{A}} \mid A \in \mathfrak{I}\right\}, v\right\rangle_{\mathrm{m}}$ such that $v_{\mathrm{m}}\left(\Gamma_{\mathrm{k}}\right)=1$ but $\langle\mathrm{m}(\mathrm{s}), \mathrm{m}(v)\rangle \notin \mathrm{R}_{\mathrm{B}}$; since $v_{\mathrm{m}}\left(\Gamma_{\mathrm{k}}\right)=1$, by $\mathrm{VCx}^{*}$, $v_{m(s)}(A)=1, m(t) \in f_{A}(m(s))$, and $\langle m(t), m(v)\rangle \in R_{B}$. From the first of these, $m(s) \in[A]$; so by condition $(7), m(s)=m(t)$; so $\langle m(s), m(v)\rangle \in$ $R_{B}$. This is impossible; reject the assumption: $\Gamma_{k} \models_{C x}^{*} B_{s / v}$ which is to say, $\Gamma_{k} \models_{C x}^{*} \mathcal{P}_{k}$. And similarly when $\mathcal{Q}_{(t)}$ is $B_{t}, B_{v / t}$ or $B_{t / t}$. And similarly in the right-hand case.

For any $i, \Gamma_{i} \models_{C x}^{*} \mathcal{P}_{i}$. 
THEOREM 4.2 NCx is complete: if $\Gamma \models_{C x}$ A then $\Gamma \vdash_{N C x}$ A.

Suppose $\Gamma \models_{C x} A$; then $\Gamma_{0} \models_{C x}^{*} A_{0}$; we show that $\Gamma_{0} \vdash_{N C x}^{*} A_{0}$. Again, this reduces to the standard notion. For the following, fix on some particular constraint(s) $x$. Then definitions of consistency etc. are relative to it.

Con $\Gamma$ is CONSISTENT iff there is no $A_{s}$ such that $\Gamma \vdash_{\mathrm{NCx}}^{*} A_{s}$ and $\Gamma \vdash_{\mathrm{NCx}}^{*} \neg A_{s}$.

$\mathrm{L}_{4.2}$ If $\mathrm{s}$ is 0 or appears in $\Gamma$, and $\Gamma \nvdash_{\mathrm{NCx}}^{*} \neg \mathrm{P}_{\mathrm{s}}$, then $\Gamma \cup\left\{\mathrm{P}_{\mathrm{s}}\right\}$ is consistent.

Reasoning parallel to $\mathrm{L}_{2} .2$ for $N K \alpha$.

L4.3 There is an enumeration of all the subscripted formulas, $\mathcal{P}_{1} \mathcal{P}_{2} \ldots$

Proof by construction as for $\mathrm{L} 2.3$ for $N K \alpha$.

Max $\Gamma$ is s-MAXImaL iff for any $A_{s}$ either $\Gamma \vdash_{\mathrm{NCx}}^{*} A_{s}$ or $\Gamma \vdash_{\mathrm{NCx}}^{*} \neg A_{s}$.

SGT $\Gamma$ is a sCAPEgOAT set for $\square$ iff for every formula of the form $\neg \square A_{s}$, if $\Gamma \vdash_{\mathrm{NCx}}^{*} \neg \square A_{\mathrm{s}}$ then there is some $\mathrm{t}$ such that $\Gamma \vdash_{\mathrm{NCx}}^{*} \neg A_{t}$.

$\Gamma$ is a SCAPEGOAT set for $>$ iff for any formula of the form $\neg(A>B)_{s}$, if $\Gamma \vdash_{\mathrm{NCx}}^{*} \neg(A>B)_{s}$ then there is some $t$ such that $\Gamma \vdash_{\mathrm{NCx}}^{*} A_{s / t}$ and $\Gamma \vdash_{\mathrm{NCx}}^{*} \neg \mathrm{B}_{\mathrm{t}}$.

$\mathrm{C}\left(\Gamma^{\prime}\right)$ For $\Gamma$ with unsubscripted formulas and the corresponding $\Gamma_{0}$, we construct $\Gamma^{\prime}$ as follows. Set $\Omega_{0}=\Gamma_{0}$. By L $4 \cdot 3$, there is an enumeration, $\mathcal{P}_{1}, \mathcal{P}_{2} \ldots$ of all the subscripted formulas; let $\mathcal{E}_{0}$ be this enumeration. Then for the first $A_{s}$ in $\mathcal{E}_{i-1}$ such that $s$ is 0 or included in $\Omega_{i-1}$, let $\varepsilon_{i}$ be like $\varepsilon_{i-1}$ but without $A_{s}$, and set,

and

$$
\begin{array}{ll}
\Omega_{i}=\Omega_{i-1} & \text { if } \Omega_{i-1} \vdash_{N C x}^{*} \neg A_{s} \\
\Omega_{i^{*}}=\Omega_{i-1} \cup\left\{A_{s}\right\} & \text { if } \Omega_{i-1} \nvdash_{N C x}^{*} \neg A_{s}
\end{array}
$$

$$
\begin{array}{ll}
\Omega_{i}=\Omega_{\mathfrak{i}^{*}} & \text { if } A_{s} \text { is not of the form } \neg \square P_{s} \text { or } \neg(P>Q)_{s} \\
\Omega_{i}=\Omega_{\mathfrak{i}^{*}} \cup\left\{\neg P_{t}\right\} & \text { if } A_{s} \text { is of the form } \neg \square P_{s} \\
\Omega_{i}=\Omega_{\mathfrak{i}^{*}} \cup\left\{P_{s / t}, \neg Q_{t}\right\} & \text { if } A_{s} \text { is of the form } \neg(P>Q)_{s} \\
\text {-where } t \text { is the first subscript not included in } \Omega_{\mathfrak{i}^{*}}
\end{array}
$$

then

$$
\Gamma^{\prime}=\bigcup_{i \geqslant 0} \Omega_{i}
$$

Note that there is always sure to be a subscript $t$ not in $\Omega_{i^{*}}$ insofar as there are infinitely many subscripts, and at any stage only finitely many formulas are added - the only subscripts in the initial $\Omega_{0}$ being 0 . Suppose $s$ is introduced in $\Gamma^{\prime}$; then there is some $\Omega_{i}$ in which it is first introduced; and any formula $\mathcal{P}_{j}$ in the original enumeration that has subscript $s$ is sure to be "considered" for inclusion at a subsequent stage.

L4.4 For any s included in $\Gamma^{\prime}, \Gamma^{\prime}$ is s-maximal.

Reasoning parallel to $\mathrm{L}_{2} .4$ for $N K \alpha$. 
$\mathrm{L}_{4} .5$ If $\Gamma_{0}$ is consistent, then each $\Omega_{i}$ is consistent.

Suppose $\Gamma_{0}$ is consistent.

Basis: $\Omega_{0}=\Gamma_{0}$ and $\Gamma_{0}$ is consistent; so $\Omega_{0}$ is consistent.

Assp: For any $i, 0 \leqslant i<k, \Omega_{i}$ is consistent.

Show: $\Omega_{k}$ is consistent.

$\Omega_{\mathrm{k}}$ is either (i) $\Omega_{\mathrm{k}-1}$, (ii) $\Omega_{\mathrm{k}^{*}}=\Omega_{\mathrm{k}-1} \cup\left\{\mathrm{A}_{\mathrm{s}}\right\}$, (iii) $\Omega_{\mathrm{k}^{*}} \cup\left\{\neg \mathrm{P}_{\mathrm{t}}\right\}$ or (iv) $\Omega_{k^{*}} \cup\left\{P_{s / t}, \neg Q_{t}\right\}$.

(i) Suppose $\Omega_{k}$ is $\Omega_{k-1}$. By assumption, $\Omega_{k-1}$ is consistent; so $\Omega_{k}$ is consistent.

(ii) Suppose $\Omega_{k}$ is $\Omega_{k^{*}}=\Omega_{k-1} \cup\left\{A_{s}\right\}$. Then by construction, $s$ is 0 or in $\Omega_{k-1}$ and $\Omega_{k-1} \nvdash_{N C x}^{*} \neg A_{s}$; so by $L_{4.2}, \Omega_{k-1} \cup\left\{A_{s}\right\}$ is consistent; so $\Omega_{k}$ is consistent.

(iii) Suppose $\Omega_{k}$ is $\Omega_{k^{*}} \cup\left\{\neg P_{t}\right\}$. In this case, as above, $\Omega_{k *}$ is consistent and by construction, $\neg \square P_{s} \in \Omega_{k^{*}}$. Suppose $\Omega_{k}$ is inconsistent. Then there are $A_{u}$ and $\neg A_{u}$ such that $\Omega_{k^{*}} \cup\left\{\neg P_{t}\right\} \vdash_{\text {NCx }}^{*} A_{u}$ and $\Omega_{\mathrm{k}^{*}} \cup\left\{\neg \mathrm{P}_{\mathrm{t}}\right\} \vdash_{\mathrm{NCx}}^{*} \neg A_{\mathrm{u}}$. So reason as follows,

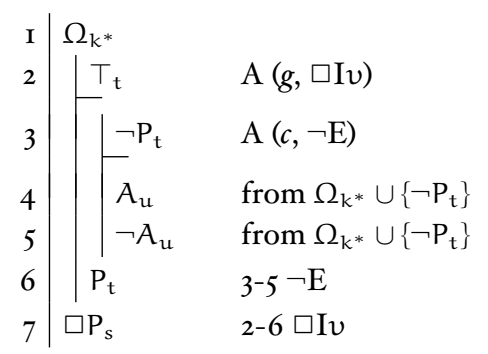

where, by construction, $t$ is not in $\Omega_{\mathrm{k}^{*}}$. So $\Omega_{\mathrm{k}^{*}} \vdash_{\mathrm{NCx}}^{*} \square \mathrm{P}_{s}$; but $\neg \square \mathrm{P}_{\mathrm{s}} \in \Omega_{\mathrm{k}^{*}}$; so $\Omega_{\mathrm{k}^{*}} \vdash_{\mathrm{NCx}}^{*} \neg \square \mathrm{P}_{\mathrm{s}}$; so $\Omega_{\mathrm{k}^{*}}$ is inconsistent. This is impossible; reject the assumption: $\Omega_{k}$ is consistent.

(iv) Suppose $\Omega_{\mathrm{k}}$ is $\Omega_{\mathrm{k}^{*}} \cup\left\{\mathrm{P}_{\mathrm{s} / \mathrm{t}}, \neg \mathrm{Q}_{\mathrm{t}}\right\}$. In this case, as above, $\Omega_{\mathrm{k} *}$ is consistent and by construction, $\neg(P>Q)_{s} \in \Omega_{k^{*}}$. Suppose $\Omega_{k}$ is inconsistent. Then there are $A_{\mathfrak{u}}$ and $\neg A_{\mathfrak{u}}$ such that $\Omega_{k^{*}} \cup$ $\left\{\mathrm{P}_{\mathrm{s} / \mathrm{t}}, \neg \mathrm{Q}_{\mathrm{t}}\right\} \vdash_{\mathrm{NCx}}^{*} A_{\mathrm{u}}$ and $\Omega_{\mathrm{k}^{*}} \cup\left\{\mathrm{P}_{\mathrm{s} / \mathrm{t}}, \neg \mathrm{Q}_{\mathrm{t}}\right\} \vdash_{\mathrm{NCx}}^{*} \neg A_{\mathrm{u}}$. So reason as follows,

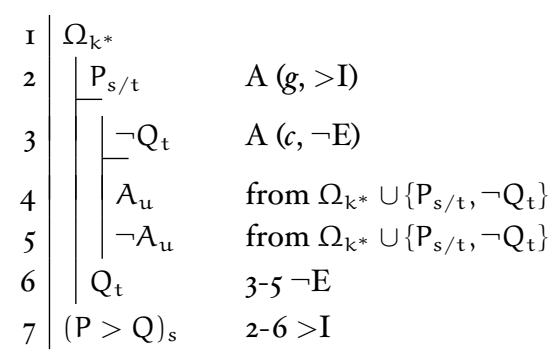


where, by construction, $\mathrm{t}$ is not in $\Omega_{\mathrm{k}^{*}}$. So $\Omega_{\mathrm{k}^{*}} \vdash_{\mathrm{NCx}}^{*}(\mathrm{P}>\mathrm{Q})_{s}$; but $\neg(\mathrm{P}>\mathrm{Q})_{\mathrm{s}} \in \Omega_{\mathrm{k}^{*}}$; so $\Omega_{\mathrm{k}^{*}} \vdash_{\mathrm{NCx}}^{*} \neg(\mathrm{P}>\mathrm{Q})_{s}$; so $\Omega_{\mathrm{k}^{*}}$ is inconsistent. This is impossible; reject the assumption: $\Omega_{k}$ is consistent.

For any $i, \Omega_{i}$ is consistent.

L4.6 If $\Gamma_{0}$ is consistent, then $\Gamma^{\prime}$ is consistent.

Reasoning parallel to $\mathrm{L}_{2} .6$ for $N K \alpha$.

L4.7 If $\Gamma_{0}$ is consistent, then $\Gamma^{\prime}$ is a scapegoat set for $\square$ and $>$.

For $\square$. Suppose $\Gamma_{0}$ is consistent and $\Gamma^{\prime} \vdash_{\mathrm{NCx}}^{*} \neg \square \mathrm{P}_{s}$. By L4.6, $\Gamma^{\prime}$ is consistent; and by the constraints on subscripts, $s$ is included in $\Gamma^{\prime}$. Since $\Gamma^{\prime}$ is consistent, $\Gamma^{\prime} \nvdash_{\mathrm{NCx}}^{*} \neg \neg \square \mathrm{P}_{s}$; so there is a stage in the construction process where $\Omega_{i^{*}}=\Omega_{i-1} \cup\left\{\neg \square P_{s}\right\}$ and $\Omega_{i}=\Omega_{i^{*}} \cup\left\{\neg P_{t}\right\}$; so by construction, $\neg \mathrm{P}_{\mathrm{t}} \in \Gamma^{\prime}$; so $\Gamma^{\prime} \vdash_{\mathrm{NCx}}^{*} \neg \mathrm{P}_{\mathrm{t}}$. So $\Gamma^{\prime}$ is a scapegoat set for $\square$.

For $>$. Suppose $\Gamma_{0}$ is consistent and $\Gamma^{\prime} \vdash_{\mathrm{NCx}}^{*} \neg(\mathrm{P}>\mathrm{Q})_{\mathrm{s}}$. By L4.6, $\Gamma^{\prime}$ is consistent; and by the constraints on subscripts, $s$ is included in $\Gamma^{\prime}$. Since $\Gamma^{\prime}$ is consistent, $\Gamma^{\prime} \nvdash_{\mathrm{NCx}}^{*} \neg \neg(\mathrm{P}>\mathrm{Q})_{s}$; so there is a stage in the construction process where $\Omega_{i^{*}}=\Omega_{i-1} \cup\left\{\neg(P>Q)_{s}\right\}$ and $\Omega_{i}=\Omega_{i^{*}} \cup$ $\left\{\mathrm{P}_{\mathrm{s} / \mathrm{t}}, \neg \mathrm{Q}_{\mathrm{t}}\right\}$; so by construction, $\mathrm{P}_{\mathrm{s} / \mathrm{t}} \in \Gamma^{\prime}$ and $\neg \mathrm{Q}_{\mathrm{t}} \in \Gamma^{\prime}$; so $\Gamma^{\prime} \vdash_{\mathrm{NCx}}^{*} \mathrm{P}_{\mathrm{s} / \mathrm{t}}$ and $\Gamma^{\prime} \vdash_{\mathrm{NCx}}^{*} \neg \mathrm{Q}_{\mathrm{t}}$. So $\Gamma^{\prime}$ is a scapegoat set for $>$.

$\mathrm{C}(\mathrm{I})$ We construct an interpretation $\mathrm{I}=\left\langle\mathrm{W},\left\{\mathrm{R}_{\mathrm{A}} \mid \mathrm{A} \in \mathfrak{I}\right\}, v\right\rangle$ based on $\Gamma^{\prime}$ as follows. Let $W$ have a member $w_{s}$ corresponding to each subscript $s$ included in $\Gamma^{\prime}$, except that in $C_{I}$, if there is some $A$ such that $\Gamma^{\prime} \vdash_{\mathrm{NC} 1}^{*} A_{S}$ and $\Gamma^{\prime} \vdash_{\mathrm{NC} 1}^{*} A_{\mathrm{s} / \mathrm{t}}$ then $w_{\mathrm{s}}=w_{\mathrm{t}}$, and in $C_{2}$, if there is some $A$ such that $\Gamma^{\prime} \vdash_{\mathrm{NC2}}^{*} A_{\mathrm{s} / \mathrm{t}}$ and $\Gamma^{\prime} \vdash_{\mathrm{NC2}}^{*} A_{\mathrm{s} / \mathrm{u}}$ then $w_{\mathrm{t}}=w_{\mathrm{u}}$ (we could do this, in the usual way, by establishing equivalence classes from members of $W$ ). Then $\left\langle w_{s}, w_{t}\right\rangle \in R_{A}$ iff $\Gamma^{\prime} \vdash_{\mathrm{NCx}}^{*} A_{s / t}$; and $v_{w_{s}}(p)=1$ iff $\Gamma^{\prime} \vdash_{\mathrm{NCx}}^{*} p_{s}$.

Note that the specification is consistent for $C_{I}$ and $C_{2}$ : Say $\mathcal{P}_{(s)}$ is some $p_{s}, P_{s / v}, P_{v / s}$ or $P_{s / s}$. (i) Suppose $w_{s}=w_{t}$ and $\Gamma^{\prime} \vdash_{\mathrm{NC} 1}^{*} \mathcal{P}_{(s)}$. Since $w_{s}=$ $w_{\mathrm{t}}$ there is some $A$ such that $\Gamma^{\prime} \vdash_{\mathrm{NC} 1}^{*} A_{s}$ and $\Gamma^{\prime} \vdash_{\mathrm{NC} 1}^{*} A_{s / t}$; so by AMDL, $\Gamma^{\prime} \vdash_{\mathrm{NC} 1}^{*} \mathcal{P}_{(\mathrm{t})}$. And similarly if $w_{\mathrm{s}}=w_{\mathrm{t}}$ and $\Gamma^{\prime} \vdash_{\mathrm{NC} 1}^{*} \mathcal{P}_{(\mathrm{t})}$, then $\Gamma^{\prime} \vdash_{\mathrm{NC} 1}^{*} \mathcal{P}_{(\mathrm{s})}$. (ii) Suppose $w_{\mathrm{t}}=w_{\mathrm{u}}$ and $\Gamma^{\prime} \vdash_{\mathrm{NC2}}^{*} \mathcal{P}_{(\mathrm{t})}$. Since $w_{\mathrm{t}}=w_{\mathrm{u}}$, there is some $A$ such that $\Gamma^{\prime} \vdash_{\mathrm{NC} 2}^{*} A_{\mathrm{s} / \mathrm{t}}$ and $\Gamma^{\prime} \vdash_{\mathrm{NC} 2}^{*} A_{\mathrm{s} / \mathrm{u}}$; so by AMRs, $\Gamma^{\prime} \vdash_{\mathrm{NC} 2}^{*} \mathcal{P}_{(\mathrm{u})}$. And similarly if $w_{\mathfrak{t}}=w_{\mathfrak{u}}$ and $\Gamma^{\prime} \vdash_{\mathrm{NC} 2}^{*} \mathcal{P}_{(\mathfrak{u})}$, then $\Gamma^{\prime} \vdash_{\mathrm{NC} 2}^{*} \mathcal{P}_{(\mathfrak{t})}$.

$\mathrm{L}_{4} .8$ If $\Gamma_{0}$ is consistent then for $\left\langle\mathrm{W},\left\{\mathrm{R}_{\mathrm{A}} \mid A \in \mathfrak{I}\right\}, v\right\rangle$ constructed as above, and for any $s$ included in $\Gamma^{\prime}, v_{w_{s}}(A)=1$ iff $\Gamma^{\prime} \vdash_{\mathrm{NCx}}^{*} A_{s}$.

Suppose $\Gamma_{0}$ is consistent and $s$ is included in $\Gamma^{\prime}$. By $\mathrm{L}_{4} \cdot 4, \Gamma^{\prime}$ is s-maximal. By L4. 6 and $\mathrm{L}_{4.7}, \Gamma^{\prime}$ is consistent and a scapegoat set for $\square$ and $>$. Now by induction on the number of operators in $A_{s}$, 
Basis: If $A_{s}$ has no operators, then it is a parameter $p_{s}$ and by construction, $v_{w_{s}}(p)=1$ iff $\Gamma^{\prime} \vdash_{\mathrm{NCx}}^{*} p_{s}$. So $v_{w_{s}}(A)=1$ iff $\Gamma^{\prime} \vdash_{\mathrm{NCx}}^{*} A_{s}$.

Assp: For any $i, 0 \leqslant i<k$, if $A_{s}$ has $i$ operators, then $v_{w_{s}}(A)=1$ iff $\Gamma^{\prime} \vdash_{\mathrm{NCx}}^{*} A_{s}$.

Show: If $A_{s}$ has $k$ operators, then $v_{w_{s}}(A)=1$ iff $\Gamma^{\prime} \vdash_{\mathrm{NCx}}^{*} A_{s}$.

If $A_{s}$ has $k$ operators, then it is of the form $\neg P_{s},(P \supset Q)_{s}$, $(\mathrm{P} \wedge \mathrm{Q})_{s},(\mathrm{P} \vee \mathrm{Q})_{s},(\mathrm{P} \equiv \mathrm{Q})_{s}, \square \mathrm{P}_{s}, \diamond \mathrm{P}_{\mathrm{s}}$ or $(\mathrm{P}>\mathrm{Q})_{s}$ where $\mathrm{P}$ and $\mathrm{Q}$ have $<k$ operators.

$(\neg) A_{s}$ is $\neg P_{s}$. (i) Suppose $v_{w_{s}}(A)=1$; then $v_{w_{s}}(\neg P)=1$; so by $\mathrm{TC}(\neg), v_{w_{s}}(\mathrm{P})=0$; so by assumption, $\Gamma^{\prime}{H_{\mathrm{NCx}}^{*}}^{*} \mathrm{P}_{s}$; so by $s-$ maximality, $\Gamma^{\prime} \vdash_{\mathrm{NCx}}^{*} \neg \mathrm{P}_{s}$, where this is to say, $\Gamma^{\prime} \vdash_{\mathrm{NCx}}^{*} A_{s}$. (ii) Suppose $\Gamma^{\prime} \vdash_{\mathrm{NCx}}^{*} A_{s}$; then $\Gamma^{\prime} \vdash_{\mathrm{NCx}}^{*} \neg \mathrm{P}_{s}$; so by consistency, $\Gamma^{\prime} \vdash_{\mathrm{NCx}}^{*} \mathrm{P}_{s}$; so by assumption, $v_{w_{s}}(\mathrm{P})=0$; so by $\mathrm{TC}(\neg), v_{w_{s}}(\neg \mathrm{P})=1$, where this is to say, $v_{w_{s}}(A)=1$. So $v_{w_{s}}(A)=1 \mathrm{iff} \Gamma^{\prime} \vdash_{\mathrm{NCx}}^{*} A_{s}$.

(

( $\square) A_{s}$ is $\square P_{s}$. (i) Suppose $v_{w_{s}}(A)=1$ but $\Gamma^{\prime} \nvdash_{N C x}^{*} A_{s}$; then $v_{w_{s}}(\square P)=$ 1 but $\Gamma^{\prime}{H_{N C x}^{*}}^{*} \square P_{s}$. From the latter, by s-maximality, $\Gamma^{\prime} \vdash_{\mathrm{NCx}}^{*} \neg \square \mathrm{P}_{s}$; so, since $\Gamma^{\prime}$ is a scapegoat set for $\square$, there is some $t$ such that $\Gamma^{\prime} \vdash_{\mathrm{NCx}}^{*} \neg \mathrm{P}_{\mathrm{t}}$; so by consistency, $\Gamma^{\prime} \nvdash_{\mathrm{NCx}}^{*} \mathrm{P}_{\mathrm{t}}$; so by assumption, $v_{w_{\mathrm{t}}}(\mathrm{P})=0$; so by $\mathrm{TC}(\square)_{v}, v_{w_{s}}(\square \mathrm{P})=0$. This is impossible; reject the assumption: if $v_{w_{s}}(A)=1$, then $\Gamma^{\prime} \vdash_{\mathrm{NCx}}^{*} A_{s}$.

(ii) Suppose $\Gamma^{\prime} \vdash_{\mathrm{NCx}}^{*} A_{s}$ but $v_{w_{s}}(A)=0$; then $\Gamma^{\prime} \vdash_{\mathrm{NCx}}^{*} \square \mathrm{P}_{\mathrm{s}}$ but $v_{w_{s}}(\square \mathrm{P})=0$. From the the latter, by $\mathrm{TC}(\square)_{v}$, there is some $w_{\mathrm{t}} \in \mathrm{W}$ such that $v_{w_{\mathrm{t}}}(\mathrm{P})=0$; so by assumption, $\Gamma^{\prime} \nvdash_{\mathrm{NCx}}^{*} \mathrm{P}_{\mathrm{t}}$; but since $w_{t} \in W$, by construction, $t$ appears in $\Gamma^{\prime}$ so by ( $\square \mathrm{Ev}$ ), $\Gamma^{\prime} \vdash_{\mathrm{NCx}}^{*} P_{\mathrm{t}}$. This is impossible; reject the assumption: if $\Gamma^{\prime} \vdash_{\mathrm{NCx}} A_{s}$ then $v_{w_{s}}(A)=1$. So $v_{w_{s}}(A)=1$ iff $\Gamma^{\prime} \vdash_{\mathrm{NCx}}^{*} A_{s}$.

$(\diamond)$

$(>) A_{s}$ is $(P>Q)_{s}$. Suppose $v_{w_{s}}(A)=1$ but $\Gamma^{\prime} \nvdash_{N C x}^{*} A_{s}$; then $v_{w_{s}}(\mathrm{P}>\mathrm{Q})=1$ but $\Gamma^{\prime} \forall_{\mathrm{NCx}}^{*}(\mathrm{P}>\mathrm{Q})_{s}$. From the latter, by $s-$ maximality, $\Gamma^{\prime} \vdash_{\mathrm{NCx}}^{*} \neg(\mathrm{P}>\mathrm{Q})_{s}$; so, since $\Gamma^{\prime}$ is a scapegoat set for $>$, there is some $t$ such that $\Gamma^{\prime} \vdash_{\mathrm{NCx}}^{*} \mathrm{P}_{\mathrm{s} / \mathrm{t}}$ and $\Gamma^{\prime} \vdash_{\mathrm{NCx}}^{*} \neg \mathrm{Q}_{\mathrm{t}}$; from the first, by construction, $\left\langle w_{s}, w_{t}\right\rangle \in R_{P}$; and from the second, by consistency, $\Gamma^{\prime} \nvdash_{N C x}^{*} Q_{t}$; so by assumption, $v_{w_{t}}(Q)=0$; so by $\mathrm{TC}(>), v_{w_{s}}(\mathrm{P}>\mathrm{Q})=0$. This is impossible; reject the assumption: if $v_{w_{s}}(A)=1$, then $\Gamma^{\prime} \vdash_{\mathrm{NCx}}^{*} A_{s}$.

(ii) Suppose $\Gamma^{\prime} \vdash_{\mathrm{NCx}}^{*} A_{s}$ but $v_{w_{s}}(A)=0$; then $\Gamma^{\prime} \vdash_{\mathrm{NCx}}^{*}(\mathrm{P}>\mathrm{Q})_{\mathrm{s}}$ but $v_{w_{s}}(\mathrm{P}>\mathrm{Q})=0$. From the the latter, by $\mathrm{TC}(>)$, there is 
some $w_{\mathrm{t}} \in \mathrm{W}$ such that $\left\langle w_{\mathrm{s}}, w_{\mathrm{t}}\right\rangle \in \mathrm{R}_{\mathrm{P}}$ and $v_{w_{\mathrm{t}}}(\mathrm{Q})=0$; from the first of these, by construction, $\Gamma^{\prime} \vdash_{\mathrm{NCx}}^{*} \mathrm{P}_{\mathrm{s} / \mathrm{t}}$; and from the second, by assumption, $\Gamma^{\prime} \nvdash_{\mathrm{NCx}}^{*} \mathrm{Q}_{\mathrm{t}}$; but by $(>\mathrm{E}), \Gamma^{\prime} \vdash_{\mathrm{NCx}}^{*} \mathrm{Q}_{\mathrm{t}}$. This is impossible; reject the assumption: if $\Gamma^{\prime} \vdash_{\mathrm{NCx}} A_{s}$ then $v_{w_{s}}(A)=$ 1. So $v_{w_{\mathrm{s}}}(A)=1$ iff $\Gamma^{\prime} \vdash_{\mathrm{NCx}}^{*} A_{s}$.

For any $A_{s}, v_{w_{s}}(A)=1$ iff $\Gamma^{\prime} \vdash_{\mathrm{NCx}}^{*} A_{s}$.

L4.9 If $\Gamma_{0}$ is consistent, then $\left\langle W,\left\{R_{A} \mid A \in \mathfrak{I}\right\}, v\right\rangle$ constructed as above is a $C x$ interpretation.

In each case, we need to show that the interpretation meets the condition(s) $x$. Suppose $\Gamma_{0}$ is consistent.

(I) If (I) is in $C x$, then AMPr is in NCx. Suppose $w_{t} \in f_{A}\left(w_{s}\right)$; then $\left\langle w_{s}, w_{t}\right\rangle \in R_{A}$; so by construction, $\Gamma^{\prime} \vdash_{\mathrm{NCx}}^{*} A_{s / t}$; so by AMPI, $\Gamma^{\prime} \vdash_{\mathrm{NCx}}^{*} A_{\mathrm{t}}$; so by $\mathrm{L}_{4} .8, v_{w_{\mathrm{t}}}(A)=1$; $\operatorname{so}_{\mathrm{t}} \in[A]$. So $\mathrm{f}_{A}\left(w_{\mathrm{s}}\right) \subseteq[A]$.

(2) If (2) is in $C x$ then $A_{M P_{2}}$ is in $N C x$. Suppose $w_{s} \in[A]$; then $v_{w_{s}}(A)=1$; so by $\mathrm{L}_{4} .8, \Gamma^{\prime} \vdash_{\mathrm{NCx}}^{*} A_{s}$; so by $\mathrm{AMP}_{2}, \Gamma^{\prime} \vdash_{\mathrm{NCx}}^{*} A_{s / s}$; so by construction, $\left\langle w_{s}, w_{s}\right\rangle \in R_{A}$; so $w_{s} \in f_{A}\left(w_{s}\right)$.

(3) If (3) is in $C x$ then AMs is in $N C x$. Suppose $[A] \neq \phi$ but $f_{A}\left(w_{s}\right)=$ $\phi$. From the former, there is some $w_{t} \in W$ such that $v_{w_{t}}(A)=1$; so by $\mathrm{L}_{4} .8, \Gamma^{\prime} \vdash_{\mathrm{NCx}}^{*} A_{\mathrm{t}}$; so by $(\diamond \mathrm{I} v), \Gamma^{\prime} \vdash_{\mathrm{NCx}}^{*} \diamond A_{s}$. From the latter, there is no $w_{u}$ such that $w_{s} R_{A} w_{u}$; so there is no $w_{u}$ such that $w_{s} R_{A} w_{u}$ and $v_{w_{u}}(B)=0$, and there is no $w_{u}$ such that $w_{s} R_{A} w_{u}$ and $v_{w_{u}}(\neg B)=0$; so by $\mathrm{TC}(>), v_{w_{s}}(A>B)=1$ and $v_{w_{s}}(A>\neg B)=1$; so by $L_{4} .8, \Gamma^{\prime} \vdash_{\mathrm{NCx}}^{*}(\mathrm{~A}>\mathrm{B})_{\mathrm{s}}$ and $\Gamma^{\prime} \vdash_{\mathrm{NCx}}^{*}(A>\neg \mathrm{B})_{s}$. So reason as follows,

\begin{tabular}{|c|c|c|}
\hline I & $\Gamma^{\prime}$ & \\
\hline 2 & $\diamond A_{s}$ & from $\Gamma^{\prime}$ \\
\hline 3 & $(A>B)_{s}$ & from $\Gamma^{\prime}$ \\
\hline 4 & $(A>\neg B)_{s}$ & from $\Gamma^{\prime}$ \\
\hline & $A_{s / t}$ & $\mathrm{~A}\left(\mathrm{~g}, 2 \mathrm{AM \textrm {S } _ { \mathrm { I } } )}\right.$ \\
\hline 6 & $\diamond A_{s}$ & $\mathrm{~A}(c, \neg \mathrm{I})$ \\
\hline & $B_{t}$ & $3,5>E$ \\
\hline & $\neg \mathrm{B}_{\mathrm{t}}$ & $4,5>E$ \\
\hline & $\neg \diamond A_{s}$ & $6-8 \neg \mathrm{I}$ \\
\hline & $\neg \diamond A_{s}$ & $2,5-9 \mathrm{AMS}_{\mathrm{I}}$ \\
\hline
\end{tabular}

So $\Gamma^{\prime} \vdash_{\mathrm{NCx}}^{*} \neg \diamond A_{s}$; and since by $\mathrm{L}_{4} \cdot 6, \Gamma^{\prime}$ is consistent, $\Gamma^{\prime} \nvdash_{\mathrm{NCx}}^{*}$ $\diamond A_{s}$. This is impossible; reject the assumption: if $[A] \neq \phi$, then $\mathrm{f}_{\mathrm{A}}\left(w_{\mathrm{s}}\right) \neq \phi$.

(4) If (4) is in $C x$ then $A M s_{2}$ is in $N C x$. Suppose $f_{A}\left(w_{s}\right) \subseteq[B]$ and $f_{B}\left(w_{s}\right) \subseteq[A]$. Then any $x \in W$ such that $w_{s} R_{A} x$ has 
$v_{x}(B)=1$ and any $y \in W$ such that $w_{s} R_{B} y$ has $v_{y}(A)=1$; so by $\mathrm{TC}(>), v_{w_{s}}(\mathrm{~A}>\mathrm{B})=1$ and $v_{w_{s}}(\mathrm{~B}>\mathrm{A})=1$; so by $\mathrm{L}_{4} .8$, $\Gamma^{\prime} \vdash_{\mathrm{NCx}}^{*}(A>B)_{s}$ and $\Gamma^{\prime} \vdash_{\mathrm{NCx}}^{*}(\mathrm{~B}>A)_{s}$. Suppose $w_{\mathrm{t}} \in \mathrm{f}_{\mathrm{A}}\left(w_{s}\right)$; then by construction, $\Gamma^{\prime} \vdash_{\mathrm{NCx}}^{*} A_{s / t}$; so by AMs $2, \Gamma^{\prime} \vdash_{\mathrm{NCx}}^{*} \mathrm{~B}_{s / \mathrm{t}}$; so by construction, $w_{t} \in f_{B}\left(w_{s}\right)$. Suppose $w_{t} \in f_{B}\left(w_{s}\right)$; then by construction, $\Gamma^{\prime} \vdash_{\mathrm{NCx}}^{*} B_{s / t}$; so by $\mathrm{AMs}_{2}, \Gamma^{\prime} \vdash_{\mathrm{NCx}}^{*} A_{s / \mathrm{t}}$; so by construction, $w_{t} \in f_{A}\left(w_{s}\right)$. So $f_{A}\left(w_{s}\right)=f_{B}\left(w_{s}\right)$.

(5) If (5) is in $C x$ then $A M s_{3}$ is in $N C x$. Suppose $f_{A}\left(w_{s}\right) \cap[B] \neq \phi$ but $f_{A \wedge B}\left(w_{s}\right) \nsubseteq f_{A}\left(w_{s}\right)$. From the former, there is some $w_{t} \in$ $\mathrm{f}_{\mathrm{A}}\left(w_{\mathrm{S}}\right)$ such that $v_{w_{\mathrm{t}}}(\mathrm{B})=1$; so by $\mathrm{TC}(\neg), v_{w_{\mathrm{t}}}(\neg \mathrm{B})=0$; so by $\mathrm{TC}(>), v_{w_{s}}(\mathrm{~A}>\neg \mathrm{B})=0$; so by $\mathrm{TC}(\neg), v_{w_{s}}(\neg(\mathrm{A}>\neg \mathrm{B}))=$ 1; so by $\mathrm{L}_{4} .8, \Gamma^{\prime} \vdash_{\mathrm{NCx}}^{*} \neg(A>\neg \mathrm{B})_{s}$. From the latter, there is some $w_{\mathfrak{u}}$ such that $w_{\mathfrak{u}} \in \mathrm{f}_{\mathrm{A} \wedge \mathrm{B}}\left(w_{s}\right)$ but $w_{\mathfrak{u}} \notin \mathrm{f}_{A}\left(w_{s}\right)$. From the first of these, by construction, $\Gamma^{\prime} \vdash_{\mathrm{NCx}}^{*}(A \wedge B)_{s / u}$; so by $\mathrm{AMs}_{3}, \Gamma^{\prime} \vdash_{\mathrm{NCx}}^{*} A_{\mathrm{s} / \mathrm{u}}$; so by construction, $w_{\mathrm{u}} \in \mathrm{f}_{\mathrm{A}}\left(w_{\mathrm{s}}\right)$. This is impossible; reject the assumption: if $f_{A}\left(w_{S}\right) \cap[B] \neq \phi$ then $f_{A \wedge B}\left(w_{s}\right) \subseteq f_{A}\left(w_{s}\right)$.

(6) Suppose (6) is in $C x, w_{t} \in f_{A}\left(w_{s}\right)$ and $w_{u} \in f_{A}\left(w_{s}\right)$. Then by construction, $\Gamma^{\prime} \vdash_{\mathrm{NCx}}^{*} A_{s / t}$ and $\Gamma^{\prime} \vdash_{\mathrm{NCx}}^{*} A_{s / u}$; and by construction, since we are in $C_{2}, w_{\mathrm{t}}=w_{\mathrm{u}}$.

(7) Suppose (7) is in $C x, w_{s} \in[A]$ and $w_{t} \in f_{A}\left(w_{s}\right)$. Since $w_{s} \in[A]$, $v_{w_{s}}(A)=1$; so by $L_{4} .8, \Gamma^{\prime} \vdash_{\mathrm{NCx}}^{*} A_{s}$; and since $w_{\mathrm{t}} \in \mathrm{f}_{\mathrm{A}}\left(w_{s}\right)$, by construction, $\Gamma^{\prime} \vdash_{\mathrm{NCx}}^{*} A_{\mathrm{s} / \mathrm{t}}$. So by construction, since we are in $C_{I}, w_{\mathrm{s}}=w_{\mathrm{t}}$.

MAP For any $w_{s} \in W$, set $m(s)=w_{s}$; otherwise $m(s)$ is arbitrary.

L4.I I If $\Gamma_{0}$ is consistent, then $v_{m}\left(\Gamma_{0}\right)=1$.

Reasoning parallel to $\mathrm{L} 2$. Io for $N K \alpha$.

Main result: Suppose $\Gamma \models_{C_{x}} A$ but $\Gamma \nvdash_{N C x} A$. Then $\Gamma_{0} \models_{C x}^{*} A_{0}$ but $\Gamma_{0} \nvdash_{N C x}^{*} A_{0}$. By $(\mathrm{DN})$, if $\Gamma_{0} \vdash_{\mathrm{NCx}}^{*} \neg \neg A_{0}$, then $\Gamma_{0} \vdash_{\mathrm{NCx}}^{*} A_{0}$; so $\Gamma_{0} \nvdash_{\mathrm{NCx}}^{*} \neg \neg A_{0}$; so by $\mathrm{L}_{4.2}$, $\Gamma_{0} \cup\left\{\neg A_{0}\right\}$ is consistent; so by $\mathrm{L}_{4.9}$ and $\mathrm{L}_{4} . \mathrm{IO}$, there is a $C x$ interpretation $\left\langle\mathrm{W},\left\{\mathrm{R}_{\mathrm{A}} \mid \mathrm{A} \in \mathfrak{I}\right\}, v\right\rangle_{\mathrm{m}}$ constructed as above such that $v_{\mathrm{m}}\left(\Gamma_{0} \cup\left\{\neg \mathrm{A}_{0}\right\}\right)=1$; so $v_{m(0)}(\neg A)=1$; so by TC $(\neg), v_{m(0)}(A)=0$; so $v_{m}\left(\Gamma_{0}\right)=1$ and $v_{m(0)}(A)=0$; so by $\mathrm{VCx}^{*}, \Gamma_{0} \forall_{C x}^{*} A_{0}$. This is impossible; reject the assumption: if $\Gamma \models_{C x} A$, then $\Gamma \vdash_{\mathrm{NCx}}$ A.

5 intuitionistic logic: $I L$ (CH. 6)

\section{I LANGUAGE / SEMANTIC NOTIONS}

LIL The vOCABULARY consists of propositional parameters $p_{0}, p_{1} \ldots$ with the operators, $\wedge, \vee, \neg$, and $\sqsupset$. Each propositional parameter is a FORMULA; if $A$ and $B$ are formulas, so are $(A \wedge B),(A \vee B), \neg A$, and $(A \sqsupset B)$. 
IIL An InterpRETATION is a triple $\langle W, R, v\rangle$ where $W$ is a set of worlds, $R$ is a subset of $W^{2}=W \times W$, and $v$ is a function such that for any $w \in W$ and $p, v_{w}(p)=1$ or $v_{w}(p)=0$. For $x, y, z \in W$, an interpretation is subject to the conditions,

$$
\begin{array}{lll}
\rho & \text { for all } x, x R x & \text { reflexivity } \\
\tau & \text { for all } x, y, z, \text { if } x \text { Ry and } y R z \text { then } x R z & \text { transitivity } \\
h & \text { for any parameter } p, \text { if } \nu_{x}(p)=1, \text { and } x R y, & \text { heredity } \\
& \text { then } v_{y}(p)=1 &
\end{array}
$$

We think of worlds as representing a state of information at a given time. $v_{w}(p)=1$ when $p$ is proved at state $w$. The heredity condition guarantees that what is proved at one stage remains proved at the next. Notice that $v_{w}(p)=0$ does not indicate that $p$ is false - but rather that p isn'tproved.

TIL For complex expressions,

$(\wedge) v_{w}(A \wedge B)=1$ if $v_{w}(A)=1$ and $v_{w}(B)=1$, and 0 otherwise.

$(\vee) v_{w}(A \vee B)=1$ if $v_{w}(A)=1$ or $v_{w}(B)=1$, and 0 otherwise.

$\left(\rightarrow v_{w}(\neg A)=1\right.$ if all $x \in W$ such that $w$ Rx have $v_{x}(A)=0$, and 0 otherwise.

( $\sqsupset) v_{w}(A \sqsupset B)=1$ if all $x \in W$ such that $w$ Rx have either $v_{x}(A)=0$ or $v_{x}(B)=1$, and 0 otherwise.

For a set $\Gamma$ of formulas, $v_{w}(\Gamma)=1$ iff $v_{w}(A)=1$ for each $A \in \Gamma$; then,

VIL $\Gamma \models_{\mathrm{IL}} A$ iff there is no $I L$ interpretation $\langle\mathrm{W}, \mathrm{R}, v\rangle$ and $w \in W$ such that $v_{w}(\Gamma)=1$ and $v_{w}(\mathrm{~A})=0$.

\subsection{NATURAL Derivations: NIL}

Augment the language for intuionistic logic to include expressions with subscripts and expressions of the sort s.t as for $N K \alpha$, along with a unary operator, $\sim$. Intuitively, $\sim A$ indicates that $A$ is not (yet) proven. There is one new rule for the heredity condition. Otherwise, rules are as in $N K \rho \tau$ with $\sim$ like $\neg$, and rules for $\sqsupset$ and $\neg$ on the analogy of -3 and $\square \neg$.

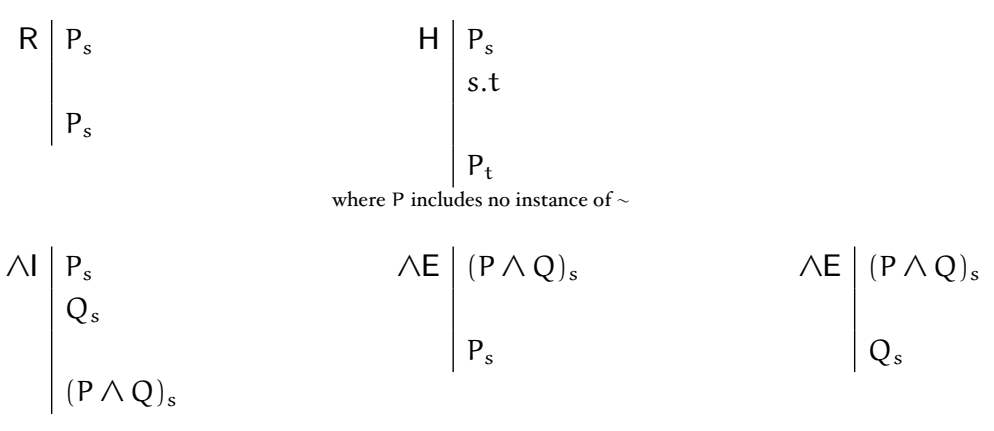


$\vee \mathbf{I} \mid \begin{aligned} & P_{s} \\ & (P \vee Q)_{s}\end{aligned}$

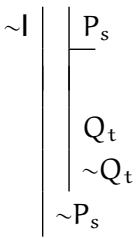

$\sqsupset \mathbf{l}||$ s.t

$P_{t}$

$Q_{\mathrm{t}}$

$(\mathrm{P} \sqsupset \mathrm{Q})_{s}$

where $t$ does not appear in any undischarged premise or assumption

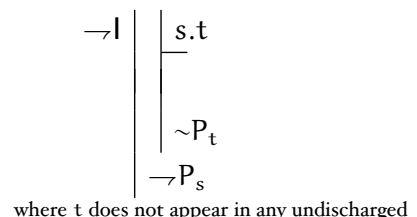

where $t$ does not appear in any undischarged premise or assumption
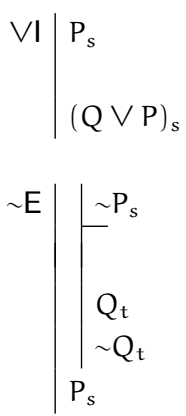

$\sqsupset \mathrm{E} \mid \begin{aligned} & (\mathrm{P} \sqsupset \mathrm{Q})_{\mathrm{s}} \\ & \mathrm{s} . \mathrm{t} \\ & \mathrm{P}_{\mathrm{t}} \\ & \mathrm{Q}_{\mathrm{t}}\end{aligned}$

$\neg \mathrm{E} \mid \begin{aligned} & \neg \mathrm{P}_{\mathrm{s}} \\ & \text { s.t } \\ & \sim \mathrm{P}_{\mathrm{t}}\end{aligned}$

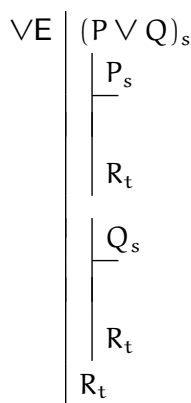

$\mathrm{AM} \rho$ s.s

$\mathrm{AM \tau} \mid$ s.t

t.u s.u

Every subscript is 0, appears in a premise, or appears in the t-place of an accessible assumption for $\sqsupset \mathrm{I}$ or $\neg \mathrm{I}$. Where the members of $\Gamma$ and $A$ are formulas in the original language for intuitionistic logic (without subscripts and without $\sim$ ), let let the members of $\Gamma_{0}$ be the formulas in $\Gamma$, each with subscript 0 . Then,

NIL $\Gamma \vdash_{\mathrm{NIL}} A$ iff there is an NIL derivation of $A_{0}$ from the members of $\Gamma_{0}$.

As examples, here are instances of the more interesting standard axioms for intuitionistic logic. Note that our account of a derivation guarantees that $\sim$ is not an operator in any of $A, B$, or $C$.

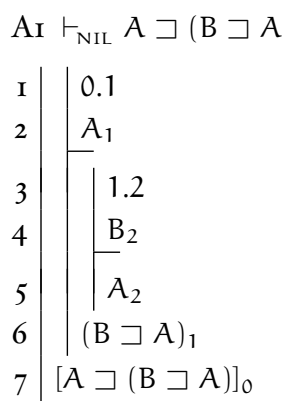
$\mathrm{A}(g, \sqsupset \mathrm{I})$
A $(g, \sqsupset \mathrm{I})$
2,3 $\mathrm{H}$
3-5 $\sqsupset \mathrm{I}$
$\mathrm{I}-6 \sqsupset \mathrm{I}$ 
$\mathrm{A} 2 \vdash_{\mathrm{NIL}}(\mathrm{A} \sqsupset \mathrm{B}) \sqsupset[(\mathrm{A} \sqsupset(\mathrm{B} \sqsupset \mathrm{C})) \sqsupset(\mathrm{A} \sqsupset \mathrm{C})]$

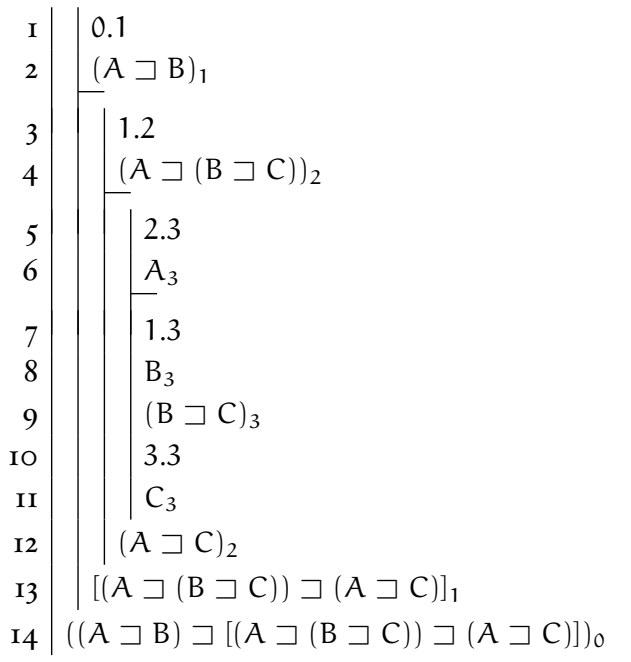

$\mathrm{A}(g, \sqsupset \mathrm{I})$

$\mathrm{A}(g, \sqsupset \mathrm{I})$

$\mathrm{A}(g, \sqsupset \mathrm{I})$

3,5 $\mathrm{AM \tau}$

$2,7,6 \sqsupset \mathrm{E}$

$4,5,6 \sqsupset \mathrm{E}$

$\mathrm{AM} \rho$

9,IO,8 $\sqsupset \mathrm{E}$

5-II $\sqsupset \mathrm{I}$

3-I2 $\sqsupset \mathrm{I}$

I-I3 $\sqsupset \mathrm{I}$

$\mathrm{A}_{3} \vdash_{\mathrm{NIL}} A \sqsupset(\mathrm{B} \sqsupset(\mathrm{A} \wedge \mathrm{B}))$

$\mathrm{A}_{4} \vdash_{\mathrm{NIL}}(\mathrm{A} \wedge \mathrm{B}) \sqsupset \mathrm{A}$

$\mathrm{A} 5 \vdash_{\mathrm{NIL}}(\mathrm{A} \wedge \mathrm{B}) \sqsupset \mathrm{B}$

$\mathrm{A} 6 \vdash_{\mathrm{NIL}} A \sqsupset(\mathrm{A} \vee \mathrm{B})$

$\mathrm{A}_{7} \vdash_{\mathrm{NIL}} \mathrm{B} \sqsupset(\mathrm{A} \vee \mathrm{B})$

$\mathrm{A} 8 \vdash_{\mathrm{NIL}}(\mathrm{A} \sqsupset \mathrm{C}) \sqsupset[(\mathrm{B} \sqsupset \mathrm{C}) \sqsupset((\mathrm{A} \vee \mathrm{B}) \sqsupset \mathrm{C})]$

A9 $\vdash_{\mathrm{NIL}}(\mathrm{A} \sqsupset \mathrm{B}) \sqsupset[(\mathrm{A} \sqsupset \rightarrow \mathrm{B}) \sqsupset \rightarrow \mathrm{A}]$

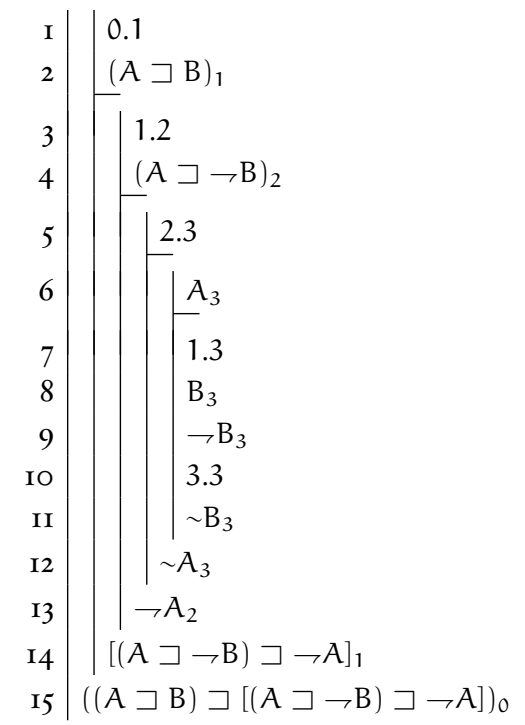

$\mathrm{A}(g, \sqsupset \mathrm{I})$

$\mathrm{A}(g, \sqsupset \mathrm{I})$

A $(g, \neg \mathrm{I})$

$\mathrm{A}(c, \sim \mathrm{I})$

3,5 $\mathrm{AM} \tau$

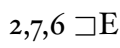

4,5,6 $\sqsupset \mathrm{E}$

$\mathrm{AM} \rho$

9,IO $\rightarrow \mathrm{E}$

6-II $\sim \mathrm{I}$

$5^{-\mathrm{I} 2} \rightarrow \mathrm{I}$

3-I3 $\sqsupset \mathrm{I}$

I-I4 $\mathrm{I}$ 


\begin{tabular}{|c|c|c|}
\hline I & $\mid 0.1$ & $\mathrm{~A}(g, \sqsupset \mathrm{I})$ \\
\hline 2 & $\neg A_{1}$ & \\
\hline 3 & 1.2 & $\mathrm{~A}(g, \sqsupset \mathrm{I})$ \\
\hline 4 & $A_{2}$ & \\
\hline 5 & $\sim \mathrm{B}_{2}$ & $\mathrm{~A}(c, \sim \mathrm{E})$ \\
\hline 6 & $A_{2}$ & ${ }_{4} \mathrm{R}$ \\
\hline 7 & $\sim A_{2}$ & $2,3 \rightarrow \mathrm{E}$ \\
\hline 8 & $\mathrm{~B}_{2}$ & $5-7 \sim \mathrm{E}$ \\
\hline 9 & $(A \sqsupset B)_{1}$ & 3-8 $\mathrm{I}$ \\
\hline IO & $\neg \mathrm{A} \sqsupset(\mathrm{A} \sqsupset \mathrm{B})]_{0}$ & $\mathrm{I}-9 \sqsupset \mathrm{I}$ \\
\hline
\end{tabular}

A system with these axioms and MP (which we already have by $\mathrm{AM} \rho$ with $\sqsupset \mathrm{E}$ ) turns into classical logic if AIo is replaced by double negation, $\neg \neg A \sqsupset A$. But we cannot prove $\neg \neg A \sqsupset A$ (or at least we cannot if our derivation system is sound).

\section{$5 \cdot 3$ SOUNDNESS AND COMPLETENESS}

Preliminaries: Begin with generalized notions of validity to include expressions with subscripts and operator ' $\sim$ '. First, as a supplement to TIL,

TIL $\quad(\sim) v_{w}(\sim A)=1$ if $v_{w}(A)=0$, and 0 otherwise.

For a model $\langle W, R, v\rangle$, let $m$ be a map from subscripts into $W$. Say $\langle W, R, v\rangle_{m}$ is $\langle W, R, v\rangle$ with map $m$. Then, where $\Gamma$ is a set of expressions of our language for derivations, $v_{m}(\Gamma)=1$ iff for each $A_{s} \in \Gamma, v_{m(s)}(A)=1$, and for each s.t $\in$ $\Gamma,\langle\mathrm{m}(\mathrm{s}), \mathrm{m}(\mathrm{t})\rangle \in \mathrm{R}$. Now expand notions of validity to include subscripted formulas, and alternate expressions as indicated in double brackets.

VIL* $\Gamma \models_{\mathbb{L}}^{*} A_{s} \llbracket$ s.t $\rrbracket$ iff there is no $I L$ interpretation $\langle W, R, v\rangle_{m}$ such that $v_{\mathrm{m}}(\Gamma)=1$ but $v_{\mathrm{m}(\mathrm{s})}(\mathrm{A})=0 \llbracket\langle\mathrm{m}(\mathrm{s}), \mathrm{m}(\mathrm{t})\rangle \notin \mathrm{R} \rrbracket$.

NIL* $\Gamma \vdash_{\mathrm{NIL}}^{*} A_{s} \llbracket$ s.t $\rrbracket$ iff there is an NIL derivation of $A_{s} \llbracket$ s.t $\rrbracket$ from the members of $\Gamma$.

These notions reduce to the standard ones when all the members of $\Gamma$ and $A$ have subscript 0 (and so do not include expressions of the sort s.t) and do not include ' '. For the following, cases omitted are like ones worked, and so left to the reader.

THEOREM 5.I NIL is sound: If $\Gamma \vdash_{\mathrm{NIL}}$ A then $\Gamma \models_{\mathrm{IL}}$ A.

L5.I If $\Gamma \subseteq \Gamma^{\prime}$ and $\Gamma \models_{\mathrm{L}}^{*} \mathrm{P}_{\mathrm{s}} \llbracket$ s.t $\rrbracket$, then $\Gamma^{\prime} \models_{\mathrm{L}}^{*} \mathrm{P}_{\mathrm{s}} \llbracket$ s.t $\rrbracket$.

Reasoning parallel to that for L2.I of $N K \alpha$. 
Main result: For each line in a derivation let $\mathcal{P}_{i}$ be the expression on line $i$ and $\Gamma_{i}$ be the set of all premises and assumptions whose scope includes line $i$. We set out to show "generalized" soundness: if $\Gamma \vdash_{\mathrm{NIL}}^{*} \mathcal{P}$ then $\Gamma \models_{\mathrm{LL}}^{*} \mathcal{P}$. As above, this reduces to the standard result when $\mathcal{P}$ and all the members of $\Gamma$ are formulas with subscript 0 and do not include ' $~ '$. Suppose $\Gamma \vdash_{\mathrm{NIL}}^{*} \mathcal{P}$. Then there is a derivation of $\mathcal{P}$ from premises in $\Gamma$ where $\mathcal{P}$ appears under the scope of the premises alone. By induction on line number of this derivation, we show that for each line $i$ of this derivation, $\Gamma_{i} \models_{L}^{*} \mathcal{P}_{i}$. The case when $\mathcal{P}_{i}=\mathcal{P}$ is the desired result.

Basis: $\mathcal{P}_{1}$ is a premise or an assumption $A_{s} \llbracket$ s.t $\rrbracket$. Then $\Gamma_{1}=\left\{A_{s}\right\} \llbracket\{$ s.t $\} \rrbracket$; so for any $\langle W, R, v\rangle_{m}, v_{m}\left(\Gamma_{1}\right)=1$ iff $v_{m(s)}(A)=1 \llbracket\langle m(s), m(t)\rangle \in R \rrbracket$; so there is no $\langle\mathrm{W}, \mathrm{R}, v\rangle_{\mathrm{m}}$ such that $v_{\mathrm{m}}\left(\Gamma_{1}\right)=1$ but $v_{\mathrm{m}(\mathrm{s})}(\mathrm{A})=0 \llbracket\langle\mathrm{m}(\mathrm{s}), \mathrm{m}(\mathrm{t})\rangle \notin$ $R \rrbracket$. So by VIL*, $\Gamma_{1} \models_{\mathbb{L}}^{*} A_{s} \llbracket s . t \rrbracket$, where this is just to say, $\Gamma_{1} \models_{\mathrm{L}}^{*} \mathcal{P}_{1}$.

Assp: For any $i, 1 \leqslant i<k, \Gamma_{i} \models_{\mathrm{L}}^{*} \mathcal{P}_{i}$.

Show: $\Gamma_{\mathrm{k}} \models_{\mathrm{L}}^{*} \mathcal{P}_{\mathrm{k}}$.

$\mathcal{P}_{k}$ is either a premise, an assumption, or arises from previous lines by $\mathrm{R}, \wedge \mathrm{I}, \wedge \mathrm{E}, \vee \mathrm{I}, \vee \mathrm{E}, \sim \mathrm{I}, \sim \mathrm{E}, \neg \mathrm{I}, \neg \mathrm{E}, \sqsupset \mathrm{I}, \sqsupset \mathrm{E}, \mathrm{AM} \rho, \mathrm{AM} \tau$ or $\mathrm{H}$. If $\mathcal{P}_{\mathrm{k}}$ is a premise or an assumption, then as in the basis, $\Gamma_{k} \models^{*} \mathcal{P}_{k}$. So suppose $\mathcal{P}_{k}$ arises by one of the rules.

(R)

$(\wedge \mathrm{I})$

$(\wedge \mathrm{E})$

$(\mathrm{VE})$

( $\sim$ I) If $\mathcal{P}_{k}$ arises by $\sim \mathrm{I}$, then the picture is like this,

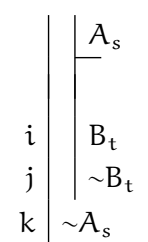

where $i, j<k$ and $\mathcal{P}_{k}$ is $\sim A_{s}$. By assumption, $\Gamma_{i} \models_{L}^{*} B_{t}$ and $\Gamma_{j} \models_{k}^{*}$ $\sim B_{t}$; but by the nature of access, $\Gamma_{i} \subseteq \Gamma_{k} \cup\left\{A_{s}\right\}$ and $\Gamma_{j} \subseteq \Gamma_{k} \cup\left\{A_{s}\right\}$; so by L5.I, $\Gamma_{k} \cup\left\{A_{s}\right\} \models_{\mathrm{L}}^{*} \mathrm{~B}_{\mathrm{t}}$ and $\Gamma_{\mathrm{k}} \cup\left\{A_{s}\right\} \models_{\mathrm{L}}^{*} \sim \mathrm{B}_{\mathrm{t}}$. Suppose $\Gamma_{\mathrm{k}} \not \nvdash_{\mathrm{L}}^{*}$ $\sim A_{s}$; then by VIL*, there is an $I L$ interpretation $\langle W, R, v\rangle_{m}$ such that $v_{\mathrm{m}}\left(\Gamma_{\mathrm{k}}\right)=1$ but $v_{\mathrm{m}(\mathrm{s})}(\sim A)=0$; so by TIL $(\sim), v_{\mathrm{m}(\mathrm{s})}(A)=1$; so $v_{\mathrm{m}}\left(\Gamma_{\mathrm{k}}\right)=$ 1 and $v_{m(s)}(A)=1$; so $v_{m}\left(\Gamma_{k} \cup\left\{A_{s}\right\}\right)=1$; so by VIL*, $v_{m(t)}(B)=1$ and $v_{\mathrm{m}(\mathrm{t})}(\sim \mathrm{B})=1$; from the latter, by $\operatorname{TIL}(\sim), v_{\mathrm{m}(\mathrm{t})}(\mathrm{B})=0$. This is impossible; reject the assumption: $\Gamma_{\mathrm{k}} \models_{\mathrm{L}}^{*} \sim A_{s}$, which is to say, $\Gamma_{\mathrm{k}} \models_{\mathrm{L}}^{*} \mathcal{P}_{\mathrm{k}}$. 
$(\sim \mathrm{E})$

$(\neg \mathrm{I})$ If $\mathcal{P}_{k}$ arises by $\rightarrow \mathrm{I}$, then the picture is like this,<smiles>CC#C[V]</smiles>

where $i<k$, $t$ does not appear in any member of $\Gamma_{k}$ (in any undischarged premise or assumption), and $\mathcal{P}_{k}$ is $\rightarrow A_{s}$. By assumption, $\Gamma_{i} \models_{\mathbb{L}}^{*} \sim A_{t}$; but by the nature of access, $\Gamma_{i} \subseteq \Gamma_{k} \cup\{$ s.t $\}$; so by L5.I, $\Gamma_{k} \cup\{$ s.t $\} \models_{1 L}^{*}$ $\sim A_{t}$. Suppose $\Gamma_{k} \not \not_{\mathrm{L}}^{*} \rightarrow A_{s}$; then by VIL*, there is an $I L$ interpretation $\langle W, R, v\rangle_{m}$ such that $v_{m}\left(\Gamma_{k}\right)=1$ but $v_{m(s)}(\neg A)=0$; so by TIL $(\neg)$, there is some $w \in W$ such that $m(s) R w$ and $v_{w}(A)=1$. Now consider a map $m^{\prime}$ like $m$ except that $m^{\prime}(t)=w$, and consider $\langle W, R, v\rangle_{m^{\prime}}$; since $t$ does not appear in $\Gamma_{k}$, it remains that $v_{m^{\prime}}\left(\Gamma_{k}\right)=1$; and since $m^{\prime}(t)=w$ and $\mathrm{m}^{\prime}(\mathrm{s})=\mathrm{m}(\mathrm{s}),\left\langle\mathrm{m}^{\prime}(\mathrm{s}), \mathrm{m}^{\prime}(\mathrm{t})\right\rangle \in \mathrm{R}$; so $v_{\mathrm{m}^{\prime}}\left(\Gamma_{\mathrm{k}} \cup\{\mathrm{s} . \mathrm{t}\}\right)=1$; so by VIL*, $v_{m^{\prime}(t)}(\sim A)=1$; so by TIL $(\sim), v_{m^{\prime}}(A)=0$. But $m^{\prime}(t)=w$; so $v_{w}(A)=0$. This is impossible; reject the assumption: $\Gamma_{k} \models_{\mathbb{L}}^{*} \rightarrow A_{s}$, which is to say, $\Gamma_{\mathrm{k}} \models_{\mathrm{L}}^{*} \mathcal{P}_{\mathrm{k}}$.

$(\neg \mathrm{E})$ If $\mathcal{P}_{k}$ arises by $\neg \mathrm{E}$, then the picture is like this,

$$
\begin{array}{r|l}
i & \neg A_{s} \\
j & s . t \\
k & \sim A_{t}
\end{array}
$$

where $i, j<k$ and $\mathcal{P}_{k}$ is $\sim A_{t}$. By assumption, $\Gamma_{i} \models_{L}^{*} \neg A_{s}$ and $\Gamma_{j} \models_{\mathrm{L}}^{*}$ s.t; but by the nature of access, $\Gamma_{i} \subseteq \Gamma_{k}$ and $\Gamma_{j} \subseteq \Gamma_{k}$; so by L5.I, $\Gamma_{k} \models_{\mathrm{L}}^{*} \rightarrow A_{s}$ and $\Gamma_{k} \models_{\mathrm{L}}^{*}$ s.t. Suppose $\Gamma_{k} \not \nvdash_{\mathrm{L}}^{*} \sim A_{t}$; then by VIL*, there is some $I L$ interpretation $\langle W, R, v\rangle_{m}$ such that $v_{m}\left(\Gamma_{k}\right)=1$ but $v_{m(t)}(\sim A)=0$; so by TIL $(\sim), v_{m(t)}(A)=1$. Since $v_{m}\left(\Gamma_{k}\right)=1$, by VIL*, $v_{m(s)}(\neg A)=1$ and $\langle\mathrm{m}(\mathrm{s}), \mathrm{m}(\mathrm{t})\rangle \in \mathrm{R}$; from the first of these, by TIL $(\neg)$, any $w$ such that $m(s) R w$ has $v_{w}(A)=0$; so $v_{m(t)}(A)=0$. This is impossible; reject the assumption: $\Gamma_{k} \models_{\mathbb{L}}^{*} A_{t}$, which is to say, $\Gamma_{k} \models_{\mathbb{L}}^{*} \mathcal{P}_{k}$.

（ $\mathrm{I}$ ) If $\mathcal{P}_{k}$ arises by $\sqsupset \mathrm{I}$, then the picture is like this,

$$
\begin{array}{c|l}
i & \mid \begin{array}{l}
s . t \\
A_{t} \\
k
\end{array} \\
B_{t} \\
(A \sqsupset B)_{s}
\end{array}
$$

where $i<k$, $t$ does not appear in any member of $\Gamma_{k}$ (in any undischarged premise or assumption), and $\mathcal{P}_{k}$ is $(A \sqsupset B)_{s}$. By assumption, $\Gamma_{i} \models_{L}^{*}$ 
$B_{t}$; but by the nature of access, $\Gamma_{i} \subseteq \Gamma_{k} \cup\left\{\right.$ s.t, $\left.A_{t}\right\}$; so by L5.I, $\Gamma_{k} \cup$ $\left\{\right.$ s.t. $\left.A_{\mathrm{t}}\right\} \models_{\mathrm{L}}^{*} \mathrm{~B}_{\mathrm{t}}$. Suppose $\Gamma_{\mathrm{k}} \not \forall_{\mathrm{L}}^{*}(A \sqsupset \mathrm{B})_{s}$; then by VIL*, there is an $I L$ interpretation $\langle W, R, v\rangle_{m}$ such that $v_{m}\left(\Gamma_{k}\right)=1$ but $v_{m(s)}(A \sqsupset B)=0$; so by $\operatorname{TIL}(\sqsupset)$, there is some $w \in W$ such that $m(s) R w$ with $v_{w}(A)=1$ and $v_{w}(\mathrm{~B})=0$. Now consider a map $\mathrm{m}^{\prime}$ like $\mathrm{m}$ except that $\mathrm{m}^{\prime}(\mathrm{t})=w$, and consider $\langle W, R, v\rangle_{m^{\prime}}$; since $t$ does not appear in $\Gamma_{k}$, it remains that $v_{m^{\prime}}\left(\Gamma_{k}\right)=1$; since $m^{\prime}(t)=w$ and $m^{\prime}(s)=m(s), v_{m^{\prime}(t)}(A)=1$ and $\left\langle\mathrm{m}^{\prime}(\mathrm{s}), \mathrm{m}^{\prime}(\mathrm{t})\right\rangle \in \mathrm{R}$; so $v_{\mathrm{m}^{\prime}}\left(\Gamma_{\mathrm{k}} \cup\left\{\right.\right.$ s.t. $\left.\left.A_{\mathrm{t}}\right\}\right)=1$; so by VIL*, $v_{\mathrm{m}^{\prime}(\mathrm{t})}(\mathrm{B})=1$. But $\mathrm{m}^{\prime}(\mathrm{t})=w$; so $v_{w}(\mathrm{~B})=1$. This is impossible; reject the assumption: $\Gamma_{\mathrm{k}} \models_{\mathrm{L}}^{*}(A \sqsupset \mathrm{B})_{\mathrm{s}}$, which is to say, $\Gamma_{\mathrm{k}} \models_{\mathrm{L}}^{*} \mathcal{P}_{\mathrm{k}}$.

$(\sqsupset \mathrm{E})$

$(\mathrm{AM} \rho)$

$(\mathrm{AM} \tau)$ If $\mathcal{P}_{k}$ arises by $\mathrm{AM} \tau$, then the picture is like this,
$i \mid s . t$
j t.u
k s.u

where $i, j<k$ and $\mathcal{P}_{k}$ is s.u. By assumption, $\Gamma_{i} \models_{\mathrm{L}}^{*}$ s.t and $\Gamma_{j} \models_{\mathrm{L}}^{*}$ t.u; but by the nature of access, $\Gamma_{\mathrm{i}} \subseteq \Gamma_{\mathrm{k}}$ and $\Gamma_{\mathrm{j}} \subseteq \Gamma_{\mathrm{k}}$; so by L5.I, $\Gamma_{\mathrm{k}} \models_{\mathrm{L}}^{*}$ s.t and $\Gamma_{\mathrm{k}} \models_{\mathrm{L}}^{*}$ t.u. Suppose $\Gamma_{\mathrm{k}} \not \nvdash_{\mathrm{L}}^{*}$ s.u; then by VIL*, there is some $I L$ interpretation $\langle\mathrm{W}, \mathrm{R}, v\rangle_{\mathrm{m}}$ such that $v_{\mathrm{m}}\left(\Gamma_{\mathrm{k}}\right)=1$ but $\langle\mathrm{m}(\mathrm{s}), \mathrm{m}(\mathrm{u})\rangle \notin \mathrm{R}$; since $v_{m}\left(\Gamma_{k}\right)=1$, by VIL*, $\langle m(s), m(t)\rangle \in R$ and $\langle m(t), m(u)\rangle \in R$; but $I L$ includes condition $\tau$; so for any $\langle x, y\rangle,\langle y, z\rangle \in R,\langle x, z\rangle \in R$; so $\langle\mathrm{m}(\mathrm{s}), \mathrm{m}(\mathrm{u})\rangle \in \mathrm{R}$. This is impossible; reject the assumption: $\Gamma_{\mathrm{k}} \models_{\mathrm{L}}^{*} \mathrm{~s} . \mathrm{u}$, which is to say, $\Gamma_{k} \models_{\mathbb{L}}^{*} \mathcal{P}_{k}$.

(H) If $\mathcal{P}_{k}$ arises by $\mathrm{H}$, then the picture is like this,

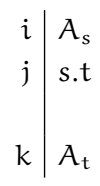

where $i, j<k, A$ has no instance of ' $\sim$ ' and $\mathcal{P}_{k}$ is $A_{t}$. By assumption, $\Gamma_{\mathrm{i}} \models_{\mathrm{L}}^{*} A_{\mathrm{s}}$ and $\Gamma_{\mathrm{j}} \models_{\mathrm{L}}^{*}$ s.t; but by the nature of access, $\Gamma_{\mathrm{i}} \subseteq \Gamma_{\mathrm{k}}$ and $\Gamma_{\mathrm{j}} \subseteq$ $\Gamma_{k}$; so by L5.I, $\Gamma_{k} \models_{\mathrm{L}}^{*} A_{s}$ and $\Gamma_{k} \models_{\mathrm{L}}^{*}$ s.t. Suppose $\Gamma_{k} \not \nvdash_{\mathrm{L}}^{*} A_{t}$; then by VIL*, there is some $I L$ interpretation $\langle\mathrm{W}, \mathrm{R}, v\rangle_{\mathrm{m}}$ such that $v_{\mathrm{m}}\left(\Gamma_{\mathrm{k}}\right)=$ 1 but $v_{m(t)}(A)=0$; since $v_{m}\left(\Gamma_{k}\right)=1$, by VIL*, $v_{m(s)}(A)=1$ and $\langle\mathrm{m}(\mathrm{s}), \mathrm{m}(\mathrm{t})\rangle \in \mathrm{R}$.

Now, by induction on the number of operators in $A$, we show that for A without ' $~ '$ ', if $v_{x}(A)=1$ and $x R y$, then $v_{y}(A)=1$. Suppose $x$ Ry.

Basis: Suppose $A$ is a parameter $p$ and $v_{x}(A)=1$; then $v_{x}(p)=1$; so by condition $h, v_{y}(p)=1$; so $v_{y}(A)=1$. 
Assp: For $0 \leqslant i<k$, if $A$ has $i$ operators and $v_{x}(A)=1$, then $v_{y}(A)=$ 1.

Show: If $A$ has $k$ operators and $v_{x}(A)=1$, then $v_{y}(A)=1$.

If $A$ has $k$ operators and no instance of ' $\sim$ ' then it is of the form, $\mathrm{P} \wedge \mathrm{Q}, \mathrm{P} \vee \mathrm{Q}, \neg \mathrm{P}$, or $\mathrm{P} \sqsupset \mathrm{Q}$, where $\mathrm{P}$ and $\mathrm{Q}$ have $<\mathrm{k}$ operators.

$(\wedge)$ Suppose $A$ is $P \wedge Q$ and $v_{x}(A)=1$; then $v_{x}(P \wedge Q)=1$; so by $\operatorname{TIL}(\wedge), v_{x}(\mathrm{P})=1$ and $v_{x}(\mathrm{Q})=1$; so by assumption, $v_{y}(\mathrm{P})=1$ and $v_{y}(\mathrm{Q})=1$; so by $\operatorname{TIL}(\wedge), v_{y}(\mathrm{P} \wedge \mathrm{Q})=1$; so $v_{y}(A)=1$.

$(\vee)$ Suppose $A$ is $P \vee Q$ and $v_{x}(A)=1$; then $v_{x}(P \vee Q)=1$; so by $\operatorname{TIL}(\mathrm{V}), v_{x}(\mathrm{P})=1$ or $v_{x}(\mathrm{Q})=1$; so by assumption, $v_{y}(\mathrm{P})=1$ or $v_{y}(\mathrm{Q})=1$; so by $\operatorname{TIL}(\mathrm{V}), v_{y}(\mathrm{P} \vee \mathrm{Q})=1$; so $v_{y}(\mathrm{~A})=1$.

$(\rightarrow)$ Suppose $A$ is $\rightarrow P$ and $v_{x}(A)=1$ but $v_{y}(A)=0$; then $v_{x}(\neg P)=1$ but $v_{y}(\neg P)=0$. From the former, by TIL $(\neg)$, any $w$ such that $x R w$ has $v_{w}(P)=0$. From the latter, by TIL $(\neg)$, there is some $z \in W$ such that $y R z$ and $v_{z}(P)=1$. But $x R y$ and $y R z$ so by $\tau$, $x R z$; so $v_{z}(P)=0$. This is impossible; reject the assumption: if $v_{x}(A)=1$, then $v_{y}(A)=1$.

( $\sqsupset)$ Suppose $A$ is $\mathrm{P} \sqsupset \mathrm{Q}$ and $v_{x}(\mathrm{~A})=1$ but $v_{y}(\mathrm{~A})=0$; then $v_{x}(\mathrm{P} \sqsupset$ $\mathrm{Q})=1$ but $v_{y}(\mathrm{P} \sqsupset \mathrm{Q})=0$. From the former, by TIL $(\sqsupset)$, any $w$ such that $x R w$ has $v_{w}(\mathrm{P})=0$ or $v_{w}(\mathrm{Q})=1$. From the latter, by $\operatorname{TIL}(\sqsupset)$, there is some $z \in W$ such that $y \operatorname{Rz}$ where $v_{z}(\mathrm{P})=1$ and $v_{z}(\mathrm{Q})=0$. But $x R y$ and $y R z$ so by $\tau, x R z$; so $v_{z}(\mathrm{P})=0$ or $v_{z}(\mathrm{Q})=1$. This is impossible; reject the assumption: if $v_{x}(\mathrm{~A})=$ 1 , then $v_{y}(A)=1$.

For any such $A$, if $v_{x}(A)=1$, then $v_{y}(A)=1$.

So, returning to the case for $(H), v_{m(t)}(A)=1$. This is impossible; reject the assumption: $\Gamma_{k} \models_{\mathrm{L}}^{*} A_{t}$, which is to say, $\Gamma_{k} \models_{\mathbb{L}}^{*} \mathcal{P}_{k}$.

For any $i, \Gamma_{i} \models_{\mathrm{L}}^{*} \mathcal{P}_{i}$.

THEOREM 5.2 NIL is complete: if $\Gamma \models_{\mathrm{IL}}$ A then $\Gamma \vdash_{\mathrm{NIL}}$ A.

Suppose $\Gamma \models_{\mathrm{IL}} A$; then $\Gamma_{0} \models_{\mathrm{LL}}^{*} A_{0}$; we show that $\Gamma_{0} \vdash_{\mathrm{NIL}}^{*} A_{0}$. Again, this reduces to the standard notion.

Con $\Gamma$ is CONSISTENT iff there is no $A_{s}$ such that $\Gamma \vdash_{\mathrm{NIL}}^{*} A_{s}$ and $\Gamma \vdash_{\mathrm{NIL}}^{*} \sim A_{s}$.

L5.2 If $s$ is 0 or appears in $\Gamma$, and $\Gamma \nvdash_{\mathrm{NIL}}^{*} \sim \mathrm{P}_{\mathrm{s}}$, then $\Gamma \cup\left\{\mathrm{P}_{\mathrm{s}}\right\}$ is consistent.

Suppose $s$ is 0 or appears in $\Gamma$ and $\Gamma \nvdash_{\mathrm{NIL}}^{*} \sim \mathrm{P}_{s}$ but $\Gamma \cup\left\{\mathrm{P}_{\mathrm{s}}\right\}$ is inconsistent. Then there is some $A_{t}$ such that $\Gamma \cup\left\{P_{s}\right\} \vdash_{\mathrm{NIL}}^{*} A_{t}$ and $\Gamma \cup\left\{\mathrm{P}_{s}\right\} \vdash_{\mathrm{NL}}^{*} \sim A_{t}$. But then we can argue, 


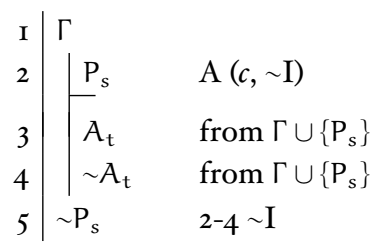

where the assumption is allowed insofar as $s$ is either 0 or appears in $\Gamma$; so $\Gamma \vdash_{\mathrm{NIL}}^{*} \sim \mathrm{P}_{\mathrm{s}}$. But this is impossible; reject the assumption: if $s$ is 0 or introduced in $\Gamma$ and $\Gamma{Y_{\mathrm{NIL}}^{*}}_{\mathrm{P}_{s}}$, then $\Gamma \cup\left\{\mathrm{P}_{\mathrm{s}}\right\}$ is consistent.

L5.3 There is an enumeration of all the subscripted formulas, $\mathcal{P}_{1} \mathcal{P}_{2} \ldots$

Proof by construction as for $\mathrm{L} 2.3$ of $N K \alpha$.

Max $\Gamma$ is S-MAXIMAL iff for any $A_{s}$ either $\Gamma \vdash_{\mathrm{NIL}}^{*} A_{s}$ or $\Gamma \vdash_{\mathrm{NIL}}^{*} \sim A_{s}$.

SGT $\Gamma$ is a SCAPEgOAT set for $\neg$ iff for every formula of the form $\sim \neg A_{s}$, if $\Gamma \vdash_{\mathrm{NIL}}^{*} \sim \longrightarrow A_{s}$ then there is some $t$ such that $\Gamma \vdash_{\mathrm{NIL}}^{*}$ s.t and $\Gamma \vdash_{\mathrm{NIL}}^{*} A_{\mathrm{t}}$.

$\Gamma$ is a SCAPEGOAT set for $\sqsupset$ iff for every formula of the form $\sim(A \sqsupset B)_{s}$, if $\Gamma \vdash_{\mathrm{NIL}}^{*} \sim(A \sqsupset \mathrm{B})_{\mathrm{s}}$ then there is some $t$ such that $\Gamma \vdash_{\mathrm{NIL}}^{*}$ s.t, $\Gamma \vdash_{\mathrm{NIL}}^{*} A_{\mathrm{t}}$ and $\Gamma \vdash_{\mathrm{NIL}}^{*} \sim \mathrm{B}_{\mathrm{t}}$.

$\mathrm{C}\left(\Gamma^{\prime}\right)$ For $\Gamma$ with unsubscripted formulas and the corresponding $\Gamma_{0}$, we construct $\Gamma^{\prime}$ as follows. Set $\Omega_{0}=\Gamma_{0}$. By L5.3, there is an enumeration, $\mathcal{P}_{1}, \mathcal{P}_{2} \ldots$ of all the subscripted formulas; let $\mathcal{E}_{0}$ be this enumeration. Then for the first $A_{s}$ in $\mathcal{E}_{i-1}$ such that $s$ is 0 or included in $\Omega_{i-1}$, let $\mathcal{E}_{i}$ be like $\varepsilon_{i-1}$ but without $A_{s}$, and set,

and

$$
\begin{array}{ll}
\Omega_{i}=\Omega_{i-1} & \text { if } \Omega_{i-1} \vdash_{\mathrm{NIL}}^{*} \sim A_{s} \\
\Omega_{i^{*}}=\Omega_{i-1} \cup\left\{A_{s}\right\} & \text { if } \Omega_{i-1} \nvdash_{\mathrm{NIL}}^{*} \sim A_{s}
\end{array}
$$

$$
\Omega_{i}=\Omega_{i^{*}} \quad \text { if } A_{s} \text { is not of the form } \sim P_{s} \text { or } \sim(P \sqsupset
$$

$\mathrm{Q})_{s}$

$\Omega_{i}=\Omega_{i^{*}} \cup\left\{\right.$ s.t, $\left.P_{t}\right\} \quad$ if $A_{s}$ is of the form $\sim P_{s}$

$\Omega_{i}=\Omega_{i^{*}} \cup\left\{\right.$ s.t, $\left.P_{t}, \sim Q_{t}\right\} \quad$ if $A_{s}$ is of the form $\sim(P \sqsupset Q)_{s}$

-where $t$ is the first subscript not included in $\Omega_{i *}$.

then

$$
\Gamma^{\prime}=\bigcup_{i \geqslant 0} \Omega_{i}
$$

Note that there is always sure to be a subscript $t$ not in $\Omega_{i^{*}}$ insofar as there are infinitely many subscripts, and at any stage only finitely many formulas are added - the only subscripts in the initial $\Omega_{0}$ being 0 . Suppose $s$ is introduced in $\Gamma^{\prime}$; then there is some $\Omega_{i}$ in which it is first introduced; and any formula $\mathcal{P}_{j}$ in the original enumeration that has subscript $s$ is sure to be "considered" for inclusion at a subsequent stage.

L5.4 For any s included in $\Gamma^{\prime}, \Gamma^{\prime}$ is s-maximal. 
Suppose $s$ is included in $\Gamma^{\prime}$ but $\Gamma^{\prime}$ is not s-maximal. Then there is some $A_{s}$ such that $\Gamma^{\prime} \nvdash_{\mathrm{NL}}^{*} A_{s}$ and $\Gamma^{\prime} \nvdash_{\mathrm{NIL}}^{*} \sim A_{s}$. For any $i$, each member of $\Omega_{i-1}$ is in $\Gamma^{\prime}$; so if $\Omega_{i-1} \vdash_{\mathrm{NIL}}^{*} \sim A_{s}$ then $\Gamma^{\prime} \vdash_{\mathrm{NIL}}^{*} \sim A_{s}$; but $\Gamma^{\prime} \nvdash_{\mathrm{NIL}}^{*} \sim A_{s}$; so $\Omega_{i-1} \nvdash_{\mathrm{NIL}}^{*} \sim A_{s}$; so since $s$ is included in $\Gamma^{\prime}$, there is a stage in the construction that sets $\Omega_{i^{*}}=\Omega_{i-1} \cup\left\{A_{s}\right\}$; so by construction, $A_{s} \in \Gamma^{\prime}$; so $\Gamma^{\prime} \vdash_{\mathrm{NIL}}^{*} A_{s}$. This is impossible; reject the assumption: $\Gamma^{\prime}$ is s-maximal.

L5.5 If $\Gamma_{0}$ is consistent, then each $\Omega_{i}$ is consistent.

Suppose $\Gamma_{0}$ is consistent.

Basis: $\Omega_{0}=\Gamma_{0}$ and $\Gamma_{0}$ is consistent; so $\Omega_{0}$ is consistent.

Assp: For any $i, 0 \leqslant i<k, \Omega_{i}$ is consistent.

Show: $\Omega_{k}$ is consistent.

$\Omega_{\mathrm{k}}$ is either (i) $\Omega_{\mathrm{k}-1}$, or (ii) $\Omega_{\mathrm{k}^{*}}=\Omega_{\mathrm{k}-1} \cup\left\{\mathrm{A}_{\mathrm{s}}\right\}$, (iii) $\Omega_{\mathrm{k}^{*}} \cup\left\{\right.$ s.t, $\left.\mathrm{P}_{\mathrm{t}}\right\}$ or (iv) $\Omega_{k^{*}} \cup\left\{\right.$ s.t, $\left.P_{t}, \sim Q_{t}\right\}$.

(i) Suppose $\Omega_{k}$ is $\Omega_{k-1}$. By assumption, $\Omega_{k-1}$ is consistent; so $\Omega_{k}$ is consistent.

(ii) Suppose $\Omega_{k}$ is $\Omega_{k^{*}}=\Omega_{k-1} \cup\left\{A_{s}\right\}$. Then by construction, $s$ is 0 or in $\Omega_{k-1}$ and $\Omega_{k-1} \nvdash_{\mathrm{NIL}}^{*} \sim A_{s}$; so by L5.2, $\Omega_{k-1} \cup\left\{A_{s}\right\}$ is consistent; so $\Omega_{k}$ is consistent.

(iii) Suppose $\Omega_{k}$ is $\Omega_{k^{*}} \cup\left\{\right.$ s.t, $\left.P_{t}\right\}$. In this case, as above, $\Omega_{k *}$ is consistent and by construction, $\sim \mathrm{P}_{\mathrm{s}} \in \Omega_{\mathrm{k}^{*}}$. Suppose $\Omega_{\mathrm{k}}$ is inconsistent. Then there are $A_{\mathfrak{u}}$ and $\sim A_{\mathfrak{u}}$ such that $\Omega_{\mathrm{k}^{*}} \cup\left\{\right.$ s.t, $\left.P_{t}\right\} \vdash_{\text {NIL }}^{*}$ $A_{\mathfrak{u}}$ and $\Omega_{k^{*}} \cup\left\{\right.$ s.t. $\left.P_{t}\right\} \vdash_{\mathrm{NIL}}^{*} \sim A_{\mathfrak{u}}$. So reason as follows,

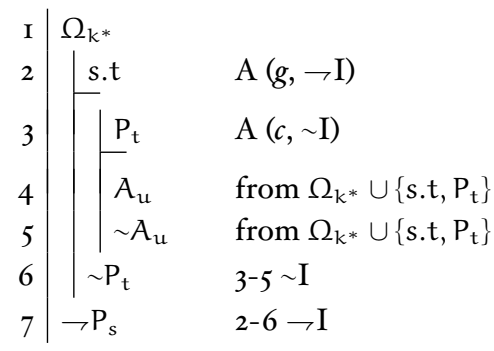

where, by construction, $t$ is not in $\Omega_{\mathrm{k}^{*}}$. So $\Omega_{\mathrm{k}^{*}} \vdash_{\mathrm{NIL}}^{*} \rightarrow \mathrm{P}_{\mathrm{s}}$; but $\sim \mathrm{P}_{\mathrm{s}} \in \Omega_{\mathrm{k}^{*}}$; so $\Omega_{\mathrm{k}^{*}} \vdash_{\mathrm{NIL}}^{*} \sim \mathrm{P}_{\mathrm{s}}$; so $\Omega_{\mathrm{k}^{*}}$ is inconsistent. This is impossible; reject the assumption: $\Omega_{k}$ is consistent.

(iv) Suppose $\Omega_{k}$ is $\Omega_{k^{*}} \cup\left\{\right.$ s.t, $\left.P_{t}, \sim Q_{t}\right\}$. In this case, as above, $\Omega_{k *}$ is consistent and by construction, $\sim(P \sqsupset Q)_{s} \in \Omega_{k^{*}}$. Suppose $\Omega_{k}$ is inconsistent. Then there are $A_{\mathfrak{u}}$ and $\sim A_{\mathfrak{u}}$ such that $\Omega_{k^{*}} \cup$ $\left\{\right.$ s.t, $\left.P_{t}, \sim Q_{t}\right\} \vdash_{\mathrm{NIL}}^{*} A_{\mathfrak{u}}$ and $\Omega_{\mathrm{k}^{*}} \cup\left\{\right.$ s.t. $\left.\mathrm{P}_{\mathrm{t}}, \sim \mathrm{Q}_{\mathrm{t}}\right\} \vdash_{\mathrm{NIL}}^{*} \sim A_{\mathrm{u}}$. So reason as follows, 


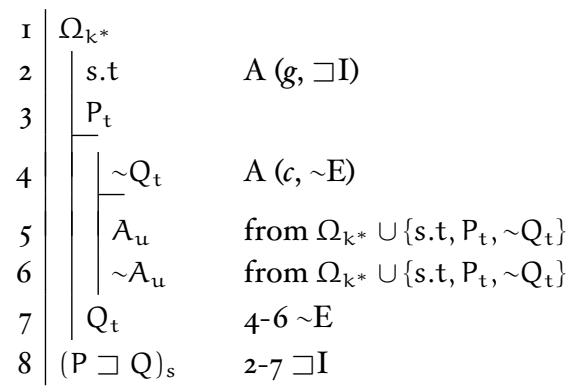

where, by construction, $t$ is not in $\Omega_{\mathrm{k}^{*}}$. So $\Omega_{\mathrm{k}^{*}} \vdash_{\mathrm{NIL}}^{*}(\mathrm{P} \sqsupset \mathrm{Q})_{s}$; but $\sim(\mathrm{P} \sqsupset \mathrm{Q})_{\mathrm{s}} \in \Omega_{\mathrm{k}^{*}}$; so $\Omega_{\mathrm{k}^{*}} \vdash_{\mathrm{NIL}}^{*} \sim(\mathrm{P} \sqsupset \mathrm{Q})_{\mathrm{s}}$; so $\Omega_{\mathrm{k}^{*}}$ is inconsistent. This is impossible; reject the assumption: $\Omega_{k}$ is consistent.

For any $i, \Omega_{i}$ is consistent.

L5.6 If $\Gamma_{0}$ is consistent, then $\Gamma^{\prime}$ is consistent.

Reasoning parallel to $\mathrm{L} 2.6$ for $N K \alpha$.

L5.7 If $\Gamma_{0}$ is consistent, then $\Gamma^{\prime}$ is a scapegoat set for $\neg$ and $\sqsupset$.

For $\neg$. Suppose $\Gamma_{0}$ is consistent and $\Gamma^{\prime} \vdash_{\mathrm{NII}}^{*} \sim \neg \mathrm{P}_{\mathrm{s}}$. By L 5.6, $\Gamma^{\prime}$ is consistent; and by the constraints on subscripts, $s$ is included in $\Gamma^{\prime}$. Since $\Gamma^{\prime}$ is consistent, $\Gamma^{\prime} H_{\mathrm{NIL}}^{*} \sim \sim \mathrm{P}_{\mathrm{s}}$; so there is a stage in the construction process where $\Omega_{i^{*}}=\Omega_{i-1} \cup\left\{\sim \neg P_{s}\right\}$ and $\Omega_{i}=\Omega_{i^{*}} \cup\left\{\right.$ s.t, $\left.P_{t}\right\}$; so by construction, s.t $\in \Gamma^{\prime}$ and $\mathrm{P}_{\mathrm{t}} \in \Gamma^{\prime}$; so $\Gamma^{\prime} \vdash_{\mathrm{NIL}}^{*}$ s.t and $\Gamma^{\prime} \vdash_{\mathrm{NIL}}^{*} \mathrm{P}_{\mathrm{t}}$. So $\Gamma^{\prime}$ is a scapegoat set for $\neg$.

For $\sqsupset$. Suppose $\Gamma_{0}$ is consistent and $\Gamma^{\prime} \vdash_{\mathrm{NIL}}^{*} \sim(\mathrm{P} \sqsupset \mathrm{Q})_{s}$. By L5.6, $\Gamma^{\prime}$ is consistent; and by the constraints on subscripts, $s$ is included in $\Gamma^{\prime}$. Since $\Gamma^{\prime}$ is consistent, $\Gamma^{\prime} \nvdash_{\mathrm{NIL}}^{*} \sim \sim(\mathrm{P} \sqsupset \mathrm{Q})_{s}$; so there is a stage in the construction process where $\Omega_{i^{*}}=\Omega_{i-1} \cup\left\{\sim(\mathrm{P} \sqsupset \mathrm{Q})_{s}\right\}$ and $\Omega_{i}=\Omega_{i^{*}} \cup$ $\left\{\right.$ s.t, $\left.P_{t}, \sim Q_{t}\right\}$; so by construction, s.t $\in \Gamma^{\prime}, P_{t} \in \Gamma^{\prime}$ and $\sim Q_{t} \in \Gamma^{\prime}$; so $\Gamma^{\prime} \vdash_{\mathrm{NIL}}^{*}$ s.t, $\Gamma^{\prime} \vdash_{\mathrm{NIL}}^{*} \mathrm{P}_{\mathrm{t}}$ and $\Gamma^{\prime} \vdash_{\mathrm{NIL}}^{*} \sim \mathrm{Q}_{\mathrm{t}}$. So $\Gamma^{\prime}$ is a scapegoat set for $\sqsupset$.

$\mathrm{C}$ (I) We construct an interpretation $\mathrm{I}=\langle\mathrm{W}, \mathrm{R}, v\rangle$ based on $\Gamma^{\prime}$ as follows. Let $W$ have a member $w_{s}$ corresponding to each subscript $s$ included in $\Gamma^{\prime}$. Then set $\left\langle w_{s}, w_{t}\right\rangle \in R$ iff $\Gamma^{\prime} \vdash_{\mathrm{NIL}}^{*}$ s.t, and $v_{w_{s}}(p)=1$ iff $\Gamma^{\prime} \vdash_{\mathrm{NIL}}^{*} p_{s}$.

L5.8 If $\Gamma_{0}$ is consistent then for $\langle W, R, v\rangle$ constructed as above, and for any $s$ included in $\Gamma^{\prime}, v_{w_{s}}(A)=1$ iff $\Gamma^{\prime} \vdash_{\mathrm{NIL}}^{*} A_{s}$.

Suppose $\Gamma_{0}$ is consistent and $s$ is included in $\Gamma^{\prime}$. By L 5.4, $\Gamma^{\prime}$ is s-maximal. By L 5.6 and $\mathrm{L}_{5.7}, \Gamma^{\prime}$ is consistent and a scapegoat set for $\rightarrow$ and $\sqsupset$. Now by induction on the number of operators in $A_{s}$,

Basis: If $A_{s}$ has no operators, then it is a parameter $p_{s}$ and by construction, $v_{w_{s}}(p)=1 \mathrm{iff} \Gamma^{\prime} \vdash_{\mathrm{NIL}}^{*} p_{s}$. So $v_{w_{s}}(A)=1 \mathrm{iff} \Gamma^{\prime} \vdash_{\mathrm{NIL}}^{*} A_{s}$. 
Assp: For any $i, 0 \leqslant i<k$, if $A_{s}$ has $i$ operators, then $v_{w_{s}}(A)=1$ iff $\Gamma^{\prime} \vdash_{\mathrm{NIL}}^{*} A_{\mathrm{s}}$.

Show: If $A_{s}$ has $k$ operators, then $\nu_{w_{s}}(A)=1$ iff $\Gamma^{\prime} \vdash_{\mathrm{NIL}}^{*} A_{s}$.

If $A_{s}$ has $k$ operators, then it is of the form $\sim P_{s},(P \wedge Q)_{s},(P \vee$ $\mathrm{Q})_{s},(\mathrm{P} \sqsupset \mathrm{Q})_{s}$, or $\rightarrow \mathrm{P}_{s}$ where $\mathrm{P}$ and $\mathrm{Q}$ have $<\mathrm{k}$ operators.

( ) $A_{s}$ is $\sim P_{s}$. (i) Suppose $v_{w_{s}}(A)=1$; then $v_{w_{s}}(\sim P)=1$; so by $\operatorname{TIL}(\sim), v_{w_{s}}(\mathrm{P})=0$; so by assumption, $\Gamma^{\prime}{H_{\mathrm{NIL}}^{*}}^{*} \mathrm{P}_{s}$; so by $s^{-}$ maximality, $\Gamma^{\prime} \vdash_{\mathrm{NIL}}^{*} \sim \mathrm{P}_{s}$, where this is to say, $\Gamma^{\prime} \vdash_{\mathrm{NIL}}^{*} A_{s}$. (ii) Suppose $\Gamma^{\prime} \vdash_{\mathrm{NIL}}^{*} A_{s}$; then $\Gamma^{\prime} \vdash_{\mathrm{NIL}}^{*} \sim \mathrm{P}_{s}$; so by consistency, $\Gamma^{\prime} \vdash_{\mathrm{NIL}}^{*} \mathrm{P}_{s}$; so by assumption, $v_{w_{s}}(\mathrm{P})=0$; so by TIL $(\sim), v_{w_{s}}(\sim \mathrm{P})=1$, where this is to say, $v_{w_{s}}(A)=1$. So $v_{w_{s}}(A)=1$ iff $\Gamma^{\prime} \vdash_{\mathrm{NIL}}^{*} A_{s}$.

$(\wedge)$

$(\mathrm{V})$

$(\rightarrow) A_{s}$ is $\neg P_{s}$. (i) Suppose $v_{w_{s}}(A)=1$ but $\Gamma^{\prime} \nvdash_{\mathrm{NIL}}^{*} A_{s}$; then $v_{w_{s}}(\neg P)=$ 1 but $\Gamma^{\prime} H_{\mathrm{NIL}}^{*} \rightarrow \mathrm{P}_{s}$. From the latter, by $s$-maximality, $\Gamma^{\prime} \vdash_{\mathrm{NIL}}^{*}$ $\sim \mathrm{P}_{s}$; so, since $\Gamma^{\prime}$ is a scapegoat set for $\neg$, there is some $t$ such that $\Gamma^{\prime} \vdash_{\mathrm{NIL}}^{*}$ s.t and $\Gamma^{\prime} \vdash_{\mathrm{NIL}}^{*} \mathrm{P}_{\mathrm{t}}$; from the first, by construction, $\left\langle w_{s}, w_{t}\right\rangle \in R$; and from the second, by assumption, $v_{w_{t}}(\mathrm{P})=1$; so by $\operatorname{TIL}(\neg), v_{w_{s}}(\neg \mathrm{P})=0$. This is impossible; reject the assumption: if $v_{w_{s}}(A)=1$, then $\Gamma^{\prime} \vdash_{\mathrm{NIL}}^{*} A_{s}$.

(ii) Suppose $\Gamma^{\prime} \vdash_{\mathrm{NIL}}^{*} A_{s}$ but $v_{w_{s}}(A)=0$; then $\Gamma^{\prime} \vdash_{\mathrm{NIL}}^{*} \rightarrow \mathrm{P}_{\mathrm{s}}$ but $v_{w_{s}}(\neg \mathrm{P})=0$. From the latter, by TIL $(\neg)$, there is some $w_{t} \in$ $W$ such that $w_{s} R w_{t}$ and $v_{w_{t}}(\mathrm{P})=1$; so by assumption, $\Gamma^{\prime} \vdash_{\mathrm{NII}}^{*} \mathrm{P}_{\mathrm{t}}$; but since $w_{\mathrm{s}} \mathrm{R} w_{\mathrm{t}}$, by construction, $\Gamma^{\prime} \vdash_{\mathrm{NIL}}^{*}$ s.t; so by $(\neg \mathrm{E}), \Gamma^{\prime} \vdash_{\mathrm{NIL}}^{*}$ $\sim \mathrm{P}_{\mathrm{t}}$; so by consistency, $\Gamma^{\prime} H_{\mathrm{NIL}}^{*} \mathrm{P}_{\mathrm{t}}$. This is impossible; reject the assumption: if $\Gamma^{\prime} \vdash_{\mathrm{NIL}} A_{s}$ then $v_{w_{s}}(A)=1$. So $v_{w_{s}}(A)=1$ iff $\Gamma^{\prime} \vdash_{\mathrm{NIL}}^{*} A_{\mathrm{s}}$.

For any $A_{s}, v_{w_{s}}(A)=1$ iff $\Gamma^{\prime} \vdash_{\mathrm{NIL}}^{*} A_{s}$.

L5.9 If $\Gamma_{0}$ is consistent, then $\langle\mathrm{W}, \mathrm{R}, v\rangle$ constructed as above is an $I L$ interpretation.

For this, we need to show that the interpretation meets the $\rho, \tau$ and $h$ conditions.

( $\rho$ ) Suppose $w_{s} \in W$. Then by construction, $s$ is a subscript in $\Gamma^{\prime}$; so by $(\mathrm{AM} \rho), \Gamma^{\prime} \vdash_{\mathrm{NIL}}^{*}$ s.s; so by construction, $\left\langle w_{s}, w_{\mathrm{s}}\right\rangle \in \mathrm{R}$ and $\rho$ is satisfied.

$(\tau)$ 
(b) Suppose $v_{w_{s}}(p)=1$ and $w_{s} R w_{t}$. Then by construction, $\Gamma^{\prime} \vdash_{\mathrm{NIL}}^{*}$ $\mathrm{p}_{\mathrm{s}}$ and $\Gamma^{\prime} \vdash_{\mathrm{NIL}}^{*}$ s.t; so by $(\mathrm{H}), \Gamma^{\prime} \vdash_{\mathrm{NIL}}^{*} p_{\mathrm{t}}$; so by construction, $v_{w_{\mathrm{t}}}(\mathrm{p})=1$.

MAP For any $w_{s} \in W$, set $m(s)=w_{s}$; otherwise $m(s)$ is arbitrary.

L5.IO If $\Gamma_{0}$ is consistent, then $v_{m}\left(\Gamma_{0}\right)=1$.

Reasoning parallel to L2. Io for $N K \alpha$.

Main result: Suppose $\Gamma \models_{\mathrm{IL}}$ A but $\Gamma \nvdash_{\mathrm{NIL}}$ A. Then $\Gamma_{0} \models_{\mathrm{IL}}^{*} A_{0}$ but $\Gamma_{0} \nvdash_{\mathrm{NIL}}^{*} A_{0}$. By a simple derivation, if $\Gamma_{0} \vdash_{\mathrm{NIL}}^{*} \sim \sim A_{0}$, then $\Gamma_{0} \vdash_{\mathrm{NIL}}^{*} A_{0}$; so $\Gamma_{0} \nvdash_{\mathrm{NIL}}^{*} \sim \sim A_{0}$; so by L5.2, $\Gamma_{0} \cup\left\{\sim A_{0}\right\}$ is consistent; so by $L_{5.9}$ and $L_{5} . \mathrm{IO}$, there is an $I L$ interpretation $\langle W, R, v\rangle_{m}$ constructed as above such that $v_{m}\left(\Gamma_{0} \cup\left\{\sim A_{0}\right\}\right)=1$; so $v_{m}(0)(\sim A)=$ 1 ; so by $\operatorname{TIL}(\sim), v_{\mathfrak{m}(0)}(A)=0$; so $v_{\mathfrak{m}}\left(\Gamma_{0}\right)=1$ and $v_{\mathfrak{m}(0)}(A)=0$; so by VIL*, $\Gamma_{0} \not \nvdash_{\mathrm{LL}}^{*} A_{0}$. This is impossible; reject the assumption: if $\Gamma \models_{\mathrm{IL}} A$, then $\Gamma \vdash_{\mathrm{NIL}} A$.

\section{MANY-VAlued logics: $M x(\mathrm{CH} .7,8)$}

This section develops derivations for the systems for which Priest supplies tableaux in his text: (classical logic), $K_{3}, L P$ and FDE. Thus there are no derivations for his semantically described $L_{3}$ and $R M_{3}$.

\section{I LANGUAGE / SEMANTIC NOTIONS}

LMx The LANGUAGE consists of propositional parameters $p_{0}, p_{1} \ldots$ with the operators, $\neg, \wedge, \vee$, and $\supset$. Each propositional parameter is a FORMULA; if $A$ and $B$ are formulas, so are $\neg A,(A \wedge B),(A \vee B)$, and $(A \supset B) . A \equiv B$ abbreviates $(A \supset B) \wedge(B \supset A)$.

IMx An interpretation is a function $v$ which assigns to each propositional parameter some subset of $\{0,1\}$; so $v(p)$ is $\phi,\{1\},\{0\}$ or $\{1,0\}$. Intuitively, $v(p)$ is true iff $1 \in v(p)$ and $v(p)$ is false iff $0 \in v(p)$. Where $x$ is empty or includes some combination of the following constraints,

$$
\begin{array}{lll}
\text { exc } & \text { for no } p \text { are both } 0 \in v(p) \text { and } 1 \in v(p) & \text { exclusion } \\
\text { exh } & \text { for any } p \text {, either } 1 \in v(p) \text { or } 0 \in v(p) & \text { exhaustion }
\end{array}
$$

$v$ is an $M x$ interpretation when it meets the constraints from $x . M_{C L}$ has both exc and exh, $M_{K_{3}}$ just exc, $M_{L P}$ just exh, and $M_{F D}$ neither exc nor exh (these are classical logic, and Priest's $K_{3}, L P$ and $F D E$ ).

TM For complex expressions,

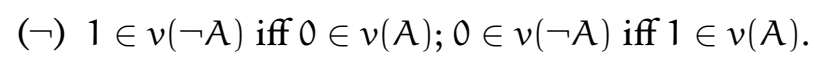

$(\wedge) 1 \in v(A \wedge B)$ iff $1 \in v(A)$ and $1 \in v(B) ; 0 \in v(A \wedge B)$ iff $0 \in v(A)$ or $0 \in v(B)$. 
( $\vee) \quad 1 \in v(A \vee B)$ iff $1 \in v(A)$ or $1 \in v(B) ; 0 \in v(A \vee B)$ iff $0 \in v(A)$ and $0 \in v(B)$.

(つ) $1 \in v(\mathrm{~A} \supset \mathrm{B})$ iff $0 \in v(\mathrm{~A})$ or $1 \in v(\mathrm{~B}) ; 0 \in v(\mathrm{~A} \supset \mathrm{B})$ iff $1 \in v(\mathrm{~A})$ and $0 \in v(B)$.

For a set $\Gamma$ of formulas, $1 \in v(\Gamma)$ iff $1 \in v(A)$ for each $A \in \Gamma$; then,

$\mathrm{VMx} \Gamma \models_{M x} A$ iff there is no $M x$ interpretation $v$ such that $1 \in v(\Gamma)$ but $1 \notin v(A)$.

This account is adequate to the (superficially) different presentations in these chapters of Priest. For the multivalued approach: classical logic has values $\{0\},\{1\}$, with $\{1\}$ designated; $K_{3}$ has $\phi,\{0\},\{1\}$, with $\{1\}$ designated; $L P$ has $\{0\}$, $\{1\},\{0,1\}$, with $\{1\}$ and $\{0,1\}$ designated; and $F D E$ has $\phi,\{0\},\{1\},\{0,1\}$, with $\{1\}$ and $\{0,1\}$ designated. For the relational approach, we identify the relation as set membership. And a $v$ as above maps to a Routley interpretation with $v_{w}(p)=1$ iff $1 \in v(p)$, and $v_{w^{*}}(p)=0$ iff $0 \in v(p) .5$ Then, in each case, conditions for truth and validity are as above.

\subsection{NATURAL DERIVATIONS: NMx}

Introduce expressions of the sort $A$ and $\bar{A}$. Intuitively $\bar{A}$ indicates that $A$ is not false. Let $\backslash A \backslash$ and $/ A /$ represent either $A$ or $\bar{A}$ where what is represented is constant in a given context, but $\backslash A \backslash$ and / $A$ / are opposite. And similarly for $\| A / /$ and $\backslash A \|$, though there need be no fixed relation between overlines on $\backslash A \backslash$ and $\backslash A \|$. Except for a pair of new rules corresponding to conditions exc and $e x h$, derivation rules mirror ones for classical logic. $(\equiv \mathrm{I})$ and $(\equiv \mathrm{E})$ are now derived.

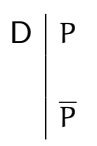

$\mathrm{R} \mid \begin{aligned} & / \mathrm{P} / \\ & / \mathrm{P} /\end{aligned}$

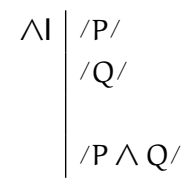

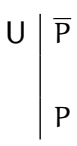

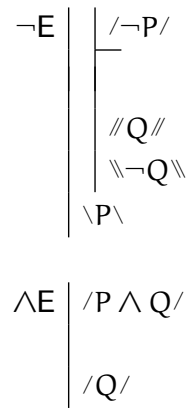

${ }^{5}$ For this, see [4, sections 8.5.8, 8.7.I7 and 8.7.I8] along with L6.o below. 

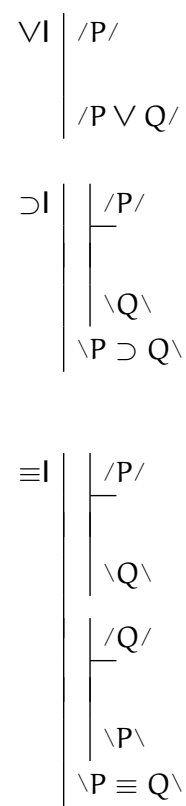
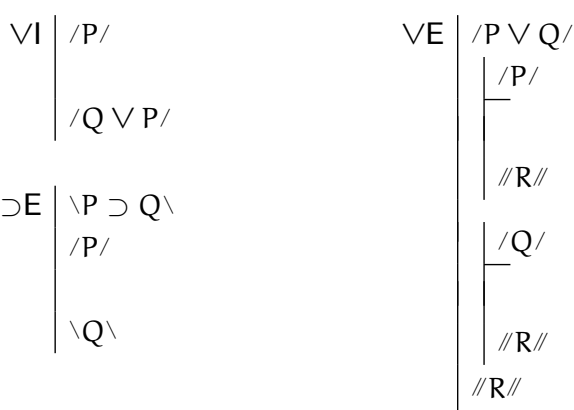

$\equiv \mathrm{E} \mid \begin{aligned} & \backslash \mathrm{P} \equiv \mathrm{Q} \\ & / \mathrm{P} / \\ & \backslash \mathrm{Q} \backslash\end{aligned}$

$N M C L$ has all the rules. $N M_{K_{3}}$ has the I- and E-rules for $\neg, \wedge, \vee, \supset$ with (R) and (D) (for truth down). NMLP has the I- and E-rules for $\neg, \wedge, \vee, \supset$ with (R) and (U) (for truth $u p$ ). NMFD has just the I- and E-rules for $\neg, \wedge, \vee, \supset$ with (R). Where the members of $\Gamma$ and $A$ are expressions without overlines,

$N M x \Gamma \vdash_{N M x} A$ iff there is an $N M x$ derivation of $A$ from the members of $\Gamma$.

Two-way derived rules carry over from $C L$ with consistent overlines. Thus, e.g.,

Impl $/ \mathrm{P} \supset \mathrm{Q} / \triangleleft \triangleright / \neg \mathrm{P} \vee \mathrm{Q} /$

$/ \neg \mathrm{P} \supset \mathrm{Q}^{\prime} \triangleleft \triangleright / \mathrm{P} \vee \mathrm{Q} /$

MT, NB and DS appear in the forms,

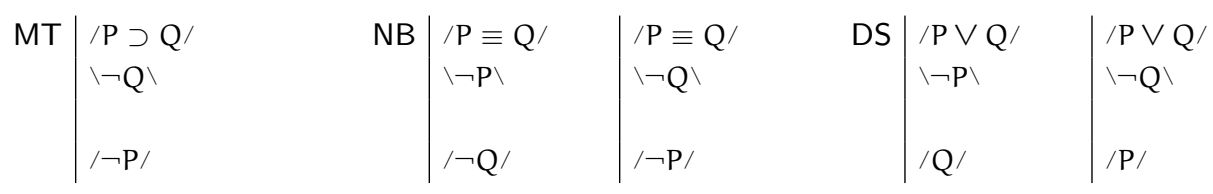

As examples, here are derivations, cast to show the general forms, for MT and the second form of DS. 


\begin{tabular}{|c|c|c|}
\hline I & $/ \mathrm{P} \supset \mathrm{Q}^{\prime}$ & $\mathrm{P}$ \\
\hline 2 & $\backslash \neg \mathrm{Q} \backslash$ & $\mathrm{P}$ \\
\hline 3 & $\backslash P \backslash$ & $\mathrm{A}(c, \neg \mathrm{I})$ \\
\hline 4 & /Q/ & $\mathrm{I}, 3 \supset \mathrm{E}$ \\
\hline 5 & $\backslash \neg \mathrm{Q} \backslash$ & $2 \mathrm{R}$ \\
\hline 6 & $/ \neg \mathrm{P} /$ & $3-5 \neg \mathrm{I}$ \\
\hline
\end{tabular}

\begin{tabular}{|c|c|c|}
\hline \multicolumn{3}{|c|}{$\left(\mathrm{P} \vee \mathrm{Q} /, \backslash \neg \mathrm{Q} \backslash \vdash_{\mathrm{NM} x} / \mathrm{P} /\right.$} \\
\hline I & $/ \mathrm{P} \vee \mathrm{Q} /$ & $\mathrm{P}$ \\
\hline 2 & $\backslash \neg Q \backslash$ & $\mathrm{P}$ \\
\hline 3 & $/ \mathrm{P} /$ & $\mathrm{A}(g, \mathrm{I} \vee \mathrm{E})$ \\
\hline 4 & $/ \mathrm{P} /$ & $3 \mathrm{R}$ \\
\hline 5 & /Q/ & $\mathrm{A}(g, \mathrm{I} \vee \mathrm{E})$ \\
\hline 6 & $\backslash \neg \mathrm{P} \backslash$ & $\mathrm{A}(c, \neg \mathrm{E})$ \\
\hline 7 & $/ Q^{\prime}$ & $5 \mathrm{R}$ \\
\hline 8 & $\backslash \neg \mathrm{Q} \backslash$ & $2 \mathrm{R}$ \\
\hline 9 & $/ \mathrm{P} /$ & $6-8 \neg \mathrm{E}$ \\
\hline O & $/ \mathrm{P} /$ & $\mathrm{I}, 3-4,5-9 \vee \mathrm{E}$ \\
\hline
\end{tabular}

And for some particular results requiring (D) and (U), here are demonstrations of standard rule and axioms for classical logic, making use of the full rule set (see, e.g. [12, chapter 3]).

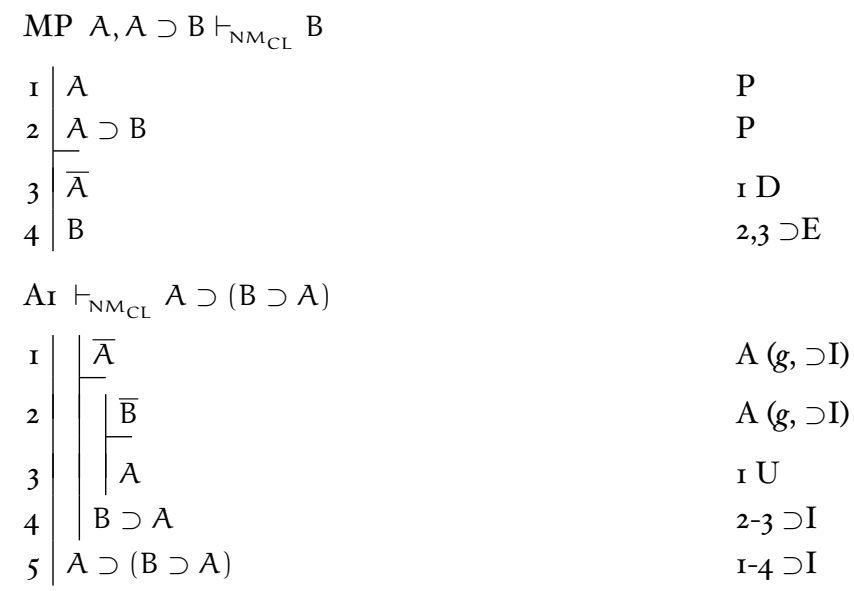

$\mathrm{A} 2 \vdash_{\mathrm{NM} C L}[\mathrm{~A} \supset(\mathrm{B} \supset \mathrm{C})] \supset[(\mathrm{A} \supset \mathrm{B}) \supset(\mathrm{A} \supset \mathrm{C})]$

\begin{tabular}{|c|c|c|}
\hline I & $\bar{A} \supset(\mathrm{B} \supset \mathrm{C})$ & $\mathrm{A}(g, \supset \mathrm{I})$ \\
\hline 2 & $\overline{A \supset B}$ & $\mathrm{~A}(g, \supset \mathrm{I})$ \\
\hline 3 & $\bar{A}$ & $\mathrm{~A}(g, \supset \mathrm{I})$ \\
\hline 4 & $A \supset B$ & $2 \mathrm{U}$ \\
\hline 5 & B & $3,4 \supset \mathrm{E}$ \\
\hline 6 & $A \supset(B \supset C)$ & I $U$ \\
\hline 7 & $B \supset C$ & 3,6 つE \\
\hline 8 & $\overline{\mathrm{B}}$ & $5 \mathrm{D}$ \\
\hline & C & $7,8 \supset \mathrm{E}$ \\
\hline & $A \supset C$ & 3-9 つI \\
\hline II & $(A \supset B) \supset(A \supset C)$ & 2-IO つI \\
\hline $\mathrm{I} 2$ & $A \supset(B \supset C)] \supset[(A \supset B) \supset(A \supset C)]$ & I-II $\supset$ I \\
\hline
\end{tabular}






Of course, there is not much point going back-and-forth between overline and non-overline expressions in the full classical system. But these examples should illustrate the rules. And overlines matter for the other systems.

\subsection{SOUNDNESS AND COMPLETENESS}

Preliminaries: Begin with generalized notions of truth and validity to include expressions with overlines. First, holding as a generalization of TM. Say / A/ bolds iff $h(A)=1$ and otherwise fails. As usual, for the following, cases omitted are like ones worked, and so left to the reader.

HM (B) $h(p)=1$ iff $1 \in v(p)$, and otherwise $h(p)=0 ; h(\bar{p})=1$ iff $0 \notin v(p)$, and otherwise $h(\bar{p})=0$.

$(\neg) h(/ \neg A /)=1$ iff $h(\backslash A \backslash)=0$, and otherwise $h(/ \neg A /)=0$.

$(\wedge) h(/ A \wedge B /)=1$ iff $h(/ A /)=1$ and $h(/ B /)=1$, and otherwise $h(/ A \wedge B /)=0$.

(V) $h(/ A \vee B /)=1$ iff $h(/ A /)=1$ or $h(/ B /)=1$, and otherwise $h(/ A \vee B /)=0$.

(つ) $h(/ A \supset B /)=1$ iff $h(\backslash A \backslash)=0$ or $h(/ B /)=1$, and otherwise $h(/ A \supset B /)=0$.

This formulation nicely mirrors the original classical definition TCL. And $\mathrm{h}$ and $v$ are related as one would expect.

L6.0 For any $M x$ interpretation $v$ and corresponding $h, h(A)=1$ iff $1 \in v(A)$, and $h(\bar{A})=1$ iff $0 \notin v(A)$.

Basis: If $A$ has no operators, then it is a parameter $p$. $\operatorname{By} \operatorname{HM}(B), h(p)=$ 1 iff $1 \in v(p)$ and $h(\bar{p})=1$ iff $0 \notin v(p) ;$ so $h(A)=1$ iff $1 \in v(A)$, and $h(\bar{A})=1$ iff $0 \notin v(A)$.

Assp: For $0 \leqslant i<k$, if $A$ has $i$ operators, then $h(A)=1$ iff $1 \in v(A)$, and $h(\bar{A})=1$ iff $0 \notin v(A)$. 
Show: If $A$ has $k$ operators, then $h(A)=1$ iff $1 \in v(A)$, and $h(\bar{A})=1$ iff $0 \notin v(A)$.

If $A$ has $k$ operators, then it is of the form, $\neg P, P \wedge Q, P \vee Q$, or $\mathrm{P} \supset \mathrm{Q}$ where $\mathrm{P}$ and $\mathrm{Q}$ have $<\mathrm{k}$ operators.

$(\neg)$ Suppose $A$ is $\neg$ P. By HM( $(\neg), h(\neg P)=1$ iff $h(\bar{P})=0$; by assumption, iff $0 \in v(\mathrm{P})$; by $\mathrm{TM}(\neg)$ iff $1 \in v(\neg \mathrm{P})$. By $\operatorname{HM}(\neg), h(\overline{\neg P})=1$ iff $h(\mathrm{P})=0$; by assumption, iff $1 \notin v(\mathrm{P})$; by $\mathrm{TM}(\neg)$ iff $0 \notin v(\neg \mathrm{P})$. So $h(A)=1$ iff $1 \in v(A)$, and $h(\bar{A})=1$ iff $0 \notin v(A)$.

$(\wedge)$ Suppose $A$ is $P \wedge Q$. By $\operatorname{HM}(\wedge), h(P \wedge Q)=1$ iff $h(P)=1$ and $h(\mathrm{Q})=1$; by assumption, iff $1 \in v(\mathrm{P})$ and $1 \in v(\mathrm{Q})$; by $\mathrm{TM}(\wedge)$ iff $1 \in v(\mathrm{P} \wedge \mathrm{Q})$. By $\mathrm{HM}(\wedge), h(\overline{\mathrm{P} \wedge \mathrm{Q}})=1$ iff $h(\overline{\mathrm{P}})=1$ and $h(\bar{Q})=1$; by assumption, iff $0 \notin v(\mathrm{P})$ and $0 \notin v(\mathrm{Q})$; by TM $(\wedge)$ iff $0 \notin v(P \wedge Q)$. So $h(A)=1$ iff $1 \in v(A)$, and $h(\bar{A})=1$ iff $0 \notin v(A)$.

$(\mathrm{V})$

(つ) Suppose $A$ is $P \supset Q$. By $H M(\supset), h(P \supset Q)=1$ iff $h(\bar{P})=0$ or $h(\mathrm{Q})=1$; by assumption, iff $0 \in v(\mathrm{P})$ or $1 \in v(\mathrm{Q})$; by $\mathrm{TM}(\supset)$ iff $1 \in v(\mathrm{P} \supset \mathrm{Q})$. By $\mathrm{HM}(\supset), \mathrm{h}(\overline{\mathrm{P} \supset \mathrm{Q}})=1$ iff $h(\mathrm{P})=0$ or $\mathrm{h}(\overline{\mathrm{Q}})=1$; by assumption, iff $1 \notin v(\mathrm{P})$ or $0 \notin v(\mathrm{Q})$; by $\mathrm{TM}(\supset)$ iff $0 \notin v(P \supset Q)$. So $h(A)=1$ iff $1 \in v(A)$, and $h(\bar{A})=1$ iff $0 \notin v(A)$.

For any $A, h(A)=1$ iff $1 \in v(A)$, and $h(\bar{A})=1$ iff $0 \notin v(A)$.

So $A$ holds iff $1 \in v(A)$, and otherwise fails; and $\bar{A}$ holds iff $0 \notin v(A)$, and otherwise fails. This permits natural generalizations for notions of validity. For any $v$, where $\Gamma$ is a set of expressions with or without overlines, say $h(\Gamma)=1$ iff $h(/ A /)=1$ for each $/ A / \in \Gamma$. Then,

$\mathrm{VMx}^{*} \Gamma \models_{M x}^{*} / \mathrm{A} /$ iff there is no $M x$ interpretation $v$ and corresponding $\mathrm{h}$ such that $h(\Gamma)=1$ but $h(/ A /)=0$.

$\mathrm{NMx}^{*} \Gamma \vdash_{\mathrm{NMx}}^{*} / \mathrm{A} /$ iff there is an $N M x$ derivation of $/ \mathrm{A} /$ from the members of $\Gamma$.

These notions reduce to the standard ones when all the members of $\Gamma$ and $/ A /$ are without overlines. This is obvious for $\mathrm{NMx}^{*}$. And similarly, we have $h(A)=1$ iff $1 \in v(A)$; so $V M x^{*}$ collapses to $V M x$.

THEOREM 6.I NMx is sound: If $\Gamma \vdash_{N M x}$ A then $\Gamma \vdash_{M x} A$.

L6.I If $\Gamma \subseteq \Gamma^{\prime}$ and $\Gamma \models_{M x}^{*} / \mathrm{P} /$, then $\Gamma^{\prime} \models_{M x}^{*} / \mathrm{P} /$.

Suppose $\Gamma \subseteq \Gamma^{\prime}$ and $\Gamma \models_{M x}^{*} / \mathrm{P} /$, but $\Gamma^{\prime} \nvdash_{M x}^{*} / \mathrm{P} /$. From the latter, by $\mathrm{VMx}^{*}$, there is some $v$ and $h$ such that $h\left(\Gamma^{\prime}\right)=1$ but $h(/ \mathrm{P} /)=0$. But 
since $h\left(\Gamma^{\prime}\right)=1$ and $\Gamma \subseteq \Gamma^{\prime}, h(\Gamma)=1$; so $h(\Gamma)=1$ but $h(/ \mathrm{P} /)=0$; so by $\mathrm{VMx}^{*}, \Gamma \not \nvdash_{\mathrm{Mx}}^{*} / \mathrm{P} /$. This is impossible; reject the assumption: if $\Gamma \subseteq \Gamma^{\prime}$ and $\Gamma \models_{M x}^{*} / \mathrm{P} /$, then $\Gamma^{\prime} \models_{M x}^{*} / \mathrm{P} /$.

Main result: For each line in a derivation let $\mathcal{P}_{i}$ be the formula on line $i$ (with or without overlines) and set $\Gamma_{i}$ equal to the set of all premises and assumptions whose scope includes line $i$. We set out to show "generalized" soundness: if $\Gamma \vdash_{\mathrm{NMx}}^{*} / \mathrm{A} /$ then $\Gamma \models_{\mathrm{Mx}}^{*} / \mathrm{A} /$. As above, this reduces to the standard result when the members of $\Gamma$ and $A$ are without overlines. Suppose $\Gamma \vdash_{N M x}^{*} / A /$. Then there is a derivation of $/ A$ / from premises in $\Gamma$ where $/ A$ / appears under the scope of the premises alone. By induction on line number of this derivation, we show that for each line $i$ of this derivation, $\Gamma_{i} \models_{M x}^{*} \mathcal{P}_{i}$. The case when $\mathcal{P}_{i}=/ \mathrm{A} /$ is the desired result.

Basis: $\mathcal{P}_{1}$ is a premise or an assumption $/ \mathrm{A} /$. Then $\Gamma_{1}=\{/ \mathrm{A} /\}$; $\operatorname{so} h\left(\Gamma_{1}\right)=1 \mathrm{iff}$ $h(/ A /)=1$; so there is no $h$ such that $h\left(\Gamma_{1}\right)=1$ but $h(/ A /)=0$. So by $\mathrm{VMx}^{*}, \Gamma_{1} \models_{M x}^{*} / \mathrm{A} /$, where this is just to say, $\Gamma_{1} \models_{M x}^{*} \mathcal{P}_{1}$.

Assp: For any $i, 1 \leqslant i<k, \Gamma_{i} \models_{\text {Mx }}^{*} \mathcal{P}_{i}$.

Show: $\Gamma_{\mathrm{k}} \models_{M x}^{*} \mathcal{P}_{\mathrm{k}}$.

$\mathcal{P}_{k}$ is either a premise, an assumption, or arises from previous lines by $\mathrm{R}, \supset \mathrm{I}, \supset \mathrm{E}, \wedge \mathrm{I}, \wedge \mathrm{E}, \neg \mathrm{I}, \neg \mathrm{E}, \vee \mathrm{I}, \vee \mathrm{E}$ or, depending on the system, D or $\mathrm{U}$. If $\mathcal{P}_{k}$ is a premise or an assumption, then as in the basis, $\Gamma_{k} \models_{\text {Mx }}^{*} \mathcal{P}_{k}$. So suppose $\mathcal{P}_{k}$ arises by one of the rules.

(R)

( $\supset$ I) If $\mathcal{P}_{k}$ arises by $\supset \mathrm{I}$, then the picture is like this,

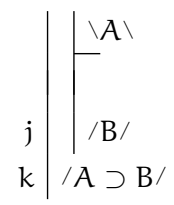

where $j<k$ and $\mathcal{P}_{k}$ is $/ A \supset B /$. By assumption, $\Gamma_{j} \models_{M x}^{*} / B /$; and by the nature of access, $\Gamma_{j} \subseteq \Gamma_{k} \cup\{\backslash A \backslash\}$; so by L6.I, $\Gamma_{k} \cup\{\backslash A \backslash\} \models_{M x}^{*} / B /$. Suppose $\Gamma_{k} \not \nvdash_{M x}^{*} / A \supset B /$; then by VMx* , there is some $v$ and $h$ such that $h\left(\Gamma_{k}\right)=$ 1 but $h(/ A \supset B /)=0$; from the latter, by $\operatorname{HM}(\supset), h(\backslash A \backslash)=1$ and $h(/ B /)=0$; so $h\left(\Gamma_{k}\right)=1$ and $h(\backslash A \backslash)=1$; so $h\left(\Gamma_{k} \cup\{\backslash A \backslash\}\right)=1$; so by $\mathrm{VMx}^{*}, \mathrm{~h}(/ \mathrm{B} /)=1$. This is impossible; reject the assumption: $\Gamma_{\mathrm{k}} \models_{\mathrm{Mx}}^{*}$ $/ \mathrm{A} \supset \mathrm{B} /$, which is to say, $\Gamma_{\mathrm{k}} \models_{\mathrm{Mx}}^{*} \mathcal{P}_{\mathrm{k}}$.

( $\supset \mathrm{E})$ If $\mathcal{P}_{k}$ arises by $\supset \mathrm{E}$, then the picture is like this,

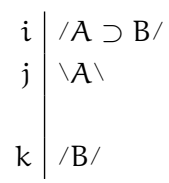


where $i, j<k$ and $\mathcal{P}_{k}$ is $/ \mathrm{B} /$. By assumption, $\Gamma_{i} \models_{M x}^{*} / A \supset B /$ and $\Gamma_{j} \models_{M x}^{*} \backslash A \backslash$; but by the nature of access, $\Gamma_{i} \subseteq \Gamma_{k}$ and $\Gamma_{j} \subseteq \Gamma_{k}$; so by L6.I, $\Gamma_{k} \models_{M x}^{*} / A \supset B /$ and $\Gamma_{k} \models_{M x}^{*} \backslash A \backslash$. Suppose $\Gamma_{k} \not \nvdash_{M x}^{*} / B /$; then by $\mathrm{VMx}^{*}$, there is some $v$ and $\mathrm{h}$ such that $\mathrm{h}\left(\Gamma_{\mathrm{k}}\right)=1 \mathrm{but} \mathrm{h}(/ \mathrm{B} /)=0$; since $h\left(\Gamma_{k}\right)=1$, by $V M x^{*}, h(/ A \supset B /)=1$ and $h(\backslash A \backslash)=1$; from the former, by $\mathrm{HM}(\supset), h(\backslash A \backslash)=0$ or $h(/ B /)=1$; so $h(/ B /)=1$. This is impossible; reject the assumption: $\Gamma_{\mathrm{k}} \models_{\mathrm{Mx}}^{*} / \mathrm{B} /$, which is to say, $\Gamma_{\mathrm{k}} \models_{\mathrm{Mx}}^{*} \mathcal{P}_{\mathrm{k}}$.

$(\wedge \mathrm{I})$

$(\wedge \mathrm{E})$

$(\neg \mathrm{I})$ If $\mathcal{P}_{k}$ arises by $\neg \mathrm{I}$, then the picture is like this,

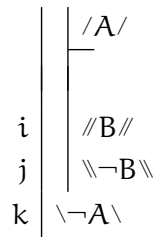

where $i, j<k$ and $\mathcal{P}_{k}$ is $\backslash \neg A \backslash$. By assumption, $\Gamma_{i} \models_{M x}^{*} / / B / /$ and $\Gamma_{j} \models_{M x}^{*}$ $\| \neg B \Downarrow$; but by the nature of access, $\Gamma_{i} \subseteq \Gamma_{k} \cup\{/ A /\}$ and $\Gamma_{j} \subseteq \Gamma_{k} \cup\{/ A /\}$; so by L6.I, $\Gamma_{k} \cup\{/ A /\} \models_{M x}^{*} / / B / /$ and $\Gamma_{k} \cup\{/ A /\} \models_{M x}^{*}\|\neg B\|$. Suppose $\Gamma_{\mathrm{k}} \not \nvdash_{\mathrm{Mx}}^{*} \backslash \neg A \backslash$; then by $\mathrm{VMx}$, there is some $v$ and $h$ such that $h\left(\Gamma_{\mathrm{k}}\right)=1$ but $h(\backslash \neg A \backslash)=0$; from the latter, by $\operatorname{HM}(\neg), h(/ A /)=1$; so $h\left(\Gamma_{k}\right)=$ 1 and $h(/ A /)=1$; so $h\left(\Gamma_{k} \cup\{/ A /\}\right)=1$; so by $V M x^{*}, h(/ / B / /)=1$ and $h(\|\neg B\|)=1$; from the latter, by $\operatorname{HM}(\neg), h(/ / B / /)=0$. This is impossible; reject the assumption: $\Gamma_{\mathrm{k}} \models_{\mathrm{Mx}}^{*} \backslash \neg A \backslash$, which is to say, $\Gamma_{\mathrm{k}} \models_{\mathrm{Mx}_{\mathrm{x}}}^{*}$ $\mathcal{P}_{k}$.

(D) If $\mathcal{P}_{k}$ arises by $\mathrm{D}$, then the picture is like this,

$$
\begin{array}{l|l}
i & A \\
k & \bar{A}
\end{array}
$$

where $i<k$ and $\mathcal{P}_{k}$ is $\bar{A}$. Where this rule is included in $N M x, M x$ has condition exc, so no interpretation has $v(p)=\{1,0\}$. By assumption, $\Gamma_{\mathrm{i}} \models_{M x}^{*} A$; but by the nature of access, $\Gamma_{\mathrm{i}} \subseteq \Gamma_{\mathrm{k}}$; so by L6.I, $\Gamma_{\mathrm{k}} \models_{\mathrm{Mx}_{\mathrm{x}}}^{*} A$. Suppose $\Gamma_{k} \nvdash_{M x}^{*} \bar{A}$; then by $\mathrm{VMx}^{*}$, there is some $v$ and $h$ such that $h\left(\Gamma_{k}\right)=1$ but $h(\bar{A})=0$; since $h\left(\Gamma_{k}\right)=1$, by VMx*, $h(A)=1$. But for these interpretations, for any $A$, if $h(A)=1$ then $h(\bar{A})=1$. 
Basis: $A$ is a parameter $p$. Suppose $h(A)=1$; then $h(p)=1$; so by $\operatorname{HM}(B), 1 \in v(p)$; so by exc, $0 \notin v(p)$; so by $\operatorname{HM}(B), h(\bar{p})=1$; so $h(\bar{A})=1$.

Assp: For any $i, 0 \leqslant i<k$, if $A$ has $i$ operators, and $h(A)=1$, then $h(\bar{A})=1$.

Show: If $A$ has $k$ operators, and $h(A)=1$, then $h(\bar{A})=1$.

If $A$ has $k$ operators, then $A$ is of the form, $\neg P, P \wedge Q, P \vee Q$, or $\mathrm{P} \supset \mathrm{Q}$, where $\mathrm{P}$ and $\mathrm{Q}$ have $<\mathrm{k}$ operators.

$(\neg) A$ is $\neg P$. Suppose $h(A)=1$; then $h(\neg P)=1$; so by $\operatorname{HM}(\neg)$, $h(\bar{P})=0$; so by assumption, $h(P)=0$; so by $\operatorname{HM}(\neg), h(\overline{\neg P})=1$, which is to say, $h(\bar{A})=1$.

$(\wedge) A$ is $P \wedge Q$. Suppose $h(A)=1$; then $h(P \wedge Q)=1$; so by $H M(\wedge)$, $h(P)=1$ and $h(Q)=1$; so by assumption, $h(\bar{P})=1$ and $h(\bar{Q})=$ 1; so by $H M(\wedge), h(\overline{P \wedge Q})=1$, which is to say $h(\bar{A})=1$.

$(\mathrm{V})$

(つ) $A$ is $P \supset$ Q. Suppose $h(A)=1$; then $h(P \supset Q)=1$; so by $\operatorname{HM}(\supset), h(\bar{P})=0$ or $h(Q)=1$; so by assumption, $h(P)=0$ or $h(\bar{Q})=1$; so by $\operatorname{HM}(\supset), h(\overline{P \supset Q})=1$, which is to say $h(\bar{A})=1$.

For any $A$, if $h(A)=1$, then $h(\bar{A})=1$.

So, returning to the case for $(D), h(\bar{A})=1$. This is impossible; reject the assumption: $\Gamma_{k} \models_{M x}^{*} \bar{A}$, which is to say, $\Gamma_{k} \models_{M x}^{*} \mathcal{P}_{k}$.

(U) If $\mathcal{P}_{\mathrm{k}}$ arises by $\mathrm{U}$, then the picture is like this,

$$
\begin{array}{l|l}
i & \bar{A} \\
k & A
\end{array}
$$

where $i<k$ and $\mathcal{P}_{k}$ is $A$. Where this rule is included in $N M x, M x$ has condition $e x h$, so no interpretation has $v(p)=\phi$. By assumption, $\Gamma_{\mathrm{i}} \models_{M x}^{*} \bar{A}$; but by the nature of access, $\Gamma_{\mathrm{i}} \subseteq \Gamma_{\mathrm{k}}$; so by L6.I, $\Gamma_{\mathrm{k}} \models_{M x}^{*} \bar{A}$. Suppose $\Gamma_{k} \nvdash_{M x}^{*} A$; then by $V_{M x}^{*}$, there is some $v$ and $h$ such that $h\left(\Gamma_{k}\right)=1$ but $h(A)=0$; since $h\left(\Gamma_{k}\right)=1$, by VMx* $h(\bar{A})=1$. But for these interpretations, for any $A$, if $h(\bar{A})=1$ then $h(A)=1$.

Basis: $A$ is a parameter $p$. Suppose $h(\bar{A})=1$; then $h(\bar{p})=1$; so by $\operatorname{HM}(\mathrm{B}), 0 \notin v(\mathrm{p})$; so by $e x h, 1 \in v(\mathrm{p})$; so by $\operatorname{HM}(\mathrm{B}), \mathrm{h}(\mathrm{p})=1$; so $h(A)=1$.

Assp: For any $i, 0 \leqslant i<k$, if $A$ has $i$ operators, and $h(\bar{A})=1$, then $h(A)=1$.

Show: If $A$ has $k$ operators, and $h(\bar{A})=1$, then $h(A)=1$.

If $A$ has $k$ operators, then $A$ is of the form, $\neg P, P \wedge Q, P \vee Q$, or $\mathrm{P} \supset \mathrm{Q}$, where $\mathrm{P}$ and $\mathrm{Q}$ have $<k$ operators. 
$(\neg) A$ is $\neg$ P. Suppose $h(\bar{A})=1$; then $h(\overline{\neg P})=1$; so by $\operatorname{HM}(\neg)$, $h(P)=0$; so by assumption, $h(\bar{P})=0$; so by $\operatorname{HM}(\neg), h(\neg P)=1$, which is to say, $h(A)=1$.

$(\wedge) A$ is $P \wedge Q$. Suppose $h(\bar{A})=1$; then $h(\overline{P \wedge Q})=1$; so by $H M(\wedge)$, $h(\bar{P})=1$ and $h(\bar{Q})=1$; so by assumption, $h(P)=1$ and $h(Q)=$ 1 ; so by $\operatorname{HM}(\wedge), h(P \wedge Q)=1$, which is to say $h(A)=1$.

$(\mathrm{V})$

(つ) $A$ is $P \supset$ Q. Suppose $h(\bar{A})=1$; then $h(\overline{P \supset Q})=1$; so by $H M(\supset)$, $h(P)=0$ or $h(\bar{Q})=1$; so by assumption, $h(\bar{P})=0$ or $h(Q)=1$; so by $\operatorname{HM}(\supset), h(P \supset Q)=1$, which is to say $h(A)=1$.

For any $A$, if $h(\bar{A})=1$, then $h(A)=1$.

So, returning to the case for $(U), h(A)=1$. This is impossible; reject the assumption: $\Gamma_{\mathrm{k}} \models_{M x}^{*} A$, which is to say, $\Gamma_{\mathrm{k}} \models_{\mathrm{Mx}}^{*} \mathcal{P}_{\mathrm{k}}$.

For any $i, \Gamma_{i} \models_{M x}^{*} A_{i}$.

THEOREM 6.2 NMx is complete: if $\Gamma \models_{\mathrm{Mx}}$ A then $\Gamma \vdash_{\mathrm{NMx}}$ A.

Suppose $\Gamma \models_{M x} A$; then $\Gamma \models_{M x}^{*} A$; we show that $\Gamma \vdash_{N M x}^{*} A$. Again, this reduces to the standard notion when there are no overlines. Fix on some particular constraint(s) $x$. Then definitions of consistency etc. are relative to it.

Con $\Gamma$ is CONSISTENT iff there is no $A$ such that $\Gamma \vdash_{\mathrm{NMx}}^{*} / A /$ and $\Gamma \vdash_{\mathrm{NMx}}^{*} \backslash \neg A \backslash$.

L6.2 If $\Gamma \nvdash_{\mathrm{NMx}}^{*} \backslash \neg \mathrm{P} \backslash$, then $\Gamma \cup\{/ \mathrm{P} /\}$ is consistent.

Suppose $\Gamma \nvdash_{N M x}^{*} \backslash \neg \mathrm{P} \backslash$ but $\Gamma \cup\{/ \mathrm{P} /\}$ is inconsistent. Then there is some A such that $\Gamma \cup\{/ \mathrm{P} /\} \vdash_{\text {NMx }}^{*} / / \mathrm{A} / /$ and $\Gamma \cup\{/ \mathrm{P} /\} \vdash_{\mathrm{NM}}^{*}\|\neg A\|$. But then we can argue,

$$
\begin{array}{l|ll}
\mathrm{I} & \Gamma & \\
2 & \mid / \mathrm{P} / & \mathrm{A}(c, \neg \mathrm{I}) \\
& \mid / \mathrm{A} / / & \text { from } \Gamma \cup\{/ \mathrm{P} /\} \\
4 & \|\neg A\| & \text { from } \Gamma \cup\{/ \mathrm{P} /\} \\
5 & \backslash \neg \mathrm{P} \backslash & 2-4 \neg \mathrm{I}
\end{array}
$$

So $\Gamma \vdash_{\mathrm{NMx}}^{*} \backslash \neg \mathrm{P} \backslash$. But this is impossible; reject the assumption: if $\Gamma \nvdash_{\mathrm{NMx}}^{*}$ $\backslash \neg \mathrm{P} \backslash$, then $\Gamma \cup\{/ \mathrm{P} /\}$ is consistent.

L6.3 There is an enumeration of all the formulas, $\mathcal{P}_{1}, \mathcal{P}_{2} \ldots$

Proof by construction. A simple approach is to order $A_{1}, A_{2} \ldots$ in the usual way, and let the final enumeration be, $A_{1}, \bar{A}_{1}, A_{2}, \bar{A}_{2} \ldots$

Max $\Gamma$ is Maximal iff for any $A$ either $\Gamma \vdash_{\mathrm{NMx}}^{*} / A /$ or $\Gamma \vdash_{\mathrm{NMx}}^{*} \backslash \neg A \backslash$. 
$\mathrm{C}\left(\Gamma^{\prime}\right)$ We construct a $\Gamma^{\prime}$ from $\Gamma$ as follows. Set $\Omega_{0}=\Gamma$. By L6.3, there is an enumeration, $\mathcal{P}_{1}, \mathcal{P}_{2} \ldots$ of all the formulas; for any $\mathcal{P}_{i}=/ \mathrm{A} /$ in this series set,

then

$$
\begin{array}{ll}
\Omega_{i}=\Omega_{i-1} & \text { if } \Omega_{i-1} \vdash_{N M x}^{*} \backslash \neg A \backslash \\
\Omega_{i}=\Omega_{i-1} \cup\{/ A /\} & \text { if } \Omega_{i-1} \nvdash_{N M x}^{*} \backslash \neg A \backslash
\end{array}
$$

$$
\Gamma^{\prime}=\bigcup_{i \geqslant 0} \Omega_{i}
$$

L6.4 $\Gamma^{\prime}$ is maximal.

Suppose $\Gamma^{\prime}$ is not maximal. Then there is some $\mathcal{P}_{i}=/ \mathrm{A} /$ such that $\Gamma^{\prime} \nvdash_{N M x}^{*} / A /$ and $\Gamma^{\prime} \nvdash_{N M x}^{*} \backslash \neg A \backslash$. For any $i$, each member of $\Omega_{i-1}$ is in $\Gamma^{\prime}$; so if $\Omega_{i-1} \vdash_{\mathrm{NMx}}^{*} \backslash \neg A \backslash$ then $\Gamma^{\prime} \vdash_{\mathrm{NMx}}^{*} \backslash \neg A \backslash$; but $\Gamma^{\prime} \nvdash_{\mathrm{NMx}}^{*} \backslash \neg A \backslash$; so $\Omega_{i-1} \nvdash_{\mathrm{NMx}}^{*} \backslash \neg A \backslash$; so by construction, $\Omega_{i}=\Omega_{i-1} \cup\{/ A / \gamma$; so by construction, /A / $\in \Gamma^{\prime}$; so $\Gamma^{\prime} \vdash_{\mathrm{NMx}}^{*} / \mathrm{A} /$. This is impossible; reject the assumption: $\Gamma^{\prime}$ is maximal.

L6.5 If $\Gamma$ is consistent, then each $\Omega_{i}$ is consistent.

Suppose $\Gamma$ is consistent.

Basis: $\Omega_{0}=\Gamma$ and $\Gamma$ is consistent; so $\Omega_{0}$ is consistent.

Assp: For any $i, 0 \leqslant i<k, \Omega_{i}$ is consistent.

Show: $\Omega_{k}$ is consistent.

$\Omega_{k}$ is either $\Omega_{k-1}$ or $\Omega_{k-1} \cup\{/ A /\}$. Suppose the former; by assumption, $\Omega_{k-1}$ is consistent; so $\Omega_{k}$ is consistent. Suppose the latter; then by construction, $\Omega_{\mathrm{k}-1} \nvdash_{\mathrm{NMx}}^{*} \backslash \neg \mathrm{A} \backslash$; so by L6.2, $\Omega_{\mathrm{k}-1} \cup\{/ \mathrm{A} /\}$ is consistent; so $\Omega_{\mathrm{k}}$ is consistent.

For any $i, \Omega_{i}$ is consistent.

L6.6 If $\Gamma$ is consistent, then $\Gamma^{\prime}$ is consistent.

Suppose $\Gamma$ is consistent, but $\Gamma^{\prime}$ is not; from the latter, there is some $\mathrm{P}$ such that $\Gamma^{\prime} \vdash_{\mathrm{NMx}}^{*} / \mathrm{P} /$ and $\Gamma^{\prime} \vdash_{\mathrm{NMx}}^{*} \backslash \neg \mathrm{P} \backslash$. Consider derivations Di and $\mathrm{D}_{2}$ of these results and the premises of these derivations. Where $\mathcal{P}_{i}$ is the last of these premises in the enumeration of formulas, by the construction of $\Gamma^{\prime}$, each of the premises must be a member of $\Omega_{i}$; so Di and D2 are derivations from $\Omega_{i}$; so $\Omega_{i}$ is not consistent. But since $\Gamma$ is consistent, by L6.5, $\Omega_{i}$ is consistent. This is impossible; reject the assumption: if $\Gamma$ is consistent then $\Gamma^{\prime}$ is consistent.

$\mathrm{C}(v)$ We construct an interpretation $v$ based on $\Gamma^{\prime}$ as follows. For any parameter $p$, set $1 \in v(p)$ iff $\Gamma^{\prime} \vdash_{\mathrm{NMx}}^{*} p$, and $0 \in v(p)$ iff $\Gamma^{\prime} \vdash_{\mathrm{NMx}}^{*} \bar{p}$.

L6.7 If $\Gamma$ is consistent then for any $A, h(/ A /)=1 \mathrm{iff} \Gamma^{\prime} \vdash_{\text {NMx }}^{*} / A /$.

Suppose $\Gamma$ is consistent. By L6.4, $\Gamma^{\prime}$ is maximal; by L6.6, $\Gamma^{\prime}$ is consistent. Now by induction on the number of operators in $A$, 
Basis: If $A$ has no operators, then it is a parameter $p$ or $\bar{p}$. By construction, $\Gamma^{\prime} \vdash_{\mathrm{NMx}}^{*} p$ iff $1 \in v(p)$; by $\operatorname{HM}(\mathrm{B})$, iff $h(p)=1$. Similarly, by construction, $\Gamma^{\prime} \nvdash_{\mathrm{NMx}}^{*} \overline{\mathrm{p}}$ iff $0 \in v(\mathrm{p})$; by $\operatorname{HM}(\mathrm{B})$, iff $h(\bar{p}) \neq 1$. So $h(/ p /)=1$ iff $\Gamma^{\prime} \vdash_{N M x}^{*} / p /$, which is to say, $h(/ A /)=1$ iff $\Gamma^{\prime} \vdash_{\mathrm{NMx}}^{*} / \mathrm{A} /$.

Assp: For any $i, 0 \leqslant i<k$, if $A$ has $i$ operators, then $h(/ A /)=1$ iff $\Gamma^{\prime} \vdash_{\mathrm{NMx}}^{*} / A /$.

Show: If $A$ has $k$ operators, then $h(/ A /)=1$ iff $\Gamma^{\prime} \vdash_{\mathrm{NMx}}^{*} / A /$.

If $A$ has $k$ operators, then it is of the form $\neg P, P \wedge Q, P \vee Q$ or $\mathrm{P} \supset \mathrm{Q}$ where $\mathrm{P}$ and $\mathrm{Q}$ have $<k$ operators.

$(\neg) A$ is $\neg P$. (i) Suppose $h(/ A /)=1$; then $h(/ \neg P /)=1$; so by $\operatorname{HM}(\neg), h(\backslash P \backslash)=0$; so by assumption, $\Gamma^{\prime} \nvdash_{N M x}^{*} \backslash P \backslash$; so by maximality, $\Gamma^{\prime} \vdash_{\mathrm{NMx}}^{*} / \neg \mathrm{P} /$, where this is to say, $\Gamma^{\prime} \vdash_{\mathrm{NMx}}^{*} / \mathrm{A} /$. (ii) Suppose $\Gamma^{\prime} \vdash_{\mathrm{NMx}}^{*} / \mathrm{A} /$; then $\Gamma^{\prime} \vdash_{\mathrm{NMx}}^{*} / \neg \mathrm{P} /$; so by consistency, $\Gamma^{\prime} \vdash_{\mathrm{NMx}}^{*} \backslash \mathrm{P} \backslash$; so by assumption, $h(\backslash P \backslash)=0$; so by $\operatorname{HM}(\neg), h(/ \neg P /)=1$, where this is to say, $h(/ A /)=1$. So $h(/ A /)=1 \mathrm{iff} \Gamma^{\prime} \vdash_{\text {NMx }}^{*} / A /$.

$(\wedge)$

$(\mathrm{V})$

(つ) A is $P \supset$ Q. (i) Suppose $h(/ A /)=1$ but $\Gamma^{\prime} H_{N M x}^{*} / A /$; then $\mathrm{h}(/ \mathrm{P} \supset \mathrm{Q} /)=1$ but $\Gamma^{\prime} H_{\mathrm{NMx}}^{*} / \mathrm{P} \supset \mathrm{Q} /$. From the latter, by maximality, $\Gamma^{\prime} \vdash_{\mathrm{NMx}}^{*} \backslash \neg(\mathrm{P} \supset \mathrm{Q}) \backslash$; from this it follows, by the following derivations,

\begin{tabular}{|c|c|c|c|c|c|}
\hline I & $\mid \backslash \neg(\mathrm{P} \supset \mathrm{Q}) \backslash$ & $\mathrm{P}$ & I & $\backslash \neg(\mathrm{P} \supset \mathrm{Q}) \backslash$ & $\mathrm{P}$ \\
\hline 2 & $\mid \neg P^{\prime}$ & $\mathrm{A}(c, \neg \mathrm{E})$ & 2 & $\perp^{\prime} \mathrm{Q}^{\prime}$ & $\mathrm{A}(c, \neg \mathrm{I})$ \\
\hline 3 & $\backslash P \backslash$ & $\mathrm{A}(g, \supset \mathrm{I})$ & 3 & $\mid \backslash P \backslash$ & $\mathrm{A}(g, \supset \mathrm{I})$ \\
\hline 4 & $\mid \neg Q \backslash$ & $\mathrm{A}(c, \neg \mathrm{E})$ & 4 & $/ Q^{\prime}$ & $2 \mathrm{R}$ \\
\hline 5 & $\backslash P \backslash$ & $3 \mathrm{R}$ & 5 & $/ \mathrm{P} \supset \mathrm{Q}^{\prime}$ & 3-4 つI \\
\hline 6 & $/ \neg P^{\prime}$ & $2 \mathrm{R}$ & 6 & $\mid \backslash \neg(\mathrm{P} \supset \mathrm{Q}) \backslash$ & \\
\hline 7 & /Q/ & $4^{-6} \neg \mathrm{E}$ & 7 & $\backslash \neg \mathrm{Q} \backslash$ & $2-6 \neg I$ \\
\hline 8 & $/ \mathrm{P} \supset \mathrm{Q} /$ & 3-7 つI & & & \\
\hline & $\backslash \neg(\mathrm{P} \supset \mathrm{Q}) \backslash$ & I $\mathrm{R}$ & & & \\
\hline & $\backslash \mathrm{P} \backslash$ & $2-9 \neg E$ & & & \\
\hline
\end{tabular}

that $\Gamma^{\prime} \vdash_{\mathrm{NMx}}^{*} \backslash \mathrm{P} \backslash$ and $\Gamma^{\prime} \vdash_{\mathrm{NMx}}^{*} \backslash \neg \mathrm{Q} \backslash$; so by consistency, $\Gamma^{\prime} \nvdash_{\mathrm{NMx}}^{*} / \mathrm{Q} /$; so by assumption, $h(\backslash P \backslash)=1$ and $h(/ Q /)=0$; so by $\mathrm{HM}(\supset)$, $\mathrm{h}(/ \mathrm{P} \supset \mathrm{Q} /)=0$. This is impossible; reject the assumption: if $h(/ A /)=1$ then $\Gamma^{\prime} \vdash_{\text {NMx }}^{*} / A /$.

(ii) Suppose $\Gamma^{\prime} \vdash_{\mathrm{NMx}}^{*} / \mathrm{A} /$ but $\mathrm{h}(/ \mathrm{A} /)=0$; then $\Gamma^{\prime} \vdash_{\mathrm{NMx}}^{*} / \mathrm{P} \supset \mathrm{Q} /$ but $h(/ \mathrm{P} \supset \mathrm{Q} /)=0$. From the latter, by $\operatorname{HM}(\supset), h(\backslash \mathrm{P} \backslash)=1$ and $\mathrm{h}(/ \mathrm{Q} /)=0$; so by assumption, $\Gamma^{\prime} \vdash_{\mathrm{NMx}}^{*} \backslash \mathrm{P} \backslash$ and $\Gamma^{\prime} \nvdash_{\mathrm{NMx}}^{*} / \mathrm{Q} /$; but since $\Gamma^{\prime} \vdash_{\mathrm{NMx}}^{*} / \mathrm{P} \supset \mathrm{Q} /$ and $\Gamma^{\prime} \vdash_{\mathrm{NMx}}^{*} \backslash \mathrm{P} \backslash$, by $(\supset \mathrm{E}), \Gamma^{\prime} \vdash_{\mathrm{NMx}}^{*} / \mathrm{Q} /$. 
This is impossible; reject the assumption: if $\Gamma^{\prime} \vdash_{\mathrm{NMx}}^{*} / \mathrm{A} /$, then $h(/ A /)=1$. So $h(/ A /)=1$ iff $\Gamma^{\prime} \vdash_{\mathrm{NMx}}^{*} / A /$.

For any $A, h(/ A /)=1$ iff $\Gamma^{\prime} \vdash_{N M x}^{*} / A /$.

L6.8 If $\Gamma$ is consistent, then $v$ constructed as above is an $M x$ interpretation.

For this, we need to show that the relevant constraints are met. Suppose $\Gamma$ is consistent; by L6.4, $\Gamma^{\prime}$ is maximal; by L6.6, $\Gamma^{\prime}$ is consistent.

(exc) For systems $M C L$ and $M_{K}$ with $v(p) \neq\{1,0\}$, (D) is in NKx. Suppose $v(p)=\{1,0\}$; then $1 \in v(p)$ and $0 \in v(p)$; so by construction, $\Gamma^{\prime} \vdash_{\mathrm{NMx}}^{*} \mathrm{p}$ and $\Gamma^{\prime} \nvdash_{\mathrm{NMx}}^{*} \overline{\mathrm{p}}$; from the latter, by maximality, $\Gamma^{\prime} \vdash_{\mathrm{NMx}}^{*} \neg \mathrm{p}$; so by (D), $\Gamma^{\prime} \vdash_{\mathrm{NMx}}^{*} \neg \mathrm{p}$; so $\Gamma^{\prime}$ is inconsistent. This is impossible; reject the assumption: $v(p) \neq\{1,0\}$.

(exh) For systems $M C L$ and $M_{L P}$ with $v(p) \neq \phi$, (U) is in NKx. Suppose $v(p)=\phi$; then $1 \notin v(p)$ and $0 \notin v(p)$; so by construction, $\Gamma^{\prime} \nvdash_{N M x}^{*}$ $\mathrm{p}$ and $\Gamma^{\prime} \vdash_{\mathrm{NMx}}^{*} \bar{p}$; from the former, by maximality, $\Gamma^{\prime} \vdash_{\mathrm{NMx}}^{*} \bar{\neg}$; so by $(\mathrm{U}), \Gamma^{\prime} \vdash_{\mathrm{NMx}}^{*} \neg p$; so $\Gamma^{\prime}$ is inconsistent. This is impossible; reject the assumption: $v(p) \neq \phi$.

L6.9 If $\Gamma$ is consistent, then $h(\Gamma)=1$.

Suppose $\Gamma$ is consistent and / $A / \in \Gamma$; then by construction, $/ A / \in \Gamma^{\prime}$; so $\Gamma^{\prime} \vdash_{\mathrm{NMx}}^{*} / \mathrm{A} /$; so since $\Gamma$ is consistent, by L6.7, $\mathrm{h}(/ \mathrm{A} /)=1$. And similarly for any $/ A / \in \Gamma$. So $h(\Gamma)=1$.

Main result: Suppose $\Gamma \models_{M x} A$ but $\Gamma \nvdash_{N M x} A$. Then $\Gamma \models_{M x}^{*} A$ but $\Gamma \nvdash_{N M x}^{*} A$. By (DN), if $\Gamma \vdash_{\mathrm{NMx}}^{*} \neg \neg A$, then $\Gamma \vdash_{\mathrm{NMx}}^{*} A$; so $\Gamma \nvdash_{\mathrm{NMx}}^{*} \neg \neg A$; so by L6.2, $\Gamma \cup$ $\{\overline{\neg A}\}$ is consistent; so by L6.8 and L6.9, there is an $M x$ interpretation $v$ with corresponding $h$ constructed as above such that $h(\Gamma \cup\{\overline{\neg A}\})=1$; so $h(\overline{\neg A})=1$; so by $\mathrm{HM}(\neg), h(A)=0$; so $h(\Gamma)=1$ and $h(A)=0$; so by VMx* $\Gamma^{*} \nvdash_{M x}^{*} A$. This is impossible; reject the assumption: if $\Gamma \vdash_{M x} A$, then $\Gamma \vdash_{N M x} A$.

\section{BASIC RELEVANT LOGIC: $v X$ (CH. 9)}

\section{I LANGUAGE / SEMANTIC NOTIONS}

This section is developed directly in terms introduced for "expanded" notions of validity in demonstration of soundness and completeness in section 6. Apart from that discussion, the notions should be roughly familiar from derivations in that section.

LuX The VOCABUlary consists of propositional parameters $p_{0}, p_{1} \ldots$ with the operators, $\neg, \wedge, \vee$, and $\rightarrow$. Each propositional parameter is a FORMULA; if $A$ and $B$ are formulas, so are $\neg A,(A \wedge B),(A \vee B)$, and $(A \rightarrow B)$. $A \supset B$ abbreviates $\neg A \vee B$, and $A \equiv B$ abbreviates $(A \supset B) \wedge(B \supset A)$. 
This time, from the start, for any formula $A$, we allow $A$ and $\bar{A}$, where as before $/ A$ / and $\backslash A \backslash(/ / A / /$ and $\backslash A \|$ ) represent one or the other (and similarly for $N$ and $\bar{N}$ immediately below).

IvX An interpretation is $\langle W, N, \bar{N}, h\rangle$ where $W$ is a set of worlds, and $\mathrm{N}, \overline{\mathrm{N}} \subseteq W$ are normal worlds for truth and non-falsity respectively; $h$ is a function such that for any $w \in W, h_{w}(/ p /)=1$ or $h_{w}(/ p /)=0$, and for any $w$ not in $/ \mathrm{N} / \mathrm{h}_{w}(/ \mathrm{A} \rightarrow \mathrm{B} /)=1$ or $\mathrm{h}_{w}(/ \mathrm{A} \rightarrow \mathrm{B} /)=0$. So $h$ makes assignments directly to experssions of the sort $/ A \rightarrow B /$ at worlds not in $/ N /$. Say $/ A /$ holds at $w$ if $h_{w}(/ A /)=1$ and otherwise fails. Interpretations may also be subject to the constraints,

$$
\begin{array}{rlrl}
\mathrm{K} & \mathrm{N} & =\overline{\mathrm{N}}=\mathrm{W} \\
4 & \mathrm{~N} & =\overline{\mathrm{N}}
\end{array}
$$

The $K$ systems are subject to constraint (K), the 4 systems to (4). Of course, (K) implies (4); so it is enough that interpretations for $v K_{4}$ and $v K_{*}$ are subject to $(K) ; v N_{4}$ is subject to (4), and $v N_{*}$ to neither. With restriction $K, h$ reduces to a simple assignment to parameters at worlds.

$\mathrm{H} v$ For expressions not assigned a value directly,

$(\neg) h_{w}(\neg A /)=1$ if $h_{w}(\backslash A \backslash)=0$, and 0 otherwise.

$(\wedge) h_{w}(/ A \wedge B /)=1$ if $h_{w}(/ A /)=1$ and $h_{w}(/ B /)=1$, and 0 otherwise.

(V) $h_{w}(/ A \vee B /)=1$ if $h_{w}(/ A /)=1$ or $h_{w}(/ B /)=1$, and 0 otherwise.

$(\rightarrow)_{4}$ For $w \in / N /, h_{w}(/ A \rightarrow B /)=1$ iff there is no $x \in W$ such that $h_{x}(A)=1$ and $h_{x}(/ B /)=0$.

$(\rightarrow)_{*}$ For $w \in / N /, h_{w}(/ A \rightarrow B /)=1$ iff there is no $x \in W$ such that $h_{x}(/ / A / /)=1$ and $h_{x}(/ / B / /)=0$.

The 4 -systems $v \mathrm{~N}_{4}$ and $v \mathrm{~K}_{4}$ take $\mathrm{Hv}(\rightarrow)_{4}$; the star systems $v \mathrm{~N}_{*}$ and $v \mathrm{~K}_{*}$ take $\mathrm{H} v(\rightarrow)_{*}$. Where $\Gamma$ does not include formulas with overlines, $h_{w}(\Gamma)=1$ iff $h_{w}(A)=1$ for each $A \in \Gamma$; then,

$\operatorname{VvX} \Gamma \models_{v X} A$ iff there is no $v X$ interpretation $\langle W, N, \bar{N}, h\rangle$ and $w \in N$ such that $h_{w}(\Gamma)=1$ and $h_{w}(A)=0$.

As in the previous section, the single account is meant to accommodate different presentations in Priest, and help exhibit their differences. In particular, as for the previous section, given constraint (4), an interpretation $\langle W, N, \bar{N}, h\rangle$ corresponds to a relational $\langle W, N, \rho\rangle$, where $h_{w}(A)=1$ iff $A$ bears relation $\rho$ (which, as in the previous section, may be set membership) to I at $w$, and $h_{w}(\bar{A})=1$ iff $A$ does not bear $\rho$ to 0 at $w$. And an interpretation $\langle W, N, \bar{N}, h\rangle$ 
corresponds to a star interpretation $\langle\mathrm{W}, \mathrm{N}, *, v\rangle$ where $h_{w}(A)=1$ iff $v_{w}(A)=1$ and $h_{w}(\bar{A})=1$ iff $v_{w^{*}}(A)=1 .^{6}$

\subsection{NATURAL DERIVATIONS: NuX}

Allow expressions with both integer subscripts and overlines. I- and E- rules for $\neg, \wedge, \vee, \supset$ and $\equiv$ are a natural combination of rules for $N K v$ and NFDE, with rules for $\supset$ and $\equiv$ now derived.

$\mathrm{R} \mid \begin{aligned} & / \mathrm{P} / \mathrm{s} \\ & / \mathrm{P} / \mathrm{s}\end{aligned}$

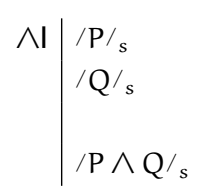<smiles>CCNCCOC</smiles>

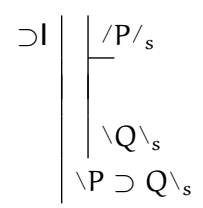<smiles>CC(C(=O)O)C(=O)O</smiles>

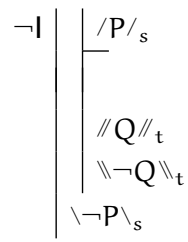

$\wedge \mathrm{E} \mid \begin{aligned} & / \mathrm{P} \wedge \mathrm{Q} / \mathrm{s} \\ & / \mathrm{P} / \mathrm{s}\end{aligned}$<smiles>[Mg][Mg][Mg]</smiles>

$\supset \mathrm{E} \mid \backslash \mathrm{P} \supset \mathrm{Q} \backslash_{\mathrm{s}}$ $/ \mathrm{P} / \mathrm{s}$ $\backslash \mathrm{Q} \backslash_{\mathrm{s}}$

$\equiv \mathrm{E} \mid \begin{aligned} & \backslash \mathrm{P} \equiv \mathrm{Q} \backslash_{\mathrm{s}} \\ & / \mathrm{P} / \mathrm{s} \\ & \backslash \mathrm{Q} \backslash_{\mathrm{s}}\end{aligned}$

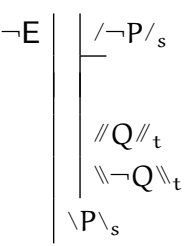

$\wedge \mathrm{E} \mid \begin{aligned} & / \mathrm{P} \wedge \mathrm{Q} / \mathrm{s} \\ & / \mathrm{Q} / \mathrm{s}\end{aligned}$



$\equiv \mathrm{E} \mid \backslash \mathrm{P} \equiv \mathrm{Q} \backslash_{s}$ $/ \mathrm{Q} / \mathrm{s}$ $\backslash \mathrm{P} \backslash_{S}$

The different derivation systems of this section add to these from,

\footnotetext{
${ }^{6}$ For the latter, given a star interpretation $\langle\mathrm{W}, \mathrm{N}, *, v\rangle$ consider an $v \mathrm{X}_{*}$ interpretation $\left\langle\mathrm{W}^{\prime}, \mathrm{N}^{\prime}, \overline{\mathrm{N}}^{\prime}, \mathrm{h}\right\rangle$ with a $w^{\prime} \in \mathrm{W}^{\prime}$ corresponding to each $w \in W$. And for an $v \mathrm{X}_{*}$ interpretation $\left\langle\mathrm{W}^{\prime}, \mathrm{N}^{\prime}, \overline{\mathrm{N}}^{\prime}, \mathrm{h}\right\rangle$ consider a star interpretation $\langle\mathrm{W}, \mathrm{N}, *, v\rangle$ with a $w$ and $w^{*} \in \mathrm{W}$ corresponding to each $w^{\prime} \in W^{\prime}$. Then set $x^{\prime} \in N^{\prime}$ iff $x \in N ; x^{\prime} \in \bar{N}^{\prime}$ iff $x^{*} \in N ; h_{x^{\prime}}(p)=1$ iff $v_{x}(p)=1 ; h_{x^{\prime}}(\bar{p})=1$ iff $v_{x^{*}}(p)=1$; for $x^{\prime} \notin N^{\prime}, h_{x^{\prime}}(P \rightarrow Q)=1$ iff $v_{x}(P \rightarrow Q)=1$; and for $x^{\prime} \notin \bar{N}^{\prime}, h_{x^{\prime}}(\overline{P \rightarrow Q})=1$ iff $v_{x^{*}}(\mathrm{P} \rightarrow \mathrm{Q})=1$. Then the result follows by a simple induction (for a related demonstration, see the proof of L7.0 below).
} 


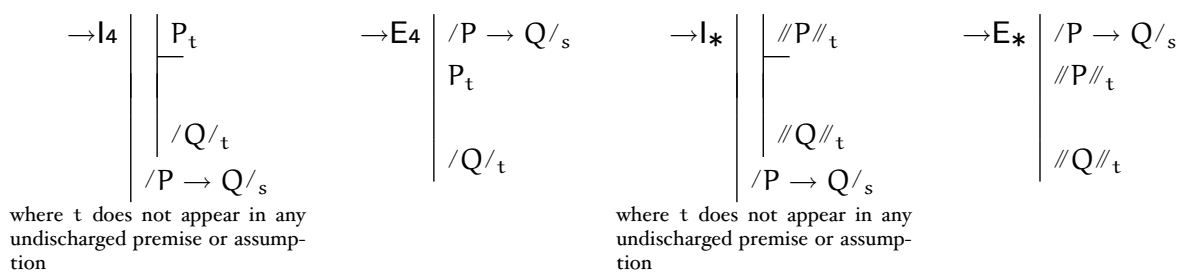

For the star-rules, //P//t and //Q//t may be either $P_{t}$ and $Q_{t}$, or $\bar{P}_{t}$ and $\bar{Q}_{t}$. Consider a constraint (n) which requires that $s=0$ for application of $\rightarrow I$ and $\rightarrow \mathrm{E}$, and a stronger constraint (s) which requires that $/ \mathrm{P} \rightarrow \mathrm{Q} / \mathrm{s}$ for these rules is of the sort $(\mathrm{P} \rightarrow \mathrm{Q})_{0}$ with subscript 0 and without overline. Then,

$\mathrm{NuK}_{4}$ adds $\rightarrow \mathrm{I}_{4}$ and $\rightarrow \mathrm{E}_{4}$

$\mathrm{N} \cup N_{4}$ adds $\rightarrow \mathrm{I}_{4}$ and $\rightarrow \mathrm{E}_{4}$ with constraint (n)

${\mathrm{N} v K_{*}}_{*}$ adds $\rightarrow \mathrm{I}_{*}$ and $\rightarrow \mathrm{E}_{*}$

$\mathrm{N} \cup \mathrm{N}_{*}$ adds $\rightarrow \mathrm{I} *$ and $\rightarrow \mathrm{E} *$ with constraint $(\mathrm{s})$

In these systems, every subscript is 0 , appears in a premise, or appears in the $t$-place of an accessible assumption for $\rightarrow I$. Where the members of $\Gamma$ and $A$ are without overlines or subscripts, let $\Gamma_{0}$ be the members of $\Gamma$, each with subscript 0. Then,

$\mathrm{NuX} \Gamma \vdash_{\mathrm{NuX}} A$ iff there is an $\mathrm{NuX}$ derivation of $A_{0}$ from $\Gamma_{0}$.

Derived rules are as one would expect. Two-way derived rules carry over from $C L$ with overlines and subscripts constant throughout. Thus, e.g.,

Impl $/ \mathrm{P} \supset \mathrm{Q} / \mathrm{s} \Delta \triangleright / \neg \mathrm{P} \vee \mathrm{Q} / \mathrm{s}$

$\neg \neg \supset \mathrm{Q} / \mathrm{s} \quad \triangleleft \triangleright / \mathrm{P} \vee \mathrm{Q} / \mathrm{s}$

MT, NB and DS appear in the forms,

$\mathrm{MT} \mid \begin{aligned} & / \mathrm{P} \supset \mathrm{Q} / \mathrm{s} \\ & \neg \neg \mathrm{Q} \backslash_{\mathrm{s}} \\ & / \neg \mathrm{P} / \mathrm{s}\end{aligned}$

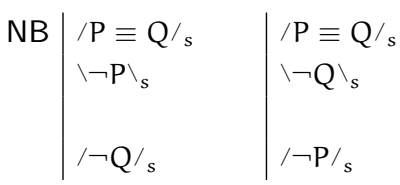

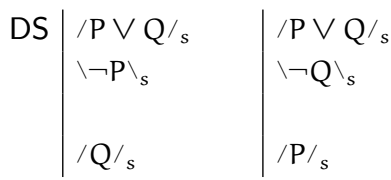

As examples, here are a few cases where the logics do not all have the same results.

\begin{tabular}{|c|c|c|}
\hline \multicolumn{3}{|c|}{$\mathrm{P} \rightarrow \mathrm{Q} \vdash_{\mathrm{NuX}_{*}} \neg \mathrm{Q} \rightarrow \neg \mathrm{P}$} \\
\hline & $(P \rightarrow Q)_{0}$ & $\mathrm{P}$ \\
\hline 2 & $\neg \mathrm{Q}_{1}$ & $\mathrm{~A}\left(g, \rightarrow \mathrm{I}_{*}\right)$ \\
\hline & $\overline{\mathrm{P}}_{1}$ & $\mathrm{~A}(c, \neg \mathrm{I})$ \\
\hline & $\overline{\mathrm{Q}}_{1}$ & $\mathrm{I}, 3 \rightarrow \mathrm{E}_{*}$ \\
\hline & $\neg Q_{1}$ & \\
\hline & $\begin{array}{l}\neg \mathrm{P}_{1} \\
(\neg \mathrm{Q} \rightarrow \neg \mathrm{P})_{0}\end{array}$ & $\begin{array}{l}3-5 \neg \mathrm{I} \\
2-6 \rightarrow \mathrm{I}_{*}\end{array}$ \\
\hline
\end{tabular}


This derivation satisfies constraints (n) and (s), but does not go through in the 4-systems insofar as there is no "purchase" for application of $\rightarrow \mathrm{E}_{4}$ with (I) and only $\bar{P}_{1}$, rather than $\mathrm{P}_{1}$, at (3).

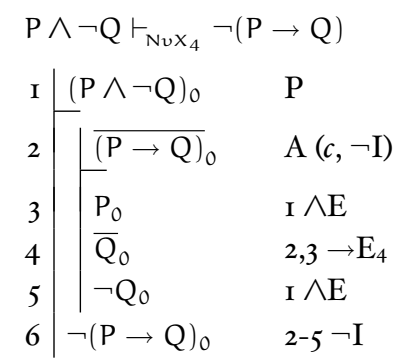

This derivation satisfies constraint (n), though not (s). It is blocked in either star system insofar as the contradiction does not arise; by $\rightarrow \mathrm{E}_{*}$, we might get $\mathrm{Q}_{0}$ at (4), but this does not contradict $\neg \mathrm{Q}_{0}$ for $\neg \mathrm{I}$.

\begin{tabular}{|c|c|c|}
\hline \multicolumn{3}{|c|}{$\vdash_{\mathrm{NuK}_{x}}[(\mathrm{P} \rightarrow \mathrm{Q}) \wedge(\mathrm{Q} \rightarrow \mathrm{R})] \rightarrow(\mathrm{P} \rightarrow \mathrm{R})$} \\
\hline & {$[(\mathrm{P} \rightarrow \mathrm{Q}) \wedge(\mathrm{Q} \rightarrow \mathrm{R})]_{1}$} & $\mathrm{~A}\left(g, \rightarrow \mathrm{I}_{x}\right)$ \\
\hline & $\mathrm{P}_{2}$ & $\mathrm{~A}\left(g, \rightarrow \mathrm{I}_{x}\right)$ \\
\hline & $(\mathrm{P} \rightarrow \mathrm{Q})_{1}$ & I $\wedge \mathrm{E}$ \\
\hline & $\mathrm{Q}_{2}$ & $2,3 \rightarrow \mathrm{E}_{x}$ \\
\hline & $(\mathrm{Q} \rightarrow \mathrm{R})_{1}$ & I $\wedge \mathrm{E}$ \\
\hline & $R_{2}$ & $4,5 \rightarrow \mathrm{E}_{x}$ \\
\hline & $(P \rightarrow R)_{1}$ & $2-6 \rightarrow I_{x}$ \\
\hline & $([(P \rightarrow Q) \wedge(Q \rightarrow R)] \rightarrow(P \rightarrow R))_{0}$ & $\mathrm{I}-7 \rightarrow \mathrm{I} x$ \\
\hline
\end{tabular}

This derivation works with either the star- or 4-rules. But it fails constraints (n) and (s) insofar as $s=1$ for lines (4), (6) and (7).

\subsection{SOUNDNESS AND COMPLETENESS}

Preliminaries: Begin with generalized notions of validity. Given any model $\langle W, N, \bar{N}, h\rangle$, let $m$ be a map from subscripts into $W$ such that $m(0)$ is some member of $N$. Then say $\langle W, N, \bar{N}, h\rangle_{m}$ is $\langle W, N, \bar{N}, h\rangle$ with map $m$. Then, where $\Gamma$ is a set of expressions of our language for derivations, $h_{m}(\Gamma)=1$ iff for each $/ A_{s} / \in \Gamma, h_{m(s)}(/ A /)=1$. Now expand notions of validity for subscripts and overlines as follows,

$\mathrm{VvX}^{*} \Gamma \models_{v \mathrm{x}}^{*} / \mathrm{A} / \mathrm{s}$ iff there is no $v \mathrm{X}$ interpretation $\langle\mathrm{W}, \mathrm{N}, \overline{\mathrm{N}}, \mathrm{h}\rangle_{\mathrm{m}}$ such that $h_{m}(\Gamma)=1$ but $h_{m(s)}(/ A /)=0$.

$\mathrm{NuX}^{*} \Gamma \vdash_{\mathrm{NuX}}^{*} / \mathrm{A} / \mathrm{s}$ iff there is an $\mathrm{N} v \mathrm{X}$ derivation of $/ \mathrm{A} / \mathrm{s}$ from the members of $\Gamma$.

These notions reduce to the standard ones when all the members of $\Gamma$ and $A$ are without overlines and have subscript 0 . As usual, for the following, cases omitted are like ones worked, and so left to the reader. 
THEOREM 7.I NuX is sound: If $\Gamma \vdash_{\mathrm{NuX}}$ A then $\Gamma \models_{v \mathrm{X}} \mathrm{A}$.

For the $(\rightarrow)_{*}$ case, it will be useful to have a further preliminary.

L7.0 For an interpretation $\langle W, N, \bar{N}, h\rangle$, consider $\left\langle W^{\prime}, N^{\prime}, \bar{N}^{\prime}, h^{\prime}\right\rangle$ such that corresponding to each $w \in W$ there are $w^{\prime}, w^{*} \in W^{\prime}$ where, (i) $w^{\prime} \in$ $/ \mathrm{N}^{\prime} /$ iff $w \in / \mathrm{N} /$, and $w^{*} \in / \mathrm{N}^{\prime} /$ iff $w \in \backslash \mathrm{N} \backslash$; (ii) $\mathrm{h}_{w^{\prime}}^{\prime}(/ \mathrm{p} /)=1$ iff $h_{w}(/ p /)=1$, and $h_{w^{*}}^{\prime}(/ p /)=1$ iff $h_{w}(\backslash p \backslash)=1$; (iii) for $w^{\prime} \notin / N^{\prime} /$, $h_{w^{\prime}}^{\prime}(/ \mathrm{P} \rightarrow \mathrm{Q} /)=1$ iff $h_{w}(/ \mathrm{P} \rightarrow \mathrm{Q} /)=1$, and for $w^{*} \notin / \mathrm{N}^{\prime} /, h_{w^{*}}^{\prime}(/ \mathrm{P} \rightarrow$ $\left.\mathrm{Q}^{\prime}\right)=1$ iff $\mathrm{h}_{w}(\backslash \mathrm{P} \rightarrow \mathrm{Q} \backslash)=1$. Then,

For the star systems and interpretations as above, for any / $\mathrm{A} /$, we have (i) $h_{w^{\prime}}^{\prime}(/ A /)=1$ iff $h_{w}(/ A /)=1$ and (ii) $h_{w^{*}}^{\prime}(/ A /)=1$ iff $h_{w}(\backslash A \backslash)=1$.

Basis: $/ A /$ is an atomic $/ p /$. (i) By construction, $h_{w^{\prime}}^{\prime}(/ p /)=1$ iff $h_{w}(/ p /)=1 ;$ so $h_{w^{\prime}}^{\prime}(/ A /)=1$ iff $h_{w}(/ A /)=1$. Similarly, (ii) by construction, $h_{w^{*}}^{\prime}(/ p /)=1$ iff $h_{w}(\backslash p \backslash)=1$; so $h_{w^{*}}^{\prime}(/ A /)=1$ iff $h_{w}(\backslash A \backslash)=1$.

Assp: For any $i, 0 \leqslant i<k$, if $/ A /$ has $i$ operators, (i) $h_{w^{\prime}}^{\prime}(/ A /)=1$ iff $h_{w}(/ A /)=1$ and (ii) $h_{w^{*}}^{\prime}(/ A /)=1$ iff $h_{w}(\backslash A \backslash)=1$.

Show: If $/ A /$ has $k$ operators, then (i) $h_{w^{\prime}}^{\prime}(/ A /)=1$ iff $h_{w}(/ A /)=1$ and (ii) $h_{w^{*}}^{\prime}(/ A /)=1$ iff $h_{w}(\backslash A \backslash)=1$.

If $/ A /$ has $k$ operators, then it is of the form, $/ \neg P /, / P \wedge Q /$, $/ \mathrm{P} \vee \mathrm{Q} /$, or $/ \mathrm{P} \rightarrow \mathrm{Q} /$, where $\mathrm{P}$ and $\mathrm{Q}$ have $<\mathrm{k}$ operators.

$(\neg) / A /$ is $/ \neg \mathrm{P} /$. (i) $h_{w^{\prime}}^{\prime}(/ \mathrm{A} /)=1$ iff $h_{w^{\prime}}^{\prime}(/ \neg \mathrm{P} /)=1$; by $\mathrm{Hv}(\neg)$, iff $h_{w^{\prime}}^{\prime}(\backslash P \backslash)=0$; by assumption iff $h_{w}(\backslash P \backslash)=0$; by $\mathrm{Hv}(\neg)$, iff $h_{w}(/ \neg P /)=1$; iff $h_{w}(/ A /)=1$. (ii) (i) $h_{w^{*}}^{\prime}(/ A /)=1$ iff $h_{w^{*}}^{\prime}(/ \neg \mathrm{P} /)=1$; by $\mathrm{Hv}(\neg)$, iff $h_{w^{*}}^{\prime}(\backslash \mathrm{P} \backslash)=0$; by assumption iff $h_{w}(/ P /)=0$; by $H v(\neg)$, iff $h_{w}(\backslash \neg P \backslash)=1$; iff $h_{w}(\backslash A \backslash)=1$.

( $) / A /$ is $/ P \wedge Q /$. (i) $h_{w^{\prime}}^{\prime}(/ A /)=1$ iff $h_{w^{\prime}}^{\prime}(/ P \wedge Q /)=1$; by $\mathrm{H} v(\wedge)$, iff $h_{w^{\prime}}^{\prime}(/ \mathrm{P} /)=1$ and $h_{w^{\prime}}^{\prime}(/ \mathrm{Q} /)=1$; by assumption, iff $h_{w}(/ \mathrm{P} /)=1$ and $h_{w}(/ \mathrm{Q} /)=1$; by $\mathrm{H} v(\wedge)$, iff $h_{w}(/ \mathrm{P} \wedge \mathrm{Q} /)=1$; iff $h_{w}(/ A /)=1$. (ii) $h_{w^{*}}^{\prime}(/ A /)=1$ iff $h_{w^{*}}^{\prime}(/ P \wedge Q /)=1$; by $\mathrm{H} v(\wedge)$, iff $h_{w^{*}}^{\prime}(/ \mathrm{P} /)=1$ and $h_{w^{*}}^{\prime}(/ \mathrm{Q} /)=1$; by assumption, iff $h_{w}(\backslash P \backslash)=1$ and $h_{w}(\backslash Q \backslash)=1$; by $H v(\wedge)$, iff $h_{w}(\backslash P \wedge Q \backslash)=1$; iff $h_{w}(\backslash A \backslash)=1$.

$(\mathrm{V})$

$(\rightarrow) A$ is $/ \mathrm{P} \rightarrow \mathrm{Q} /$. (i) Suppose $w^{\prime} \notin / \mathrm{N}^{\prime} /$; then by construction, $h_{w^{\prime}}^{\prime}(/ \mathrm{P} \rightarrow \mathrm{Q} /)=1$ iff $h_{w}(/ \mathrm{P} \rightarrow \mathrm{Q} /)=1 ;$ so $h_{w^{\prime}}^{\prime}(/ \mathrm{A} /)=1$ iff $h_{w}(/ A /)=1$. So suppose $w^{\prime} \in / N^{\prime} /$; then by construction, $w \in / \mathrm{N} / . \mathrm{h}_{w^{\prime}}^{\prime}(/ \mathrm{A} /)=0$ iff $\mathrm{h}_{w^{\prime}}^{\prime}(/ \mathrm{A} \rightarrow \mathrm{B} /)=0$; since $w^{\prime} \in / \mathrm{N}^{\prime} /$, by $\mathrm{H} v(\rightarrow)_{*}$ iff either there is an $x^{\prime} \in W^{\prime}$ such that $h_{x^{\prime}}^{\prime}(/ / \mathrm{P} / /)=1$ and $h_{x^{\prime}}^{\prime}(/ / \mathrm{Q} / /)=0$, or there is a $y^{*} \in W^{\prime}$ such that $h_{y^{*}}^{\prime}(/ / \mathrm{P} / /)=1$ and $h_{y^{*}}^{\prime}(/ / \mathrm{Q} / /)=0$; by assumption, iff either $h_{x}(/ / \mathrm{P} / /)=1$ and 
$h_{x}(/ / Q / /)=0$, or $h_{y}(\|P\|)=1$ and $h_{y}(\backslash Q \|)=0$; given either of these, since $w \in / \mathrm{N} /$, by $\mathrm{Hv}(\rightarrow)_{*}$, iff $h_{w}(/ \mathrm{P} \rightarrow \mathrm{Q} /)=0$; iff $h_{w}(/ A /)=0$.

(ii) Suppose $w^{*} \notin / N^{\prime} /$; then by construction, $h_{w^{*}}^{\prime}(/ P \rightarrow Q /)=1$ iff $h_{w}(\backslash P \rightarrow Q \backslash)=1$; so $h_{w^{*}}^{\prime}(/ A /)=1$ iff $h_{w}(\backslash A \backslash)=1$. So suppose $w^{*} \in / N^{\prime} /$; then $w \in \backslash N \backslash . h_{w^{*}}^{\prime}(/ A /)=0$ iff $h_{w^{*}}^{\prime}(/ A \rightarrow$ $\mathrm{B} /)=0$; since $w^{*} \in / \mathrm{N}^{\prime} /$, by $\mathrm{Hv}(\rightarrow)_{*}$ iff either there is an $x^{\prime} \in$ $W^{\prime}$ such that $h_{x^{\prime}}^{\prime}(/ / \mathrm{P} / /)=1$ and $h_{x^{\prime}}^{\prime}(/ / \mathrm{Q} / /)=0$, or there is a $y^{*} \in$ $W^{\prime}$ such that $h_{y^{*}}^{\prime}(/ / \mathrm{P} / /)=1$ and $h_{y^{*}}^{\prime}(/ / \mathrm{Q} / /)=0$; by assumption, iff either $h_{x}(/ / P / /)=1$ and $h_{x}(/ / Q / /)=0$, or $h_{y}(\|P\|)=1$ and $h_{y}(\backslash Q \|)=0$; given either of these, since $w \in \backslash N \backslash$, by $\mathrm{Hv}(\rightarrow)_{*}$, iff $h_{w}(\backslash P \rightarrow Q \backslash)=0$; iff $h_{w}(\backslash A \backslash)=0$.

For any $A$, (i) $h_{w^{\prime}}^{\prime}(/ A /)=1$ iff $h_{w}(/ A /)=1$ and (ii) $h_{w^{*}}^{\prime}(/ A /)=1$ iff $h_{w}(\backslash A \backslash)=1$.

L7.I If $\Gamma \subseteq \Gamma^{\prime}$ and $\Gamma \models_{v x}^{*} / \mathrm{P} / \mathrm{s}$ then $\Gamma^{\prime} \models_{v x}^{*} / \mathrm{P} / \mathrm{s}$.

Suppose $\Gamma \subseteq \Gamma^{\prime}$ and $\Gamma \models_{v x}^{*} / \mathrm{P} / s$, but $\Gamma^{\prime} \forall_{v x}^{*} / \mathrm{P} / s$. From the latter, by VvX*, there is some $v X$ interpretation $\langle W, N, \bar{N}, h\rangle_{m}$ such that $h_{m}\left(\Gamma^{\prime}\right)=$ 1 but $h_{m(s)}(/ \mathrm{P} /)=0$. But since $h_{m}\left(\Gamma^{\prime}\right)=1$ and $\Gamma \subseteq \Gamma^{\prime}, h_{m}(\Gamma)=1$; so $h_{m}(\Gamma)=1$ but $h_{m(s)}(/ \mathrm{P} /)=0$; so by $\mathrm{VvX}^{*}, \Gamma \not \nvdash_{v x}^{*} / \mathrm{P} / \mathrm{s}$. This is impossible; reject the assumption: if $\Gamma \subseteq \Gamma^{\prime}$ and $\Gamma \models_{v x}^{*} / \mathrm{P} / s$, then $\Gamma^{\prime} \models_{v x}^{*} / \mathrm{P} / \mathrm{s}$.

Main result: For each line in a derivation let $\mathcal{P}_{i}$ be the expression on line $i$ and $\Gamma_{i}$ be the set of all premises and assumptions whose scope includes line $i$. We set out to show "generalized" soundness: if $\Gamma \vdash_{\text {Nox }}^{*} \mathcal{P}$ then $\Gamma \models_{v x}^{*} \mathcal{P}$. As above, this reduces to the standard result when $\mathcal{P}$ and all the members of $\Gamma$ are without overlines and have subscript 0 . Suppose $\Gamma \vdash_{\mathrm{Nux}}^{*} \mathcal{P}$. Then there is a derivation of $\mathcal{P}$ from premises in $\Gamma$ where $\mathcal{P}$ appears under the scope of the premises alone. By induction on line number of this derivation, we show that for each line $i$ of this derivation, $\Gamma_{i} \models_{v x}^{*} \mathcal{P}_{i}$. The case when $\mathcal{P}_{i}=\mathcal{P}$ is the desired result.

Basis: $\mathcal{P}_{1}$ is a premise or an assumption $/ \mathrm{A} / \mathrm{s}$. Then $\Gamma_{1}=\{/ \mathrm{A} / \mathrm{s}\}$; it follows that for any $\langle W, N, \bar{N}, h\rangle_{m}, h_{m}\left(\Gamma_{1}\right)=1$ iff $h_{m(s)}(/ A /)=1$; so there is no $\langle W, N, \bar{N}, h\rangle_{m}$ such that $h_{m}\left(\Gamma_{1}\right)=1$ but $h_{m(s)}(/ A /)=0$. So by $\mathrm{VvX}^{*}, \Gamma_{1} \models_{v \mathrm{x}}^{*} / \mathrm{A} / \mathrm{s}$, where this is just to say, $\Gamma_{1} \models_{v x}^{*} \mathcal{P}_{1}$.

Assp: For any $i, 1 \leqslant i<k, \Gamma_{i} \models_{v x}^{*} \mathcal{P}_{i}$.

Show: $\Gamma_{\mathrm{k}} \models_{\mathrm{vx}}^{*} \mathcal{P}_{\mathrm{k}}$.

$\mathcal{P}_{k}$ is either a premise, an assumption, or arises from previous lines by $\mathrm{R}, \wedge \mathrm{I}, \wedge \mathrm{E}, \vee \mathrm{I}, \vee \mathrm{E}, \neg \mathrm{I}, \neg \mathrm{E}$ or, depending on the system, $\rightarrow \mathrm{I}_{4}, \rightarrow \mathrm{E}_{4}$, $\rightarrow I_{*}$, or $\rightarrow E_{*}$. If $\mathcal{P}_{k}$ is a premise or an assumption, then as in the basis, $\Gamma_{k} \models_{v x}^{*} \mathcal{P}_{k}$. So suppose $\mathcal{P}_{k}$ arises by one of the rules. 
$(\neg \mathrm{I})$ If $\mathcal{P}_{k}$ arises by $\neg \mathrm{I}$, then the picture is like this,

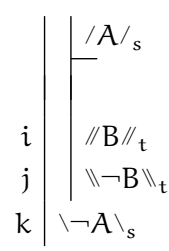

where $i, j<k$ and $\mathcal{P}_{k}$ is $\backslash \neg A \backslash_{s}$. By assumption, $\Gamma_{i} \models_{v x}^{*} / / B / / t$ and $\Gamma_{j} \models_{v x}^{*}\|\neg B\|_{t}$; but by the nature of access, $\Gamma_{i} \subseteq \Gamma_{k} \cup\{/ A / s\}$ and $\Gamma_{j} \subseteq$ $\Gamma_{\mathrm{k}} \cup\{/ \mathrm{A} / \mathrm{s}\}$; so by L7.I, $\Gamma_{\mathrm{k}} \cup\{/ \mathrm{A} / \mathrm{s}\} \models_{v \mathrm{x}}^{*} / / \mathrm{B} / / \mathrm{t}$ and $\Gamma_{\mathrm{k}} \cup\{/ \mathrm{A} / \mathrm{s}\} \models_{v \mathrm{x}}^{*}$ $\|\neg B\|_{t}$. Suppose $\Gamma_{k} \forall_{v x}^{*} \backslash \neg A \backslash_{s}$; then by VvX*, there is an $v X$ interpretation $\langle W, N, \bar{N}, h\rangle_{m}$ such that $h_{m}\left(\Gamma_{k}\right)=1$ but $h_{m(s)}(\backslash \neg A \backslash)=0$; so by $\mathrm{Hv}(\neg), h_{m(s)}(/ A /)=1$; so $h_{m}\left(\Gamma_{k}\right)=1$ and $h_{m(s)}(/ A /)=1$; so $h_{m}\left(\Gamma_{k} \cup\{/ A / s\}\right)=1$; so by $V v X^{*}, h_{m(t)}(/ / B / /)=1$ and $h_{m(t)}(\| \neg B \backslash)=$ 1; from the latter, by $\mathrm{Hv}(\neg), h_{m(t)}(/ / B / /)=0$. This is impossible; reject the assumption: $\Gamma_{k} \models_{v x}^{*} \backslash \neg A \backslash_{s}$, which is to say, $\Gamma_{k} \models_{v x}^{*} \mathcal{P}_{k}$.

$(\neg \mathrm{E})$

$\left(\rightarrow \mathrm{I}_{4}\right)$ If $\mathcal{P}_{\mathrm{k}}$ arises by $\rightarrow \mathrm{I}_{4}$, then the picture is like this,

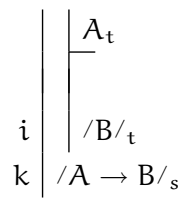

where $i<k$, $t$ does not appear in any member of $\Gamma_{k}$ (in any undischarged premise or assumption), and $\mathcal{P}_{k}$ is $/ A \rightarrow B / s$. For these systems, either by condition $\mathrm{K}, W=\mathrm{N}=\overline{\mathrm{N}}$ or by constraint (n), $\mathrm{s}=0$; in the first case, $m(s) \in N$ and $m(s) \in \bar{N}$; so $m(s) \in / N /$; in the other case, by the construction of $m, m(s) \in N$; so with $N=\bar{N}$ by condition (4), $\mathrm{m}(\mathrm{s}) \in \overline{\mathrm{N}}$; so $\mathrm{m}(\mathrm{s}) \in / \mathrm{N} /$; in either case, $\mathrm{m}(\mathrm{s}) \in / \mathrm{N} /$. By assumption, $\Gamma_{i} \models_{v x}^{*} / B / t$; but by the nature of access, $\Gamma_{i} \subseteq \Gamma_{k} \cup\left\{A_{t}\right\}$; so by L7.I, $\Gamma_{k} \cup\left\{A_{t}\right\} \models_{v x}^{*} / B / t$. Suppose $\Gamma_{k} \not \nvdash_{v x}^{*} / A \rightarrow B / s$; then by VvX*, there is an $v X$ interpretation $\langle W, N, \bar{N}, h\rangle_{m}$ such that $h_{m}\left(\Gamma_{k}\right)=1$ but $h_{m(s)}(/ A \rightarrow$ $\mathrm{B} /)=0$; so, since $\mathrm{m}(\mathrm{s}) \in / \mathrm{N} /$, by $\mathrm{H} v(\rightarrow)_{4}$, there is some $w \in W$ such that $h_{w}(A)=1$ and $h_{w}(/ B /)=0$. Now consider a map $m^{\prime}$ like 
$m$ except that $m^{\prime}(t)=w$, and consider $\langle W, N, \bar{N}, h\rangle_{m^{\prime}}$; since $t$ does not appear in $\Gamma_{k}$, it remains that $h_{m^{\prime}}\left(\Gamma_{k}\right)=1$; and since $m^{\prime}(t)=w$, $h_{m^{\prime}(t)}(A)=1$; so $h_{m^{\prime}}\left(\Gamma_{k} \cup\left\{A_{t}\right\}\right)=1$; so by $V v X^{*}, h_{m^{\prime}(t)}(/ B /)=1$. But $m^{\prime}(t)=w$; so $h_{w}(/ B /)=1$. This is impossible; reject the assumption: $\Gamma_{\mathrm{k}} \models_{v \mathrm{x}}^{*} / \mathrm{A} \rightarrow \mathrm{B} / \mathrm{s}$, which is to say, $\Gamma_{\mathrm{k}} \models_{v \mathrm{x}}^{*} \mathcal{P}_{\mathrm{k}}$.

$\left(\rightarrow \mathrm{E}_{4}\right)$ If $\mathcal{P}_{\mathrm{k}}$ arises by $\rightarrow \mathrm{E}_{4}$, then the picture is like this,

\begin{tabular}{l|l}
$i$ & $/ A \rightarrow B / s$ \\
$j$ & $A_{t}$ \\
$k$ & $/ B / t$
\end{tabular}

where $i, j<k$ and $\mathcal{P}_{k}$ is $/ \mathrm{B} / \mathrm{t}$. For these systems, either by condition $\mathrm{K}, \mathrm{W}=\mathrm{N}=\overline{\mathrm{N}}$ or by constraint (n), $\mathrm{s}=0$; in the first case, $\mathrm{m}(\mathrm{s}) \in \mathrm{N}$ and $\mathrm{m}(\mathrm{s}) \in \overline{\mathrm{N}}$; so $\mathrm{m}(\mathrm{s}) \in / \mathrm{N} /$; in the other case, by the construction of $\mathrm{m}, \mathrm{m}(\mathrm{s}) \in \mathrm{N}$; so with $\mathrm{N}=\overline{\mathrm{N}}$ by condition (4), $\mathrm{m}(\mathrm{s}) \in \overline{\mathrm{N}}$; so $\mathrm{m}(\mathrm{s}) \in$ $/ \mathrm{N} /$; in either case, $\mathrm{m}(\mathrm{s}) \in / \mathrm{N} /$. By assumption, $\Gamma_{\mathrm{i}} \models_{v \mathrm{v}}^{*} / \mathrm{A} \rightarrow \mathrm{B} / \mathrm{s}$ and $\Gamma_{j} \models_{v x}^{*} A_{t}$; but by the nature of access, $\Gamma_{i} \subseteq \Gamma_{k}$ and $\Gamma_{j} \subseteq \Gamma_{k}$; so by L7.I, $\Gamma_{k} \models_{v x}^{*} / A \rightarrow B / s$ and $\Gamma_{k} \models_{v x}^{*} A_{t}$. Suppose $\Gamma_{k} \not \models_{v x}^{*} / B / t$; then by VvX*, there is some $v X$ interpretation $\langle W, N, \bar{N}, h\rangle_{m}$ such that $h_{m}\left(\Gamma_{k}\right)=1$ but $h_{m(t)}(/ B /)=0$; since $h_{m}\left(\Gamma_{k}\right)=1$, by VvX*, $h_{m(s)}(/ A \rightarrow B /)=1$ and $h_{m(t)}(A)=1$; from the first of these, since $m(s) \in / N /$, by $\mathrm{Hv}(\rightarrow)_{4}$, there is no $w \in W$ such that $h_{w}(A)=1$ and $h_{w}(/ B /)=0$; so it is not the case that $h_{m(t)}(A)=1$ and $h_{m(t)}(/ B /)=0$. This is impossible; reject the assumption: $\Gamma_{k} \models_{v x}^{*} / B / t$, which is to say, $\Gamma_{k} \models_{v x}^{*} \mathcal{P}_{k}$.

$\left(\rightarrow I_{*}\right)$ If $\mathcal{P}_{k}$ arises by $\rightarrow I_{*}$, then the picture is like this,

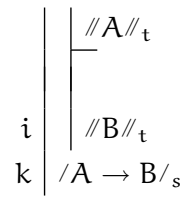

where $i<k$, $t$ does not appear in any member of $\Gamma_{k}$ (in any undischarged premise or assumption), and $\mathcal{P}_{k}$ is $/ A \rightarrow B / s$. For these systems, either by condition $\mathrm{K}, \mathrm{W}=\mathrm{N}=\overline{\mathrm{N}}$ or by constraint (s), $/ \mathrm{A} \rightarrow \mathrm{B} / \mathrm{s}$ is of the sort, $(A \rightarrow B)_{0}$; in the first case, $m(s) \in N$ and $m(s) \in \bar{N}$; so $m(s) \in / N /$; in the other case, $s=0$; so by the construction of $m, m(s) \in N$, which is to say $m(s) \in / N /$; so in either case, $m(s) \in / N /$. By assumption, $\Gamma_{\mathrm{i}} \models_{v \mathrm{x}}^{*} / / \mathrm{B} / / \mathrm{t}$; but by the nature of access, $\Gamma_{\mathrm{i}} \subseteq \Gamma_{\mathrm{k}} \cup\{/ / \mathrm{A} / / \mathrm{t}\}$; so by L7.I, $\Gamma_{\mathrm{k}} \cup\{/ / \mathrm{A} / / \mathrm{t}\} \models_{v x}^{*} / / \mathrm{B} / / \mathrm{t}$. Suppose $\Gamma_{\mathrm{k}} \not \nvdash_{v x}^{*} / \mathrm{A} \rightarrow \mathrm{B} / s$; then by VvX*, there is an $v X$ interpretation $\langle W, N, \bar{N}, h\rangle_{m}$ such that $h_{m}\left(\Gamma_{k}\right)=1$ but $h_{m(s)}(/ A \rightarrow B /)=0$; so by $\mathrm{Hv}(\rightarrow)_{*}$, there is some $x \in W$ such that $h_{x}(A)=1$ and $h_{x}(B)=0$, or $h_{x}(\bar{A})=I$ and $h_{x}(\bar{B})=0$. Without loss of generality, suppose $h_{x}(A)=1$ and $h_{x}(B)=0$; then by L7.0, there is an interpretation $\left\langle W^{\prime}, N^{\prime}, \bar{N}^{\prime}, h^{\prime}\right\rangle$ where $h_{w^{\prime}}^{\prime}(/ \mathrm{P} /)=1$ iff $h_{w}(/ \mathrm{P} /)=1$ 
and $h_{w^{*}}^{\prime}(/ P /)=1$ iff $h_{w}(\backslash P \backslash)=1$. So with $m(s)=w$ iff $m^{\prime}(s)=w^{\prime}$, it remains that $h_{m^{\prime}}^{\prime}\left(\Gamma_{k}\right)=1$; and we have that $x^{\prime}, x^{*} \in W^{\prime}$ are such that $h_{x^{\prime}}^{\prime}(A)=1$ and $h_{x^{\prime}}^{\prime}(B)=0$, and $h_{x^{*}}^{\prime}(\bar{A})=1$ and $h_{x^{*}}^{\prime}(\bar{B})=0$; one of these is a $y$ such that $h_{y}^{\prime}(/ / \mathrm{A} / /)=1$ and $h_{y}^{\prime}(/ / \mathrm{B} / /)=0$. Now consider a map $n$ like $m^{\prime}$ except that $n(t)=y$, and consider $\left\langle W^{\prime}, N^{\prime}, \bar{N}^{\prime}, h^{\prime}\right\rangle_{n}$; since $t$ does not appear in $\Gamma_{k}$, it remains that $h_{n}^{\prime}\left(\Gamma_{k}\right)=1$; and since $n(t)=y$, $h_{n(t)}^{\prime}(/ / A / /)=1 ;$ so $h_{n}^{\prime}\left(\Gamma_{k} \cup\{/ / A / / t\}\right)=1$; so by VvX*, $h_{n(t)}^{\prime}(/ / B / /)=1$. But $n(t)=y$; so $h_{y}^{\prime}(/ / B / /)=1$. This is impossible; reject the assumption: $\Gamma_{\mathrm{k}} \models_{v \mathrm{x}}^{*} / \mathrm{A} \rightarrow \mathrm{B} / \mathrm{s}$, which is to say, $\Gamma_{\mathrm{k}} \models_{v \mathrm{x}}^{*} \mathcal{P}_{\mathrm{k}}$.

$\left(\rightarrow \mathrm{E}_{*}\right)$ If $\mathcal{P}_{\mathrm{k}}$ arises by $\rightarrow \mathrm{E}_{*}$, then the picture is like this,

\begin{tabular}{l|l}
$i$ & $/ A \rightarrow B / s$ \\
$j$ & $/ / A / / t$ \\
$k$ & $/ / B / / t$
\end{tabular}

where $i, j<k$ and $\mathcal{P}_{k}$ is $/ / B / / t$. For these systems, either by condition $K, W=N=\bar{N}$ or by constraint (s), $/ A \rightarrow B / s$ is of the sort, $(A \rightarrow B)_{0}$; in the first case, $m(s) \in N$ and $m(s) \in \bar{N}$; so $m(s) \in / N /$; in the other case, $s=0$; so by the construction of $\mathrm{m}, \mathrm{m}(\mathrm{s}) \in \mathrm{N}$, which is to say $\mathrm{m}(\mathrm{s}) \in / \mathrm{N} /$; so in either case, $\mathrm{m}(\mathrm{s}) \in / \mathrm{N} /$. By assumption, $\Gamma_{\mathrm{i}} \models_{v x}^{*}$ $/ A \rightarrow B / s$ and $\Gamma_{j} \models_{v x}^{*} / / A / / t$; but by the nature of access, $\Gamma_{i} \subseteq \Gamma_{k}$ and $\Gamma_{\mathrm{j}} \subseteq \Gamma_{\mathrm{k}}$; so by $\mathrm{L} 7 . \mathrm{I}, \Gamma_{\mathrm{k}} \models_{v \mathrm{x}}^{*} / \mathrm{A} \rightarrow \mathrm{B} / \mathrm{s}$ and $\Gamma_{\mathrm{k}} \models_{v \mathrm{x}}^{*} / / \mathrm{A} / /{ }_{\mathrm{t}}$. Suppose $\Gamma_{\mathrm{k}} \forall_{v \mathrm{x}}^{*}$ $/ / \mathrm{B} /{ }_{t}$; then by $V v X^{*}$, there is some $v X$ interpretation $\langle W, N, \bar{N}, h\rangle_{m}$ such that $h_{m}\left(\Gamma_{k}\right)=1$ but $h_{m(t)}(/ / B / /)=0$; since $h_{m}\left(\Gamma_{k}\right)=1$, by VvX*, $h_{m(s)}(/ A \rightarrow B /)=1$ and $h_{m(t)}(/ / A / /)=1$; from the first of these, since $\mathrm{m}(\mathrm{s}) \in / \mathrm{N} /$, by $\mathrm{H} v(\rightarrow)_{*}$, there is no $w \in W$ such that $h_{w}(/ / A / /)=$ 1 and $h_{w}(/ / B / /)=0$; so it is not the case that $h_{m(t)}(/ / A / /)=1$ and $h_{m(t)}(/ / B / /)=0$. This is impossible; reject the assumption: $\Gamma_{k} \models_{v x}^{*}$ $/ / \mathrm{B} / / \mathrm{t}$, which is to say, $\Gamma_{\mathrm{k}} \models_{v x}^{*} \mathcal{P}_{k}$.

For any $i, \Gamma_{i} \models_{v x}^{*} \mathcal{P}_{i}$.

THEOREM 7.2 NuX is complete: if $\Gamma \models_{v X}$ A then $\Gamma \vdash_{\mathrm{NuX}} A$.

Suppose $\Gamma \models_{v x} A$; then $\Gamma_{0} \models_{v x}^{*} A_{0}$; we show that $\Gamma_{0} \vdash_{N v x}^{*} A_{0}$. As usual, this reduces to the standard notion. For the following, fix on some particular $v X$. Then definitions of consistency etc. are relative to it.

Con $\Gamma$ is Consistent iff there is no $A_{s}$ such that $\Gamma \vdash_{N u X}^{*} / A / s$ and $\Gamma \vdash_{\text {Nux }}^{*}$ $\backslash \neg A \backslash_{s}$.

L7.2 If $s$ is 0 or appears in $\Gamma$, and $\Gamma \nvdash_{N u X}^{*} \backslash \neg \mathrm{P} \backslash_{s}$, then $\Gamma \cup\{/ \mathrm{P} / s\}$ is consistent. 
Suppose $s$ is 0 or appears in $\Gamma$ and $\Gamma \nvdash_{N u x}^{*} \backslash \neg P \backslash_{s}$ but $\Gamma \cup\{/ \mathrm{P} / s\}$ is inconsistent. Then there is some $A_{t}$ such that $\Gamma \cup\{/ \mathrm{P} / s\} \vdash_{\mathrm{NuX}}^{*} / / \mathrm{A} / / \mathrm{t}$ and $\Gamma \cup\{/ \mathrm{P} / s\} \vdash_{\mathrm{Nux}}^{*}\|\neg A\|_{t}$. But then we can argue,

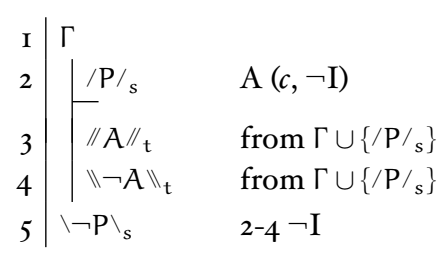

where the assumption is allowed insofar as $s$ is either 0 or appears in $\Gamma$; so $\Gamma \vdash_{\mathrm{NuX}}^{*} \backslash \neg \mathrm{P} \backslash_{s}$. But this is impossible; reject the assumption: if $s$ is 0 or appears in $\Gamma$ and $\Gamma \nvdash_{\mathrm{Nox}}^{*} \backslash \neg P \backslash_{s}$, then $\Gamma \cup\{/ \mathrm{P} / \mathrm{s}\}$ is consistent.

L7.3 There is an enumeration of all the subscripted formulas, $\mathcal{P}_{1} \mathcal{P}_{2} \ldots$

Proof by construction as usual.

Max $\Gamma$ is s-maximaL iff for any $A_{s}$ either $\Gamma \vdash_{\text {Nox }}^{*} / A /{ }_{s}$ or $\Gamma \vdash_{N o x}^{*} \backslash \neg A \backslash_{s}$.

SGT $\Gamma$ is a SCAPEGOAT set for $(\rightarrow)_{K_{4}}$ iff for every formula of the form $\neg(A \rightarrow$ B), if $\Gamma \vdash_{\mathrm{NuK}_{4}}^{*} / \neg(A \rightarrow B) / s$ then there is some $t$ such that $\Gamma \vdash_{\mathrm{NuK}_{4}}^{*} A_{t}$ and $\Gamma \vdash_{\mathrm{NuK}_{4}}^{*} / \neg \mathrm{B} / \mathrm{t}$.

$\Gamma$ is a SCAPEGOAT set for $(\rightarrow)_{N_{4}}$ iff for every formula of the form $\neg(A \rightarrow$ B), if $\Gamma \vdash_{\mathrm{NuK}_{4}}^{*} / \neg(A \rightarrow B) / 0$ then there is some $t$ such that $\Gamma \vdash_{\mathrm{NuK}_{4}}^{*} A_{t}$ and $\Gamma \vdash_{\mathrm{NuK}_{4}}^{*} / \neg \mathrm{B} / \mathrm{t}$.

$\Gamma$ is a SCAPEGOAT set for $(\rightarrow)_{K_{*}}$ iff for every formula of the form $\neg(A \rightarrow$ B), if $\Gamma \vdash_{\mathrm{NuK}_{*}}^{*} / \neg(A \rightarrow B) / s$ then there is some $t$ such that $\Gamma \vdash_{\mathrm{NuK} *}^{*} A_{t}$ and $\Gamma \vdash_{\mathrm{NuK}_{*}}^{*} \overline{\neg \mathrm{B}_{\mathrm{t}}}$.

$\Gamma$ is a SCAPEGOAT set for $(\rightarrow)_{N_{*}}$ iff for every formula of the form $\neg(A \rightarrow$ B), if $\left.\Gamma \vdash_{\mathrm{NuK}_{*}}^{*} \overline{\neg(A \rightarrow B)}\right)_{0}$ then there is some $t$ such that $\Gamma \vdash_{\mathrm{NuK} *}^{*} A_{t}$ and

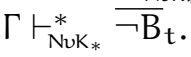

$\mathrm{C}\left(\Gamma^{\prime}\right)$ For $\Gamma$ with unsubscripted formulas and the corresponding $\Gamma_{0}$, we construct $\Gamma^{\prime}$ as follows. Set $\Omega_{0}=\Gamma_{0}$. By $L_{7.3}$, there is an enumeration, $\mathcal{P}_{1}, \mathcal{P}_{2} \ldots$ of all the formulas; let $\mathcal{E}_{0}$ be this enumeration. Then for the first $/ A / s$ in $\mathcal{E}_{i-1}$ such that $s$ is 0 or included in $\Omega_{i-1}$, let $\mathcal{E}_{i}$ be like $\mathcal{E}_{i-1}$ but without $/ A / s$, and set, 
and

$$
\begin{array}{ll}
\Omega_{i}=\Omega_{i-1} & \text { if } \Omega_{i-1} \vdash_{N u x}^{*} \backslash \neg A \backslash_{s} \\
\Omega_{i^{*}}=\Omega_{i-1} \cup\{/ A / s\} & \text { if } \Omega_{i-1} \nvdash_{N u x}^{*} \backslash \neg A \backslash_{s}
\end{array}
$$

$$
\begin{array}{lll}
v K_{4}: & \Omega_{i}=\Omega_{i^{*}} & \text { if } A_{s} \text { is not of the form } / \neg(\mathrm{P} \rightarrow \mathrm{Q}) / \mathrm{s} \\
& \Omega_{\mathrm{i}}=\Omega_{\mathrm{i}^{*}} \cup\left\{\mathrm{P}_{\mathrm{t}}, / \neg \mathrm{Q} / \mathrm{t}\right\} & \text { if } A_{\mathrm{s}} \text { is of the form } / \neg(\mathrm{P} \rightarrow \mathrm{Q}) / \mathrm{s} \\
v N_{4}: & \Omega_{\mathrm{i}}=\Omega_{\mathrm{i}^{*}} & \text { if } A_{\mathrm{s}} \text { is not of the form } / \neg(\mathrm{P} \rightarrow \mathrm{Q}) / \mathrm{o} \\
& \Omega_{\mathrm{i}}=\Omega_{\mathrm{i}^{*}} \cup\left\{\mathrm{P}_{\mathrm{t}}, \neg \mathrm{Q} / \mathrm{t}\right\} & \text { if } A_{\mathrm{s}} \text { is of the form } / \neg(\mathrm{P} \rightarrow \mathrm{Q}) / 0 \\
v K_{*}: & \Omega_{\mathrm{i}}=\Omega_{\mathrm{i}^{*}} & \text { if } A_{\mathrm{s}} \text { is not of the form } / \neg(\mathrm{P} \rightarrow \mathrm{Q}) / \mathrm{s} \\
& \Omega_{\mathrm{i}}=\Omega_{\mathrm{i}^{*}} \cup\left\{\mathrm{P}_{\mathrm{t}}, \neg \overline{\mathrm{Q}}_{\mathrm{t}}\right\} & \text { if } A_{\mathrm{s}} \text { is of the form } / \neg(\mathrm{P} \rightarrow \mathrm{Q}) / \mathrm{s} \\
v N_{*}: & \Omega_{\mathrm{i}}=\Omega_{\mathrm{i}^{*}} & \text { if } A_{\mathrm{s}} \text { is not of the form } \overline{\neg(\mathrm{P} \rightarrow \mathrm{Q})_{0}} \\
& \Omega_{\mathrm{i}}=\Omega_{\mathrm{i}^{*}} \cup\left\{\mathrm{P}_{\mathrm{t}}, \overline{\neg Q}_{\mathrm{t}}\right\} & \text { if } A_{\mathrm{s}} \text { is of the form } \overline{\neg(\mathrm{P} \rightarrow \mathrm{Q})_{0}}
\end{array}
$$

-where $t$ is the first subscript not included in $\Omega_{\mathrm{i}^{*}}$

then

$$
\Gamma^{\prime}=\bigcup_{i \geqslant 0} \Omega_{i}
$$

Note that there is always sure to be a subscript $t$ not in $\Omega_{i^{*}}$ insofar as there are infinitely many subscripts, and at any stage only finitely many formulas are added - the only subscripts in the initial $\Omega_{0}$ being 0 . Suppose $s$ appears in $\Gamma^{\prime}$; then there is some $\Omega_{i}$ in which it is first appears; and any formula $\mathcal{P}_{j}$ in the original enumeration that has subscript $s$ is sure to be "considered" for inclusion at a subsequent stage.

L 7.4 For any s included in $\Gamma^{\prime}, \Gamma^{\prime}$ is s-maximal.

Suppose $s$ is included in $\Gamma^{\prime}$ but $\Gamma^{\prime}$ is not s-maximal. Then there is some $A_{s}$ such that $\Gamma^{\prime} H_{N o x}^{*} / A / s$ and $\Gamma^{\prime} H_{N u x}^{*} \backslash \neg A \backslash_{s}$. For any $i$, each member of $\Omega_{i-1}$ is in $\Gamma^{\prime}$; so if $\Omega_{i-1} \vdash_{\mathrm{Nux}}^{*} \backslash \neg A \backslash_{s}$ then $\Gamma^{\prime} \vdash_{\mathrm{Nux}}^{*} \backslash \neg A \backslash_{s}$; but $\Gamma^{\prime} \nvdash_{\mathrm{NuX}}^{*}$ $\backslash \neg A \backslash_{s}$; so $\Omega_{i-1} \nvdash_{\text {Nux }}^{*} \backslash \neg A \backslash_{s}$; so since $s$ is included in $\Gamma^{\prime}$, there is a stage in the construction that sets $\Omega_{i^{*}}=\Omega_{i-1} \cup\{/ A / s\}$; so by construction, $/ A / s \in \Gamma^{\prime} ;$ so $\Gamma^{\prime} \vdash_{\text {Nox }}^{*} / A / s$. This is impossible; reject the assumption: $\Gamma^{\prime}$ is s-maximal.

L7.5 If $\Gamma_{0}$ is consistent, then each $\Omega_{i}$ is consistent.

Suppose $\Gamma_{0}$ is consistent.

Basis: $\Omega_{0}=\Gamma_{0}$ and $\Gamma_{0}$ is consistent; so $\Omega_{0}$ is consistent.

Assp: For any $i, 0 \leqslant i<k, \Omega_{i}$ is consistent.

Show: $\Omega_{k}$ is consistent.

$\Omega_{k}$ is either (i) $\Omega_{k-1}$, (ii) $\Omega_{k^{*}}=\Omega_{k-1} \cup\{/ A / s\}$, (iii) $\Omega_{k^{*}} \cup$ $\left\{\mathrm{P}_{\mathrm{t}}, / \neg \mathrm{Q} / \mathrm{t}\right\}$ in $v K_{4}$ or $v \mathrm{~N}_{4}$, or (iv) $\Omega_{\mathrm{k}^{*}} \cup\left\{\mathrm{P}_{\mathrm{t}}, \overline{\neg \mathrm{Q}_{\mathrm{t}}}\right\}$ in $v K_{*}$ or $v \mathrm{~N}_{*}$.

(i) Suppose $\Omega_{k}$ is $\Omega_{k-1}$. By assumption, $\Omega_{k-1}$ is consistent; so $\Omega_{k}$ is consistent.

(ii) Suppose $\Omega_{k}$ is $\Omega_{k^{*}}=\Omega_{k-1} \cup\{/ A / s\}$. Then by construction, $s$ is 0 or in $\Omega_{k-1}$ and $\Omega_{k-1} \nvdash_{N u x}^{*} \backslash \neg A \backslash_{s}$; so by L7.2, $\Omega_{k-1} \cup\{/ A / s\}$ is consistent; so $\Omega_{\mathrm{k}}$ is consistent. 
(iii) Suppose $\Omega_{\mathrm{k}}$ is $\Omega_{\mathrm{k}^{*}} \cup\left\{\mathrm{P}_{\mathrm{t}}, / \neg \mathrm{Q} / \mathrm{t}\right\}$ in $v \mathrm{~K}_{4}$ or $v \mathrm{~N}_{4}$. In this case, as above, $\Omega_{\mathrm{k} *}$ is consistent and by construction, $/ \neg(\mathrm{P} \rightarrow \mathrm{Q}) / \mathrm{s} \in$ $\Omega_{k^{*}}$ (in $v N_{4}$, with $s=0$ ). Suppose $\Omega_{k}$ is inconsistent. Then there is some $A_{\mathfrak{u}}$ such that $\Omega_{\mathrm{k}^{*}} \cup\left\{\mathrm{P}_{\mathrm{t}}, / \neg \mathrm{Q} / \mathrm{t}\right\} \vdash_{\mathrm{Nux}}^{*} / / \mathrm{A} / / \mathrm{u}$ and $\Omega_{k^{*}} \cup\left\{P_{t}, / \neg Q / t\right\} \vdash_{\text {Nux }}^{*}\|\neg A\|_{\mathfrak{u}}$. So reason as follows,

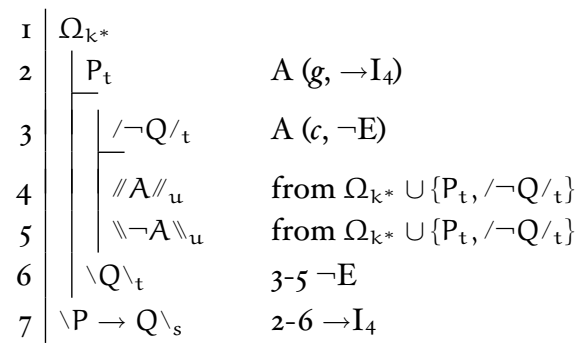

where, by construction, $t$ is not in $\Omega_{k^{*}}$ and for $v N_{4}, s=0$. So $\Omega_{\mathrm{k}^{*}} \vdash_{\mathrm{NuX}}^{*} \backslash \mathrm{P} \rightarrow \mathrm{Q} \backslash_{s}$; but $/ \neg(\mathrm{P} \rightarrow \mathrm{Q}) / \mathrm{s} \in \Omega_{\mathrm{k}^{*}}$; so $\Omega_{\mathrm{k}^{*}} \vdash_{\mathrm{NuX}}^{*}$ $/ \neg(\mathrm{P} \rightarrow \mathrm{Q}) / s$; so $\Omega_{\mathrm{k}^{*}}$ is inconsistent. This is impossible; reject the assumption: $\Omega_{k}$ is consistent.

(iv) Suppose $\Omega_{\mathrm{k}}$ is $\Omega_{\mathrm{k}^{*}} \cup\left\{\mathrm{P}_{\mathrm{t}}, \overline{\neg Q}_{\mathrm{t}}\right\}$ in $v \mathrm{~K}_{*}$ or $v \mathrm{~N}_{*}$. In this case, as above, $\Omega_{\mathrm{k} *}$ is consistent and by construction, $/ \neg(\mathrm{P} \rightarrow \mathrm{Q}) /_{\mathrm{s}} \in$ $\Omega_{\mathrm{k}^{*}}$ (in $v \mathrm{~K}_{*}$, with overline and $s=0$ ). Suppose $\Omega_{\mathrm{k}}$ is inconsistent. Then there is some $A_{u}$ such that $\Omega_{k^{*}} \cup\left\{P_{t}, \overline{\neg Q}_{t}\right\} \vdash_{\text {Nux }}^{*} / / A / / u$ and $\Omega_{k^{*}} \cup\left\{P_{t}, \overline{\neg Q}_{t}\right\} \vdash_{N v X}^{*}\|\neg A\|_{u}$. So reason as follows,

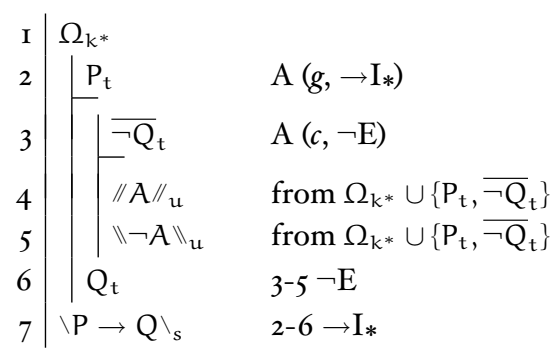

where, by construction, $t$ is not in $\Omega_{k^{*}}$ and for $v N_{*}, \backslash P \rightarrow Q \backslash_{s}$ is without overline and $s=0$. So $\Omega_{\mathrm{k}^{*}} \vdash_{\mathrm{NuX}}^{*} \backslash \mathrm{P} \rightarrow \mathrm{Q} \backslash_{\mathrm{s}}$; but $/ \neg(\mathrm{P} \rightarrow$ $\mathrm{Q}) / s \in \Omega_{\mathrm{k}^{*}}$; so $\Omega_{\mathrm{k}^{*}} \vdash_{\mathrm{Nux}}^{*} / \neg(\mathrm{P} \rightarrow \mathrm{Q}){ }_{\mathrm{s}}$; so $\Omega_{\mathrm{k}^{*}}$ is inconsistent. This is impossible; reject the assumption: $\Omega_{k}$ is consistent.

For any $i, \Omega_{i}$ is consistent.

L7.6 If $\Gamma_{0}$ is consistent, then $\Gamma^{\prime}$ is consistent.

Reasoning parallel to L2.6 and L6.6.

L7.7 If $\Gamma_{0}$ is consistent, then $\Gamma^{\prime}$ is a scapegoat set for $(\rightarrow)_{K_{4}},(\rightarrow)_{N_{4}},(\rightarrow)_{K_{*}}$, and $(\rightarrow)_{\mathrm{N}_{*}}$.

For $(\rightarrow)_{K_{4}}$ and $(\rightarrow)_{N_{4}}$. Suppose $\Gamma_{0}$ is consistent and $\Gamma^{\prime} \vdash_{\mathrm{Nux}}^{*} / \neg(\mathrm{P} \rightarrow$ $\mathrm{Q}) / s$. By L $7.6, \Gamma^{\prime}$ is consistent; and by the constraints on subscripts, $s$ is 
included in $\Gamma^{\prime}$. Since $\Gamma^{\prime}$ is consistent, $\Gamma^{\prime} \nvdash_{\mathrm{Nux}}^{*} \backslash \neg \neg(\mathrm{P} \rightarrow \mathrm{Q}) \backslash_{s}$; so there is a stage in the construction process where $\Omega_{i^{*}}=\Omega_{i-1} \cup\{/ \neg(\mathrm{P} \rightarrow \mathrm{Q}) / \mathrm{s}\}$ and $\Omega_{i}=\Omega_{i^{*}} \cup\left\{P_{t}, / \neg Q / t\right\} ;$ so by construction, $P_{t} \in \Gamma^{\prime}$ and $/ \neg Q / t \in \Gamma^{\prime}$; so $\Gamma^{\prime} \vdash_{\mathrm{Nux}}^{*} \mathrm{P}_{\mathrm{t}}$ and $\Gamma^{\prime} \vdash_{\mathrm{Nux}}^{*} / \neg \mathrm{Q} / \mathrm{t}$. So $\Gamma^{\prime}$ is a scapegoat set for $(\rightarrow)_{\mathrm{K}_{4}}$ and $(\rightarrow)_{N_{4}}$ - where the argument for $(\rightarrow)_{N_{4}}$ assumes $s=0$.

For $(\rightarrow)_{K_{*}}$ and $(\rightarrow)_{N_{*}}$. Suppose $\Gamma_{0}$ is consistent and $\Gamma^{\prime} \vdash_{\mathrm{NuX}}^{*} / \neg(\mathrm{P} \rightarrow$ Q) $/ s$. By L 7.6, $\Gamma^{\prime}$ is consistent; and by the constraints on subscripts, $s$ is included in $\Gamma^{\prime}$. Since $\Gamma^{\prime}$ is consistent, $\Gamma^{\prime} \nvdash_{\mathrm{Nux}}^{*} \backslash \neg \neg(\mathrm{P} \rightarrow \mathrm{Q}) \backslash_{s}$; so there is a stage in the construction process where $\Omega_{i^{*}}=\Omega_{i-1} \cup\{/ \neg(\mathrm{P} \rightarrow \mathrm{Q}) / \mathrm{s}\}$ and $\Omega_{i}=\Omega_{i^{*}} \cup\left\{P_{t}, \overline{\neg Q}_{t}\right\}$; so by construction, $P_{t} \in \Gamma^{\prime}$ and $\overline{\neg Q_{t}} \in \Gamma^{\prime}$; so $\Gamma^{\prime} \vdash_{\mathrm{NuX}}^{*} \mathrm{P}_{\mathrm{t}}$ and $\Gamma^{\prime} \vdash_{\mathrm{Nux}}^{*} \overline{\neg Q}_{\mathrm{t}}$. So $\Gamma^{\prime}$ is a scapegoat set for $(\rightarrow)_{\mathrm{K}_{*}}$ and $(\rightarrow)_{\mathrm{N}_{*}}$ - where the argument for $(\rightarrow)_{N_{*}}$ assumes $/ \neg(P \rightarrow Q) / s$ is with overline and $s=0$.

$\mathrm{C}$ (I) We construct an interpretation $\mathrm{I}=\langle\mathrm{W}, \mathrm{N}, \overline{\mathrm{N}}, \mathrm{h}\rangle$ based on $\Gamma^{\prime}$ as follows.

$v K_{x}$ : For the $K$ systems, let $W$ have a member $w_{s}$ corresponding to each subscript $s$ included in $\Gamma^{\prime}$. Then set $N=\bar{N}=W$ and $h_{w_{s}}(/ p /)=1$ iff $\Gamma^{\prime} \vdash_{\text {Nox }}^{*} / p / s$.

$v N_{4}$ : Let $W$ have a member $w_{s}$ corresponding to each subscript $s$ included in $\Gamma^{\prime}$. Then set $N=\bar{N}=\left\{w_{0}\right\} ; h_{w_{s}}(/ p /)=1$ iff $\Gamma^{\prime} \vdash_{\text {Nox }}^{*}$ $/ p / s$; and for $s \neq 0, h_{w_{s}}(/ A \rightarrow B /)=1$ iff $\Gamma^{\prime} \vdash_{N v x}^{*} / A \rightarrow B / s$.

$v N_{*}$ : Let $W$ have a member $w_{s}$ corresponding to each subscript $s$ included in $\Gamma^{\prime}$. Then set $N=\left\{w_{0}\right\}$ and $\bar{N}=\phi ; h_{w_{s}}(/ p /)=1$ iff $\Gamma^{\prime} \vdash_{\mathrm{Nux}}^{*} / \mathrm{p} /{ }_{s} ; \mathrm{h}_{w_{s}}\left(\overline{\mathrm{P} \rightarrow \mathrm{Q})}=1\right.$ iff $\Gamma^{\prime} \vdash_{\mathrm{Nux}}^{*} \overline{(\mathrm{P} \rightarrow \mathrm{Q})_{s}}$; and for $\mathrm{s} \neq 0$, $h_{w_{s}}(A \rightarrow B)=1$ iff $\Gamma^{\prime} \vdash_{\text {Nux }}^{*}(A \rightarrow B)_{s}$.

L7.8 If $\Gamma_{0}$ is consistent then for $\langle W, N, \bar{N}, h\rangle$ constructed as above, and for any $s$ included in $\Gamma^{\prime}, h_{w_{s}}(/ A /)=1$ iff $\Gamma^{\prime} \vdash_{\mathrm{NuX}}^{*} / A / s$.

Suppose $\Gamma_{0}$ is consistent and $s$ is included in $\Gamma^{\prime}$. By L $7.4, \Gamma^{\prime}$ is s-maximal. By L 7.6 and $\mathrm{L}_{7.7}, \Gamma^{\prime}$ is consistent and a scapegoat set for the different conditionals. Now by induction on the number of operators in $/ A / s$,

Basis: If $/ \mathrm{A} / \mathrm{s}$ has no operators, then it is a parameter $/ \mathrm{p} / \mathrm{s}$ and by construction, $h_{w_{s}}(/ p /)=1$ iff $\Gamma^{\prime} \vdash_{N u x}^{*} / p / s$. So $h_{w_{s}}(/ A /)=1$ iff $\Gamma^{\prime} \vdash_{\text {Nox }}^{*} / \mathrm{A} / \mathrm{s}$.

Assp: For any $i, 0 \leqslant i<k$, if $/ A / s$ has $i$ operators, then $h_{w_{s}}(/ A /)=1$ iff $\Gamma^{\prime} \vdash_{\mathrm{Nux}}^{*} / \mathrm{A} / \mathrm{s}$.

Show: If $/ A / s$ has $k$ operators, then $h_{w_{s}}(/ A /)=1 \mathrm{iff} \Gamma^{\prime} \vdash_{N u X}^{*} / A / s$. If $/ A / s$ has $k$ operators, then it is of the form $/ \neg P / s, / P \wedge Q / s$, $/ \mathrm{P} \vee \mathrm{Q} /{ }_{s}$ or $/ \mathrm{P} \rightarrow \mathrm{Q} / \mathrm{s}$, where $\mathrm{P}$ and $\mathrm{Q}$ have $<\mathrm{k}$ operators.

$(\neg) / A / s$ is $/ \neg P / s$. (i) Suppose $h_{w_{s}}(/ A /)=1$; then $h_{w_{s}}(/ \neg P /)=1$; so by $\mathrm{Hv}(\neg), h_{w_{s}}(\backslash P \backslash)=0$; so by assumption, $\Gamma^{\prime} \nvdash_{\mathrm{Nvx}}^{*} \backslash P \backslash_{s}$; 
so by s-maximality, $\Gamma^{\prime} \vdash_{\mathrm{Nux}}^{*} / \neg \mathrm{P} / \mathrm{s}$, where this is to say, $\Gamma^{\prime} \vdash_{\mathrm{Nox}}^{*}$ $/ A / s$. (ii) Suppose $\Gamma^{\prime} \vdash_{\mathrm{Nux}}^{*} / A / s$; then $\Gamma^{\prime} \vdash_{\mathrm{NuX}}^{*} / \neg P / s$; so by consistency, $\Gamma^{\prime} \nvdash_{N_{u x}}^{*} \backslash P \backslash_{s}$; so by assumption, $h_{w_{s}}(\backslash P \backslash)=0$; so by $\mathrm{H} v(\neg), h_{w_{s}}(/ \neg \mathrm{P} /)=1$, where this is to say, $h_{w_{s}}(/ A /)=1$. So $h_{w_{s}}(/ A /)=1$ iff $\Gamma^{\prime} \vdash_{\text {Nox }}^{*} / A / s$.

$(\wedge)$

$(\mathrm{V})$

$(\rightarrow) / A / s$ is $/ P \rightarrow Q / s$. (i) Suppose $h_{w_{s}}(/ A /)=1$ but $\Gamma^{\prime} H_{N o x}^{*} / A / s$; then $h_{w_{s}}(/ \mathrm{P} \rightarrow \mathrm{Q} /)=1$, but $\Gamma^{\prime} \nvdash_{\mathrm{Nux}}^{*} / \mathrm{P} \rightarrow \mathrm{Q} / s_{s}$; from the latter, by s-maximality, $\Gamma^{\prime} \vdash_{\mathrm{Nux}}^{*} \backslash \neg(\mathrm{P} \rightarrow \mathrm{Q}) \backslash_{s}$.

$v K_{4}$ : In this case, $N=\bar{N}=K$; so $w_{s} \in / N /$. Since $\Gamma^{\prime}$ is a scapegoat set for $(\rightarrow)_{K_{4}}$, there is some $t$ such that $\Gamma^{\prime} \vdash_{\mathrm{NuK}_{4}}^{*}$ $P_{t}$ and $\Gamma^{\prime} \vdash_{\mathrm{NoK}_{4}}^{*} \backslash \neg \mathrm{Q} \backslash_{t}$; from the latter, by consistency, $\Gamma^{\prime} \nvdash_{\mathrm{NuK}_{4}}^{*} / \mathrm{Q} / \mathrm{t}$; so by our assumption, $h_{w_{t}}(\mathrm{P})=1$ and $h_{w_{\mathrm{t}}}(/ \mathrm{Q} /)=0$; so since $w_{\mathrm{s}} \in / \mathrm{N} /$, by $\mathrm{Hv}(\rightarrow)_{4}, \mathrm{~h}_{w_{\mathrm{s}}}(/ \mathrm{P} \rightarrow$ $\left.\mathrm{Q}^{\prime}\right)=0$. This is impossible; reject the assumption: if $\mathrm{h}_{w_{s}}(/ \mathrm{A} /)=1$, then $\Gamma^{\prime} \vdash_{\mathrm{Nux}}^{*} / \mathrm{A} / \mathrm{s}$.

$v N_{4}$ : In this case, when $s=0, w_{s} \in / \mathrm{N} /$ and reasoning is as above. Otherwise, by construction, if $h_{w_{s}}(/ A /)=1$ then $\Gamma^{\prime} \vdash_{\mathrm{Nux}}^{*} / \mathrm{A} / \mathrm{s}$.

$v K_{*}$ : In this case, $N=\bar{N}=K$; so $w_{s} \in / N /$. Since $\Gamma^{\prime}$ is a scapegoat set for $(\rightarrow)_{K_{*}}$, there is some $t$ such that $\Gamma^{\prime} \vdash_{\mathrm{NuK}_{*}}^{*} P_{t}$ and $\Gamma^{\prime} \vdash_{\mathrm{NuK}_{*}}^{*} \neg \bar{Q}_{t}$; from the latter, by consistency, $\Gamma^{\prime} \vdash_{\mathrm{NuK} *}^{*}$ $\mathrm{Q}_{\mathrm{t}}$; so by assumption, $h_{w_{\mathrm{t}}}(\mathrm{P})=1$ and $h_{w_{t}}(\mathrm{Q})=0$; so since $w_{\mathrm{s}} \in / \mathrm{N} /$, by $\mathrm{Hv}(\rightarrow)_{*}, \mathrm{~h}_{w_{s}}(/ \mathrm{P} \rightarrow \mathrm{Q} /)=0$. This is impossible; reject the assumption: if $h_{w_{s}}(/ A /)=1$, then $\Gamma^{\prime} \vdash_{\text {Nox }}^{*} / \mathrm{A} / \mathrm{s}$.

$v N_{*}$ : In this case, when $s=0$ and $/ P \rightarrow Q /$ is without overline - so that $\backslash \neg(\mathrm{P} \rightarrow \mathrm{Q}) \backslash$ is $\overline{\neg(\mathrm{P} \rightarrow \mathrm{Q})}-w_{\mathrm{s}} \in / \mathrm{N} /$ and reasoning is as immediately above. Otherwise, by construction, if $h_{w_{s}}(/ A /)=1$ then $\Gamma^{\prime} \vdash_{N o x}^{*} / A / s$.

So in any of these cases, if $h_{w_{s}}(/ A /)=1$ then $\Gamma^{\prime} \vdash_{\text {Nux }}^{*} / A / s$.

(ii) Suppose $\Gamma^{\prime} \vdash_{\mathrm{Nux}}^{*} / \mathrm{A} / \mathrm{s}$ but $h_{w_{s}}(/ \mathrm{A} /)=0$; then $\Gamma^{\prime} \vdash_{\mathrm{Nux}}^{*} / \mathrm{P} \rightarrow$ $\mathrm{Q} /{ }_{\mathrm{s}}$ but $\mathrm{h}_{w_{\mathrm{s}}}(/ \mathrm{P} \rightarrow \mathrm{Q} /)=0$.

$v K_{4}$ : From the latter, by $\mathrm{H} v(\rightarrow)_{4}$, there is some $w_{\mathrm{t}} \in \mathrm{W}$ such that $h_{w_{t}}(P)=1$ and $h_{w_{t}}(/ Q /)=0$; so by assumption, $\Gamma^{\prime} \vdash_{\mathrm{NuK}_{4}}^{*} P_{\mathrm{t}}$ and $\Gamma^{\prime} \nvdash_{\mathrm{NuK}_{4}}^{*} / \mathrm{Q} / \mathrm{t}$; so by s-maximality, $\Gamma^{\prime} \vdash_{\mathrm{NuK}_{4}}^{*}$ $\neg \neg Q \backslash_{t}$. So by reasoning as follows, 


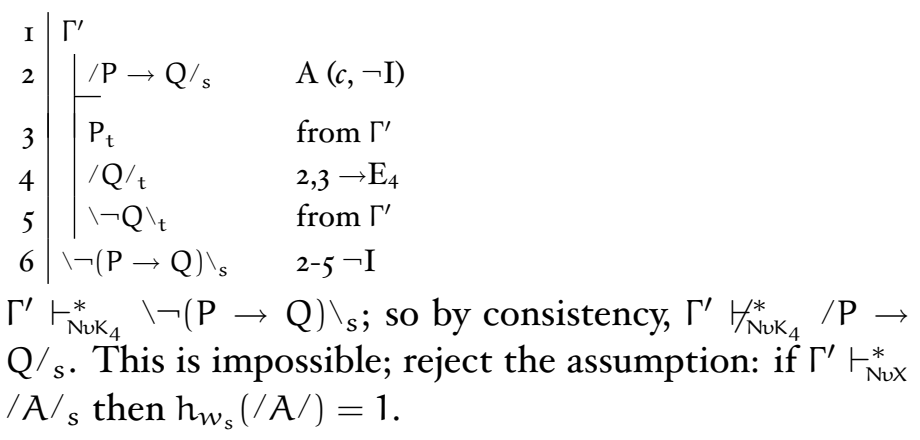

$v N_{4}$ : When $s=0$, the reasoning is as above. Otherwise, by construction, if $\Gamma^{\prime} \vdash_{\text {NuX }}^{*} / A / s$, then $h_{w_{s}}(/ A /)=1$.

$v \mathrm{~K}_{*}$ : From the latter, by $\mathrm{H} v(\rightarrow)_{*}$, there is some $w_{\mathrm{t}} \in \mathrm{W}$ such that $h_{w_{\mathrm{t}}}(/ / \mathrm{P} / /)=1$ and $h_{w_{\mathrm{t}}}(/ / \mathrm{Q} / /)=0$; so by assumption, $\Gamma^{\prime} \vdash_{\mathrm{NuK}_{4}}^{*} / / \mathrm{P} / / \mathrm{t}$ and $\Gamma^{\prime} \nvdash_{\mathrm{NuK}_{4}}^{*} / / \mathrm{Q} / / \mathrm{t}$; so by s-maximality, $\Gamma^{\prime} \vdash_{\mathrm{NuK}_{4}}^{*}\|\neg \mathrm{Q}\|_{t}$. So by reasoning as follows,

\begin{tabular}{l|ll}
$\mathrm{I}$ & $\Gamma^{\prime}$ & \\
2 & $\mid / \mathrm{P} \rightarrow \mathrm{Q} / \mathrm{s}$ & $\mathrm{A}(c, \neg \mathrm{I})$ \\
3 & $\mid / / \mathrm{P} / \mathrm{t}$ & from $\Gamma^{\prime}$ \\
4 & $\| / \mathrm{Q} / \mathrm{t}_{\mathrm{t}}$ & $2,3 \rightarrow \mathrm{E}_{*}$ \\
5 & $\|\neg \mathrm{Q}\|_{\mathrm{t}}$ & from $\Gamma^{\prime}$ \\
6 & $\backslash \neg(\mathrm{P} \rightarrow \mathrm{Q}) \backslash_{\mathrm{s}}$ & $2-5 \neg \mathrm{I}$
\end{tabular}

$\Gamma^{\prime} \vdash_{\mathrm{NuK}_{*}}^{*} \backslash \neg(\mathrm{P} \rightarrow \mathrm{Q}) \backslash_{s}$; so by consistency, $\Gamma^{\prime} \vdash_{\mathrm{NuK}_{*}}^{*} / \mathrm{P} \rightarrow$ $\mathrm{Q} / s$. This is impossible; reject the assumption: if $\Gamma^{\prime} \vdash_{\mathrm{NuX}}^{*}$ $/ A / s$ then $h_{w_{s}}(/ A /)=1$.

$v N_{*}$ : When $s=0$ and $/ P \rightarrow Q /$ is without overline, the reasoning is as immediately above. Otherwise, by construction, if $\Gamma^{\prime} \vdash_{\text {NuX }}^{*} / A / s$ then $h_{w_{s}}(/ A /)=1$.

So in any of these cases, if $\Gamma^{\prime} \vdash_{\text {Nux }}^{*} / A / s$ then $h_{w_{s}}(/ A /)=$ 1. So $h_{w_{s}}(/ A /)=1$ iff $\Gamma^{\prime} \vdash_{\text {Nux }}^{*} / A / s$.

For any $A_{s}, h_{w_{s}}(/ A /)=1$ iff $\Gamma^{\prime} \vdash_{N u x}^{*} / A / s$.

L7.9 If $\Gamma_{0}$ is consistent, then $\langle W, N, \bar{N}, h\rangle$ constructed as above is an $v X$ interpretation.

This is immediate, by construction.

MAP For any $w_{\mathrm{s}} \in \mathrm{W}$, set $\mathrm{m}(\mathrm{s})=w_{\mathrm{s}}$; otherwise $\mathrm{m}(\mathrm{s})$ is arbitrary.

L7.IO If $\Gamma_{0}$ is consistent, then $h_{m}\left(\Gamma_{0}\right)=1$.

Reasoning parallel to L2.IO and L6.9.

Main result: Suppose $\Gamma \models_{v x} A$ but $\Gamma \nvdash_{N v x} A$. Then $\Gamma_{0} \models_{v x}^{*} A_{0}$ but $\Gamma_{0} \nvdash_{N u x}^{*} A_{0}$. By $(\mathrm{DN})$, if $\Gamma_{0} \vdash_{\mathrm{NuX}}^{*} \neg \neg A_{0}$, then $\Gamma_{0} \vdash_{\mathrm{Nux}}^{*} A_{0}$; so $\Gamma_{0} \nvdash_{\mathrm{Nux}}^{*} \neg \neg A_{0}$; so by $L_{7.2}, \Gamma_{0} \cup\left\{\overline{\neg A_{0}}\right\}$ 
is consistent; so by $\mathrm{L}_{7.9}$ and $\mathrm{L} 7 . \mathrm{IO}$, there is an $v X$ interpretation $\langle\mathrm{W}, \mathrm{N}, \overline{\mathrm{N}}, \mathrm{h}\rangle_{\mathrm{m}}$ constructed as above such that $h_{m}\left(\Gamma_{0} \cup\left\{\overline{\neg A}_{0}\right\}\right)=1$; so $h_{m(0)}(\overline{\neg A})=1$; so by $\mathrm{Hv}(\neg), h_{m(0)}(A)=0$; so $h_{m}\left(\Gamma_{0}\right)=1$ and $h_{m(0)}(A)=0$; so by $V v X^{*}$, $\Gamma_{0} \not \nvdash_{v x}^{*} A_{0}$. This is impossible; reject the assumption: if $\Gamma \models_{v x} A$, then $\Gamma \vdash_{\text {Nvx }} A$.

\section{8 mainstream RELEVANT LOGiCs: $B x$ (CH. IO,II)}

The treatment here for Priest's chapter II is minimal: there are only resources for $C K$ first introduced in chapter II, not chapter IO. I abandon the fourvalued approach from previous sections, and follow Priest in developing the star-semantics on its own terms. ${ }^{7}$

\section{I LANGUAGE / SEMANTIC NOTIONS}

LBx The vocabulary consists of propositional parameters $p_{0}, p_{1} \ldots$ with the operators, $\neg, \wedge, \vee, \rightarrow$, (and $>$ ). Each propositional parameter is a FORMULA; if $A$ and $B$ are formulas, so are $\neg A,(A \wedge B),(A \vee B),(A \rightarrow B)$ and $(A>B)$. $A \supset B$ abbreviates $\neg A \vee B$, and $A \equiv B$ abbreviates $(A \supset$ B) $\wedge(B \supset A)$.

IBRX Without ' $>$ ' in the language, an INTERPRETATION is $\langle W, N, R, *, v\rangle$ where $W$ is a set of worlds; $N$ is a subset of $W$; $R$ is a subset of $W^{3}=W \times W \times W$; $*$ is a function from worlds to worlds such that $w^{* *}=w$; and $v$ is a function such that for any $w \in W$ and $p, v_{w}(p)=1$ or $v_{w}(p)=0$. As a constraint on interpretations, we require also,

NC For any $w \in N$, Rwxy iff $x=y$

Where $x$ is empty or indicates some combination of the following constraints,

(C8) If Rabc, then Rac*b*

(C9) If there is an $x$ such that Rabx and Rxcd then there is a $y$ such that Racy and Rbyd

(Cro) If there is an $x$ such that Rabx and Rxcd then there is a $y$ such that Rbcy and Rayd

(CII) If Rabc then Rbac

(CI2) If Rabc then there is an $x$ such that Rabx and Rxbc

(Ci3) If Rabx and Rxcd then Racd

${ }^{7}$ The four-valued approach does apply to some of these logics. But it is complicated considerably (as we have already begun to see with the double normal worlds for $v X_{*}$ of the previous section), and the approach does not apply to all the logics. For details, see [9, 8], and for related derivations along the lines of the four-valued approach from this paper my [II]. As I suggest, this incapacity may be related to motivations for systems like $D W$ which do not transfer naturally to stronger systems like $T W, R W$ and especially $R$. 
(々) If Rabc then (i) if $v_{a}(p)=1$, then $v_{c}(p)=1$, and (ii) if $v_{c^{*}}(p)=1$, then $v_{a^{*}}(p)=1$.

$\langle\mathrm{W}, \mathrm{N}, \mathrm{R}, *, v\rangle$ is a $B x$ interpretation when it meets the constraints from $x$. System $B$ has none of the extra constraints; $B_{D W}$ is $B_{C 8} ; B_{T W}$ is

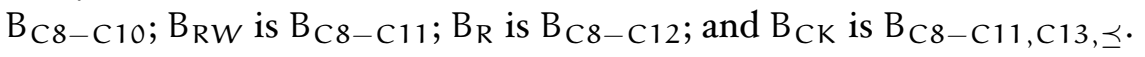

IBCX When ' $>$ ' is in the language, an interpretation is $\left\langle W, N, R,\left\{R_{A} \mid A \in\right.\right.$ $\mathfrak{I}\}, *, v\rangle$, where $\mathfrak{I}$ is the set of all formulas and $\mathrm{R}_{\mathrm{A}}$ is a subset of $\mathrm{W}^{2}$. Condition NC remains in place, but none of $\mathrm{C} 8-\mathrm{Cr}_{3}$ or $(\preceq)$. That is all for $B_{C}$ (what Priest calls $C_{B}$ ). Where $f_{A}(w)=\left\{x \in W \mid w R_{A} x\right\}$, and $[A]=\left\{x \in W \mid v_{w}(A)=1\right\}, B_{C^{+}}$adds the constraints,

(I) For any $w \in N, f_{A}(w) \subseteq[A]$

(2) For any $w \in N$, if $w \in[A]$, then $w \in f_{A}(w)$

TB For complex expressions,

$(\neg) v_{w}(\neg A)=1$ if $v_{w^{*}}(A)=0$, and 0 otherwise.

$(\wedge) v_{w}(A \wedge B)=1$ if $v_{w}(A)=1$ and $v_{w}(B)=1$, and 0 otherwise.

( $\vee v_{w}(A \vee B)=1$ if $v_{w}(A)=1$ or $v_{w}(B)=1$, and 0 otherwise.

$(\rightarrow) v_{w}(\mathrm{~A} \rightarrow \mathrm{B})=1$ iff there are no $x, y \in W$ such that Rwxy and $v_{x}(A)=1$ but $v_{y}(B)=0$.

(>) $v_{w}(\mathrm{~A}>\mathrm{B})=1$ iff there is no $x \in W$ such that $w \mathrm{R}_{\mathrm{A}} x$ and $v_{x}(\mathrm{~B})=$ 0.

For a set $\Gamma$ of formulas, $v_{w}(\Gamma)=1$ iff $v_{w}(A)=1$ for each $A \in \Gamma$; then,

$\operatorname{VBx} \Gamma \models_{B x} A$ iff there is no $B x$ interpretation $\langle W, N, R, *, v\rangle /\left\langle W, N, R,\left\{R_{A} \mid\right.\right.$ $A \in \mathfrak{I}\}, *, v\rangle$ and $w \in \mathrm{N}$ such that $v_{w}(\Gamma)=1$ and $v_{w}(\mathrm{~A})=0$.

\subsection{NATURAL DERIVATIONS: $N B X$}

Allow subscripts of the sort $i$ and $i \#$. Where $s$ is a subscript $i$ or $i^{\#}, \bar{s}$ is the other. Say $s$ is "introduced" as a subscript when either $s$ or $\bar{s}$ is a subscript. For subscripts $s, t, u$ allow also expressions of the sort $s \simeq t$, s.t.u and $A_{s / t}$. Let $\mathcal{P}(s)$ be any expression in which $s$ appears, and $\mathcal{P}(t)$ the same expression with one or more instances of $s$ replaced by $t$.




$\wedge \mathrm{I} \mid \begin{aligned} & \mathrm{P}_{\mathrm{s}} \\ & \mathrm{Q}_{\mathrm{s}} \\ & (\mathrm{P} \wedge \mathrm{Q})_{\mathrm{s}}\end{aligned}$<smiles>[Mg][Mg][Mg]</smiles>

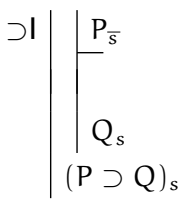<smiles>C#CC(=O)C(=O)O</smiles><smiles>CCCC(C)(C)C(=O)O</smiles>

where $t$ and $u$ are not introduced in any undischarged premise or assumption

$$
\wedge \mathrm{E} \mid \begin{array}{ll|l}
(\mathrm{P} \wedge \mathrm{Q})_{s} & \wedge \mathrm{E} & (\mathrm{P} \wedge \mathrm{Q})_{s} \\
\mathrm{P}_{\mathrm{s}} & \mathrm{Q}_{\mathrm{s}}
\end{array}
$$



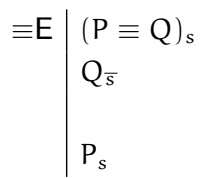

$$
\text { ol } \mid \begin{aligned}
& \mathrm{s} \simeq \mathrm{t} \\
& \text { o.s.t }
\end{aligned}
$$

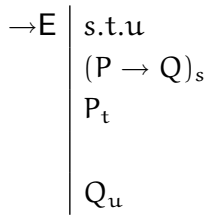<smiles>COC1CCCCC1(C)OC</smiles>

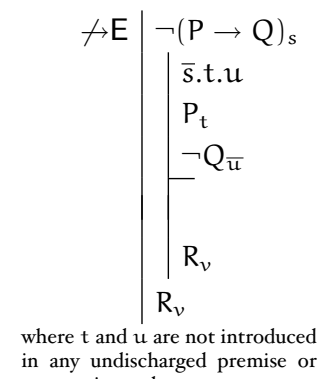
in any undischarged premise or assumption or by $v$

$\simeq \mathrm{E} \mid$\begin{tabular}{l|l}
$\mathrm{s} \simeq \mathrm{t}$ & $\mathrm{s} \simeq \mathrm{t}$ \\
$\mathcal{P}(\mathrm{s})$ & $\mathcal{P}(\overline{\mathrm{s}})$ \\
& \\
$\mathcal{P}(\mathrm{t})$ & $\mathcal{P}(\overline{\mathrm{t}})$
\end{tabular}

These are the rules of $N B$, where $\supset \mathrm{I}, \supset \mathrm{E}, \equiv \mathrm{I}, \equiv \mathrm{E}$ and, as we shall see, $\not \supset \mathrm{I}$ and $\rightarrow \mathrm{E}$ are derived. With $\mathrm{s} \simeq \mathrm{t}$, we can introduce $\mathrm{s} \simeq \mathrm{s}$ by $\simeq \mathrm{I}$, and then get $\mathrm{t} \simeq \mathrm{s}$ by $\simeq \mathrm{E}$; so informally, we let $\simeq \mathrm{E}$ include also a derived rule that reverses order around ' $\simeq$ ' - using $s \simeq \mathrm{t}$ to replace some instance(s) of $\mathrm{t}(\mathrm{t})$ with $\mathrm{s}(\overline{\mathrm{s}})$. As usual, subscripts are 0 or introduced in an assumption that requires new subscripts (and similarly for the following). To make things easier to follow, cite lines for $\rightarrow$ E only in the order listed above: first access, then the conditional, then the antecedent.

For relevant systems $\mathrm{NB}_{x}$, include rules from the following as appropriate. 


\begin{tabular}{|c|c|c|c|c|c|c|c|}
\hline AM8 & $\begin{array}{l}\text { s.t.u } \\
\text { s.u.u. } \bar{t}\end{array}$ & AM9 & \begin{tabular}{|l} 
s.t.x \\
x.u.v \\
$\mid \begin{array}{l}\text { s.u.y } \\
\text { t.y.v }\end{array}$ \\
\\
$P_{w}$ \\
$P_{w}$
\end{tabular} & AM10 & $\begin{array}{l}\text { s.t.x } \\
\text { x.u.v } \\
\mid \begin{array}{l}\text { t.u.y } \\
\text { s.y.v }\end{array} \\
P_{w} \\
P_{w}\end{array}$ & AM11 & $\begin{array}{l}\text { s.t.u } \\
\text { t.s.u }\end{array}$ \\
\hline AM12 & $\begin{array}{l}\text { s.t.u } \\
\mid \begin{array}{l}\text { s.t.y } \\
\text { y.t.u }\end{array}\end{array}$ & AM13 & $\begin{array}{l}\text { s.t.u } \\
\text { u.v.w } \\
\text { s.v.w }\end{array}$ & $\mathrm{AM} \preceq$ & $\mid \begin{array}{l}\text { s.t.u } \\
P_{s} \\
P_{u}\end{array}$ & $\mathrm{AM} \preceq$ & $\begin{array}{l}\text { s.t.u } \\
P_{\bar{u}} \\
P_{\bar{s}}\end{array}$ \\
\hline
\end{tabular}

For AM9, AMıo and AMı2, $y$ is not introduced in any undischarged premise or assumption, or by $w$. Note that the right-hand version of $A M \preceq$ is a derived rule in $\mathrm{NB}_{\mathrm{CK}}$ : from s.t.u it follows by AMII that t.s.u; and from AM8 that t. $\bar{u} . \bar{s}$; so from $A M i r$ that $\bar{u} . t . \bar{s}$; so from $P_{\bar{u}}$ by the left-hand version that $P_{\bar{s}}$.

For the systems $N B_{C_{x}}$ revert to the rules of $N B$. Then add $>\mathrm{I}$ and $>\mathrm{E}$. As we show just below, $\ngtr \mathrm{I}$ and $\ngtr \mathrm{E}$ are derived.
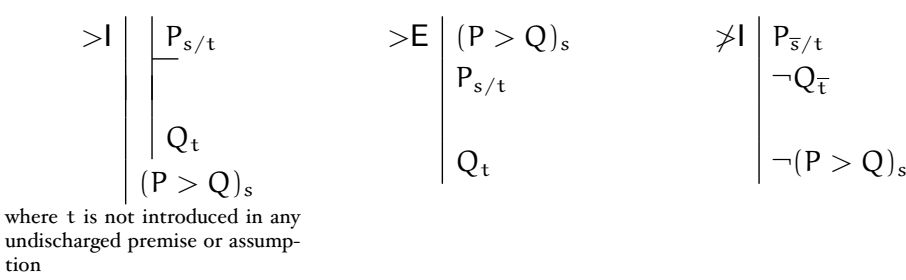

tion

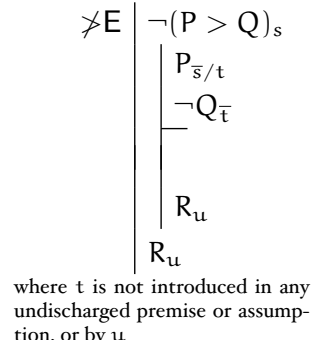

tion, or by u

As before, corresponding to constraints (I) and (2) for the $\mathrm{C}^{+}$system, are AMPI and $\mathrm{AMP}_{2}$, now restricted to apply just at the normal world o.

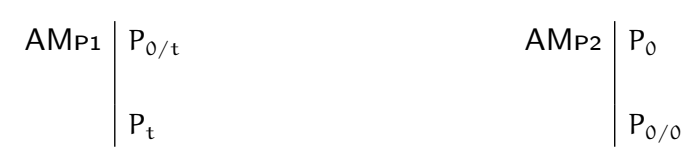

Where $\Gamma$ is a set of unsubscripted formulas, let $\Gamma_{0}$ be those same formulas, each with subscript $\mathrm{o}$. Then,

$\mathrm{NBx} \Gamma \vdash_{\mathrm{NBx}} A$ iff there is an $N B x$ derivation of $A_{0}$ from the members of $\Gamma_{0}$.

Derived rules carry over much as one would expect. Thus, e.g.,

\begin{tabular}{lll|l|ll|l|l}
$\mathrm{MT}$ & $(\mathrm{P} \supset \mathrm{Q})_{s}$ & $\mathrm{NB}$ & $(\mathrm{P} \equiv \mathrm{Q})_{s}$ & $(\mathrm{P} \equiv \mathrm{Q})_{s}$ & $\mathrm{DS}$ & $(\mathrm{P} \vee \mathrm{Q})_{s}$ & $(\mathrm{P} \vee \mathrm{Q})_{s}$ \\
$\neg \mathrm{Q}_{\bar{s}}$ & & $\neg \mathrm{P}_{\bar{s}}$ & $\neg \mathrm{Q}_{\bar{s}}$ & & $\neg \mathrm{P}_{\bar{s}}$ & $\neg \mathrm{Q}_{\bar{s}}$ \\
& & $\neg \mathrm{P}_{s}$ & & & \\
& & $\neg \mathrm{Q}_{s}$ & $\neg \mathrm{P}_{\mathrm{s}}$ & & $\mathrm{Q}_{s}$ & $\mathrm{P}_{\mathrm{s}}$
\end{tabular}


Impl $\quad(\mathrm{P} \supset \mathrm{Q})_{s} \triangleleft \triangleright(\neg \mathrm{P} \vee \mathrm{Q})_{s}$

$(\neg \mathrm{P} \supset \mathrm{Q})_{s} \triangleleft \triangleright(\mathrm{P} \vee \mathrm{Q})_{s}$

As examples, $\not \supset \mathrm{I}, \not \rightarrow \mathrm{E}, \ngtr \mathrm{I}$ and $\ngtr \mathrm{E}$ are derived rules in $\mathrm{NB}_{x}$ and $\mathrm{NB}_{\mathrm{C} x}$.

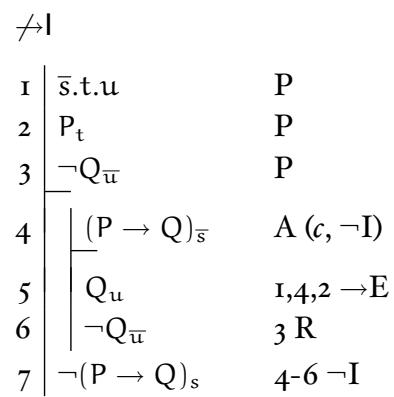

\begin{tabular}{|c|c|c|}
\hline I & $\neg(\mathrm{P} \rightarrow \mathrm{Q})_{\mathrm{s}}$ & $\mathrm{P}$ \\
\hline & $\neg R_{\bar{v}}$ & $\mathrm{~A}(c, \neg \mathrm{E})$ \\
\hline & s.t.u & $\mathrm{A}(\mathrm{g}, \rightarrow \mathrm{I})$ \\
\hline & $P_{t}$ & \\
\hline & $\neg \mathrm{Q}_{\overline{\mathrm{u}}}$ & $\mathrm{A}(c, \neg \mathrm{E})$ \\
\hline & $\vdots$ & with $1,3,4$, \\
\hline & $R_{v}$ & as for $\not \subset \mathrm{E}$ \\
\hline & $\neg R_{\bar{v}}$ & $2 \mathrm{R}$ \\
\hline & $\mathrm{Q}_{u}$ & $5-7 \neg \mathrm{E}$ \\
\hline & $(\mathrm{P} \rightarrow \mathrm{Q})_{\bar{s}}$ & $3^{-8} \rightarrow \mathrm{I}$ \\
\hline & $\neg(\mathrm{P} \rightarrow \mathrm{Q})_{s}$ & I $\mathrm{R}$ \\
\hline & $\mathrm{R}_{v}$ & $2-\mathrm{IO} \neg \mathrm{E}$ \\
\hline
\end{tabular}

\begin{tabular}{|c|c|c|}
\hline \multicolumn{3}{|c|}{$\ngtr 1$} \\
\hline & $\mathrm{P}_{\overline{\mathrm{s}} / \mathrm{t}}$ & $\mathrm{P}$ \\
\hline & $\neg \mathrm{Q}_{\overline{\mathrm{t}}}$ & $\mathrm{P}$ \\
\hline & $(\mathrm{P}>\mathrm{Q})_{\bar{s}}$ & $\mathrm{~A}(c, \neg \mathrm{I})$ \\
\hline & $\mathrm{Q}_{\mathrm{t}}$ & $\mathrm{I}, 3>\mathrm{E}$ \\
\hline & $\neg Q_{\bar{t}}$ & $2 \mathrm{R}$ \\
\hline 5 & $\neg(\mathrm{P}>\mathrm{Q})_{\mathrm{s}}$ & $3-5 \neg \mathrm{I}$ \\
\hline
\end{tabular}

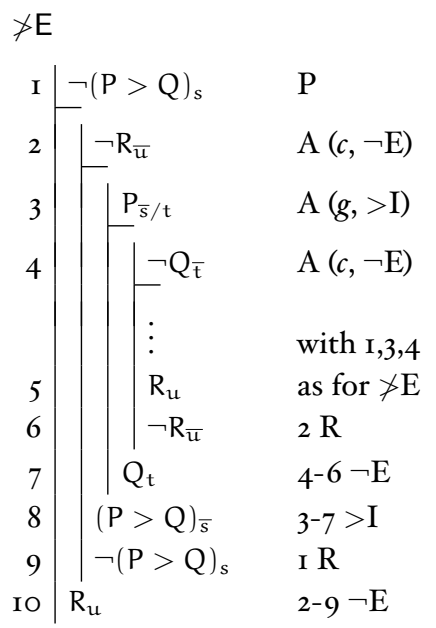

Note the way overlines work (much the way slashes worked before). For $\not \mathrm{E}$, note that the application of $\rightarrow I$ depends on the restriction that $t$ and $u$ are not introduced by $v$; and similarly, for $\ngtr \mathrm{E}$ the application of $>\mathrm{I}$ depends on the restriction that $t$ is not introduced by $u$.

As further examples, here are a few key results that parallel ones from Priest's text. 
$\mathrm{A}_{3} \vdash_{\mathrm{NBx}}(\mathrm{A} \wedge \mathrm{B}) \rightarrow \mathrm{A}$

\begin{tabular}{|c|c|c|}
\hline I & 0.1 .2 & $\mathrm{~A}(g, \rightarrow \mathrm{I})$ \\
\hline 2 & $(A \wedge B)_{1}$ & \\
\hline 3 & $A_{1}$ & $2 \wedge \mathrm{E}$ \\
\hline 4 & $1 \simeq 2$ & I oE \\
\hline 5 & $A_{2}$ & $3,4 \simeq \mathrm{E}$ \\
\hline 6 & {$[(A \wedge B) \rightarrow A]_{0}$} & $\mathrm{I}-5 \rightarrow \mathrm{I}$ \\
\hline
\end{tabular}

A5 $\vdash_{\mathrm{NBx}}[(\mathrm{A} \rightarrow \mathrm{B}) \wedge(\mathrm{A} \rightarrow \mathrm{C})] \rightarrow[\mathrm{A} \rightarrow(\mathrm{B} \wedge \mathrm{C})]$

\begin{tabular}{|c|c|c|}
\hline I & 0.1 .2 & $\mathrm{~A}(g, \rightarrow \mathrm{I})$ \\
\hline 2 & {$[(A \rightarrow B) \wedge(A \rightarrow C)]_{1}$} & \\
\hline 3 & 2.3.4 & $\mathrm{A}(g, \rightarrow \mathrm{I})$ \\
\hline 4 & $\mathrm{~A}_{3}$ & \\
\hline 5 & $1 \simeq 2$ & I oE \\
\hline 6 & 1.3.4 & $3,5 \simeq \mathrm{E}$ \\
\hline 7 & $(A \rightarrow B)_{1}$ & $2 \wedge \mathrm{E}$ \\
\hline 8 & $(A \rightarrow C)_{1}$ & $2 \wedge \mathrm{E}$ \\
\hline 9 & $\mathrm{~B}_{4}$ & $6,7,4 \rightarrow \mathrm{E}$ \\
\hline & $\mathrm{C}_{4}$ & $6,8,4 \rightarrow \mathrm{E}$ \\
\hline II & $(B \wedge C)_{4}$ & $9, \mathrm{IO} \wedge \mathrm{I}$ \\
\hline 12 & {$[A \rightarrow(B \wedge C)]_{2}$} & 3-II $\rightarrow \mathrm{I}$ \\
\hline 3 & $[(A \rightarrow B) \wedge(A \rightarrow C)] \rightarrow[A \rightarrow(B \wedge C)])_{0}$ & $\mathrm{I}-\mathrm{I} 2 \rightarrow \mathrm{I}$ \\
\hline
\end{tabular}

\begin{tabular}{|c|c|c|}
\hline I & $(A \rightarrow \neg B)_{0}$ & $\mathrm{P}$ \\
\hline 2 & 0.1 .2 & $\mathrm{~A}(\mathrm{~g}, \rightarrow \mathrm{I})$ \\
\hline 3 & $\mathrm{~B}_{1}$ & \\
\hline 4 & $\mathrm{~A}_{2 \#}$ & $\mathrm{~A}(c, \neg \mathrm{I})$ \\
\hline 5 & $2^{\#} \simeq 2^{\#}$ & $\simeq \mathrm{I}$ \\
\hline 6 & $0.2^{\#} .2^{\#}$ & $5 \mathrm{OI}$ \\
\hline 7 & $\neg \mathrm{B}_{2 \#}$ & $6, \mathrm{I}, 4 \rightarrow \mathrm{E}$ \\
\hline 8 & $1 \simeq 2$ & $2 \mathrm{oE}$ \\
\hline 9 & $\mathrm{~B}_{2}$ & $3,8 \simeq \mathrm{E}$ \\
\hline & $\neg A_{2}$ & $4^{-9} \neg \mathrm{I}$ \\
\hline II & $(B \rightarrow \neg A)_{0}$ & $2-\mathrm{IO} \rightarrow \mathrm{I}$ \\
\hline
\end{tabular}




\begin{tabular}{|c|c|c|}
\hline \multicolumn{3}{|c|}{ A9 $\vdash_{\mathrm{NB}_{\mathrm{T} W}}(\mathrm{~A} \rightarrow \mathrm{B}) \rightarrow[(\mathrm{B} \rightarrow \mathrm{C}) \rightarrow(\mathrm{A} \rightarrow \mathrm{C})]$} \\
\hline I & 0.1 .2 & \multirow[t]{2}{*}{$\mathrm{A}(g, \rightarrow \mathrm{I})$} \\
\hline 2 & $(A \rightarrow B)_{1}$ & \\
\hline 3 & 2.3.4 & \multirow[t]{2}{*}{$\mathrm{A}(g, \rightarrow \mathrm{I})$} \\
\hline 4 & $(\mathrm{~B} \rightarrow \mathrm{C})_{3}$ & \\
\hline 5 & 4.5.6 & \multirow[t]{2}{*}{$\mathrm{A}(g, \rightarrow \mathrm{I})$} \\
\hline 6 & $A_{5}$ & \\
\hline 7 & $1 \simeq 2$ & $\mathrm{I} O \mathrm{E}$ \\
\hline 8 & $(\mathrm{~A} \rightarrow \mathrm{B})_{2}$ & $2,7 \simeq \mathrm{E}$ \\
\hline 9 & | 2.5.7 & \multirow[t]{2}{*}{$\mathrm{A}(g, 3,5$ AM9) } \\
\hline IO & 3.7 .6 & \\
\hline II & $\mathrm{B}_{7}$ & $9,8,6 \rightarrow \mathrm{E}$ \\
\hline $\mathrm{I} 2$ & $\mathrm{C}_{6}$ & $\mathrm{IO}, 4, \mathrm{II} \rightarrow \mathrm{E}$ \\
\hline 13 & $\mathrm{C}_{6}$ & 3,5,9-I2 AM9 \\
\hline I4 & $(A \rightarrow C)_{4}$ & $5^{-\mathrm{I} 3} \rightarrow \mathrm{I}$ \\
\hline 15 & {$[(B \rightarrow C) \rightarrow(A \rightarrow C)]_{2}$} & $3-\mathrm{I} 4 \rightarrow \mathrm{I}$ \\
\hline 16 & $((\mathrm{~A} \rightarrow \mathrm{B}) \rightarrow[(\mathrm{B} \rightarrow \mathrm{C}) \rightarrow(\mathrm{A} \rightarrow \mathrm{C})])_{0}$ & $\mathrm{I}-\mathrm{I} 5 \rightarrow \mathrm{I}$ \\
\hline \multicolumn{3}{|c|}{$\vdash_{\mathrm{NB}_{\mathrm{R}}}(\neg \mathrm{A} \rightarrow \mathrm{A}) \rightarrow \mathrm{A}$} \\
\hline I & 0.1 .2 & $\mathrm{~A}(g, \rightarrow \mathrm{I})$ \\
\hline 2 & $(\neg A \rightarrow A)_{1}$ & \\
\hline 3 & $\neg A_{2 \#}$ & $\mathrm{~A}(c, \neg \mathrm{E})$ \\
\hline 4 & $0.2^{\#} .1 \#$ & I AM8 \\
\hline 5 & $0.2^{\#} .3$ & $\mathrm{~A}(g, 4 \mathrm{AM} \mathbf{2})$ \\
\hline 6 & $3.2^{\#} .1^{\#}$ & \\
\hline 7 & 3.1 .2 & 6 AM8 \\
\hline 8 & 1.3.2 & 7 AMII \\
\hline 9 & $1.2^{\#} .3^{\#}$ & 8 AM8 \\
\hline IO & $A_{3 \#}$ & $9,2,3 \rightarrow \mathrm{E}$ \\
\hline II & $2^{\#} \simeq 3$ & $5 \mathrm{oE}$ \\
\hline $\mathrm{I} 2$ & $A_{2}$ & $\mathrm{IO}, \mathrm{II} \simeq \mathrm{E}$ \\
\hline $\mathrm{I} 3$ & $\mathrm{~A}_{2}$ & 4,5-I2 AMI2 \\
\hline I4 & $\neg A_{2 \#}$ & $3 \mathrm{R}$ \\
\hline I5 & $A_{2}$ & $3-\mathrm{I} 4 \neg \mathrm{E}$ \\
\hline I6 & {$[(\neg A \rightarrow A) \rightarrow A]_{0}$} & $\mathrm{I}-\mathrm{I} 5 \rightarrow \mathrm{I}$ \\
\hline
\end{tabular}




\begin{tabular}{|c|c|c|}
\hline \multicolumn{3}{|c|}{$\mathrm{AI}_{3} \vdash_{\mathrm{NB}_{\mathrm{CK}}} \mathrm{A} \rightarrow(\mathrm{B} \rightarrow \mathrm{A})$} \\
\hline I & 0.1 .2 & $\mathrm{~A}(g, \rightarrow \mathrm{I})$ \\
\hline 2 & $A_{1}$ & \\
\hline 3 & 2.3 .4 & $\mathrm{~A}(g, \rightarrow \mathrm{I})$ \\
\hline 4 & $B_{3}$ & \\
\hline 5 & 1.0 .2 & I AMri \\
\hline 6 & 1.3.4 & 5,3 $\mathrm{AM}_{3} 3$ \\
\hline 7 & $\mathrm{~A}_{4}$ & 2,6 AM $\preceq$ \\
\hline 8 & $(B \rightarrow A)_{2}$ & 3-7 $\rightarrow \mathrm{I}$ \\
\hline 9 & {$[A \rightarrow(B \rightarrow A)]_{0}$} & $\mathrm{I}-8 \rightarrow \mathrm{I}$ \\
\hline \multicolumn{3}{|c|}{$\vdash_{\mathrm{NB}_{\mathrm{CK}}}(\mathrm{A} \vee \mathrm{B}) \rightarrow((\mathrm{A} \rightarrow \mathrm{B}) \rightarrow \mathrm{B})$} \\
\hline I & 0.1 .2 & $\mathrm{~A}(g, \rightarrow \mathrm{I})$ \\
\hline 2 & $(A \vee B)_{1}$ & \\
\hline 3 & | 2.3 .4 & $\mathrm{~A}(g, \rightarrow \mathrm{I})$ \\
\hline 4 & $(A \rightarrow B)_{3}$ & \\
\hline 5 & $A_{1}$ & $\mathrm{~A}(g, 2 \vee \mathrm{E})$ \\
\hline 6 & $1 \simeq 2$ & $\mathrm{I} O \mathrm{E}$ \\
\hline 7 & $A_{2}$ & $5,6 \simeq \mathrm{E}$ \\
\hline 8 & 3.2 .4 & 3 AMrI \\
\hline 9 & $\mathrm{~B}_{4}$ & $8,4,7 \rightarrow \mathrm{E}$ \\
\hline IO & $\mathrm{B}_{1}$ & $\mathrm{~A}(g, 2 \vee \mathrm{E})$ \\
\hline II & 1.0 .2 & I AMrI \\
\hline $\mathrm{I} 2$ & 1.3 .4 & II,3 AMI3 \\
\hline I3 & $\mathrm{B}_{4}$ & IO,I2 $\mathrm{AM} \preceq$ \\
\hline I4 & $\mathrm{B}_{4}$ & $2,5-9, \mathrm{IO}-\mathrm{I} 3 \vee \mathrm{E}$ \\
\hline I5 & $((\mathrm{A} \rightarrow \mathrm{B}) \rightarrow \mathrm{B})_{2}$ & $3-\mathrm{I} 4 \rightarrow \mathrm{I}$ \\
\hline I6 & {$[(A \vee B) \rightarrow((A \rightarrow B) \rightarrow B)]_{0}$} & $\mathrm{I}-\mathrm{I} 5 \rightarrow \mathrm{I}$ \\
\hline
\end{tabular}

\subsection{SOUNDNESS AND COMPLETENESS}

Preliminaries: Begin with generalized notions of validity. For a model $\langle W, N, R$, $*, v\rangle$ or $\left\langle W, N, R,\left\{R_{A} \mid A \in \mathfrak{I}\right\}, *, v\right\rangle$, let $m$ be a map from subscripts into $W$ such that $m(0) \in N$ and $m(\bar{s})=m(s)^{*}$. Say $\langle W, N, R, *, v\rangle_{m}$ and $\left\langle W, N, R,\left\{R_{A} \mid A \in\right.\right.$ $\mathfrak{I}\}, *, v\rangle_{m}$ are $\langle\mathrm{W}, \mathrm{N}, \mathrm{R}, *, v\rangle$ and $\left\langle\mathrm{W}, \mathrm{N}, \mathrm{R},\left\{\mathrm{R}_{\mathrm{A}} \mid \mathrm{A} \in \mathfrak{I}\right\}, *, v\right\rangle$ with map $\mathrm{m}$. Then, where $\Gamma$ is a set of expressions of our language for derivations, $v_{m}(\Gamma)=1$ iff for each $A_{s} \in \Gamma, v_{m(s)}(A)=1$, for each $s \simeq t \in \Gamma, m(s)=m(t)$, for each s.t.u $\in \Gamma$, $\langle\mathrm{m}(\mathrm{s}), \mathrm{m}(\mathrm{t}), \mathrm{m}(\mathrm{u})\rangle \in \mathrm{R}$, and for each $A_{s / t} \in \Gamma,\langle\mathrm{m}(\mathrm{s}), \mathrm{m}(\mathrm{t})\rangle \in \mathrm{R}_{\mathrm{A}}$. Unless otherwise noted, reasoning is meant to be neutral between interpretations of the different types. Now expand notions of validity to include subscripted formulas, and alternate expressions as indicated in double brackets.

$\mathrm{VBx} * \Gamma \models_{B x}^{*} A_{s} \llbracket s \simeq t / s . t . u / A_{s / t} \rrbracket$ iff there is no $B x$ interpretation with map $m$ where $v_{\mathrm{m}}(\Gamma)=1$ but $v_{\mathrm{m}(\mathrm{s})}(A)=0 \llbracket \mathrm{m}(\mathrm{s}) \neq \mathrm{m}(\mathrm{t}) /\langle\mathrm{m}(\mathrm{s}), \mathrm{m}(\mathrm{t}), \mathrm{m}(\mathrm{u})\rangle \notin$ 


$$
\mathrm{R} /\langle\mathrm{m}(\mathrm{s}), \mathrm{m}(\mathrm{t})\rangle \notin \mathrm{R}_{\mathrm{A}} \rrbracket .
$$

$\mathrm{NB} x^{*} \Gamma \vdash_{\mathrm{NBx}}^{*} A_{s} \llbracket s \simeq \mathrm{t} /$ s.t.u $/ A_{s / t} \rrbracket$ iff there is an $N B x$ derivation of $A_{s}$ $\llbracket s \simeq \mathrm{t} / \mathrm{s} . \mathrm{t} . \mathrm{u} / A_{\mathrm{s} / \mathrm{t}} \rrbracket$ from the members of $\Gamma$.

These notions reduce to the standard ones when all the members of $\Gamma$ and $A$ have subscript 0 (and so are not of the sort $s \simeq t$, s.t.u or $A_{s / t}$ ). For the following, cases omitted are like ones worked, and so left to the reader.

THEOREM 8.I NBx is sound: If $\Gamma \vdash_{\mathrm{NBx}}$ A then $\Gamma \models_{\mathrm{BX}}$ A.

L8.I If $\Gamma \subseteq \Gamma^{\prime}$ and $\Gamma \models_{\mathrm{Bx}}^{*} \mathrm{P}_{\mathrm{s}} \llbracket s \simeq \mathrm{t} /$ s.t.u $/ A_{\mathrm{s} / \mathrm{t}} \rrbracket$, then $\Gamma^{\prime} \models_{\mathrm{Bx}}^{*} \mathrm{P}_{\mathrm{s}} \llbracket s \simeq$ $\mathrm{t} / \mathrm{s} . \mathrm{t} . \mathrm{u} / \mathrm{A}_{\mathrm{s} / \mathrm{t}} \rrbracket$.

Suppose $\Gamma \subseteq \Gamma^{\prime}$ and $\Gamma \models_{B x}^{*} P_{s} \llbracket s \simeq t /$ s.t.u $/ A_{s / t} \rrbracket$, but $\Gamma^{\prime} \nvdash_{B x}^{*} P_{s} \llbracket s \simeq t /$ s.t.u $/ A_{s / t} \rrbracket$. From the latter, by $V B x^{*}$, there is some $B x$ interpretation with $v$ and $\mathrm{m}$ such that $v_{\mathrm{m}}\left(\Gamma^{\prime}\right)=1$ but $v_{\mathrm{m}(\mathrm{s})}(\mathrm{P})=0 \llbracket \mathrm{m}(\mathrm{s}) \neq \mathrm{m}(\mathrm{t}) /$ $\langle\mathrm{m}(\mathrm{s}), \mathrm{m}(\mathrm{t}), \mathrm{m}(\mathrm{u})\rangle \notin \mathrm{R} /\langle\mathrm{m}(\mathrm{s}), \mathrm{m}(\mathrm{t})\rangle \notin \mathrm{R}_{\mathrm{A}} \rrbracket$. But since $v_{\mathrm{m}}\left(\Gamma^{\prime}\right)=1$ and $\Gamma \subseteq \Gamma^{\prime}, v_{\mathrm{m}}(\Gamma)=1$; so $v_{\mathrm{m}}(\Gamma)=1$ but $v_{\mathrm{m}(\mathrm{s})}(\mathrm{P})=0 \llbracket \mathrm{m}(\mathrm{s}) \neq \mathrm{m}(\mathrm{t}) /$ $\langle\mathrm{m}(\mathrm{s}), \mathrm{m}(\mathrm{t}), \mathrm{m}(\mathrm{u})\rangle \notin \mathrm{R} /\langle\mathrm{m}(\mathrm{s}), \mathrm{m}(\mathrm{t})\rangle \notin \mathrm{R}_{\mathrm{A}} \rrbracket ;$ so by $\mathrm{VBx}^{*}, \Gamma \nvdash_{B x}^{*} \mathrm{P}_{\mathrm{s}}$ $\llbracket s \simeq t /$ s.t.u $/ A_{s / t} \rrbracket$. This is impossible; reject the assumption: if $\Gamma \subseteq \Gamma^{\prime}$ and $\Gamma \models_{B x}^{*} P_{s} \llbracket s \simeq t /$ s.t.u $/ A_{s / t} \rrbracket$, then $\Gamma^{\prime} \models_{B x}^{*} P_{s} \llbracket s \simeq t /$ s.t.u $/ A_{s / t} \rrbracket$.

Main result: For each line in a derivation let $\mathcal{P}_{i}$ be the expression on line $i$ and $\Gamma_{i}$ be the set of all premises and assumptions whose scope includes line $i$. We set out to show "generalized" soundness: if $\Gamma \vdash_{\mathrm{NBx}}^{*} \mathcal{P}$ then $\Gamma \models_{\mathrm{Bx}}^{*} \mathcal{P}$. As above, this reduces to the standard result when $\mathcal{P}$ and all the members of $\Gamma$ are formulas with subscript 0 . Suppose $\Gamma \vdash_{\mathrm{NBx}}^{*} \mathcal{P}$. Then there is a derivation of $\mathcal{P}$ from premises in $\Gamma$ where $\mathcal{P}$ appears under the scope of the premises alone. By induction on line number of this derivation, we show that for each line $i$ of this derivation, $\Gamma_{i} \models_{B x}^{*} \mathcal{P}_{i}$. The case when $\mathcal{P}_{i}=\mathcal{P}$ is the desired result.

Basis: $\mathcal{P}_{1}$ is a premise or an assumption $A_{s} \llbracket s \simeq t /$ s.t.u $/ A_{s / t} \rrbracket$. Then $\Gamma_{1}=\left\{A_{s}\right\}$ $\llbracket\{s \simeq \mathrm{t}\} /\{\mathrm{s.t.u}\} /\left\{A_{\mathrm{s} / \mathrm{t}}\right\} \rrbracket ;$; so for any $B x$ interpretation with its $v$ and $\mathrm{m}$, $v_{\mathfrak{m}}\left(\Gamma_{1}\right)=1$ iff $v_{\mathfrak{m}(s)}(A)=1 \llbracket m(s)=m(t) /\langle m(s), m(t), m(u)\rangle \in R /$ $\langle\mathrm{m}(\mathrm{s}), \mathrm{m}(\mathrm{t})\rangle \in \mathrm{R}_{\mathrm{A}} \rrbracket$; so there is no $B x$ interpretation with $v$ and $\mathrm{m}$ such that $v_{\mathrm{m}}\left(\Gamma_{1}\right)=1$ but $v_{\mathrm{m}(\mathrm{s})}(\mathrm{A})=0 \llbracket \mathrm{m}(\mathrm{s}) \neq \mathrm{m}(\mathrm{t}) /\langle\mathrm{m}(\mathrm{s}), \mathrm{m}(\mathrm{t}), \mathrm{m}(\mathrm{u})\rangle \notin$ $\mathrm{R} /\langle\mathrm{m}(\mathrm{s}), \mathrm{m}(\mathrm{t})\rangle \notin \mathrm{R}_{A} \rrbracket$. So by $\mathrm{VBx}^{*}, \Gamma_{1} \models_{B x}^{*} A_{s} \llbracket s \simeq \mathrm{t} /$ s.t.u $/ A_{s / t} \rrbracket$, where this is just to say, $\Gamma_{1} \models_{\mathrm{Bx}}^{*} \mathcal{P}_{1}$.

Assp: For any $i, 1 \leqslant i<k, \Gamma_{i} \models_{B x}^{*} \mathcal{P}_{i}$.

Show: $\Gamma_{\mathrm{k}} \models_{\mathrm{Bx}}^{*} \mathcal{P}_{\mathrm{k}}$.

$\mathcal{P}_{k}$ is either a premise, an assumption, or arises from previous lines by $\mathrm{R}, \wedge \mathrm{I}, \wedge \mathrm{E}, \vee \mathrm{I}, \vee \mathrm{E}, \neg \mathrm{I}, \neg \mathrm{E}, \rightarrow \mathrm{I}, \rightarrow \mathrm{E}, \simeq \mathrm{I}, \simeq \mathrm{E}$, oI, oE or, depending on the system, AM8, AM9, AMro, AMır, AMı2, AMı3, AM $\preceq,>I,>E$, $\mathrm{AMP}$, or $\mathrm{AMP}_{2}$. If $\mathcal{P}_{k}$ is a premise or an assumption, then as in the basis, $\Gamma_{\mathrm{k}} \models_{\mathrm{Bx}}^{*} \mathcal{P}_{\mathrm{k}}$. So suppose $\mathcal{P}_{\mathrm{k}}$ arises by one of the rules. 
$(\neg \mathrm{I})$ If $\mathcal{P}_{k}$ arises by $\neg \mathrm{I}$, then the picture is like this,

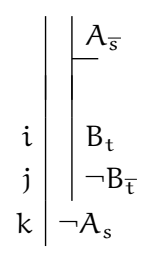

where $i, j<k$ and $\mathcal{P}_{k}$ is $\neg A_{s}$. By assumption, $\Gamma_{i} \models_{B x}^{*} B_{t}$ and $\Gamma_{j} \models_{B x}^{*} \neg B_{\bar{t}}$; but by the nature of access, $\Gamma_{i} \subseteq \Gamma_{k} \cup\left\{A_{\bar{s}}\right\}$ and $\Gamma_{j} \subseteq \Gamma_{k} \cup\left\{A_{\bar{s}}\right\}$; so by L8.I, $\Gamma_{\mathrm{k}} \cup\left\{A_{\bar{s}}\right\} \models_{\mathrm{Bx}}^{*} B_{\mathrm{t}}$ and $\Gamma_{\mathrm{k}} \cup\left\{A_{\bar{s}}\right\} \models_{\mathrm{Bx}}^{*} \neg B_{\overline{\mathrm{t}}}$. Suppose $\Gamma_{\mathrm{k}} \forall_{\mathrm{Bx}}^{*} \neg A_{s}$; then by $\mathrm{VBx}^{*}$, there is a $B x$ interpretation with $v$ and $\mathrm{m}$ such that $v_{\mathrm{m}}\left(\Gamma_{\mathrm{k}}\right)=1$ but $v_{\mathrm{m}(\mathrm{s})}(\neg \mathrm{A})=0$; so by $\mathrm{TB}(\neg), v_{\mathrm{m}(s)^{*}}(\mathrm{~A})=1$; so by the construction of $m, v_{m(\bar{s})}(A)=1$; so $v_{m}\left(\Gamma_{k}\right)=1$ and $v_{m(\bar{s})}(A)=1$; so $v_{m}\left(\Gamma_{k} \cup\right.$ $\left.\left\{\mathrm{A}_{\bar{s}}\right\}\right)=1$; so by $\mathrm{VBx}^{*}, v_{\mathrm{m}(\mathrm{t})}(\mathrm{B})=1$ and $v_{\mathrm{m}(\overline{\mathrm{t}})}(\neg \mathrm{B})=1$; from the latter, by $\mathrm{TB}(\neg), v_{\mathrm{m}(\overline{\mathrm{t}})^{*}}(\mathrm{~B})=0$; so by the construction of $\mathrm{m}, v_{\mathrm{m}(\mathrm{t})}(\mathrm{B})=0$. This is impossible; reject the assumption: $\Gamma_{k} \models_{B x}^{*} \neg A_{s}$, which is to say, $\Gamma_{\mathrm{k}} \models_{\mathrm{Bx}}^{*} \mathcal{P}_{\mathrm{k}}$.

$(\neg \mathrm{E})$

$(\rightarrow \mathrm{I})$ If $\mathcal{P}_{k}$ arises by $\rightarrow \mathrm{I}$, then the picture is like this,

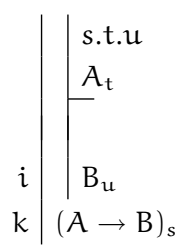

where $i<k, t, u$ are not introduced in any member of $\Gamma_{k}$ (in any undischarged premise or assumption), and $\mathcal{P}_{k}$ is $(A \rightarrow B)_{s}$. By assumption, $\Gamma_{i} \models_{B x}^{*} B_{u}$; but by the nature of access, $\Gamma_{i} \subseteq \Gamma_{k} \cup\left\{\right.$ s.t.u, $\left.A_{t}\right\}$; so by L8.I, $\Gamma_{k} \cup\left\{\right.$ s.t.u, $\left.A_{t}\right\} \models_{B x}^{*} B_{u}$. Suppose $\Gamma_{k} \nvdash_{B x}^{*}(A \rightarrow B)_{s}$; then by VBx*, there is a $B x$ interpretation with $W, R, v$ and $m$ such that $v_{m}\left(\Gamma_{k}\right)=1$ but $v_{\mathrm{m}(\mathrm{s})}(\mathrm{A} \rightarrow \mathrm{B})=0$; so by $\mathrm{TB}(\rightarrow)$, there are $x, y \in W$ such that $\operatorname{Rm}(s) x y$ and $v_{x}(A)=1$ but $v_{y}(B)=0$. Now consider a map $m^{\prime}$ like $m$ except that $\mathrm{m}^{\prime}(\mathrm{t})=\mathrm{x}, \mathrm{m}^{\prime}(\overline{\mathrm{t}})=\mathrm{x}^{*}, \mathrm{~m}^{\prime}(\mathrm{u})=\mathrm{y}$, and $\mathrm{m}^{\prime}(\overline{\mathrm{u}})=\mathrm{y}^{*}$; since $\mathrm{t}$ and $\mathrm{u}$ (along with $\bar{t}$ and $\bar{u}$ ) do not appear in $\Gamma_{k}$, it remains that $v_{m^{\prime}}\left(\Gamma_{k}\right)=1$; 
since $v_{x}(A)=1, v_{m^{\prime}(t)}(A)=1$; and since $R m(s) x y$, with $m(s)=m^{\prime}(s)$, we have $\left\langle m^{\prime}(s), m^{\prime}(t), m^{\prime}(u)\right\rangle \in R$; so $v_{m^{\prime}}\left(\Gamma_{k} \cup\left\{\right.\right.$ s.t.u, $\left.\left.A_{t}\right\}\right)=1$; so by $V_{B x}^{*}, v_{m^{\prime}(u)}(B)=1$. But $m^{\prime}(u)=y$; so $v_{y}(B)=1$. This is impossible; reject the assumption: $\Gamma_{k} \models_{B x}^{*}(A \rightarrow B)_{s}$, which is to say, $\Gamma_{k} \models_{B x}^{*} \mathcal{P}_{k}$.

$(\rightarrow \mathrm{E})$ If $\mathcal{P}_{k}$ arises by $\rightarrow \mathrm{E}$, then the picture is like this,

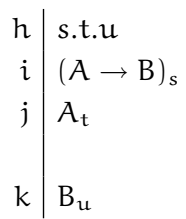

where $h, i, j<k$ and $\mathcal{P}_{k}$ is $B_{u}$. By assumption, $\Gamma_{h} \models_{B x}^{*}$ s.t.u, $\Gamma_{i} \models_{B x}^{*}(A \rightarrow$ $B)_{s}$ and $\Gamma_{j} \models_{B x}^{*} A_{t}$; but by the nature of access, $\Gamma_{h} \subseteq \Gamma_{k}, \Gamma_{i} \subseteq \Gamma_{k}$ and $\Gamma_{j} \subseteq \Gamma_{k}$; so by L8.I, $\Gamma_{k} \models_{B x}^{*}$ s.t.u, $\Gamma_{k} \models_{B x}^{*}(A \rightarrow B)_{s}$ and $\Gamma_{k} \models_{B x}^{*} A_{t}$. Suppose $\Gamma_{\mathrm{k}} \nvdash_{\mathrm{Bx}}^{*} \mathrm{~B}_{\mathrm{u}}$; then by $\mathrm{VBx}^{*}$, there is some $B x$ interpretation with $W$, $R, v$ and $m$ such that $v_{m}\left(\Gamma_{k}\right)=1$ but $v_{m(u)}(B)=0$; since $v_{m}\left(\Gamma_{k}\right)=1$, by $V_{B x}^{*},\langle m(s), m(t), m(u)\rangle \in R, v_{m(s)}(A \rightarrow B)=1$ and $v_{m(t)}(A)=1$; since $v_{m(s)}(A \rightarrow B)=1$, by $\mathrm{TB}(\rightarrow)$, there are no $x, y \in W$ such that $R m(s) x y$ and $v_{x}(A)=1$ but $v_{y}(B)=0$; so since $\langle m(s), m(t), m(u)\rangle \in R$, it is not the case that $v_{m(t)}(A)=1$ and $v_{m(u)}(B)=0$. This is impossible; reject the assumption: $\Gamma_{k} \models_{B x}^{*} B_{u}$, which is to say, $\Gamma_{k} \models_{B x}^{*} \mathcal{P}_{k}$.

$(\simeq \mathrm{I})$ If $\mathcal{P}_{\mathrm{k}}$ arises by $\simeq \mathrm{I}$, then the picture is like this,

$k \mid s \simeq s$

where $\mathcal{P}_{\mathrm{k}}$ is $\mathrm{s} \simeq \mathrm{s}$. Suppose $\Gamma_{\mathrm{k}} \forall_{\mathrm{Bx}}^{*} \mathrm{~s} \simeq \mathrm{s}$; then by $\mathrm{VBx}{ }^{*}$, there is a $B x$ interpretation with $v$, and $\mathrm{m}$ such that $v_{\mathrm{m}}\left(\Gamma_{\mathrm{k}}\right)=1$ but $\mathrm{m}(\mathrm{s}) \neq \mathrm{m}(\mathrm{s})$. This is impossible; reject the assumption: $\Gamma_{\mathrm{k}} \models_{\mathrm{Bx}}^{*} s \simeq s$, which is to say, $\Gamma_{\mathrm{k}} \models_{\mathrm{Bx}}^{*} \mathcal{P}_{\mathrm{k}}$.

( $\simeq \mathrm{E})$ If $A_{k}$ arises by $\simeq \mathrm{E}$, then the picture is like this,

\begin{tabular}{r|llr|l}
$\mathrm{i}$ & $\mathrm{s} \simeq \mathrm{t}$ & & $\mathrm{i}$ & $\mathrm{s} \simeq \mathrm{t}$ \\
$\mathrm{j}$ & $\mathcal{A}(\mathrm{s})$ & or & $\mathrm{k}$ & $\mathcal{A}(\overline{\mathrm{s}})$ \\
$\mathrm{k}$ & $\mathcal{A}(\mathrm{t})$ & & $\mathrm{k}$ & $\mathcal{A}(\overline{\mathrm{t}})$
\end{tabular}

where $i, j<k$ and $\mathcal{P}_{k}$ is $\mathcal{A}(\mathrm{t})$ or $\mathcal{A}(\overline{\mathrm{t}})$. By assumption, $\Gamma_{\mathrm{i}} \models_{\mathrm{Bx}}^{*} \mathrm{~s} \simeq \mathrm{t}$ and $\Gamma_{\mathrm{j}} \models_{\mathrm{Bx}}^{*} \mathcal{A}(\mathrm{s}) / \mathcal{A}(\overline{\mathrm{s}}) ;$ but by the nature of access, $\Gamma_{\mathrm{i}} \subseteq \Gamma_{\mathrm{k}}$ and $\Gamma_{\mathrm{j}} \subseteq \Gamma_{\mathrm{k}}$; so by L8.I, $\Gamma_{\mathrm{k}} \models_{\mathrm{Bx}}^{*} \mathrm{~s} \simeq \mathrm{t}$ and $\Gamma_{\mathrm{k}} \models_{\mathrm{Bx}}^{*} \mathcal{A}(\mathrm{s}) / \mathcal{A}(\overline{\mathrm{s}})$. In the right-hand case, $\mathcal{A}(\bar{s})$ is of the sort, $A_{\mathfrak{u}}, \mathfrak{u} \simeq v, u . v . w$ or $A_{\mathfrak{u} / v}$ where one of $u, v$, or $w$ is $\overline{\mathrm{s}}$. Suppose $\mathcal{A}(\overline{\mathrm{s}})$ is $A_{\overline{\mathrm{s}}}$ and $\Gamma_{\mathrm{k}} \nvdash_{\mathrm{Bx}}^{*} A_{\overline{\mathrm{t}}}$. Then by $\mathrm{VBx}{ }^{*}$, there is some $B x$ interpretation with $v$ and $m$ such that $v_{m}\left(\Gamma_{k}\right)=1$ but $v_{m(\bar{t})}(A)=0$. Since $v_{m}\left(\Gamma_{k}\right)=1$, by VBx* $m(s)=m(t)$ and $v_{m(\bar{s})}(A)=1$; since $m(s)=m(t), m(s)^{*}=m(t)^{*}$; but by the construction of $m, m(s)^{*}=$ 
$\mathfrak{m}(\overline{\mathrm{s}})$ and $\mathfrak{m}(\mathrm{t})^{*}=\mathfrak{m}(\overline{\mathrm{t}})$; so $\mathfrak{m}(\overline{\mathrm{s}})=\mathfrak{m}(\overline{\mathrm{t}})$; so $v_{\mathfrak{m}(\overline{\mathrm{t}})}(\mathrm{A})=1$. This is impossible; reject the assumption: $\Gamma_{\mathrm{k}} \models_{\mathrm{Bx}}^{*} A_{\overline{\mathrm{t}}}$, which is to say, $\Gamma_{\mathrm{k}} \models_{\mathrm{Bx}}^{*} \mathcal{P}_{\mathrm{k}}$. And similarly in the other cases.

(oI) If $\mathcal{P}_{k}$ arises by oI, then the picture is like this,

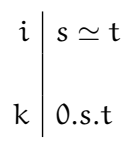

where $i<k$ and $\mathcal{P}_{k}$ is 0 .s.t. By assumption, $\Gamma_{i} \models_{B x}^{*} s \simeq \mathrm{t}$; but by the nature of access, $\Gamma_{\mathrm{i}} \subseteq \Gamma_{\mathrm{k}}$; so by L8.I, $\Gamma_{k} \models_{B x}^{*} \mathrm{~s} \simeq \mathrm{t}$. Suppose $\Gamma_{\mathrm{k}} \not \nvdash_{\mathrm{Bx}}^{*}$ 0.s.t; then by $\mathrm{VBx}^{*}$, there is a $B x$ interpretation with $W, \mathrm{~N}, \mathrm{R}, v$ and $m$ such that $v_{m}\left(\Gamma_{k}\right)=1$ but $\langle m(0), m(s), m(t)\rangle \notin R$; since $v_{m}\left(\Gamma_{k}\right)=1$, by $\mathrm{VBx}^{*}, \mathrm{~m}(\mathrm{~s})=\mathrm{m}(\mathrm{t})$; and by the construction of $\mathrm{m}, \mathrm{m}(0) \in \mathrm{N}$; so by NC, $\langle m(0), m(s), m(t)\rangle \in R$. This is impossible; reject the assumption: $\Gamma_{\mathrm{k}} \models_{\mathrm{Bx}}^{*}$ 0.s.t, which is to say, $\Gamma_{\mathrm{k}} \models_{\mathrm{Bx}}^{*} \mathcal{P}_{\mathrm{k}}$.

(oE) If $\mathcal{P}_{k}$ arises by oE, then the picture is like this,

$$
\begin{array}{l|l}
i & 0 . s . t \\
k & s \simeq t
\end{array}
$$

where $i<k$ and $\mathcal{P}_{k}$ is $s \simeq t$. By assumption, $\Gamma_{i} \models_{B x}^{*}$ 0.s.t; but by the nature of access, $\Gamma_{i} \subseteq \Gamma_{k}$; so by L8.I, $\Gamma_{k} \models_{B x}^{*}$ 0.s.t. Suppose $\Gamma_{k} \nvdash_{B x}^{*}$ $\mathrm{s} \simeq \mathrm{t}$; then by $\mathrm{VBx}^{*}$, there is a $B x$ interpretation with $W, \mathrm{~N}, \mathrm{R}, v$ and $m$ such that $v_{m}\left(\Gamma_{k}\right)=1$ but $m(s) \neq m(t)$; since $v_{m}\left(\Gamma_{k}\right)=1$, by $\mathrm{VBx}^{*},\langle\mathrm{~m}(0), \mathrm{m}(\mathrm{s}), \mathrm{m}(\mathrm{t})\rangle \in \mathrm{R}$; and by the construction of $\mathrm{m}, \mathrm{m}(0) \in \mathrm{N}$; so by $\mathrm{NC}, \mathrm{m}(\mathrm{s})=\mathrm{m}(\mathrm{t})$. This is impossible; reject the assumption: $\Gamma_{\mathrm{k}} \models_{\mathrm{Bx}}^{*} \mathrm{~s} \simeq \mathrm{t}$, which is to say, $\Gamma_{\mathrm{k}} \models_{\mathrm{Bx}}^{*} \mathcal{P}_{\mathrm{k}}$.

(AM8) If $\mathcal{P}_{k}$ arises by AM8, then the picture is like this,

$$
\begin{array}{l|l}
i & s . t . u \\
k & s . \bar{u} . \bar{t}
\end{array}
$$

where $i<k$ and $\mathcal{P}_{k}$ is s.u.t.t. Where this rule is included in $N B x, B x$ includes condition C8. By assumption, $\Gamma_{\mathrm{i}} \models_{\mathrm{Bx}}^{*}$ s.t.u; but by the nature of access, $\Gamma_{i} \subseteq \Gamma_{k}$; so by L8.I, $\Gamma_{k} \models_{B x}^{*}$ s.t.u. Suppose $\Gamma_{k} \nvdash_{B x}^{*}$ s.u. $\bar{t}$; then by $\mathrm{VBx}^{*}$, there is a $B x$ interpretation with $\mathrm{R}, v$ and $\mathrm{m}$ such that $v_{m}\left(\Gamma_{k}\right)=1$ but $\langle m(s), m(\bar{u}), m(\bar{t})\rangle \notin R$; since $v_{m}\left(\Gamma_{k}\right)=1$, by $V_{B x}^{*}$, $\langle m(s), m(t), m(u)\rangle \in R$; so by $C 8,\left\langle m(s), m(u)^{*}, m(t)^{*}\right\rangle \in R$; so by the construction of $m,\langle m(s), m(\bar{u}), m(\bar{t})\rangle \in R$. This is impossible; reject the assumption: $\Gamma_{\mathrm{k}} \models_{\mathrm{B} x}^{*}$ s. $\bar{u} . \overline{\mathrm{t}}$, which is to say, $\Gamma_{\mathrm{k}} \models_{\mathrm{B} x}^{*} \mathcal{P}_{\mathrm{k}}$.

(AM9) If $\mathcal{P}_{k}$ arises by $\mathrm{AM}_{9}$, then the picture is like this, 


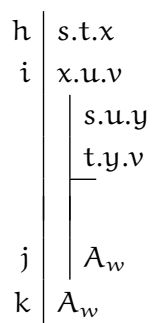

where $h, i, j<k, y$ is not introduced in any member of $\Gamma_{k}$ (in any undischarged premise or assumption) or by $w$, and $\mathcal{P}_{k}$ is $A_{w}$. Where this rule is included in $N B x, B x$ includes condition C9. By assumption, $\Gamma_{\mathrm{h}} \models_{B x}^{*}$ s.t.x, $\Gamma_{\mathrm{i}} \models_{\mathrm{Bx}}^{*}$ x.u.v and $\Gamma_{\mathrm{j}} \models_{\mathrm{Bx}}^{*} A_{w}$; but by the nature of access, $\Gamma_{\mathrm{h}} \subseteq \Gamma_{\mathrm{k}}$, $\Gamma_{i} \subseteq \Gamma_{k}$ and $\Gamma_{j} \subseteq \Gamma_{k} \cup\{$ s.u.y, t.y.v $\}$; so by L8.I, $\Gamma_{k} \models_{B x}^{*}$ s.t.x, $\Gamma_{k} \models_{B x}^{*}$ x.u.v and $\Gamma_{\mathrm{k}} \cup\{$ s.u.y, t.y.v $\} \models_{\mathrm{Bx}}^{*} A_{w}$. Suppose $\Gamma_{\mathrm{k}} \nvdash_{\mathrm{Bx}}^{*} A_{w}$; then by VBx*, there is a $B x$ interpretation with $W, R, v$ and $m$ such that $v_{m}\left(\Gamma_{k}\right)=1$ but $v_{m(w)}(A)=0$; since $v_{m}\left(\Gamma_{k}\right)=1$, by $V_{B x}^{*},\langle m(s), m(t), m(x)\rangle \in R$ and $\langle\mathrm{m}(\mathrm{x}), \mathfrak{m}(\mathrm{u}), \mathfrak{m}(v)\rangle \in \mathrm{R}$; so by $\mathrm{C}_{9}$, there is some $z \in \mathrm{W}$ such that $\langle\mathrm{m}(\mathrm{s}), \mathrm{m}(\mathrm{u}), z\rangle \in \mathrm{R}$ and $\langle\mathrm{m}(\mathrm{t}), z, \mathrm{~m}(v)\rangle \in \mathrm{R}$. Now consider a map $\mathrm{m}^{\prime}$ like $m$ except that $\mathrm{m}^{\prime}(\mathrm{y})=z$ and $\mathrm{m}^{\prime}(\bar{y})=z^{*}$; since $y$ (along with $\bar{y}$ ) does not appear in $\Gamma_{k}$, it remains that $v_{m^{\prime}}\left(\Gamma_{k}\right)=1$; and since $m(s)=m^{\prime}(s)$, and similarly for $\mathrm{t}, \mathrm{u}$ and $v,\left\langle\mathrm{~m}^{\prime}(\mathrm{s}), \mathrm{m}^{\prime}(\mathrm{u}), \mathrm{m}^{\prime}(\mathrm{y})\right\rangle \in \mathrm{R}$ and $\left\langle\mathrm{m}^{\prime}(\mathrm{t}), \mathrm{m}^{\prime}(\mathrm{y})\right.$, $\left.\mathrm{m}^{\prime}(v)\right\rangle \in R$; so $v_{\mathrm{m}^{\prime}}\left(\Gamma_{\mathrm{k}} \cup\{\right.$ s.u.y, t.y. $\left.v\}\right)=1$; so by $\mathrm{VBx}^{*}, v_{\mathrm{m}^{\prime}(w)}(\mathrm{A})=1$. But since $y$ is not introduced by $w, \mathfrak{m}^{\prime}(w)=\mathfrak{m}(w)$; so $v_{\mathfrak{m}(w)}(A)=1$. This is impossible; reject the assumption: $\Gamma_{k} \models_{B x}^{*} A_{w}$, which is to say, $\Gamma_{\mathrm{k}} \models_{\mathrm{Bx}}^{*} \mathcal{P}_{\mathrm{k}}$.

(AMio)

(AMII)

(AMı)

$\left(\mathrm{AM}_{1}\right)^{2}$ If $\mathcal{P}_{k}$ arises by $\mathrm{AMr}_{3}$, then the picture is like this,

\begin{tabular}{l|l}
$\mathfrak{i}$ & s.t.u \\
$\mathbf{j}$ & u.v.w \\
$\mathrm{k}$ & s.v.w
\end{tabular}

where $i, j<k$ and $\mathcal{P}_{k}$ is s.v.w. Where this rule is included in $N B x$, $B x$ includes condition Crz. By assumption, $\Gamma_{\mathrm{i}} \models_{B x}^{*}$ s.t.u and $\Gamma_{\mathrm{j}} \models_{B x}^{*}$ u.v.w; but by the nature of access, $\Gamma_{i} \subseteq \Gamma_{k}$ and $\Gamma_{j} \subseteq \Gamma_{k}$; so by L8.I, $\Gamma_{\mathrm{k}} \models_{\mathrm{Bx}}^{*}$ s.t.u and $\Gamma_{\mathrm{j}} \models_{\mathrm{B}_{\mathrm{x}}}^{*}$ u.v.w. Suppose $\Gamma_{\mathrm{k}} \not \models_{\mathrm{Bx}}^{*}$ s.v.w; then by $\mathrm{VBx}^{*}$, there is a $B x$ interpretation with $R, v$ and $m$ such that $v_{m}\left(\Gamma_{k}\right)=1$ but $\langle\mathrm{m}(\mathrm{s}), \mathrm{m}(v), \mathrm{m}(w)\rangle \notin \mathrm{R}$; since $v_{\mathrm{m}}\left(\Gamma_{\mathrm{k}}\right)=1$, by $\mathrm{VBx}^{*}$, we have $\langle\mathrm{m}(\mathrm{s}), \mathrm{m}(\mathrm{t}), \mathrm{m}(\mathrm{u})\rangle \in \mathrm{R}$ and $\langle\mathrm{m}(\mathrm{u}), \mathrm{m}(v), \mathrm{m}(w)\rangle \in \mathrm{R}$; and so by $\mathrm{Cr}_{3}$, $\langle\mathrm{m}(\mathrm{s}), \mathrm{m}(v), \mathrm{m}(w)\rangle \in \mathrm{R}$. This is impossible; reject the assumption: $\Gamma_{\mathrm{k}} \models_{\mathrm{B} x}^{*}$ s.v.w, which is to say, $\Gamma_{\mathrm{k}} \models_{\mathrm{B} x}^{*} \mathcal{P}_{\mathrm{k}}$. 
(AM $\preceq$ ) If $\mathcal{P}_{k}$ arises by $\mathrm{AM} \preceq$, then the picture is like this,

$$
\begin{array}{r|l}
i & \text { s.t.u } \\
j & A_{s} \\
k & A_{u}
\end{array}
$$

where $i, j<k$ and $\mathcal{P}_{k}$ is $A_{u}$. In $B C K$, where this rule is included in $N B x$, $B x$ includes condition $(\preceq)$ along with $\mathrm{C} 8, \mathrm{CII}_{\mathrm{I}}$ and $\mathrm{Cr}_{3}$. By assumption, $\Gamma_{i} \models_{B x}^{*}$ s.t.u and $\Gamma_{j} \models_{B x}^{*} A_{s}$; but by the nature of access, $\Gamma_{i} \subseteq \Gamma_{k}$ and $\Gamma_{j} \subseteq \Gamma_{k}$; so by L8.I, $\Gamma_{k} \models_{B x}^{*}$ s.t.u and $\Gamma_{k} \models_{B x}^{*} A_{s}$. Suppose $\Gamma_{k} \forall_{B x}^{*} A_{u}$; then by $\mathrm{VBx}^{*}$, there is a $B x$ interpretation with $v$ and $\mathrm{m}$ such that $v_{\mathrm{m}}\left(\Gamma_{\mathrm{k}}\right)=1$ but $v_{m(u)}(A)=0$; since $v_{m}\left(\Gamma_{k}\right)=1$, by VBx*, $\langle m(s), m(t), m(u)\rangle \in$ $R$ and $v_{m(s)}(A)=1$; But given Rabc, under current constraints, (i) if $v_{\mathrm{a}}(A)=1$ then $v_{\mathrm{c}}(A)=1$ and (ii) if $v_{\mathrm{c}^{*}}(A)=1$ then $v_{\mathrm{a}^{*}}(A)=1$. Suppose Rabc.

Basis: $A$ is a parameter $p$. (i) Suppose $v_{a}(A)=1$; then $v_{a}(p)=1$; so by $(\preceq), v_{\mathrm{c}}(p)=1$; so $v_{\mathrm{c}}(A)=1$. (ii) Suppose $v_{\mathrm{c}^{*}}(A)=1$; then $\nu_{\mathrm{c}^{*}}(\mathrm{p})=1$; so by $(\preceq), v_{\mathrm{a}^{*}}(\mathrm{p})=1$; so $\nu_{\mathrm{a}^{*}}(A)=1$.

Assp: For any $i, 0 \leqslant i<k$, if $A$ has $i$ operator symbols then (i) if $v_{\mathrm{a}}(A)=1$ then $v_{\mathrm{c}}(A)=1$ and (ii) if $v_{\mathrm{c}^{*}}(\mathrm{~A})=1$ then $v_{\mathrm{a}^{*}}(\mathrm{~A})=1$.

Show: If $A$ has $k$ operator symbols then (i) if $v_{a}(A)=1$ then $v_{c}(A)=1$ and (ii) if $v_{\mathrm{c}^{*}}(\mathrm{~A})=1$ then $v_{\mathrm{a}^{*}}(\mathrm{~A})=1$.

In this system we do not have $>$ in the language. So if $A$ has $k$ operator symbols, it is of the form $\neg \mathrm{P}, \mathrm{P} \wedge \mathrm{Q}, \mathrm{P} \vee \mathrm{Q}$, or $\mathrm{P} \rightarrow \mathrm{Q}$ where $P$ and $Q$ have $<k$ operator symbols.

$(\neg) \mathrm{A}$ is $\neg \mathrm{P}$. (i) Suppose $v_{\mathrm{a}}(\mathrm{A})=1$; then $v_{\mathrm{a}}(\neg \mathrm{P})=1$; so by $\mathrm{TB}(\neg)$, $v_{\mathrm{a}^{*}}(\mathrm{P})=0$; so by assumption, $v_{\mathrm{c}^{*}}(\mathrm{P})=0$; so by $\mathrm{TB}(\neg), v_{\mathrm{c}}(\neg \mathrm{P})=$ 1 ; so $v_{\mathrm{c}}(\mathrm{A})=1$. (ii) Suppose $v_{\mathrm{c}^{*}}(\mathrm{~A})=1$; then $v_{\mathrm{c}^{*}}(\neg \mathrm{P})=1$; so by $\mathrm{TB}(\neg), v_{\mathrm{c}}(\mathrm{P})=0$; so by assumption, $v_{\mathrm{a}}(\mathrm{P})=0$; so by $\mathrm{TB}(\neg)$, $v_{\mathrm{a}^{*}}(\neg \mathrm{P})=1$; so $v_{\mathrm{a}^{*}}(A)=1$.

( $\wedge$ ) $A$ is $P \wedge Q$. (i) Suppose $v_{a}(A)=1$; then $v_{a}(P \wedge Q)=1$; so by $\mathrm{TB}(\wedge), v_{\mathrm{a}}(\mathrm{P})=1$ and $v_{\mathrm{a}}(\mathrm{Q})=1$; so by assumption, $v_{\mathrm{c}}(\mathrm{P})=1$ and $v_{\mathrm{c}}(\mathrm{Q})=1$; so by $\mathrm{TB}(\wedge), v_{\mathrm{c}}(\mathrm{P} \wedge \mathrm{Q})=1$; so $v_{\mathrm{c}}(\mathrm{A})=1$. (ii) Suppose $v_{\mathrm{c}^{*}}(\mathrm{~A})=1$; then $v_{\mathrm{c}^{*}}(\mathrm{P} \wedge \mathrm{Q})=1$; so by $\mathrm{TB}(\wedge), v_{\mathrm{c}^{*}}(\mathrm{P})=1$ and $v_{\mathrm{c}^{*}}(\mathrm{Q})=1$; so by assumption, $v_{\mathrm{a}^{*}}(\mathrm{P})=1$ and $v_{\mathrm{a}^{*}}(\mathrm{Q})=1$; so by $\mathrm{TB}(\wedge), v_{\mathrm{a}^{*}}(\mathrm{P} \wedge \mathrm{Q})=1$; so $v_{\mathrm{a}^{*}}(\mathrm{~A})=1$.

$(\mathrm{V})$

$(\rightarrow) A$ is $P \rightarrow Q$. (i) Suppose $v_{a}(A)=1$ but $v_{c}(A)=0$; then $v_{a}(P \rightarrow$ $\mathrm{Q})=1$ but $\nu_{\mathrm{c}}(\mathrm{P} \rightarrow \mathrm{Q})=0$. From the latter, by $\mathrm{TB}(\rightarrow)$, there are $w, x \in W$ such that Rcwx and $v_{w}(\mathrm{P})=1$ but $v_{x}(\mathrm{Q})=0$. From the former, by $\mathrm{TB}(\rightarrow)$, there are no $y, z \in W$ such that Rayz and $v_{y}(\mathrm{P})=1$ but $v_{z}(\mathrm{Q})=0$. But since Rabc and Rcwx, by $\mathrm{C}_{3}$, 
Rawx; so it is not the case that $v_{w}(\mathrm{P})=1$ and $v_{x}(\mathrm{Q})=0$. This is impossible; reject the assumption: if $v_{a}(A)=1$, then $v_{c}(A)=1$.

(ii) Suppose $v_{\mathrm{c}^{*}}(\mathrm{~A})=1$ but $v_{\mathrm{a}^{*}}(\mathrm{~A})=0$; then $v_{\mathrm{c}^{*}}(\mathrm{P} \rightarrow \mathrm{Q})=1$ but $v_{\mathrm{a}^{*}}(\mathrm{P} \rightarrow \mathrm{Q})=0$. From the latter, by $\mathrm{TB}(\rightarrow)$, there are $w, x \in \mathrm{W}$ such that $\mathrm{Ra}^{*} w x$ and $v_{w}(\mathrm{P})=1$ but $v_{x}(\mathrm{Q})=0$. From the former, by $\mathrm{TB}(\rightarrow)$, there are no $y, z \in W$ such that $\mathrm{Rc}^{*} y z$ and $\nu_{y}(\mathrm{P})=1$ but $v_{z}(\mathrm{Q})=0$. But since Rabc, by CiI, Rbac; so by C8, Rbc*a*; so by $\mathrm{CII}_{1}, \mathrm{Rc}^{*} \mathrm{ba}^{*}$; so with $\mathrm{Ra}^{*} w x$, by $\mathrm{CI}_{3}, \mathrm{Rc}^{*} w x$; so it is not the case that $v_{w}(\mathrm{P})=1$ and $v_{x}(\mathrm{Q})=0$. This is impossible; reject the assumption: if $v_{\mathrm{c}^{*}}(A)=1$, then $v_{\mathrm{a}^{*}}(A)=1$.

For any $A$, (i) if $v_{a}(A)=1$ then $v_{c}(A)=1$ and (ii) if $v_{c^{*}}(A)=1$ then $v_{a^{*}}(A)=1$.

So, returning to the main case, $v_{m(u)}(A)=1$. This is impossible; reject the assumption: $\Gamma_{k} \models_{B x}^{*} A_{u}$, which is to say, $\Gamma_{k} \models_{B x}^{*} \mathcal{P}_{k}$.

$(>\mathrm{I})$

$(>\mathrm{E})$ If $\mathcal{P}_{\mathrm{k}}$ arises by $>\mathrm{E}$, then the picture is like this,

\begin{tabular}{l|l}
$\mathfrak{i}$ & $(\mathrm{A}>\mathrm{B})_{s}$ \\
$\mathfrak{j}$ & $A_{s / t}$ \\
$k$ & $B_{t}$
\end{tabular}

where $i, j<k$ and $\mathcal{P}_{k}$ is $B_{t}$. By assumption, $\Gamma_{i} \models_{B x}^{*}(A>B)_{s}$ and $\Gamma_{j} \models_{B x}^{*}$ $A_{s / t}$; but by the nature of access, $\Gamma_{i} \subseteq \Gamma_{k}$ and $\Gamma_{j} \subseteq \Gamma_{k}$; so by L8.I, $\Gamma_{k} \models_{B x}^{*}(A>B)_{s}$ and $\Gamma_{k} \models_{B x}^{*} A_{s / t}$. Suppose $\Gamma_{k} \forall_{B x}^{*} B_{t}$; then by VBx*, there is some $B x$ interpretation with $W,\left\{R_{A} \mid A \in \mathfrak{I}\right\}, v$ and $m$ such that $v_{m}\left(\Gamma_{k}\right)=1$ but $v_{m(t)}(B)=0$; since $v_{m}\left(\Gamma_{k}\right)=1$, by $V_{B x}^{*}, v_{m(s)}(A>$ B) $=1$ and $\langle m(s), m(t)\rangle \in R_{A}$; from the former, by $\mathrm{TB}(>)$, any $w \in W$ such that $m(s) R_{A} w$ has $v_{w}(B)=1$; so $v_{m(t)}(B)=1$. This is impossible; reject the assumption: $\Gamma_{k} \models_{B x}^{*} B_{t}$, which is to say, $\Gamma_{k} \models_{B x}^{*} \mathcal{P}_{k}$.

$\left(A M P_{I}\right)$ If $\mathcal{P}_{k}$ arises by $A M P_{I}$, then the picture is like this,

$$
\begin{array}{l|l}
i & A_{0 / t} \\
k & A_{t}
\end{array}
$$

where $i<k$ and $\mathcal{P}_{k}$ is $A_{t}$. Where this rule is in $N B x, B x$ includes condition (I). By assumption, $\Gamma_{\mathrm{i}} \models_{\mathrm{Bx}}^{*} A_{\mathrm{O} / \mathrm{t}}$; but by the nature of access, $\Gamma_{i} \subseteq \Gamma_{k}$; so by L8.I, $\Gamma_{k} \models_{B x}^{*} A_{0 / t}$. Suppose $\Gamma_{k} \not \nvdash_{B x}^{*} A_{t}$; then by VBx*, there is some $B x$ interpretation with $\mathrm{N},\left\{R_{A} \mid A \in \mathfrak{I}\right\}, v$ and $m$ such that $v_{m}\left(\Gamma_{k}\right)=1$ but $v_{m(t)}(A)=0$; since $v_{m}\left(\Gamma_{k}\right)=1$, by $V_{B x}^{*}, m(t) \in$ $f_{A}(m(0))$; but by the construction of $m, m(0) \in N$; so by condition $(I)$, 
$m(t) \in[A]$; so $v_{m(t)}(A)=1$. This is impossible; reject the assumption: $\Gamma_{k} \models_{B x}^{*} A_{t}$, which is to say, $\Gamma_{k} \models_{B x}^{*} \mathcal{P}_{k}$.

$\left(\mathrm{AMP}_{2}\right)$ If $\mathcal{P}_{k}$ arises by $\mathrm{AMP}_{2}$, then the picture is like this,

$$
\begin{array}{l|l}
i & A_{0} \\
k & A_{0 / 0}
\end{array}
$$

where $i<k$ and $\mathcal{P}_{k}$ is $A_{0 / 0}$. Where this rule is in $N B x, B x$ includes condition (2). By assumption, $\Gamma_{\mathrm{i}} \models_{B x}^{*} A_{0}$; but by the nature of access, $\Gamma_{i} \subseteq \Gamma_{k}$; so by L8.I, $\Gamma_{k} \models_{B x}^{*} A_{0}$. Suppose $\Gamma_{k} \forall_{B x}^{*} A_{0 / 0}$; then by VBx*, there is some $B x$ interpretation with $\mathrm{N},\left\{\mathrm{R}_{\mathrm{A}} \mid A \in \mathfrak{I}\right\}, v$ and $\mathrm{m}$ such that $v_{\mathrm{m}}\left(\Gamma_{\mathrm{k}}\right)=1$ but $\mathrm{m}(0) \notin \mathrm{f}_{\mathrm{A}}(\mathrm{m}(0))$; since $v_{\mathrm{m}}\left(\Gamma_{\mathrm{k}}\right)=1$, by $\mathrm{VCx}^{*}$, $v_{m(0)}(A)=1$; so $m(0) \in[A]$; and by the construction of $m, m(0) \in N$; so by condition (2), $m(0) \in f_{A}(m(0))$. This is impossible; reject the assumption: $\Gamma_{k} \models_{B x}^{*} A_{0 / 0}$, which is to say, $\Gamma_{k} \models_{B x}^{*} \mathcal{P}_{k}$.

For any $i, \Gamma_{i} \models_{B x}^{*} \mathcal{P}_{i}$.

THEOREM 8.2 NBx is complete: if $\Gamma \models_{\mathrm{B} x}$ A then $\Gamma \vdash_{\mathrm{NBx}} \mathrm{A}$.

Suppose $\Gamma \models_{B x} A$; then $\Gamma_{0} \models_{B x}^{*} A_{0}$; we show that $\Gamma_{0} \vdash_{N B x}^{*} A_{0}$. As usual, this reduces to the standard notion. For the following, fix on some particular constraint(s) $x$. Then definitions of consistency etc. are relative to it.

Con $\Gamma$ is CONSISTENT iff there is no $A_{s}$ such that $\Gamma \vdash_{\mathrm{NBx}}^{*} A_{s}$ and $\Gamma \vdash_{\mathrm{NBx}}^{*} \neg A_{\bar{s}}$.

L8.2 If $s$ is 0 or introduced in $\Gamma$, and $\Gamma \nvdash_{\mathrm{NB} x}^{*} \neg \mathrm{P}_{\overline{\mathrm{s}}}$, then $\Gamma \cup\left\{\mathrm{P}_{\mathrm{s}}\right\}$ is consistent.

Suppose $s$ is 0 or introduced in $\Gamma$ and $\Gamma \nvdash_{N B x}^{*} \neg P_{\bar{s}}$ but $\Gamma \cup\left\{P_{s}\right\}$ is inconsistent. Then there is some $A_{t}$ such that $\Gamma \cup\left\{P_{s}\right\} \vdash_{\mathrm{NBx}}^{*} A_{t}$ and $\Gamma \cup\left\{\mathrm{P}_{\mathrm{s}}\right\} \vdash_{\mathrm{NBx}}^{*} \neg \mathrm{A}_{\overline{\mathrm{t}}}$. But then we can argue,



where the assumption is allowed insofar as $s$ is either 0 or introduced in $\Gamma$; so $\Gamma \vdash_{\mathrm{NBx}}^{*} \neg \mathrm{P}_{\overline{\mathrm{s}}}$. But this is impossible; reject the assumption: if $s$ is 0 or introduced in $\Gamma$ and $\Gamma H_{\mathrm{NBx}}^{*} \neg \mathrm{P}_{\bar{s}}$, then $\Gamma \cup\left\{\mathrm{P}_{\mathrm{s}}\right\}$ is consistent.

L8.3 There is an enumeration of all the subscripted formulas, $\mathcal{P}_{1} \mathcal{P}_{2} \ldots$ In addition, there is an enumeration of these formulas with access relations s.t.u and with pairs of the sort s.t.u / u.v.w.

Proof by construction. 
Max $\Gamma$ is s-maXimal iff for any $A_{s}$ either $\Gamma \vdash_{\mathrm{NBx}}^{*} A_{s}$ or $\Gamma \vdash_{\mathrm{NBx}}^{*} \neg A_{\overline{\mathrm{s}}}$.

SGT $\Gamma$ is a SCAPEGOAT set for $\rightarrow$ iff for every formula of the form $\neg(A \rightarrow B)_{s}$, if $\Gamma \vdash_{\mathrm{NBx}}^{*} \neg(A \rightarrow B)_{s}$ then there are $y$ and $z$ such that $\Gamma \vdash_{\mathrm{NBx}}^{*} \bar{s} . y . z$, $\Gamma \vdash_{\mathrm{NB}}^{*} A_{\mathrm{y}}$ and $\Gamma \vdash_{\mathrm{NBx}}^{*} \neg \mathrm{B}_{\bar{z}}$.

$\Gamma$ is a SCAPEGOAT set for $>$ iff for every formula of the form $\neg(A>B)_{s}$, if $\Gamma \vdash_{\mathrm{NBx}}^{*} \neg(A>B)_{s}$ then there is some $y$ such that $\Gamma \vdash_{\mathrm{NBx}}^{*} A_{\bar{s} / y}$ and $\Gamma \vdash_{\mathrm{NBx}}^{*} \neg \mathrm{B}_{\bar{y}}$.

$\Gamma$ is a SCAPEgOAT set for C9/Cio iff for any access pair s.t.u / u.v.w, if $\Gamma \vdash_{\mathrm{NBx}}^{*}$ s.t.u and $\Gamma \vdash_{\mathrm{NBx}}^{*}$ u.v.w, then there is a $y$ such that $\Gamma \vdash_{\mathrm{NBx}}^{*}$ s.v.y and $\Gamma \vdash_{\mathrm{NB} x}^{*}$ t.y.w, and a $z$ such that $\Gamma \vdash_{\mathrm{NBx}}^{*}$ t.v.z and $\Gamma \vdash_{\mathrm{NBx}}^{*}$ s.z.w.

$\Gamma$ is a SCAPEGOAT set for CI2 iff for any access relation s.t.u, if $\Gamma \vdash_{\mathrm{NBx}}^{*}$ s.t.u, then there is a $y$ such that $\Gamma \vdash_{\mathrm{NBx}}^{*}$ s.t.y and $\Gamma \vdash_{\mathrm{NBx}}^{*}$ y.t.u.

$\mathrm{C}\left(\Gamma^{\prime}\right)$ For $\Gamma$ with unsubscripted formulas and the corresponding $\Gamma_{0}$, we construct $\Gamma^{\prime}$ as follows. Set $\Omega_{0}=\Gamma_{0}$. By L8.3, there is an enumeration, $\mathcal{P}_{1}, \mathcal{P}_{2} \ldots$ of all the subscripted formulas, together with all the access relations s.t.u if $\mathrm{C}_{2} 2$ is in $B x$, and pairs s.t.u / u.v.w if $\mathrm{C}_{9}$ and $\mathrm{Cro}_{1}$ are in $B x$; let $\mathcal{E}_{0}$ be this enumeration. Then for the first expression $\mathcal{P}$ in $\mathcal{E}_{i-1}$ such that all its subscripts are o or introduced in $\Omega_{i-1}$, let $\mathcal{E}_{i}$ be like $\mathcal{E}_{i-1}$ but without $\mathcal{P}$, and set,

$$
\begin{aligned}
& \Omega_{i}=\Omega_{i-1} \quad \text { if } \Omega_{i-1} \cup\{\mathcal{P}\} \text { is inconsistent } \\
& \Omega_{i^{*}}=\Omega_{i-1} \cup\{\mathcal{P}\} \quad \text { if } \Omega_{i-1} \cup\{\mathcal{P}\} \text { is consistent } \\
& \text { and } \\
& \Omega_{i}=\Omega_{i^{*}} \quad \text { if } \mathcal{P} \text { is not of the form } \neg(\mathrm{P} \rightarrow \\
& \mathrm{Q})_{s}, \neg(\mathrm{P}>\mathrm{Q})_{s} \text {, s.t.u / u.v.w, } \\
& \text { or s.t.u } \\
& \Omega_{\mathrm{i}}=\Omega_{\mathrm{i}^{*}} \cup\left\{\bar{s} . y . z, \mathrm{P}_{\mathrm{y}} \neg \mathrm{Q}_{\bar{z}}\right\} \quad \text { if } \mathcal{P} \text { is of the form } \neg(\mathrm{P} \rightarrow \mathrm{Q})_{s} \\
& \Omega_{\mathrm{i}}=\Omega_{\mathrm{i}^{*}} \cup\left\{\mathrm{P}_{\overline{\mathrm{s}} / \mathrm{y}}, \neg \mathrm{Q}_{\bar{y}}\right\} \quad \text { if } \mathcal{P} \text { is of the form } \neg(\mathrm{P}>\mathrm{Q})_{s} \\
& \Omega_{i}=\Omega_{i^{*}} \cup\{\text { s.v.y, t.y.w, t.v.z, s.z.w }\} \text { if } \mathcal{P} \text { is of the form s.t.u / u.v.w } \\
& \Omega_{\mathfrak{i}}=\Omega_{\mathfrak{i}^{*}} \cup\{\text { s.t.y, y.t.u }\} \quad \text { if } \mathcal{P} \text { is of the form s.t.u } \\
& \text {-where } y \text { and } z \text { are the first subscripts not introduced in } \Omega_{\mathfrak{i}^{*}} \\
& \text { then } \\
& \Gamma^{\prime}=\bigcup_{i \geqslant 0} \Omega_{i}
\end{aligned}
$$

Note that there are always sure to be subscripts $y$ and $z$ not in $\Omega_{\mathfrak{i}^{*}}$ insofar as there are infinitely many subscripts, and at any stage only finitely many expressions are added - the only subscripts in the initial $\Omega_{0}$ being 0 . Suppose $s$ is introduced in $\Gamma^{\prime}$; then there is some $\Omega_{i}$ in which it is first introduced; and any expression $\mathcal{P}_{j}$ in the original enumeration that introduces subscript $s$ is sure to be "considered" for inclusion at a subsequent stage.

L8.4 For any s introduced in $\Gamma^{\prime}, \Gamma^{\prime}$ is s-maximal. 
Suppose $s$ is introduced in $\Gamma^{\prime}$ but $\Gamma^{\prime}$ is not s-maximal. Then there is some $A_{s}$ such that $\Gamma^{\prime} \nvdash_{\mathrm{NBx}}^{*} A_{s}$ and $\Gamma^{\prime} \nvdash_{\mathrm{NBx}}^{*} \neg A_{\bar{s}}$. For any $i$, each member of $\Omega_{i-1}$ is in $\Gamma^{\prime}$; so if $\Omega_{i-1} \vdash_{\mathrm{NBx}}^{*} \neg A_{\bar{s}}$ then $\Gamma^{\prime} \vdash_{\mathrm{NBx}}^{*} \neg A_{\bar{s}}$; but $\Gamma^{\prime} \nvdash_{\mathrm{NBx}}^{*} \neg A_{\bar{s}}$; so $\Omega_{i-1} \nvdash_{\mathrm{NBx}}^{*} \neg A_{\bar{s}}$; so since $s$ is introduced in $\Gamma^{\prime}$, by L8.2, $\Gamma^{\prime} \cup\left\{A_{s}\right\}$ is consistent; so there is a stage in the construction that sets $\Omega_{i^{*}}=\Omega_{i-1} \cup$ $\left\{A_{s}\right\}$; so by construction, $A_{s} \in \Gamma^{\prime}$; so $\Gamma^{\prime} \vdash_{\mathrm{NB} x}^{*} A_{s}$. This is impossible; reject the assumption: $\Gamma^{\prime}$ is s-maximal.

L8.5 If $\Gamma_{0}$ is consistent, then each $\Omega_{i}$ is consistent.

Suppose $\Gamma_{0}$ is consistent.

Basis: $\Omega_{0}=\Gamma_{0}$ and $\Gamma_{0}$ is consistent; so $\Omega_{0}$ is consistent.

Assp: For any $i, 0 \leqslant i<k, \Omega_{i}$ is consistent.

Show: $\Omega_{\mathrm{k}}$ is consistent.

We know that $\Omega_{k}$ is either (i) $\Omega_{k-1}$, (ii) $\Omega_{k^{*}}=\Omega_{k-1} \cup\{\mathcal{P}\}$, (iii) $\Omega_{k^{*}} \cup\left\{\bar{s} . y . z, P_{y}, \neg Q_{\bar{z}}\right\}$, (iv) $\Omega_{k^{*}} \cup\left\{P_{\bar{s} / y}, \neg Q_{\bar{y}}\right\}$, (v) $\Omega_{k^{*}} \cup\{$ s.v.y, t.y.w, t.v.z, s.z.w $\}$, or (vi) $\Omega_{k^{*}} \cup\{$ s.t.y, y.t.u\}.

(i) Suppose $\Omega_{k}$ is $\Omega_{k-1}$. By assumption, $\Omega_{k-1}$ is consistent; so $\Omega_{k}$ is consistent.

(ii) Suppose $\Omega_{k}$ is $\Omega_{k^{*}}=\Omega_{k-1} \cup\{\mathcal{P}\}$. Then by construction, $\Omega_{k-1} \cup$ $\{\mathcal{P}\}$ is consistent; so $\Omega_{k}$ is consistent.

(iii) Suppose $\Omega_{\mathrm{k}}$ is $\Omega_{\mathrm{k}^{*}} \cup\left\{\bar{s}\right.$.y.z, $\left.\mathrm{P}_{\mathrm{y}}, \neg \mathrm{Q}_{\bar{z}}\right\}$. In this case, as above, $\Omega_{\mathrm{k} *}$ is consistent and by construction, $\neg(\mathrm{P} \rightarrow \mathrm{Q})_{\mathrm{s}} \in \Omega_{\mathrm{k}^{*}}$. Suppose $\Omega_{\mathrm{k}}$ is inconsistent. Then there are $A_{x}$ and $\neg A_{\bar{x}}$ such that $\Omega_{\mathrm{k}^{*}} \cup$ $\left\{\bar{s} . y . z, \mathrm{P}_{\mathrm{y}}, \neg \mathrm{Q}_{\bar{z}}\right\} \vdash_{\mathrm{NBx}}^{*} A_{x}$ and $\Omega_{\mathrm{k}^{*}} \cup\left\{\bar{s} . y . z, \mathrm{P}_{\mathrm{y}}, \neg \mathrm{Q}_{\bar{z}}\right\} \vdash_{\mathrm{NBx}}^{*} \neg A_{\bar{x}}$. So reason as follows,

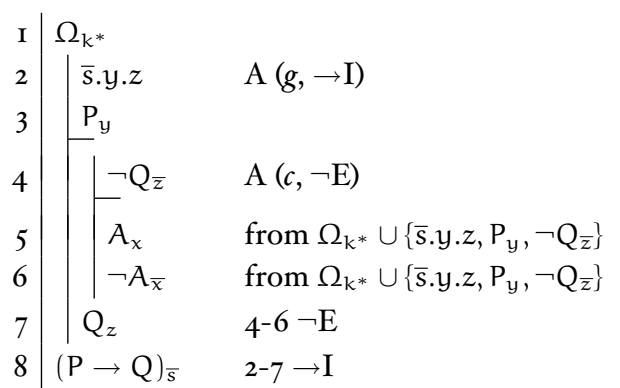

where, by construction, $y$ and $z$ are not introduced $\Omega_{\mathrm{k}^{*}}$. So $\Omega_{\mathrm{K}^{*}} \vdash_{\mathrm{NBx}}^{*}(\mathrm{P} \rightarrow \mathrm{Q})_{\bar{s}}$; but $\neg(\mathrm{P} \rightarrow \mathrm{Q})_{s} \in \Omega_{\mathrm{k}^{*}} ;$ so $\Omega_{\mathrm{k}^{*}} \vdash_{\mathrm{NBx}}^{*} \neg(\mathrm{P} \rightarrow$ Q) $)_{s}$; so $\Omega_{\mathrm{k}^{*}}$ is inconsistent. This is impossible; reject the assumption: $\Omega_{k}$ is consistent.

(iv) Suppose $\Omega_{k}$ is $\Omega_{k^{*}} \cup\left\{P_{\bar{s} / y}, \neg Q_{\bar{y}}\right\}$. In this case, as above, $\Omega_{k *}$ is consistent and by construction, $\neg(\mathrm{P}>\mathrm{Q})_{\mathrm{s}} \in \Omega_{\mathrm{k}^{*}}$. Suppose $\Omega_{\mathrm{k}}$ is inconsistent. Then there are $A_{\mathrm{x}}$ and $\neg A_{\overline{\mathrm{x}}}$ such that $\Omega_{\mathrm{k}^{*}} \cup$ 
$\left\{\mathrm{P}_{\overline{\mathrm{s}} / \mathrm{y}}, \neg \mathrm{Q}_{\bar{y}}\right\} \vdash_{\mathrm{NB} x}^{*} A_{x}$ and $\Omega_{\mathrm{k}^{*}} \cup\left\{\mathrm{P}_{\overline{\mathrm{s}} / \mathrm{y}}, \neg \mathrm{Q}_{\bar{y}}\right\} \vdash_{\mathrm{NB} x}^{*} \neg A_{\overline{\mathrm{x}}}$. So reason as follows,

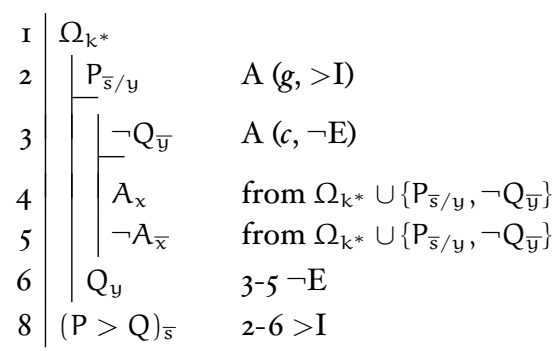

where, by construction, $y$ is not introduced $\Omega_{\mathrm{k}^{*}}$. So $\Omega_{\mathrm{k}^{*}} \vdash_{\mathrm{NBx}}^{*}$ $(\mathrm{P}>\mathrm{Q})_{\bar{s}}$; but $\neg(\mathrm{P}>\mathrm{Q})_{\mathrm{s}} \in \Omega_{\mathrm{K}^{*}}$; so $\Omega_{\mathrm{K}^{*}} \vdash_{\mathrm{NBx}}^{*} \neg(\mathrm{P}>\mathrm{Q})_{\mathrm{s}}$; so $\Omega_{\mathrm{K}^{*}}$ is inconsistent. This is impossible; reject the assumption: $\Omega_{k}$ is consistent.

(v) Suppose $\Omega_{k}$ is $\Omega_{k^{*}} \cup\{$ s.v.y, t.y.w, t.v.z, s.z.w $\}$. In this case, as above, $\Omega_{\mathrm{k} *}$ is consistent and by construction, s.t.u, u.v.w $\in \Omega_{\mathrm{k}^{*}}$. Suppose $\Omega_{k}$ is inconsistent. Then there are $A_{x}$ and $\neg A_{\bar{x}}$ such that $\Omega_{\mathrm{k}^{*}} \cup\{$ s.v.y, t.y.w, t.v.z, s.z.w $\} \vdash_{\mathrm{NBx}}^{*} A_{\mathrm{x}}$ and in addition, $\Omega_{\mathrm{k}^{*}} \cup$ $\{$ s.v.y, t.y.w, t.v.z, s.z.w $\} \vdash_{\mathrm{NBx}}^{*} \neg A_{\overline{\mathrm{x}}}$. So reason as follows,

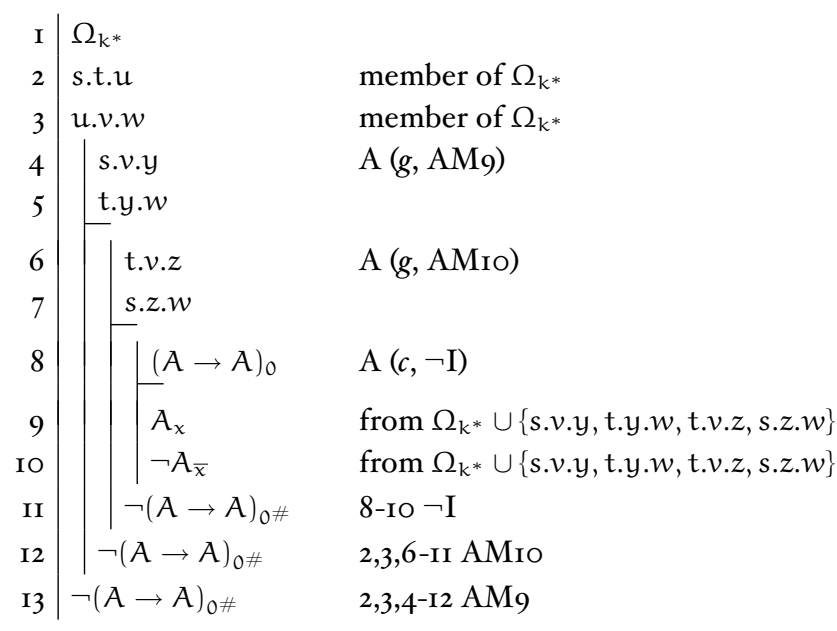

where, by construction, $y$ and $z$ are not introduced $\Omega_{k^{*}}$. So $\Omega_{\mathrm{k}^{*}} \vdash_{\mathrm{NBx}}^{*} \neg(A \rightarrow A)_{\mathrm{O}^{\#}}$; but $\vdash_{\mathrm{NBx}}^{*}(A \rightarrow A)_{0}$; so $\Omega_{\mathrm{k}^{*}}$ is inconsistent. This is impossible; reject the assumption: $\Omega_{k}$ is consistent.

(vi) Similarly.

For any $i, \Omega_{i}$ is consistent.

L8.6 If $\Gamma_{0}$ is consistent, then $\Gamma^{\prime}$ is consistent.

Suppose $\Gamma_{0}$ is consistent, but $\Gamma^{\prime}$ is not; from the latter, there is some $\mathrm{P}_{\mathrm{s}}$ such that $\Gamma^{\prime} \vdash_{\mathrm{NBx}}^{*} \mathrm{P}_{\mathrm{S}}$ and $\Gamma^{\prime} \vdash_{\mathrm{NBx}}^{*} \neg \mathrm{P}_{\overline{\mathrm{s}}}$. Consider derivations Di and D2 of these results, and the premises $\mathcal{P}_{i} \ldots \mathcal{P}_{j}$ of these derivations. By 
construction, there is an $\Omega_{k}$ with each of these premises as a member; so Dr and D2 are derivations from $\Omega_{k}$; so $\Omega_{k}$ is not consistent. But since $\Gamma_{0}$ is consistent, by L8.5, $\Omega_{k}$ is consistent. This is impossible; reject the assumption: if $\Gamma_{0}$ is consistent then $\Gamma^{\prime}$ is consistent.

L8.7 If $\Gamma_{0}$ is consistent, then $\Gamma^{\prime}$ is a scapegoat set for $\rightarrow,>$ and, in the appropriate systems, for $\mathrm{C}_{9} / \mathrm{Cr}_{\mathrm{O}}$ and $\mathrm{Ci}_{2}$.

For $\rightarrow$. Suppose $\Gamma_{0}$ is consistent and $\Gamma^{\prime} \vdash_{\mathrm{NBx}}^{*} \neg(\mathrm{P} \rightarrow \mathrm{Q})_{s}$. By L8.6, $\Gamma^{\prime}$ is consistent; and by the constraints on subscripts, $s$ is introduced in $\Gamma^{\prime}$. Since $\Gamma^{\prime} \vdash_{\mathrm{NBx}}^{*} \neg(\mathrm{P} \rightarrow \mathrm{Q})_{s}, \Gamma^{\prime}$ has just the same consequences as $\Gamma^{\prime} \cup\left\{\neg(\mathrm{P} \rightarrow \mathrm{Q})_{s}\right\} ;$ so $\Gamma^{\prime} \cup\left\{\neg(\mathrm{P} \rightarrow \mathrm{Q})_{s}\right\}$ is consistent, and for any $\Omega_{j}, \Omega_{j} \cup$ $\left\{\neg(P \rightarrow Q)_{s}\right\}$ is consistent. So there is a stage in the construction process where $\Omega_{i^{*}}=\Omega_{i-1} \cup\{\neg(P \rightarrow Q) s\}$ and $\Omega_{i}=\Omega_{i^{*}} \cup\left\{\bar{s} . y . z, P_{y}, \neg Q_{\bar{z}}\right\}$; so by construction, s.y.z, $\mathrm{P}_{y}, \neg \mathrm{Q}_{\bar{z}} \in \Gamma^{\prime}$; so $\Gamma^{\prime} \vdash_{\mathrm{NBx}}^{*} \bar{s} . y . z, \Gamma^{\prime} \vdash_{\mathrm{NBx}}^{*} \mathrm{P}_{y}$ and $\Gamma^{\prime} \vdash_{\mathrm{NBx}}^{*} \neg \mathrm{Q}_{\bar{z}}$. So $\Gamma^{\prime}$ is a scapegoat set for $\rightarrow$.

For $>$. Suppose $\Gamma_{0}$ is consistent and $\Gamma^{\prime} \vdash_{\mathrm{NBx}}^{*} \neg(\mathrm{P}>\mathrm{Q})_{s}$. By L8.6, $\Gamma^{\prime}$ is consistent; and by the constraints on subscripts, $s$ is introduced in $\Gamma^{\prime}$. Since $\Gamma^{\prime} \vdash_{\mathrm{NBx}}^{*} \neg(\mathrm{P}>\mathrm{Q})_{s}, \Gamma^{\prime}$ has just the same consequences as $\Gamma^{\prime} \cup$ $\left\{\neg(\mathrm{P}>\mathrm{Q})_{s}\right\}$; so $\Gamma^{\prime} \cup\left\{\neg(\mathrm{P}>\mathrm{Q})_{s}\right\}$ is consistent, and for any $\Omega_{j}, \Omega_{j} \cup$ $\left\{\neg(\mathrm{P}>\mathrm{Q})_{s}\right\}$ is consistent. So there is a stage in the construction process where $\Omega_{i^{*}}=\Omega_{i-1} \cup\left\{\neg(P>Q)_{s}\right\}$ and $\Omega_{i}=\Omega_{i^{*}} \cup\left\{P_{\bar{s} / y}, \neg Q_{\bar{y}}\right\}$; so by construction, $\mathrm{P}_{\overline{\mathrm{s}} / \mathrm{y}}, \neg \mathrm{Q}_{\overline{\mathrm{y}}} \in \Gamma^{\prime}$; so $\Gamma^{\prime} \vdash_{\mathrm{NBx}}^{*} \mathrm{P}_{\overline{\mathrm{s}} / \mathrm{y}}$ and $\Gamma^{\prime} \vdash_{\mathrm{NBx}}^{*} \neg \mathrm{Q}_{\overline{\mathrm{y}}}$. So $\Gamma^{\prime}$ is a scapegoat set for $>$.

For C9/Cio. Suppose $\Gamma_{0}$ is consistent, $\Gamma^{\prime} \vdash_{\mathrm{NBx}}^{*}$ s.t.u and $\Gamma^{\prime} \vdash_{\mathrm{NBx}}^{*}$ u.v.w. By L8.6, $\Gamma^{\prime}$ is consistent; and by the constraints on subscripts, s, t, $u, v$ and $w$ are introduced in $\Gamma^{\prime}$. Since $\Gamma^{\prime} \vdash_{\mathrm{NBx}}^{*}$ s.t.u, and $\Gamma^{\prime} \vdash_{\mathrm{NBx}}^{*} u . v . w, \Gamma^{\prime}$ has just the same consequences as $\Gamma^{\prime} \cup\{$ s.t.u, u.v.w $\}$; so $\Gamma^{\prime} \cup\{$ s.t.u, u.v.w $\}$ is consistent, and for any $\Omega_{j}, \Omega_{j} \cup\{$ s.t.u, u.v.w $\}$ is consistent. So there is a stage in the construction process where $\Omega_{\mathfrak{i}^{*}}=\Omega_{\mathfrak{i}-1} \cup\{$ s.t.u, u.v.w $\}$ and $\Omega_{\mathfrak{i}}=\Omega_{i^{*}} \cup\{$ s.v.y, t.y.w, t.v.z, s.z.w $\}$; so by construction, s.v.y, t.y.w, t.v.z, s.z.w $\in \Gamma^{\prime}$; so there is a $y$ such that $\Gamma^{\prime} \vdash_{\mathrm{NBx}}^{*}$ s.v.y and $\Gamma^{\prime} \vdash_{\mathrm{NBx}}^{*}$ t.y.w, and there is a $z$ such that $\Gamma^{\prime} \vdash_{\mathrm{NBx}}^{*}$ t.v. $z$ and $\Gamma^{\prime} \vdash_{\mathrm{NBx}}^{*}$ s.z.w. So $\Gamma^{\prime}$ is a scapegoat set for $\mathrm{C}_{9} / \mathrm{Cr}_{\mathrm{o}}$. And similarly for Ci2.

$\mathrm{C}(\mathrm{I})$ We construct an interpretation $\mathrm{I}_{\mathrm{Bx}}=\langle\mathrm{W}, \mathrm{N}, \mathrm{R}, *, v\rangle$ or $\left\langle\mathrm{W}, \mathrm{N}, \mathrm{R},\left\{\mathrm{R}_{\mathrm{A}} \mid\right.\right.$ $\mathrm{A} \in \mathfrak{I}\}, *, v\rangle$ based on $\Gamma^{\prime}$ as follows. Let $\mathrm{W}$ have a member $w_{\mathrm{s}}$ corresponding to each subscript $s$ introduced in $\Gamma^{\prime}$, except that if $\Gamma^{\prime} \vdash_{\mathrm{NBx}}^{*} \mathrm{~s} \simeq \mathrm{t}$ then $w_{s}=w_{t}$ and $w_{\bar{s}}=w_{\bar{t}}$ (we might do this, in the usual way, by beginning with equivalence classes on subscripts). Then set $N=\left\{w_{0}\right\}$; $\left\langle w_{s}, w_{t}, w_{u}\right\rangle \in R$ iff $\Gamma^{\prime} \vdash_{\mathrm{NBx}}^{*}$ s.t.u; $\left\langle w_{s}, w_{t}\right\rangle \in R_{A}$ iff $\Gamma^{\prime} \vdash_{\mathrm{NBx}}^{*} A_{s / t} ;$ $*=\left\{\left\langle w_{s}, w_{\bar{s}}\right\rangle \mid s\right.$ is introduced in $\left.\Gamma^{\prime}\right\}$; and $v_{w_{s}}(p)=1$ iff $\Gamma^{\prime} \vdash_{\mathrm{NBx}}^{*} p_{s}$.

Note that the specification is consistent: Suppose $w_{\mathrm{s}}=w_{\mathrm{t}}$; then by construction, $\Gamma^{\prime} \vdash_{\mathrm{NBx}}^{*} \mathrm{~s} \simeq \mathrm{t}$; so by $\simeq \mathrm{E}, \Gamma^{\prime} \vdash_{\mathrm{NBx}}^{*} p_{\mathrm{s}}$ iff $\Gamma^{\prime} \vdash_{\mathrm{NBx}}^{*} p_{\mathrm{t}} ;$ so $v_{w_{\mathrm{s}}}(\mathrm{p})=$ 
$v_{w_{\mathrm{t}}}(\mathrm{p})$; and similarly in other cases. Also, the $*$-function has the right form, as $s, \bar{s}$ are introduced in pairs, and $\left\langle w_{s}, w_{s \#}\right\rangle \in *$ iff $\left\langle w_{s^{*}}, w_{s}\right\rangle \in *$.

L8.8 If $\Gamma_{0}$ is consistent then for $\mathrm{I}_{\mathrm{Bx}}$ constructed as above, and for any $\mathrm{s}$ introduced in $\Gamma^{\prime}, v_{w_{s}}(A)=1$ iff $\Gamma^{\prime} \vdash_{\mathrm{NB} x}^{*} A_{s}$.

Suppose $\Gamma_{0}$ is consistent and $s$ is introduced in $\Gamma^{\prime}$. By L8.4, $\Gamma^{\prime}$ is smaximal. By L8.6 and L8.7, $\Gamma^{\prime}$ is consistent and a scapegoat set for $\rightarrow$ and $>$. Now by induction on the number of operators in $A_{s}$,

Basis: If $A_{s}$ has no operators, then it is a parameter $p_{s}$ and by construction, $v_{w_{s}}(p)=1$ iff $\Gamma^{\prime} \vdash_{\mathrm{NBx}}^{*} p_{s}$. So $v_{w_{s}}(A)=1$ iff $\Gamma^{\prime} \vdash_{\mathrm{NBx}}^{*} A_{s}$.

Assp: For any $i, 0 \leqslant i<k$, if $A_{s}$ has $i$ operators, then $v_{w_{s}}(A)=1$ iff $\Gamma^{\prime} \vdash_{\mathrm{NBx}}^{*} A_{s}$.

Show: If $A_{s}$ has $k$ operators, then $v_{w_{s}}(A)=1$ iff $\Gamma^{\prime} \vdash_{\mathrm{NBx}}^{*} A_{s}$.

If $A_{s}$ has $k$ operators, then it is of the form $\neg P_{s},(P \wedge Q)_{s},(P \vee$ $\mathrm{Q})_{s},(\mathrm{P} \rightarrow \mathrm{Q})_{s}$, or $(\mathrm{P}>\mathrm{Q})_{s}$ where $\mathrm{P}$ and $\mathrm{Q}$ have $<\mathrm{k}$ operators.

$(\neg) A_{s}$ is $\neg P_{s}$. (i) Suppose $v_{w_{s}}(A)=1$; then $v_{w_{s}}(\neg P)=1$; so by $\mathrm{TB}(\neg), v_{w_{s}^{*}}(\mathrm{P})=0$; so by construction, $v_{w_{\bar{s}}}(\mathrm{P})=0$; so by assumption, $\Gamma^{\prime} \nvdash_{\mathrm{NBx}}^{*} \mathrm{P}_{\bar{s}}$; so by s-maximality, $\Gamma^{\prime} \vdash_{\mathrm{NBx}}^{*} \neg \mathrm{P}_{s}$, where this is to say, $\Gamma^{\prime} \vdash_{\mathrm{NBx}}^{*} A_{s}$. (ii) Suppose $\Gamma^{\prime} \vdash_{\mathrm{NBx}}^{*} A_{s}$; then $\Gamma^{\prime} \vdash_{\mathrm{NBx}}^{*} \neg P_{s}$; so by consistency, $\Gamma^{\prime} \nvdash_{\mathrm{NBx}}^{*} \mathrm{P}_{\overline{\mathrm{s}}}$; so by assumption, $v_{w_{\bar{s}}}(\mathrm{P})=0$; so by construction, $v_{w_{s}^{*}}(\mathrm{P})=0$; so by $\mathrm{TB}(\neg), v_{w_{s}}(\neg \mathrm{P})=1$, where this is to say, $v_{w_{s}}(A)=1$. So $v_{w_{s}}(A)=1 \mathrm{iff} \Gamma^{\prime} \vdash_{\mathrm{NBx}}^{*} A_{s}$.

$(\wedge)$

$(\mathrm{V})$

$(\rightarrow) A_{s}$ is $(P \rightarrow Q)_{s}$. (i) Suppose $v_{w_{s}}(A)=1$ but $\Gamma^{\prime} \nvdash_{N B x}^{*} A_{s}$; then $v_{w_{s}}(\mathrm{P} \rightarrow \mathrm{Q})=1$ but $\Gamma^{\prime} \forall_{\mathrm{NBx}}^{*}(\mathrm{P} \rightarrow \mathrm{Q})_{s}$. From the latter, by $s-$ maximality, $\Gamma^{\prime} \vdash_{\mathrm{NBx}}^{*} \neg(\mathrm{P} \rightarrow \mathrm{Q})_{\bar{s}}$; so, since $\Gamma^{\prime}$ is a scapegoat set for $\rightarrow$, there are some $y$ and $z$ such that $\Gamma^{\prime} \vdash_{\mathrm{NBx}}^{*}$ s.y.z, $\Gamma^{\prime} \vdash_{\mathrm{NBx}}^{*} \mathrm{P}_{\mathrm{y}}$ and $\Gamma^{\prime} \vdash_{\mathrm{NBx}}^{*} \neg \mathrm{Q}_{\bar{z}}$; from the latter, by consistency, $\Gamma^{\prime}{\nvdash_{\mathrm{NBx}}^{*}}^{*} \mathrm{Q}_{z}$; so by assumption, $v_{w_{y}}(\mathrm{P})=1$ and $v_{w_{z}}(\mathrm{Q})=0$; but since $\Gamma^{\prime} \vdash_{\mathrm{NBx}}^{*}$ s.y.z, by construction, $\left\langle w_{s}, w_{y}, w_{z}\right\rangle \in \mathrm{R}$; so by $\mathrm{TB}(\rightarrow), v_{w_{s}}(\mathrm{P} \rightarrow \mathrm{Q})=$ 0 . This is impossible; reject the assumption: if $v_{w_{s}}(A)=1$ then $\Gamma^{\prime} \vdash_{\mathrm{NBx}}^{*} A_{s}$.

(ii) Suppose $\Gamma^{\prime} \vdash_{\mathrm{NBx}}^{*} A_{\mathrm{s}}$ but $v_{w_{\mathrm{s}}}(\mathrm{A})=0$; then $\Gamma^{\prime} \vdash_{\mathrm{NBx}}^{*}(\mathrm{P} \rightarrow \mathrm{Q})_{\mathrm{s}}$ but $v_{w_{s}}(\mathrm{P} \rightarrow \mathrm{Q})=0$. From the latter, by $\mathrm{TB}(\rightarrow)$, there are some $w_{\mathrm{t}}, w_{\mathrm{u}} \in \mathrm{W}$ such that $\left\langle w_{\mathrm{s}}, w_{\mathrm{t}}, w_{\mathrm{u}}\right\rangle \in \mathrm{R}$ and $v_{w_{\mathrm{t}}}(\mathrm{P})=1$ but $v_{w_{u}}(\mathrm{Q})=0$; so by assumption, $\Gamma^{\prime} \vdash_{\mathrm{NBx}}^{*} P_{\mathrm{t}}$ and $\Gamma^{\prime} \nvdash_{\mathrm{NBx}}^{*} \mathrm{Q}_{u}$; so by s-maximality, $\Gamma^{\prime} \vdash_{\mathrm{NBx}}^{*} \neg \mathrm{Q}_{\bar{u}}$. Since $\left\langle w_{s}, w_{\mathrm{t}}, w_{\mathrm{u}}\right\rangle \in R$, by construction, $\Gamma^{\prime} \vdash_{\mathrm{NBx}}^{*}$ s.t.u; so by reasoning as follows, 


\begin{tabular}{|c|c|c|}
\hline & $\Gamma^{\prime}$ & \\
\hline & $(\mathrm{P} \rightarrow \mathrm{Q})_{s}$ & $\mathrm{~A}(c, \neg \mathrm{I})$ \\
\hline & s.t.u & from $\Gamma^{\prime}$ \\
\hline & $P_{t}$ & from $\Gamma^{\prime}$ \\
\hline & $\mathrm{Q}_{\mathrm{u}}$ & $3,2,4 \rightarrow \mathrm{E}$ \\
\hline & $\neg \mathrm{Q}_{\overline{\mathrm{u}}}$ & from $\Gamma^{\prime}$ \\
\hline 7 & $\neg(\mathrm{P} \rightarrow \mathrm{Q})_{\overline{\mathrm{s}}}$ & $2-6 \neg \mathrm{I}$ \\
\hline
\end{tabular}

$\Gamma^{\prime} \vdash_{\mathrm{NBx}}^{*} \neg(\mathrm{P} \rightarrow \mathrm{Q})_{\bar{s}}$; so by consistency, $\Gamma^{\prime} \nvdash_{\mathrm{NBx}}^{*}(\mathrm{P} \rightarrow \mathrm{Q})_{s}$. This is impossible; reject the assumption: if $\Gamma^{\prime} \vdash_{\mathrm{NBx}} A_{s}$ then $v_{w_{s}}(A)=1$. So $v_{w_{s}}(A)=1$ iff $\Gamma^{\prime} \vdash_{\mathrm{NBx}}^{*} A_{s}$.

(>) $A_{s}$ is $(P>Q)_{s}$. (i) Suppose $v_{w_{s}}(A)=1$ but $\Gamma^{\prime} \nvdash_{N B x}^{*} A_{s}$; then $v_{w_{\mathrm{s}}}(\mathrm{P}>\mathrm{Q})=1$ but $\Gamma^{\prime} \nvdash_{\mathrm{NBx}}^{*}(\mathrm{P}>\mathrm{Q})_{s}$. From the latter, by smaximality, $\Gamma^{\prime} \vdash_{\mathrm{NBx}}^{*} \neg(\mathrm{P}>\mathrm{Q})_{\bar{s}}$; so, since $\Gamma^{\prime}$ is a scapegoat set for $>$, there is some $y$ such that $\Gamma^{\prime} \vdash_{\mathrm{NBx}}^{*} P_{s / y}$, and $\Gamma^{\prime} \vdash_{\mathrm{NBx}}^{*} \neg Q_{\bar{y}}$; from the first of these, by construction, $\left\langle w_{s}, w_{y}\right\rangle \in R_{P}$; and from the second, by consistency, $\Gamma^{\prime} \nvdash_{\mathrm{NBx}}^{*} \mathrm{Q}_{y}$; so by assumption, $v_{w_{y}}(\mathrm{Q})=$ 0 ; so by $\mathrm{TB}(>), v_{w_{s}}(\mathrm{P}>\mathrm{Q})=0$. This is impossible; reject the assumption: if $v_{w_{s}}(A)=1$ then $\Gamma^{\prime} \vdash_{\mathrm{NBx}}^{*} A_{s}$.

(ii) Suppose $\Gamma^{\prime} \vdash_{\mathrm{NBx}}^{*} A_{s}$ but $v_{w_{s}}(A)=0$; then $\Gamma^{\prime} \vdash_{\mathrm{NBx}}^{*}(\mathrm{P}>\mathrm{Q})_{\mathrm{s}}$ but $v_{w_{s}}(\mathrm{P}>\mathrm{Q})=0$. From the latter, by $\mathrm{TB}(>)$, there is a $w_{\mathrm{t}}$ such that $\left\langle w_{s}, w_{t}\right\rangle \in R_{P}$, and $v_{w_{t}}(Q)=0$; so by assumption, $\Gamma^{\prime} \nvdash_{\mathrm{NBx}}^{*} \mathrm{Q}_{\mathrm{t}}$; so by s-maximality, $\Gamma^{\prime} \vdash_{\mathrm{NBx}}^{*} \neg \mathrm{Q}_{\overline{\mathrm{t}}}$. Since $\left\langle w_{\mathrm{s}}, w_{\mathrm{t}}\right\rangle \in \mathrm{R}_{\mathrm{P}}$, by construction, $\Gamma^{\prime} \vdash_{\mathrm{NBx}}^{*} \mathrm{P}_{\mathrm{s} / \mathrm{t}}$; so by reasoning as follows,

\begin{tabular}{|c|c|c|}
\hline I & $\Gamma^{\prime}$ & \\
\hline & $(\mathrm{P}>\mathrm{Q})_{\mathrm{s}}$ & $\mathrm{A}(c, \neg \mathrm{I})$ \\
\hline 3 & $P_{s / t}$ & from $\Gamma^{\prime}$ \\
\hline 4 & $Q_{t}$ & $2,3>E$ \\
\hline 5 & $\neg \mathrm{Q}_{\overline{\mathrm{t}}}$ & from $\Gamma^{\prime}$ \\
\hline 6 & $\neg(\mathrm{P}>\mathrm{Q})_{\overline{\mathrm{s}}}$ & $2-5 \neg \mathrm{I}$ \\
\hline
\end{tabular}

$\Gamma^{\prime} \vdash_{\mathrm{NBx}}^{*} \neg(\mathrm{P}>\mathrm{Q})_{\bar{s}}$; so by consistency, $\Gamma^{\prime} \nvdash_{\mathrm{NBx}}^{*}(\mathrm{P}>\mathrm{Q})_{s}$. This is impossible; reject the assumption: if $\Gamma^{\prime} \vdash_{\mathrm{NBx}} A_{s}$ then $v_{w_{s}}(A)=1$. So $v_{w_{s}}(A)=1$ iff $\Gamma^{\prime} \vdash_{\mathrm{NB} x}^{*} A_{s}$.

For any $A_{s}, v_{w_{s}}(A)=1$ iff $\Gamma^{\prime} \vdash_{\mathrm{NBx}}^{*} A_{s}$.

L8.9 If $\Gamma_{0}$ is consistent, then $\mathrm{I}_{\mathrm{B}_{x}}$ constructed as above is a $B x$ interpretation.

In each case, we need to show that relevant constraints are met. Suppose $\Gamma_{0}$ is consistent. By L8.7 $\Gamma^{\prime}$ is a scapegoat set for C9/Cio and Ci2 in those systems.

(NC) Suppose $\left\langle w_{0}, w_{s}, w_{t}\right\rangle \in R$; then by construction, $\Gamma^{\prime} \vdash_{\mathrm{NBx}}^{*}$ 0.s.t; so by $\mathrm{oE}, \Gamma^{\prime} \vdash_{\mathrm{NBx}}^{*} \mathrm{~s} \simeq \mathrm{t}$; so by construction, $w_{\mathrm{s}}=w_{\mathrm{t}}$. Suppose $w_{\mathrm{s}}=$ $w_{\mathrm{t}}$; then by construction, $\Gamma^{\prime} \vdash_{\mathrm{NBx}}^{*} \mathrm{~s} \simeq \mathrm{t}$; so by oI, $\Gamma^{\prime} \vdash_{\mathrm{NBx}}^{*}$ 0.s.t; 
so by construction, $\left\langle w_{0}, w_{s}, w_{t}\right\rangle \in R$. So $\left\langle w_{0}, w_{s}, w_{t}\right\rangle \in R$ iff $w_{s}=w_{\mathrm{t}}$; and, since $\mathrm{N}=\left\{w_{0}\right\}, \mathrm{NC}$ is satisfied.

(C8) If C8 is in $B x$, then AM8 is in NBx. Suppose $\left\langle w_{s}, w_{t}, w_{u}\right\rangle \in R$; then by construction, $\Gamma^{\prime} \vdash_{\mathrm{NBx}}^{*}$ s.t.u; so by AM8, $\Gamma^{\prime} \vdash_{\mathrm{NBx}}^{*}$ s. $\bar{u} . \bar{t}$; so by construction, $\left\langle w_{s}, w_{\overline{\mathrm{u}}}, w_{\overline{\mathrm{t}}}\right\rangle \in \mathrm{R}$; so by construction, $\left\langle w_{s}, w_{\mathrm{u}}^{*}, w_{\mathrm{t}}^{*}\right\rangle \in$ $R$. So C8 is satisfied.

(C9/ro) Suppose there is a $w_{\mathfrak{u}}$ such that $\left\langle w_{s}, w_{\mathfrak{t}}, w_{\mathfrak{u}}\right\rangle \in \mathrm{R}$ and $\left\langle w_{\mathfrak{u}}, w_{v}\right.$, $\left.w_{w}\right\rangle \in R$; then by construction, $\Gamma^{\prime} \vdash_{\mathrm{NBx}}^{*}$ s.t.u and $\Gamma^{\prime} \vdash_{\mathrm{NBx}}^{*}$ u.v.w; so, since $\Gamma^{\prime}$ is a C9/Cio scapegoat set, there is a $y$ such that $\Gamma^{\prime} \vdash_{\mathrm{NBx}}^{*}$ s.v.y and $\Gamma^{\prime} \vdash_{\mathrm{NBx}}^{*}$ t.y.w, and there is a $z$ such that $\Gamma^{\prime} \vdash_{\mathrm{NBx}}^{*}$ t.v. $z$ and $\Gamma^{\prime} \vdash_{\mathrm{NBx}}^{*}$ s.z.w; so by construction, $\left\langle w_{s}, w_{v}, w_{y}\right\rangle \in R$, $\left\langle w_{\mathrm{t}}, w_{\mathrm{y}}, w_{w}\right\rangle \in \mathrm{R},\left\langle w_{\mathrm{t}}, w_{v}, w_{z}\right\rangle \in \mathrm{R}$ and $\left\langle w_{\mathrm{s}}, w_{z}, w_{w}\right\rangle \in \mathrm{R}$. So $\mathrm{C}_{9}$ and $\mathrm{Cro}_{\mathrm{o}}$ are satisfied.

(Ci2) Similarly.

(C13) If $\mathrm{C}_{13}$ is in $B x$, then $\mathrm{AM}_{13}$ is in $N B x$. Suppose $\left\langle w_{s}, w_{t}, w_{\mathfrak{u}}\right\rangle \in \mathrm{R}$ and $\left\langle w_{\mathfrak{u}}, w_{v}, w_{w}\right\rangle \in R$; then by construction, $\Gamma^{\prime} \vdash_{\mathrm{NBx}}^{*}$ s.t.u and $\Gamma^{\prime} \vdash_{\mathrm{NBx}}^{*}$ u.v.w; so by $\mathrm{AM}_{3}, \Gamma^{\prime} \vdash_{\mathrm{NBx}}^{*}$ s.v.w; so by construction, $\left\langle w_{s}, w_{v}, w_{w}\right\rangle \in \mathrm{R}$. So $\mathrm{Cr}_{3}$ is satisfied.

( $\preceq$ ) If $(\preceq)$ is in $B x$, then $A M \preceq$ is in $N B x$. (i) Suppose $\left\langle w_{s}, w_{t}, w_{\mathfrak{u}}\right\rangle \in R$ and $v_{w_{s}}(p)=1$; then by construction, $\Gamma^{\prime} \vdash_{\mathrm{NBx}}^{*}$ s.t.u and $\Gamma^{\prime} \vdash_{\mathrm{NBx}}^{*} p_{s}$; so by $\mathrm{AM} \preceq, \Gamma^{\prime} \vdash_{\mathrm{NBx}}^{*} p_{u}$; so by construction, $v_{w_{u}}(p)=1$. (ii) Suppose $\left\langle w_{s}, w_{t}, w_{u}\right\rangle \in R$ and $v_{w_{u}^{*}}(p)=1$; then by construction, $v_{w_{\overline{\mathrm{u}}}}(p)=1$ so by construction again, $\Gamma^{\prime} \vdash_{\mathrm{NBx}}^{*}$ s.t.u and $\Gamma^{\prime} \vdash_{\mathrm{NBx}}^{*} p_{\overline{\mathrm{u}}}$; so by $\mathrm{AM} \preceq, \Gamma^{\prime} \vdash_{\mathrm{NBx}}^{*} p_{\bar{s}}$; so by construction, $v_{w_{\bar{s}}}(p)=1$; and by construction again, $v_{w_{s}^{*}}(p)=1$. $\mathrm{So}_{\mathrm{Cr}} 3$ is satisfied.

(I) If condition (I) is in $B x$, then AMPr is in $N B x$. Suppose $w_{\mathrm{t}} \in$ $f_{A}\left(w_{0}\right)$; then $\left\langle w_{0}, w_{t}\right\rangle \in R_{A}$; so by construction, $\Gamma^{\prime} \vdash_{\mathrm{NBx}}^{*} A_{0 / t}$; so by $A M P_{1}, \Gamma^{\prime} \vdash_{\mathrm{NBx}}^{*} A_{\mathrm{t}}$; so by L8.8, $v_{w_{\mathrm{t}}}(A)=1$; so $w_{\mathrm{t}} \in[A]$. So $\mathrm{f}_{A}\left(w_{0}\right) \subseteq[A]$ and $(\mathrm{I})$ is satisfied.

(2) If condition (2) is in $B x$, then $\mathrm{AMP}_{2}$ is in $N B x$. Suppose $w_{0} \in[A]$; then $v_{w_{0}}(A)=1$; so by L8.8, $\Gamma^{\prime} \vdash_{\mathrm{NBx}}^{*} A_{0}$; so by $\mathrm{AMP} 2, \Gamma^{\prime} \vdash_{\mathrm{NBx}}^{*}$ $A_{0 / 0}$; so by construction, $\left\langle w_{0}, w_{0}\right\rangle \in R_{A}$; so $w_{0} \in f_{A}\left(w_{0}\right)$ and (2) is satisfied.

MaP For any $w_{s} \in W$, set $m(s)=w_{s}$; otherwise $m(s)$ is arbitrary.

L8.Io If $\Gamma_{0}$ is consistent, then $v_{m}\left(\Gamma_{0}\right)=1$.

Reasoning parallel to that for $\mathrm{L} 2$. Io of $N K \alpha$.

Main result: Suppose $\Gamma \models_{\mathrm{B} x}$ A but $\Gamma \nvdash_{\mathrm{NBx}} A$. Then $\Gamma_{0} \models_{\mathrm{Bx}}^{*} A_{0}$ but $\Gamma_{0} \nvdash_{\mathrm{NBx}}^{*} A_{0}$. By $(\mathrm{DN})$, if $\Gamma_{0} \vdash_{\mathrm{NBx}}^{*} \neg \neg A_{0}$, then $\Gamma_{0} \vdash_{\mathrm{NBx}}^{*} A_{0}$; so $\Gamma_{0} \vdash_{\mathrm{NBx}}^{*} \neg \neg A_{0}$; so by L8.2, $\Gamma_{0} \cup\left\{\neg A_{\overline{0}}\right\}$ is consistent; so by L8.9 and L8.IO, there is a $B x$ interpretation with $v$ and 
$m$ constructed as above such that $v_{m}\left(\Gamma_{0} \cup\left\{\neg A_{\overline{0}}\right\}\right)=1$; so $v_{m}(\overline{0})(\neg A)=1$; so by construction, $v_{\mathrm{m}_{0}^{*}}(\neg A)=1$; so by $\operatorname{TB}(\neg), v_{\mathrm{m}(0)}(A)=0$; so $v_{\mathrm{m}}\left(\Gamma_{0}\right)=1$ and $v_{m(0)}(A)=0$; so by $\mathrm{VBx}^{*}, \Gamma_{0} \not \nvdash_{\mathrm{Bx}}^{*} A_{0}$. This is impossible; reject the assumption: if $\Gamma \models_{B \mathrm{~B}} A$, then $\Gamma \vdash_{\mathrm{NBx}} A$.

\section{QUANTIFIED MODAL LOGICS: Fn $\alpha$}

Quantified modal logic raises many issues in the metaphysics of possible worlds and modality. As graphically exhibited by the nineteen (!) branches of a tree diagram on the second page of Garson's excellent survey [3], there are many issues and options for formal logic as well. This last section is a bare introduction to the topic. I exhibit a couple of concerns associated with "variable domains," and consider some ways free logic might be adapted in response. Access is constrained as for normal modal logics from before.

When one moves from ordinary sentential logic to quantified logic, one moves from a simple interpretation which assigns a truth value to each parameter, to interpretations which include a universe of objects, with assignments to constants and relation symbols. It is natural to think we could do something similar in the transition from sentential to quantified modal logic. Thus, for example, we might say an interpretation is $\langle W, U, D, R, v\rangle$ where $W$ is a set of worlds, $U$ a set of objects, $D$ a function from $W$ to subsets of $U, R$ a subset of $W^{2}$, and $v$ a function which assigns a member of $U$ to each constant symbol, and a subset of $U^{n}$ to each n-place relation symbol at each world. Then, intuitively, for $w \in W, \mathrm{D}(w)$ says which things exist in world $w$. And $v$ says which things are assigned to constants and to relation symbols at worlds. Thus, we might have $v(\mathrm{~b})=$ Bill, $v_{w}\left(\mathrm{H}^{1}\right)=\{$ Bill, Hill $\}$ and $v_{x}\left(\mathrm{H}^{1}\right)=\{\mathrm{Hill}$, Jill $\}$; so that $\mathrm{Hb}$ turns out true at $w$ but false at $x-$ and, depending on access, we could proceed in the usual way to say that $\diamond \mathrm{Hb}$ at some world, or whatever.

Variable Domains. Here is a first concern: It is natural to think that Bill does not exist at every world - that D varies from one world to the next. And it is natural to think that 'everybody is happy' should come out true at $w$ just when all the people at $w$ are happy, and 'somebody is happy' should come out true just when someone at $w$ is happy. For this, for evaluation at $w$, quantifiers need to be restricted to the members of $\mathrm{D}(w)$. So far, so good. But consider the following argument, proceeding by standard quantifier rules (with subscripts applied in the usual way).

\begin{tabular}{|c|c|}
\hline 0.1 & $\mathrm{~A}(\mathrm{~g}, \square \mathrm{I})$ \\
\hline$(b=b)_{1}$ & $=$ \\
\hline$\exists x(x=b)$ & 2 \\
\hline$\square \exists x(x=b)_{0}$ & $\mathrm{I}-3$ \\
\hline
\end{tabular}

It is thus (apparently) a theorem that Bill exists at every accessible world so that Bill turns out to be a necessary being. Theological concerns to the side, something seems to have gone awry: for we began with precisely the 
assumption that Bill does not exist at every world.

Though its original motivation is not from possible worlds, quantified free logic is designed to accommodate interpretations with a universe $U$ of objects greater than the domain $\mathrm{D}$ over which quantifiers range. The idea seems to have been that there are objects which do not exist (Pegasus, or the like). Whatever sense is to be made of this, from our assumptions, there would seem to be a straightforward application to the modal context, where Bill is a member of some, but not every D. To accommodate this sort of thing, relative to the classical case, free logic imposes constraints on the quantifier rules. We may thus introduce a predicate $E$ for existence, with quantifier rules as follows,
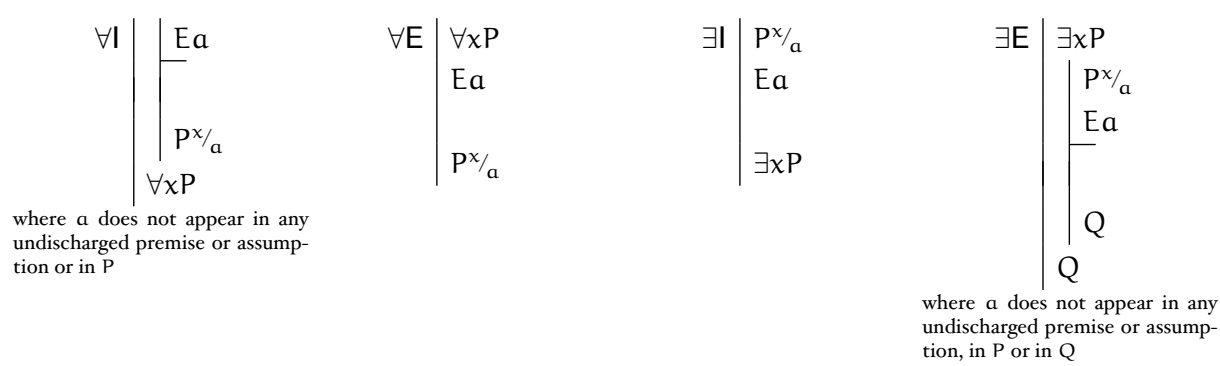

Then $\exists \mathrm{I}$ in our problematic derivation is blocked, insofar as $E b$ is not available. Of course, we might reason along the following lines,

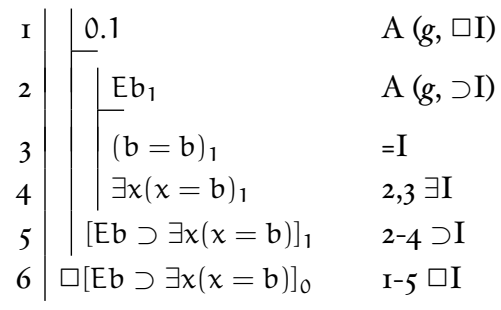

for the result that, necessarily, if Bill exists, then something is identical to Bill. But this seems right. So, subject to details, we seem to have the makings of a reasonable way out. (Notice that we have already seen a version of free logic for $\square$ and $\diamond$ as quantifiers over subscripts. Thus, where we see s.t as a sort of existence claim, these rules for $\forall$ and $\exists$ appear as parallel to ones we have seen for $\square$ and $\diamond$. In the modal case, we require the existence constraint insofar as the domain of worlds to which a given $w$ has access may turn out to be empty.)

$\mathrm{De} R e / \mathrm{De}$ Dicto. Much philosophical debate surrounds de re as opposed to de dicto modality. Formally an expression is de re (of the thing) iff a subformula of it has a constant or free variable in the scope of a modal operator. And expression is de dicto (of the saying) iff it is not de re. Thus, for example, $\square \mathrm{Hb}$ and $\exists \mathrm{x} \square \mathrm{Hx}$ are de re; $\square \exists \mathrm{xH}$ is de dicto. (Discussion of quantifying in by Quine and others is of de re modality, insofar as the quantifier reaches across the modal operator.) Evaluation of $\square \exists \mathrm{xH} x$ seems straightforward enough: $\square \exists \mathrm{xH}$ should be true just in case there is no accessible $w \in W$ such that $v_{w}(\mathrm{H})$ is empty. But consider $\square \mathrm{Hb}$; this will be true just in case Bill is in the extension of $\mathrm{H}$ at 
every accessible world. Similarly, $\exists x \square \mathrm{H} x$ will be true at $w$ just in case there is something in $w$ such that $i t$ is in the extension of $\mathrm{H}$ at every accessible world. Perhaps it is intuitive that a given thing could have been different ways, and so appears in different worlds. Even so, there are serious philosophical questions about how a thing has its modal features, and so appears in the worlds it does. ${ }^{8}$

Suppose we allow that a thing may appear in different worlds. As soon as we set things up this way, another problem emerges for variable domains. We have said that $\square \mathrm{Hb}$ will be true just in case Bill is in the extension of $\mathrm{H}$ at every accessible world. But Bill does not exist at every accessible world - and how are we to evaluate $\mathrm{Hb}$ at worlds where Bill does not exist (and similarly for $\exists x \mathrm{Hx}$ )? Here are three responses: (i) A standard response, perhaps because it is technically straightforward, is to say that non-existence at a world need not prevent a thing's being in the extension of a predicate there - to let $v_{w}(\mathrm{H})$ be any subset of $U$. Another reaction is to deny that an object can be in the extension of (ordinary) predicates at a world where it does not exist - this is to restrict $v_{w}(\mathrm{H})$ to subsets of $\mathrm{D}(w)$. Then (iia) we might let $\mathrm{Hb}$ be false at worlds where Bill does not exist. Alternatively, (iib), along lines from previous sections, we might say $\mathrm{Hb}$ is neither true nor false at worlds where Bill does not exist. Options (iia) and (iib) seem compatible with "serious actualism" as defended by Alvin Plantinga, though (iia) is like the one he explicitly endorses. ${ }^{9}$ In the following, I develop a version of free quantified modal logic compatible with any of these options - and fairly fine-grained combinations of them as well. If options are limited to just (i), or to just (i) and (iia), the logic remains classical, and obvious simplifications are possible. It is left as an exercise to work out the details of such simplifications.

\section{I LANGUAGE / SEMANTIC NOTIONS}

LFN $\alpha$ The vocabULARY consists of variables $x_{1}, x_{2} \ldots$; constants $c_{1}, c_{2} \ldots$; operators $\neg, \wedge, \forall, \square$; and relation symbols, $E,=, R_{1}^{1}, R_{2}^{1} \ldots, R_{1}^{2}, R_{2}^{2} \ldots$, etc. The number of "places" in a relation symbol is indicated by superscript, where ' $=$ ' is always two-place, and 'E' one-place. Any variable or constant is a TERM. If $Q^{n^{n}} t_{1} \ldots t_{n}$ is a $n$-place relation symbol followed by $n$ terms, it is a FORMULA. If $x$ is a variable and $A$ and $B$ are formulas, then $\neg A,(A \wedge B), \forall x A$ and $\square A$ are formulas. Variables are bound and free in the usual way. $A$ is a SENTENCE iff it is a formula with no free variables. We allow overlines as before, and the usual abbreviations, including $\vee, \supset, \equiv, \exists$, and $\diamond$.

IFN $\alpha$ An interpretation is $\langle W, U, D, R, P, v\rangle$ where $W$ is a set of worlds, $U$ a set of objects, $D$ a function from $W$ to subsets of $U, R$ a subset of $\mathrm{W}^{2}$, and $v$ a function such that for any constant $\mathrm{c}, v(\mathrm{c}) \in \mathrm{U}$ and for

\footnotetext{
${ }^{8}$ The literature is immense. Quine's [7] is a classic. For a contribution of my own, see [ro].

${ }^{9}$ See, e.g., [5]. But compare his [6, n.3]
} 
any $n$-place relation symbol $\mathrm{Q}^{n}$ and $w \in \mathrm{W}, v_{w}\left(\mathrm{Q}^{\mathrm{n}}\right) \subseteq \mathrm{U}^{\mathrm{n}}$. $\mathrm{P}$ is a function that maps each $n$-place relation symbol $Q^{n}$ to some member of $\{0,1,2\}^{n}$; so, for example, $P$ might map some $Q^{4}$ to $\langle 1,0,0,2\rangle$. Where $\mathrm{P}\left(\mathrm{Q}^{\mathfrak{n}}\right)=\left\langle\mathrm{a}_{1} \ldots \mathrm{a}_{\mathfrak{n}}\right\rangle$, say $\mathrm{P}\left(\mathrm{Q}^{\mathfrak{n}}\right)_{i}=\mathrm{a}_{\mathrm{i}}$. Then we require as an existence (presupposition) requirement on $v$ that,

EP If $\left\langle u_{1} \ldots u_{n}\right\rangle \in v_{w}\left(Q^{n}\right)$, then for any $i, 1 \leqslant i \leqslant n$, if $P\left(Q^{n}\right)_{i} \geqslant 1$, then $u_{i} \in \mathrm{D}(w)$

Additionally, require that $\mathrm{P}(\mathrm{E})=\langle 0\rangle$ and $v_{w}(\mathrm{E})=\mathrm{D}(w)$; if $\mathrm{P}(=)=\langle 0,0\rangle$, then $v_{w}(=)=\{\langle\mathfrak{u}, \mathfrak{u}\rangle \mid \mathfrak{u} \in \mathbf{U}\}$, and otherwise $v_{w}(=)=\{\langle\mathfrak{u}, \mathfrak{u}\rangle \mid \mathfrak{u} \in \mathrm{D}(\mathfrak{w})\}$. In addition, where $\alpha$ is empty or some combination of the following,

$\eta \quad$ For any $x$, there is a $y$ such that $x R y \quad$ extendability

$\rho$ for all $x, x R x$ reflexivity

$\sigma \quad$ for all $x, y$, if $x R y$ then $y R x \quad$ symmetry

$\tau$ for all $x, y, z$, if $x R y$ and $y R z$ then $x R z \quad$ transitivity

and, as sample versions of the presupposition constraint, where $n$ is one of the following,

(o) For any relation symbol $\mathrm{Q}^{\mathrm{n}}$ and any $i, 1 \leqslant i \leqslant n, P\left(\mathrm{Q}^{\mathrm{n}}\right)_{i}=0$

(I) For any relation symbol $Q^{n}$ other than $E$ and any $i, 1 \leqslant i \leqslant n$, $\mathrm{P}\left(\mathrm{Q}^{\mathrm{n}}\right)_{i}=1$

(2) For any relation symbol $Q^{n}$ other than $E$ and any $i, 1 \leqslant i \leqslant n$, $\mathrm{P}\left(\mathrm{Q}^{\mathrm{n}}\right)_{i}=2$

$\langle W, U, D, R, P, v\rangle$ is an Fn $\alpha$ interpretation when $R$ meets the constraints from $\alpha$, and $P$ meets the constraint from $n$. Obviously, many other options are available for the constraints $\alpha$ and $n$.

Given an interpretation with its $\mathrm{P}$ and $v$, say $\left\langle\mathrm{u}_{1} \ldots \mathrm{u}_{n}\right\rangle \in \bar{v}_{w}\left(\mathrm{Q}^{n}\right)$ just in case either $\left\langle u_{1} \ldots u_{n}\right\rangle \in v_{w}\left(Q^{n}\right)$ or for some $i, 1 \leqslant i \leqslant n, P\left(Q^{n}\right)_{i}=2$ and $u_{i} \notin \mathrm{D}(w)$. When $n=0$ or $n=1, v_{w}\left(\mathrm{Q}^{n}\right)$ is the same as $\bar{v}_{w}\left(\mathrm{Q}^{\mathrm{n}}\right)$. But when $n=2$, for relation symbols other than $E, \bar{v}_{w}\left(Q^{n}\right)$ includes also any $n$-tuple with a member not in $\mathrm{D}(w)$.

A variable designation assignment $\delta$ assigns each variable a member of $\mathrm{U}$; $\delta[x \mid u]$ is like $\delta$ except that $x$ is assigned to $u$; corresponding to a variable assignment $\delta(\delta[x \mid u])$ the term assignment $\Delta(\Delta[x \mid u])$ is like $\delta$ $(\delta[x \mid u])$ for variables, and $v$ for constants. As before, define a function $h$ based on $v$, writing $h_{w}(/ A /) / / \delta=1$ to indicate that $/ A /$ is SATISFIED at $w$ on $h$ with variable assignment $\delta$.

HF For assignments to formulas,

(R) $h_{w}\left(/ Q^{n^{n}} t_{1} \ldots t_{t} /\right) / / \delta=1$ if $\left\langle\Delta\left(t_{1}\right) \ldots \Delta\left(t_{2}\right)\right\rangle \in / v /{ }_{w}\left(Q^{n}\right)$, and 0 otherwise. 
$(\neg) h_{w}(/ \neg A /) / / \delta=1$ if $h_{w}(\backslash A \backslash) / / \delta=0$, and 0 otherwise.

$(\wedge) h_{w}(/ A \wedge B /) / / \delta=1$ if $h_{w}(/ A /) / / \delta=1$ and $h_{w}(/ B /) / / \delta=1$, and 0 otherwise.

$(\forall) h_{w}(/ \forall x A /) / / \delta=1$ if for any $u \in D(w), h_{w}(/ A /) / / \delta[x \mid u]=1$, and 0 otherwise.

( $\square$ ) $h_{w}(/ \square A /) / / \delta=1$ if all $x \in W$ such that $w R x$ have $h_{x}(/ A /) / / \delta=$ 1 , and 0 otherwise.

$h_{w}(/ A /)=1(/ A /$ bolds on $h$ at $w)$ iff for any $\delta, h_{w}(/ A /) / / \delta=1$. And $h_{w}(\Gamma)=1$ iff for each $/ A / \in \Gamma, h_{w}(/ A /)=1$. Then, where the members of $\Gamma$ and $A$ are sentences,

$\mathrm{VF}_{\mathrm{N}} \alpha \Gamma \models_{\text {nn } \alpha} / \mathrm{A} /$ iff there is no $F n \alpha$ interpretation $\langle\mathrm{W}, \mathrm{U}, \mathrm{D}, \mathrm{R}, \mathrm{P}, v\rangle$ and $w \in$ W such that $h_{w}(\Gamma)=1$ and $h_{w}(/ A /)=0$.

Set relation symbol E to the side: Then for $F_{0} \alpha$ and $F_{I} \alpha$, we have $h_{w}\left(/ Q^{n^{n}} t_{1}\right.$ $\left.\ldots t_{n} /\right) / / \delta=1$ iff $\left\langle\Delta\left(t_{1}\right) \ldots \Delta\left(t_{n}\right)\right\rangle \in v_{w}\left(Q^{n}\right)$ - where, for $F_{I} \alpha$ each of $\Delta\left(t_{1}\right)$ $\ldots \Delta\left(t_{n}\right)$ is required to be in $D(w)$. For $F_{2} \alpha, h_{w}\left(Q^{n} t_{1} \ldots t_{n}\right) / / \delta=1$ if and only if $\left\langle\Delta\left(t_{1}\right) \ldots \Delta\left(t_{n}\right)\right\rangle \in v_{w}\left(Q^{n}\right)$ - where again, each of $\Delta\left(t_{1}\right) \ldots \Delta\left(t_{n}\right)$ is required to be in $\mathrm{D}(w)$; but $\mathrm{h}_{w}\left(\overline{\mathrm{Q}^{n} t_{1} \ldots t_{n}}\right) / / \delta=0$ iff $\left\langle\Delta\left(\mathrm{t}_{1}\right) \ldots \Delta\left(\mathrm{t}_{\mathrm{n}}\right)\right\rangle \notin v_{w}\left(\mathrm{Q}^{\mathrm{n}}\right)$ and each of $\Delta\left(t_{1}\right) \ldots \Delta\left(t_{n}\right)$ is in $D(w)$. So $P$ works as an existence presupposition function for (each place of) each relation symbol: on the Fo $\alpha$ option, there are no existence presuppositions; on the $F_{I} \alpha$ option, there is an existence requirement for truth but not falsity; with $F_{2} \alpha$, there is an existence requirement for both truth and falsity. Insofar as truth and non-falsity are matched for the Fo $\alpha$ and $F_{I} \alpha$ options, the logic is essentially classical. However, for $F_{2} \alpha$, since expressions may be neither true nor false (but never both), the logic is like $\mathrm{MK}_{3}$ from section 6 .

As an example of reasoning with these definitions, here is an argument to show, $\exists \mathrm{y} \diamond \neg \mathrm{Ey} \models_{F_{1 \alpha}} \neg \forall x \square \mathrm{Hx}$. We suppose derived clauses to HF are spelled out in the usual way.

Suppose $\exists y \diamond \neg E y \nvdash_{F 1 \alpha} \neg \forall x \square H x$; then by VFN $\alpha$ there is an $F_{I} \alpha$ interpretation $\langle\mathrm{W}, \mathrm{U}, \mathrm{D}, \mathrm{R}, \mathrm{P}, v\rangle$ and $w \in W$ such that $\mathrm{h}_{w}(\exists y \diamond \neg \mathrm{Ey})=1$ but $h_{w}(\neg \forall x \square H x)=0$; from the latter, there is a $\delta$ where $h_{w}(\neg \forall x \square H x) / / \delta=$ 0 ; then with the former, $h_{w}(\exists y \diamond \neg E y) / / \delta=1$. From this, by $\operatorname{HF}(\exists)$, there is some $u \in D(w)$ such that $h_{w}(\diamond \neg E y) / / \delta[y \mid u]=1$; so by $\operatorname{HF}(\diamond)$, there is some $a \in W$ such that $w R a$ and $h_{a}(\neg E y) / / \delta[y \mid u]=1$; so by $\operatorname{HF}(\neg), h_{a}(\overline{E y}) / / \delta[y \mid u]=0$; so by $\operatorname{HF}(R), \Delta[y \mid u](y) \notin \bar{v}_{a}(E)$; so by the construction of $\bar{v}, \Delta[y \mid u](y) \notin v_{a}(E)$; so $\Delta[y \mid u](y) \notin D(a)$; but $\Delta[y \mid u](y)=\delta[y \mid u](y)=u$; so $u \notin D(a)$. Since $h_{w}(\neg \forall x \square H x) / / \delta=$ 0 , by $\mathrm{HF}(\neg), h_{w}(\overline{\forall x \square \mathrm{Hx}}) / / \delta=1$; so by $\mathrm{HF}(\forall)$, for any $v \in \mathrm{D}(w)$, $h_{w}(\overline{\square \mathrm{Hx}}) / / \delta[x \mid v]=1$; so $h_{w}(\overline{\square \mathrm{Hx}}) / / \delta[x \mid u]=1$; so by $\mathrm{HF}(\square)$, for any $\mathrm{b} \in W$ such that $w \mathrm{Rb}, \mathrm{h}_{\mathrm{b}}(\overline{\mathrm{Hx}}) / / \delta[\mathrm{x} \mid \mathrm{u}]=1$; so $h_{\mathrm{a}}(\overline{\mathrm{Hx}}) / / \delta[\mathrm{x} \mid \mathrm{u}]=1$; so by 
$\mathrm{HF}(\mathrm{R}), \Delta[\mathrm{x} \mid \mathrm{u}](\mathrm{x}) \in \bar{v}_{\mathrm{a}}(\mathrm{H})$; so, since this is $F_{I} \alpha, \Delta[\mathrm{x} \mid \mathrm{u}](\mathrm{x}) \in v_{\mathrm{a}}(\mathrm{H})$; so by EP, $\Delta[x \mid u](x) \in D(a)$; but $\Delta[x \mid u](x)=\delta[x \mid u](x)=u$; so $u \in D(a)$. This is impossible; reject the assumption: $\exists y \diamond \neg \mathrm{Ey} \models_{\mathrm{F} 1 \alpha} \neg \forall \mathrm{x} \square \mathrm{H} x$.

The argument does not go through in Fo $\alpha$ insofar as we cannot move by EP from $\Delta[x \mid u](x) \in v_{a}(H)$ to $\Delta[x \mid u](x) \in D(a)$. It does not go through in $F_{2} \alpha$ because we cannot move from $\Delta[\mathrm{x} \mid \mathrm{u}](\mathrm{x}) \in \bar{v}_{\mathrm{a}}(\mathrm{H})$ to $\Delta[\mathrm{x} \mid \mathrm{u}](\mathrm{x}) \in v_{\mathrm{a}}(\mathrm{H})$. However, as one can show by parallel reasoning, $\exists y \diamond \neg E y \models_{F_{2 \alpha}} \overline{\neg \forall x \square \mathrm{Hx}}$.

\subsection{NATURAL DERIVATIONS: NFn $\alpha$}

Allow expressions with integer subscripts and overlines and, as before, expressions of the sort, s.t. Begin with a natural combination of rules from free logic with ones we have seen before, where rules for $\vee, \supset, \equiv, \exists$ and $\diamond$ are derived.

\begin{tabular}{|c|c|c|c|c|c|}
\hline $\mathrm{R}$ & $\begin{array}{l}/ \mathrm{P} / \mathrm{s} \\
/ \mathrm{P} / \mathrm{s}\end{array}$ & $\neg \mathrm{I}$ & $\begin{array}{l}\mid / \mathrm{P} / \mathrm{s} \\
\| \mathrm{Q} /{ }_{\mathrm{t}} \\
\|\neg \mathrm{Q}\|_{\mathrm{t}} \\
\backslash \neg \mathrm{P} \backslash_{\mathrm{s}}\end{array}$ & $\neg \mathrm{E}$ & $\begin{array}{l}\mid / \neg \mathrm{P} / \mathrm{s} \\
\| / \mathrm{Q} / \mathrm{t}_{\mathrm{t}} \\
\|\neg \mathrm{Q}\|_{\mathrm{t}} \\
\backslash \mathrm{P} \backslash_{\mathrm{s}}\end{array}$ \\
\hline$\wedge \mathrm{I}$ & $\begin{array}{l}/ \mathrm{P} / \mathrm{s} \\
/ \mathrm{Q} / \mathrm{s} \\
/ \mathrm{P} \wedge \mathrm{Q} / \mathrm{s}\end{array}$ & $\wedge \mathrm{E}$ & $\begin{array}{l}/ \mathrm{P} \wedge \mathrm{Q} / \mathrm{s} \\
/ \mathrm{P} / \mathrm{s}\end{array}$ & $\wedge \mathrm{E}$ & $\begin{array}{l}/ \mathrm{P} \wedge \mathrm{Q}^{\prime} \mathrm{s} \\
/ \mathrm{Q}^{\prime} \mathrm{s}\end{array}$ \\
\hline VI & $\begin{array}{l}/ \mathrm{P} / \mathrm{s} \\
/ \mathrm{P} \vee \mathrm{Q} / \mathrm{s}\end{array}$ & $\mathrm{VI}$ & $\begin{array}{l}/ \mathrm{P} / \mathrm{s} \\
/ \mathrm{Q} \vee \mathrm{P} / \mathrm{s}\end{array}$ & VE & $\begin{array}{l}/ \mathrm{P} \vee \mathrm{Q} / \mathrm{s} \\
\mid / \mathrm{P} / \mathrm{s}\end{array}$ \\
\hline اد & $\begin{array}{l}\left.\right|^{/ \mathrm{P} / \mathrm{s}} \\
\backslash \mathrm{Q} \backslash_{\mathrm{s}} \\
\backslash \mathrm{P} \supset \mathrm{Q} \backslash_{\mathrm{s}}\end{array}$ & $\supset \mathrm{E}$ & $\begin{array}{l}\backslash \mathrm{P} \supset \mathrm{Q} \backslash_{\mathrm{s}} \\
/ \mathrm{P} /_{\mathrm{s}} \\
\backslash \mathrm{Q} \backslash_{\mathrm{s}}\end{array}$ & & $\begin{array}{l}\mid / / \mathrm{R} / \mathrm{t} \\
\mid / \mathrm{Q} / \mathrm{s} \\
\left.\right|_{/ / \mathrm{R} / / \mathrm{t}}\end{array}$ \\
\hline$\equiv \mathrm{I}$ & $\begin{array}{l}\mid / \mathrm{P} / \mathrm{s} \\
\mid \mathrm{Q} \backslash_{\mathrm{s}} \\
\mid / \mathrm{Q} / \mathrm{s}\end{array}$ & $\equiv \mathrm{E}$ & $\begin{array}{l}\backslash \mathrm{P} \equiv \mathrm{Q} \backslash_{\mathrm{s}} \\
/ \mathrm{P} /_{\mathrm{s}} \\
\backslash \mathrm{Q} \backslash_{\mathrm{s}}\end{array}$ & $\equiv \mathrm{E}$ & $\begin{array}{l}\backslash \mathrm{P} \equiv \mathrm{Q} \backslash_{\mathrm{s}} \\
/ \mathrm{Q} / \mathrm{s} \\
\backslash \mathrm{P} \backslash_{\mathrm{s}}\end{array}$ \\
\hline
\end{tabular}




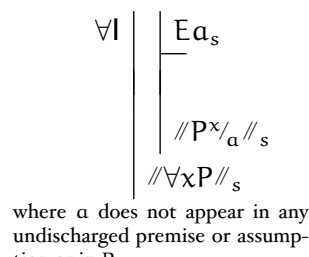

$$
\forall \mathrm{E} \mid \begin{aligned}
& / / \forall \times \mathrm{P} / / \mathrm{s} \\
& \mathrm{Ea}_{\mathrm{s}} \\
& / / \mathrm{Px} / \mathrm{a} / \mathrm{s}
\end{aligned}
$$

$\exists \mathrm{\exists l} \mid \begin{aligned} & / / \mathrm{P}^{\mathrm{x} / a} / / \mathrm{s} \\ & \mathrm{Ea}_{\mathrm{s}} \\ & / / \exists \mathrm{xP} / / \mathrm{s}\end{aligned}$

tion or in $\mathrm{P}$

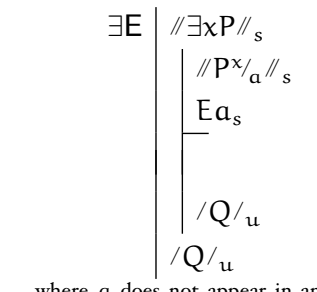

where a does not appear in an undischarged premise or assumption, in $\mathrm{P}$ or in $\mathrm{Q}$
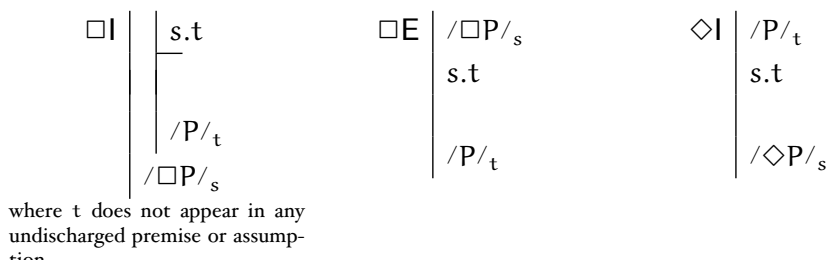

tion

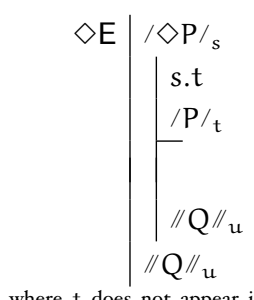

where $t$ does not appear in an undischarged premise or assumption and is not $u$
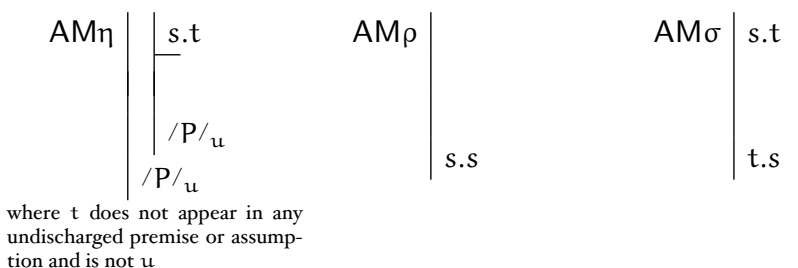

$\operatorname{AM\tau } \mid \begin{aligned} & \text { s.t } \\ & \text { t.u } \\ & \text { s.u }\end{aligned}$

Every subscript is 0 , appears in a premise, or in the $t$ place of an assumption for $\square \mathrm{I}, \diamond \mathrm{E}$ or $\mathrm{AM \eta}$. Now, for relation symbol $\mathrm{Q}^{n}$, let $\mathrm{P} 1\left[\mathrm{Q}^{n} t_{1} \ldots t_{n}\right]_{s}$ be the conjunction $\left(T \wedge E t_{a} \wedge \cdots \wedge E t_{b}\right)_{s}$ for each $t_{i}$ such that $P\left(Q^{n}\right)_{i} \geqslant 1$. And let $P 2\left[Q^{n} t_{1} \ldots t_{n}\right]_{s}$ be the conjunction $\left(T \wedge E t_{a} \wedge \ldots \wedge E t_{b}\right)_{s}$ for each $t_{i}$ such that $\mathrm{P}\left(\mathrm{Q}^{\mathrm{n}}\right)_{i}=2$. Note that $T_{s}$ can be asserted at any stage in a derivation. Then allow,

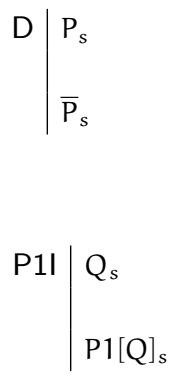

$$
=\mathrm{I} \mid \begin{aligned}
& \mathrm{P} 1[\mathrm{a}=\mathrm{a}]_{s} \\
& (\mathrm{a}=\mathrm{a})_{s}
\end{aligned}
$$$$
=\mathrm{E} \mid \begin{array}{l|l}
(\mathrm{a}=\mathrm{b})_{\mathrm{s}} & (\mathrm{b}=\mathrm{a})_{\mathrm{s}} \\
/ / \mathrm{Q} / / \mathrm{t} & / / \mathrm{Q} / \mathrm{t} \\
& / / \mathrm{Q} \mathrm{a} / \mathrm{b} / \mathrm{t} \\
\text { a single instance of } \mathrm{a} \text { replaced by } \mathrm{b} & / \mathrm{Q} / \mathrm{b} / / \mathrm{t}
\end{array}
$$$$
\mathrm{P} 2 \mathrm{E} \mid \begin{aligned}
& \mathrm{P} 2[\mathrm{Q}]_{s} \\
& \overline{\mathrm{Q}}_{\mathrm{s}} \\
& \mathrm{Q}_{\mathrm{s}}
\end{aligned}
$$

Where for $=E, P_{1} I, P_{2} I$ and $P_{2} E, Q$ is an atomic, $R^{n} a_{1} \ldots a_{n}$.

Notice that $\mathrm{P}_{2} \mathrm{E}$ is a constrained version of $(\mathrm{U})$ from section 6. Informally, where $P 1\left[Q^{n_{1}} t_{1} \ldots t_{n}\right]_{s}$ or $P 2\left[Q^{n} t_{1} \ldots t_{n}\right]_{s}$ is other than just $T$, let us drop $T$ for the equivalent conjunction. Then, in any case, $P 1(E)=P 2(E)=T$. Otherwise, in NFo $\alpha, P 1\left[\mathrm{Q}^{n_{1}} t_{1} \ldots t_{n}\right]=\mathrm{P} 2\left[\mathrm{Q}^{n} \mathrm{t}_{1} \ldots \mathrm{t}_{\mathrm{n}}\right]=T$. In $N F_{I} \alpha, \mathrm{P} 1\left[\mathrm{Q}^{\mathrm{n}_{1}} \mathrm{t}_{1} \ldots \mathrm{t}_{\mathrm{n}}\right]=$ 
$E t_{1} \wedge \ldots \wedge E t_{n}$ and $P 2\left[Q^{n} t_{1} \ldots t_{n}\right]=\top$. In $N F_{2} \alpha$, we have $P 1\left[Q^{n} t_{1} \ldots t_{n}\right]=$ $P 2\left[Q^{n} t_{1} \ldots t_{n}\right]=E t_{1} \wedge \ldots \wedge E t_{n}$. When $P 1\left[Q^{n} t_{1} \ldots t_{n}\right]=T$, the premise for $=I$ goes trivial, as does the conclusion from PII. Similarly, when P2 $\left[\mathrm{Q}^{n^{n}} t_{1} \ldots t_{n}\right]=$ $T$, the conclusion of $\mathrm{P}_{2} \mathrm{I}$ is trivial, and $\mathrm{P}_{2} \mathrm{E}$ works like (U) for the relevant atomic. Where the members of $\Gamma$ and $/ A /$ are sentences without subscripts, let $\Gamma_{0}$ be the members of $\Gamma$, each with subscript 0 . Then,

$N F N \alpha \Gamma \vdash_{N F n \alpha} / A /$ iff there is an NFn $\alpha$ derivation of $/ A / 0$ from the members of $\Gamma_{0}$.

Notice that our notions of validity are defined for sentences. We get the different derivation systems insofar as AM rules may differ, and insofar as P1[Q] and $\mathrm{P} 2[\mathrm{Q}]$ are different expressions. On occasion, arguments will go through no matter what presupposition constraints are in play. In this case, to show $\Gamma \vdash_{\mathrm{N} n \alpha \alpha} / \mathrm{A} /$, apply the rules so that they would apply no matter what the constraints are. Thus apply $=\mathrm{I}$ and $\mathrm{P}_{2} \mathrm{E}$ as though $\mathrm{P}\left(\mathrm{Q}^{\mathrm{n}}\right)_{i}$ is always 2 , and $\mathrm{P}_{\mathrm{I}} \mathrm{I}$ and $\mathrm{P}_{2} \mathrm{I}$ as though it is o (so the latter two rules effectively drop out).

As above, rules for $\vee, \supset, \equiv, \exists$ and $\diamond$ are derived. As examples, here are derivations for $\exists \mathrm{I}$ and $\exists \mathrm{E}$.
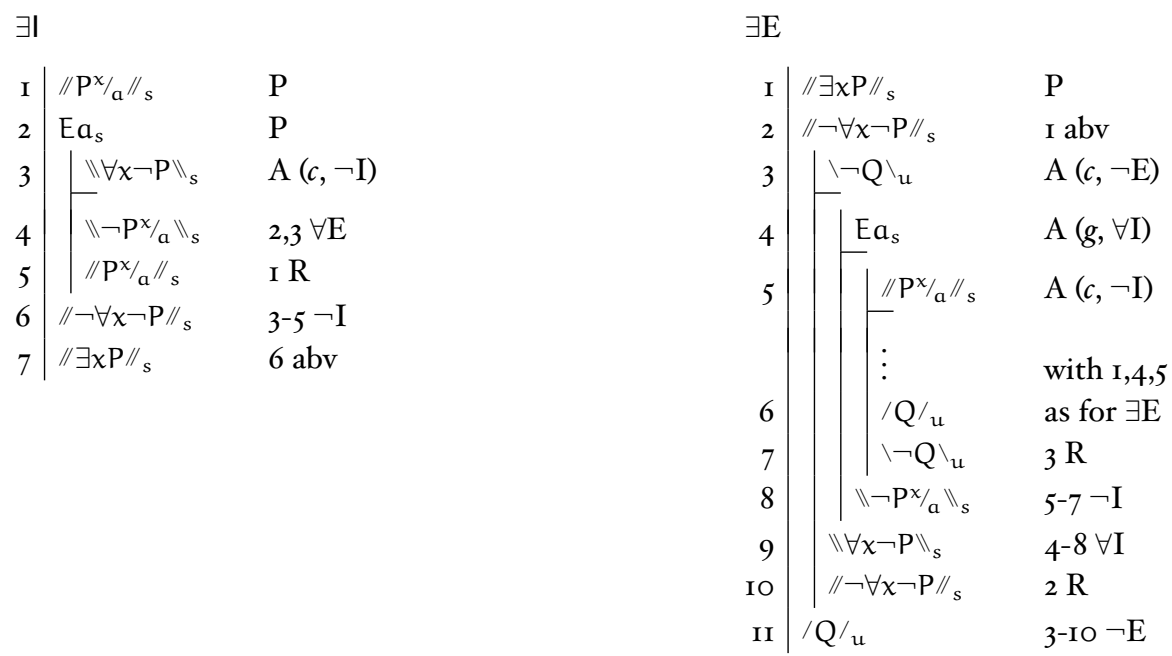

In addition, we allow standard two-way rules (including $\mathrm{MN}$ ) with overlines and subscripts constant throughout. Include among two-way rules,

$$
\text { QN } \begin{aligned}
\quad \forall x \mathrm{P} / \mathrm{s} & \triangleleft \triangleright / \neg \exists x \neg \mathrm{P} / \mathrm{s} & / \neg \forall \mathrm{xP} / \mathrm{s} \quad \triangleleft \triangleright / \exists x \neg \mathrm{P} / \mathrm{s} \\
/ \exists \mathrm{xP} / \mathrm{s} & \triangleleft \triangleright / \neg \forall x \neg \mathrm{P} / \mathrm{s} & / \neg \exists \mathrm{xP} / \mathrm{s} \quad \triangleleft \triangleright / \forall x \neg \mathrm{P} / \mathrm{s}
\end{aligned}
$$

Allow MT, NB and DS in the forms,

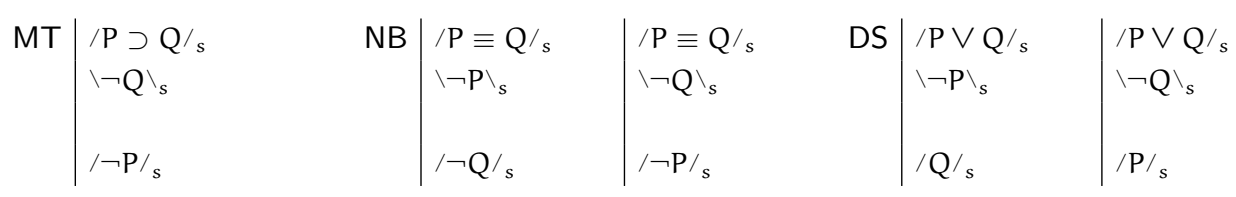


As further examples, here are some simple results emphasizing behavior for existence and identity.

$$
\begin{aligned}
& a=b \vdash_{\mathrm{NFO \alpha}} \square(a=b)
\end{aligned}
$$

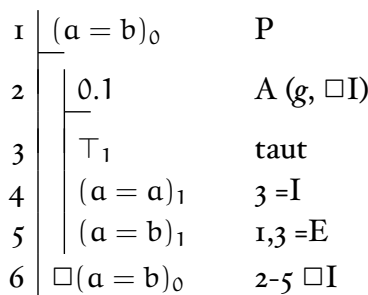

In NFo $\alpha$, the premise for $=\mathrm{I}$ is trivial. Notice that this derivation does not go through for $N F_{I} \alpha$ and $N F_{2} \alpha$, where =I requires a substantive premise. As above, we may, however show the following (and similarly for $N_{2} \alpha$ ),

\begin{tabular}{|c|c|c|}
\hline \\
\hline \multicolumn{3}{|c|}{$\begin{array}{l}=b \vdash_{N F 1 \alpha} \square[E a \supset(a=b)] \\
I \mid(a=b)_{0} \quad P\end{array}$} \\
\hline \multicolumn{3}{|c|}{ 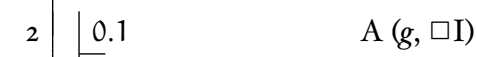 } \\
\hline 3 & $\overline{\mathrm{Ea}}_{1}$ & $\mathrm{~A}(\mathrm{~g}, \supset \mathrm{I})$ \\
\hline \multirow[b]{2}{*}{5} & $\top_{1}$ & taut \\
\hline & $\mathrm{Ea}_{1}$ & $3,4 \mathrm{P}_{2} \mathrm{E}$ \\
\hline 5 & $(E a \wedge E a)_{1}$ & $5,5 \wedge \mathrm{I}$ \\
\hline 7 & $(a=a)_{1}$ & $6=I$ \\
\hline 8 & $(a=b)_{1}$ & $\mathrm{I}, 7=\mathrm{E}$ \\
\hline \multirow{2}{*}{$\begin{array}{l}9 \\
0\end{array}$} & {$[E a \supset(a=b)]_{1}$} & $3^{-8} \supset \mathrm{I}$ \\
\hline & $\square[\mathrm{Ea}$ & 2-9 $\square \mathrm{I}$ \\
\hline
\end{tabular}

The premise for $\mathrm{P}_{2} \mathrm{E}$ is trivial, as always for relation symbol $\mathrm{E}$. But the premise for $=\mathrm{I}$ is not. In this case, the terms are the same so, following the rule (but dropping $T$ ), the required premise is obtained at (6). In these systems, then, if $a$ is equal to $b, a$ is essentially equal to $b$.

Here is a case considered semantically above, 


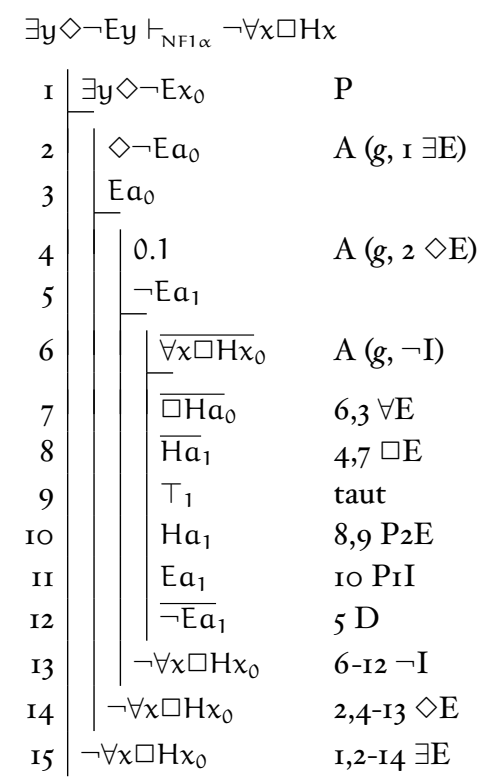

Notice that, in $N F_{2} \alpha$ we would not have (Io) since we do not have $E a_{1}$. And in NFo $\alpha$, (IO) would get us just $T_{1}$ instead of $E a_{1}$ (again with $T$ dropped) and we would not have the contradiction. As in the semantic case, though, we can show,

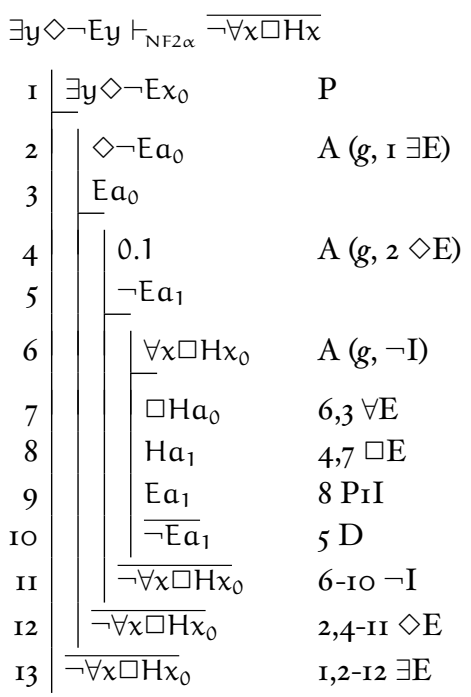

When there is a world where some a does not exist, on $F_{I} \alpha$, we can be sure that atomics go false for the thing at that world, so that the $\square$ goes false as well. On $F_{2} \alpha$, we can be sure that atomics are not true for the thing at that world, so that $\square$ is not true either. On Fo $\alpha$, there are no immediate consequences, insofar as atomics might go either way for the thing at that world.

Insofar as there is no parallel discussion for quantified modal logic in Priest's text, the following are offered as exercises which the student may find useful.

I. Produce an interpretation to show that, for any $n, \forall \forall_{\text {nрот }} \forall x \square Q x \supset \square \forall x Q x$. 
2. Produce an interpretation to show that, for any $n, \forall$ Fnрoт $_{\text {n }} \square \forall x \mathrm{Q} x \supset \forall x \square \mathrm{Q} x$.

3. The formulas in (I) and (2) are instances of the Barcan formula and converse Barcan formula respectively (after Ruth Barcan Marcus). They play an important role in discussions of quantified modal logic, especially related to issues with which we began. Show that the formulas are valid in a system Fnc which is like $F n \rho \sigma \tau$ except that it includes the constraint (c) that for any $a, b \in W, D(a)=D(b)$.

4. Give derivations to show each of the following.
a. $\square \forall x A x \vdash_{N F n \alpha} \forall x \square(E x \supset A x)$
b. $\exists x \forall y \square A x y \vdash_{\mathrm{NF} 1 \alpha} \square \exists x \exists y A x y$
c. $\vdash_{\mathrm{NFn} p} \forall x \square(A x \wedge B x) \supset \forall y A y$
d. $\vdash_{\text {Nhro }}(\diamond \forall x A x \wedge \square \exists x B x) \supset \diamond \exists x(A x \wedge B x)$
e. $\vdash_{\text {Nhn } \alpha} \square \forall x(A x \vee B x) \supset(\square \forall x A x \vee \diamond \exists x B x)$
f. $\vdash_{\mathrm{NFI \alpha}}(\diamond \exists x \square A x \wedge \square \forall x \diamond B x) \supset \diamond \exists x \diamond(A x \wedge B x)$
g. $\forall x \neg \diamond A x \supset \square \forall x \neg A x \vdash_{\text {NFn } \alpha} \diamond \exists x A x \supset \exists x \diamond A x$
h. $\diamond \exists x A x \supset \exists x \diamond A x \vdash_{\mathrm{NFn} \alpha} \forall x \neg \diamond A x \supset \neg \diamond \exists x A x$
i. $\exists x \diamond \neg A x \vdash_{N F 2 \alpha} \diamond \exists x \neg A x$
j. $\vdash_{\mathrm{NFO \alpha}} \forall x \square(A x \vee \neg A x)$

5. (i) Suppose no $P\left(Q^{n}\right)_{i}=2$. Provide a revised version of our derivation rules which takes advantage of this simplification. Hint: it is possible to do away with overlines altogether. Why? (ii) Suppose $\mathrm{P}\left(\mathrm{Q}^{\mathfrak{n}}\right)_{i}$ is always o. Provide a version of our derivation rules which takes advantage of this additional simplification.

\section{$9 \cdot 3$ SOUNDNESS AND COMPLETENESS}

Preliminaries: Begin with generalized notions of validity. Given any model $\langle W, U, D, R, P, v\rangle$, let $m$ be a map from subscripts into $W$. Then say $\langle W, U$, $\mathrm{D}, \mathrm{R}, \mathrm{P}, v\rangle_{\mathrm{m}}$ is $\langle\mathrm{W}, \mathrm{U}, \mathrm{D}, \mathrm{R}, \mathrm{P}, v\rangle$ with map $\mathrm{m}$. Then, where $\Gamma$ is a set of expressions of our language for derivations, $h_{m}(\Gamma)=1$ iff for each $/ A / s \in \Gamma$, $h_{m(s)}(/ A /)=1$ and for each $s . t \in \Gamma,\langle m(s), m(t)\rangle \in R$. Now expand notions of validity for subscripts, and alternate expressions as indicated in double brackets. Where the formulas in $\Gamma$ and $A$ are sentences,

$\mathrm{VF}_{\mathrm{N}} \alpha^{*} \Gamma \models_{\text {in } \alpha}^{*} / \mathrm{A} / \mathrm{s} \llbracket s . t \rrbracket$ iff there is no $F n \alpha$ interpretation $\langle\mathrm{W}, \mathrm{U}, \mathrm{D}, \mathrm{R}, \mathrm{P}, v\rangle_{\mathrm{m}}$ such that $h_{m}(\Gamma)=1$ but $h_{m(s)}(/ A /)=0 \llbracket\langle m(s), m(t) \notin R \rrbracket$.

$\mathrm{NFN}^{*} \Gamma \vdash_{\mathrm{NFn} \alpha}^{*} / \mathrm{A} / \mathrm{s} \llbracket$ s.t $\rrbracket$ iff there is an NFn $\alpha$ derivation of $/ \mathrm{A} / \mathrm{s} \llbracket$ s.t $\rrbracket$ from the members of $\Gamma$. 
These notions reduce to the standard ones when all the members of $\Gamma$ and A have subscript 0 . As usual, for the following, cases omitted are left to the reader.

THEOREM 9.I NFn $\alpha$ is sound: If $\Gamma \vdash_{\mathrm{NFn} \alpha} / \mathrm{A} /$ then $\Gamma \models_{\mathrm{n} \alpha} / \mathrm{A} /$.

L9.I If $\Gamma \subseteq \Gamma^{\prime}$ and $\Gamma \models_{\text {Fn } \alpha}^{*} / \mathrm{P} / \mathrm{s} \llbracket$ s.t $\rrbracket$, then $\Gamma^{\prime} \models_{\text {Tn } \alpha}^{*} / \mathrm{P} / \mathrm{s} \llbracket$ s.t $\rrbracket$.

L9.2 If $\delta$ and $\delta^{\prime}$ agree on their assignments to variables free in $/ \mathrm{P} /$ then $h_{w}(/ \mathrm{P} /) / / \delta=h_{w}(/ \mathrm{P} /) / / \delta^{\prime}$.

L9.3 If $v$ and $v^{\prime}$ differ at most in assignments to terms that do not occur in $/ \mathrm{P} /$, then for the corresponding $h$ and $h^{\prime}, h_{w}(/ \mathrm{P} /) / / \delta=h_{w}^{\prime}(/ \mathrm{P} /) / / \delta$. Corollary: $h_{w}(/ \mathrm{P} /)=h_{w}^{\prime}(/ \mathrm{P} /)$.

L9.4 If $\Delta(a)=u$, then $h_{w}\left(\backslash P^{x} / a \backslash\right) / / \delta=h_{w}(\backslash P \backslash) / / \delta[x \mid u]$.

Demonstrations for L9.I - L9.4 are all on the model of parallel results from classical logic.

Main result: For each line in a derivation let $\mathcal{P}_{i}$ be the expression on line $i$ and $\Gamma_{i}$ be the set of all premises and assumptions whose scope includes line $i$. We set out to show "generalized" soundness: if $\Gamma \vdash_{\mathrm{Nnn} \alpha}^{*} \mathcal{P}$ then $\Gamma \models_{\text {Fn } \alpha}^{*} \mathcal{P}$. As above, this reduces to the standard result when $\mathcal{P}$ and all the members of $\Gamma$ are without overlines and have subscript 0 . Suppose $\Gamma \vdash_{\mathrm{Nfn} \alpha}^{*} \mathcal{P}$. Then there is a derivation of $\mathcal{P}$ from premises in $\Gamma$ where $\mathcal{P}$ appears under the scope of the premises alone. By induction on line number of this derivation, we show that for each line $i$ of this derivation, $\Gamma_{i} \models_{\text {nn } \alpha}^{*} \mathcal{P}_{i}$. The case when $\mathcal{P}_{i}=\mathcal{P}$ is the desired result.

Basis: $\mathcal{P}_{1}$ is a premise or an assumption $/ A / s \llbracket$ s.t $\rrbracket$. Then $\Gamma_{1}=\{/ A / s\} \llbracket\{s . t\} \rrbracket$; so for any $\langle W, U, D, R, P, v\rangle_{m}$, we have $h_{m}\left(\Gamma_{1}\right)=1$ iff $h_{m(s)}(/ A /)=1$ $\llbracket\langle m(s), m(t)\rangle \in R \rrbracket$; so there is no $\langle W, U, D, R, P, v\rangle_{m}$ where $h_{m}\left(\Gamma_{1}\right)=1$ but $h_{m(s)}(/ A /)=0 \llbracket\langle m(s), m(t)\rangle \notin R \rrbracket$. So by $\operatorname{VFN}_{N} \alpha^{*}$, it follows that $\Gamma_{1} \models_{\text {ma }}^{*} / A / s \llbracket$ s.t $\rrbracket$, where this is just to say, $\Gamma_{1} \models_{\text {ma }}^{*} \mathcal{P}_{1}$.

Assp: For any $i, 1 \leqslant i<k, \Gamma_{i} \models_{\text {m } \alpha}^{*} \mathcal{P}_{i}$.

Show: $\Gamma_{\mathrm{k}} \models_{\text {Fi } \alpha}^{*} \mathcal{P}_{\mathrm{k}}$.

$\mathcal{P}_{k}$ is either a premise, an assumption, or arises from previous lines by $\mathrm{R}$, $\wedge \mathrm{I}, \wedge \mathrm{E}, \neg \mathrm{I}, \neg \mathrm{E}, \forall \mathrm{I}, \forall \mathrm{E}, \square \mathrm{I}, \square \mathrm{E}, \mathrm{D},=\mathrm{I},=\mathrm{E}, \mathrm{P}_{\mathrm{I}}, \mathrm{P}_{2} \mathrm{I}, \mathrm{P}_{2} \mathrm{E}$ or, depending on the system, $A M \eta, A M \rho, A M \sigma$, or $A M \tau$. If $\mathcal{P}_{k}$ is a premise or an assumption, then as in the basis, $\Gamma_{k} \models_{\text {nn } \alpha}^{*} \mathcal{P}_{k}$. So suppose $\mathcal{P}_{k}$ arises by one of the rules.

(R) 
( $\mathrm{I})$ If $\mathcal{P}_{\mathrm{k}}$ arises by $\wedge \mathrm{I}$, then the picture is like this,

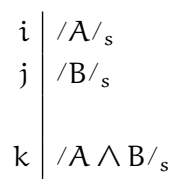

where $i, j<k$ and $\mathcal{P}_{k}$ is $/ A \wedge B / s$. By assumption, $\Gamma_{i} \models_{\text {nn } \alpha}^{*} / A / s$ and $\Gamma_{\mathrm{j}} \models_{\text {nn }}^{*} / \mathrm{B} / \mathrm{s}$; but by the nature of access, $\Gamma_{\mathrm{i}} \subseteq \Gamma_{\mathrm{k}}$ and $\Gamma_{\mathrm{j}} \subseteq \Gamma_{\mathrm{k}}$; so by

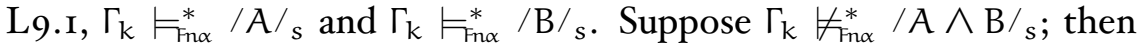
by $\mathrm{VFN}_{\mathrm{N}} \alpha^{*}$, there is an $F n \alpha$ interpretation $\langle\mathrm{W}, \mathrm{U}, \mathrm{D}, \mathrm{R}, \mathrm{P}, v\rangle_{\mathrm{m}}$ such that $h_{m}\left(\Gamma_{k}\right)=1$ but $h_{m(s)}(/ A \wedge B /)=0$. From the latter, there is a $\delta$ such that $h_{m(s)}(/ A \wedge B /) / / \delta=0$. From the former, by $\operatorname{VF}_{N} \alpha^{*}, h_{m(s)}(/ A /)=$ 1 and $h_{m(s)}(/ B /)=1$; so $h_{m(s)}(/ A /) / / \delta=1$ and $h_{m(s)}(/ B /) / / \delta=1$; so by $\operatorname{HF}(\wedge), h_{m(s)}(/ A \wedge B /) / / \delta=1$. This is impossible; reject the

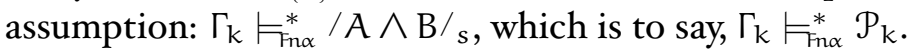

$(\wedge \mathrm{E})$

$(\neg \mathrm{I})$ If $\mathcal{P}_{k}$ arises by $\neg \mathrm{I}$, then the picture is like this,

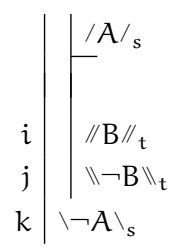

where $i, j<k$ and $\mathcal{P}_{k}$ is $\backslash \neg A \backslash_{s}$. By assumption, $\Gamma_{i} \models_{\text {tn } \alpha}^{*} / / B / / t$ and $\Gamma_{j} \models_{\text {tin } \alpha}^{*}$ $\|\neg B\|_{t}$; but by the nature of access, $\Gamma_{i} \subseteq \Gamma_{k} \cup\{/ A / s\}$ and $\Gamma_{j} \subseteq \Gamma_{k} \cup$ $\{/ A / s\}$; so by L9.I, $\Gamma_{k} \cup\{/ A / s\} \models_{\text {th } \alpha}^{*} / / B / /{ }_{t}$ and $\Gamma_{k} \cup\{/ A / s\} \models_{\text {nn } \alpha}^{*}\|\neg B\|_{t}$. Suppose $\Gamma_{\mathrm{k}} \not \nvdash_{\text {,n } \alpha}^{*} \backslash \neg A \backslash_{s}$; then by $V_{F} \alpha^{*}$, there is an $F n \alpha$ interpretation $\langle\mathrm{W}, \mathrm{U}, \mathrm{D}, \mathrm{R}, \mathrm{P}, v\rangle_{\mathrm{m}}$ such that $h_{\mathrm{m}}\left(\Gamma_{\mathrm{k}}\right)=1$ but $h_{\mathrm{m}(\mathrm{s})}(\backslash \neg A \backslash)=0$; from the latter, there is some $\delta$ such that $h_{m(s)}(\backslash \neg A \backslash) / / \delta=0$; so by $\operatorname{HF}(\neg)$, $h_{m(s)}(/ A /) / / \delta=1$; but a derivation is a sequence of sentences; so $\neg A$ and so $A$ have no free variables; so by L9.2, for any $\delta^{\prime}, h_{m(s)}(/ A /) / / \delta^{\prime}=$ 1 ; so $h_{m(s)}(/ A /)=1$; so $h_{m}\left(\Gamma_{k}\right)=1$ and $h_{m(s)}(/ A /)=1$; so $h_{m}\left(\Gamma_{k} \cup\right.$ $\{/ A / s\})=1$; so by VFN $\alpha^{*}, h_{m(t)}(/ / B / /)=1$ and $h_{m(t)}(\|\neg B\|)=1$; so for any $\delta, h_{m(t)}(/ / B / /) / / \delta=1$ and $h_{m(t)}(\|\neg B\|) / / \delta=1$; from the latter, by $\operatorname{HF}(\neg), h_{m(t)}(/ / B / /) / / \delta=0$. This is impossible; reject the

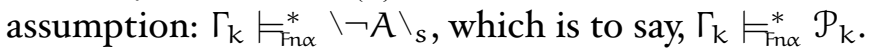

$(\neg \mathrm{E})$

$(\forall \mathrm{I})$ If $\mathcal{P}_{k}$ arises by $\forall \mathrm{I}$, then the picture is like this,

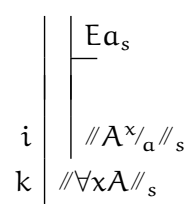


where $i<k$, a does not appear in any member of $\Gamma_{k}$ (in any undischarged premise or assumption) or in $A$, and $\mathcal{P}_{k}$ is $/ / \forall x A / / s$. By assumption, $\Gamma_{\mathrm{i}} \models_{\text {Fn }}^{*} / / A^{x / a} / / s$; but by the nature of access, $\Gamma_{i} \subseteq \Gamma_{k} \cup\left\{E a_{s}\right\}$; so by L9.I, $\Gamma_{k} \cup\left\{E_{s}\right\} \models_{\text {ma }}^{*} / / A^{x / a} / / s$. Suppose $\Gamma_{k} \not \nvdash_{\text {ma }}^{*} / / \forall x A / / s$; then by $\mathrm{VF}_{\mathrm{N}} \alpha^{*}$, there is an $F_{n} \alpha$ interpretation $\mathrm{I}=\langle\mathrm{W}, \mathrm{U}, \mathrm{D}, \mathrm{R}, \mathrm{P}, v\rangle_{\mathrm{m}}$ such that $h_{m}\left(\Gamma_{k}\right)=1$ but $h_{m(s)}(/ / \forall x A / /)=0$; from the latter, there is some $\delta$ such that $h_{m(s)}(/ / \forall x A / /) / / \delta=0$; so by $\operatorname{HF}(\forall)$, there is some $u \in D(m(s))$ such that $h_{m(s)}(/ / A / /) / / \delta[x \mid u]=0$. Let $I^{\prime}=\left\langle W, U, D, R, P, v^{\prime}\right\rangle_{m}$ be like I except that $v^{\prime}(\mathrm{a})=u$. Then since a does not occur in $\Gamma_{k}$, by the corollary to L9.3, it remains that $h_{m}^{\prime}\left(\Gamma_{k}\right)=1$; and since $u \in D(m(s))$, and $v^{\prime}(a)=u, v^{\prime}(a) \in D(m(s))$; so for arbitrary $\delta^{\prime}, \Delta^{\prime}(a) \in D(m(s))$; so $\Delta^{\prime}(\mathrm{a}) \in v_{m(s)}^{\prime}(\mathrm{E})$; so by $\mathrm{HF}(\mathrm{R}), \mathrm{h}_{\mathrm{m}(\mathrm{s})}^{\prime}(\mathrm{Ea}) / / \delta^{\prime}=1$; and since $\delta^{\prime}$ is arbitrary, $h_{m(s)}^{\prime}(E a)=1$; so $h_{m}^{\prime}\left(\Gamma_{k} \cup\left\{E_{s}\right\}\right)=1$; so by $V_{F N} \alpha^{*}$, $h_{m(s)}^{\prime}\left(/ / A^{x} / a / /\right)=1$; so $h_{m(s)}^{\prime}\left(/ / A^{x} / a / /\right) / / \delta=1$; but $\Delta(a)=v^{\prime}(a)=u$; so by L9.4, $h_{m(s)}^{\prime}(/ / A / /) / / \delta[x \mid u]=1$; so, since a does not occur in $A$, by L9.3, $h_{m(s)}(/ / A / /) / / \delta[x \mid u]=1$. This is impossible; reject the assumption: $\Gamma_{\mathrm{k}} \models_{\text {Tn } \alpha}^{*} / / \forall x A / / s$, which is to say, $\Gamma_{\mathrm{k}} \models_{\text {Tn } \alpha}^{*} \mathcal{P}_{\mathrm{k}}$.

$(\forall \mathrm{E})$ If $\mathcal{P}_{k}$ arises by $\forall \mathrm{E}$, then the picture is like this,

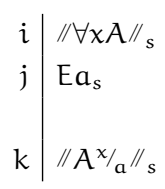

where $i, j<k$ and $\mathcal{P}_{k}$ is $/ / A^{x / a} / / s$. By assumption, $\Gamma_{i} \models_{\text {nn } \alpha}^{*} / / \forall x A / / s$ and $\Gamma_{\mathrm{j}} \models_{\text {nn } \alpha}^{*} E a_{s}$; but by the nature of access, $\Gamma_{\mathrm{i}} \subseteq \Gamma_{\mathrm{k}}$ and $\Gamma_{\mathrm{j}} \subseteq \Gamma_{\mathrm{k}}$; so

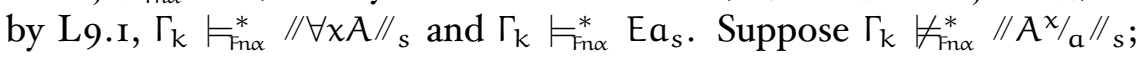
then by $\mathrm{VF}_{\mathrm{N}} \alpha^{*}$, there is an $F n \alpha$ interpretation $\langle\mathrm{W}, \mathrm{U}, \mathrm{D}, \mathrm{R}, \mathrm{P}, v\rangle_{\mathrm{m}}$ such that $h_{m}\left(\Gamma_{k}\right)=1$ but $h_{m(s)}\left(/ / A^{x / a} / /\right)=0$. From the latter, there is a $\delta$ such that $h_{m(s)}\left(/ / A^{x} /{ }^{\alpha} / /\right) / / \delta=0$. With the former, by $\operatorname{VF}_{N} \alpha^{*}$, $h_{m(s)}(/ / \forall x A / /)=1$ and $h_{m(s)}(E a)=1$; from the second of these, $h_{m(s)}(E a) / / \delta=1$; so by $\operatorname{HF}(R), \Delta(a) \in v_{m(s)}(E)$; so $\Delta(a) \in D(m(s))$; say $\Delta(a)=u$; then $u \in D(m(s))$. Since $h_{m(s)}(/ / \forall x A / /)=1$, we have $h_{m(s)}(/ / \forall x A / /) / / \delta=1$; so by $\operatorname{HF}(\forall)$, for any $v \in D(m(s)), h_{m(s)}(/ / A / /) / /$ $\delta[x \mid v]=1$; it follows that $h_{m(s)}(/ / A / /) / / \delta[x \mid u]=1$; so since $\Delta(a)=u$, by L9.4, $h_{m(s)}\left(/ / A^{x} / a^{/ /}\right) / / \delta=1$. This is impossible; reject the assumption: $\Gamma_{k} \models_{\text {ina }}^{*} / / A^{x / a} / / s$, which is to say, $\Gamma_{k} \models_{\text {ma }}^{*} \mathcal{P}_{k}$.

( $\square \mathrm{I})$ If $\mathcal{P}_{k}$ arises by $\square \mathrm{I}$, then the picture is like this,



where $i<k$, $t$ does not appear in any member of $\Gamma_{k}$ (in any undischarged 
premise or assumption), and $\mathcal{P}_{k}$ is $/ \square A / s$. By assumption, $\Gamma_{i} \models_{\text {fro }}^{*} / A / t$; but by the nature of access, $\Gamma_{i} \subseteq \Gamma_{k} \cup\{$ s.t $\}$; so by L9. I, $\Gamma_{k} \cup\{$ s.t $\} \models_{\text {m } \alpha}^{*} / A / t$. Suppose $\Gamma_{\mathrm{k}} \forall_{\text {m } \alpha}^{*} / \square \mathrm{A} / \mathrm{s}$; then by VFN $\alpha^{*}$, there is an $F n \alpha$ interpretation $\langle\mathrm{W}, \mathrm{U}, \mathrm{D}, \mathrm{R}, \mathrm{P}, v\rangle_{\mathrm{m}}$ such that $h_{\mathrm{m}}\left(\Gamma_{\mathrm{k}}\right)=1 \mathrm{but} \mathrm{h}_{\mathrm{m}(\mathrm{s})}(/ \square \mathrm{A} /)=0$; from the latter, there is some $\delta$ such that $h_{m(s)}(/ \square A /) / / \delta=0$; so by $H F(\square)$, there is some $w \in W$ such that $m(s) R w$ and $h_{w}(/ A /) / / \delta=0$. Now consider a map $m^{\prime}$ like $m$ except that $m^{\prime}(t)=w$, and consider $\langle W, U, D, R, P, v\rangle_{m^{\prime}}$; since $t$ does not appear in $\Gamma_{k}$, it remains that $h_{m^{\prime}}\left(\Gamma_{k}\right)=1$; and since $\mathrm{m}^{\prime}(\mathrm{t})=w$ and $\mathrm{m}^{\prime}(\mathrm{s})=\mathrm{m}(\mathrm{s}),\left\langle\mathrm{m}^{\prime}(\mathrm{s}), \mathrm{m}^{\prime}(\mathrm{t})\right\rangle \in \mathrm{R} ;$ so $\mathrm{h}_{\mathrm{m}^{\prime}}\left(\Gamma_{\mathrm{k}} \cup\{\right.$ s.t $\left.\}\right)=1$;

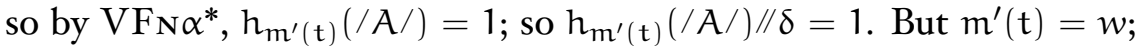
so $h_{w}(/ A /) / / \delta=1$. This is impossible; reject the assumption: $\Gamma_{k} \models_{\text {ma }}^{*}$ $/ \square \mathrm{A} / \mathrm{s}$, which is to say, $\Gamma_{\mathrm{k}} \models_{\text {Fn } \alpha}^{*} \mathcal{P}_{\mathrm{k}}$.

( $\square \mathrm{E})$ If $\mathcal{P}_{k}$ arises by $\square \mathrm{E}$, then the picture is like this,

$$
\begin{array}{l|l}
\mathfrak{i} & / \square \mathrm{A} / \mathrm{s} \\
\mathfrak{j} & \mathrm{s.t} \\
\mathrm{k} & / \mathrm{A} / \mathrm{t}
\end{array}
$$

where $i, j<k$ and $\mathcal{P}_{k}$ is $/ A / t$. By assumption, $\Gamma_{i} \models_{\text {Tn } \alpha}^{*} / \square A / s$ and $\Gamma_{j} \models_{\text {tn } \alpha}^{*}$ s.t; but by the nature of access, $\Gamma_{\mathrm{i}} \subseteq \Gamma_{\mathrm{k}}$ and $\Gamma_{\mathrm{j}} \subseteq \Gamma_{\mathrm{k}}$; so by L9.I, $\Gamma_{\mathrm{k}} \models_{\text {Fn }}^{*}$ $/ \square \mathrm{A} / \mathrm{s}$ and $\Gamma_{\mathrm{k}} \models_{\text {m } \alpha}^{*}$ s.t. Suppose $\Gamma_{\mathrm{k}} \forall_{\text {m } \alpha}^{*} / \mathrm{A} / \mathrm{t}$; then by $\mathrm{VFN}_{\mathrm{N}} \alpha^{*}$, there is an $F n \alpha$ interpretation $\langle W, U, D, R, P, v\rangle_{m}$ such that $h_{m}\left(\Gamma_{k}\right)=1$ but $h_{m(t)}(/ A /)=0$. From the latter, there is a $\delta$ such that $h_{m(t)}(/ A /) / / \delta=$ 0 . With the former, by $\operatorname{VF}_{N} \alpha^{*}, h_{m(s)}(/ \square A /)=1$ and $\langle m(s), m(t)\rangle \in R$; from the first of these, $h_{m(s)}(/ \square A /) / / \delta=1$; so by $\operatorname{HF}(\square)$, for any $w \in$ W such that $m(s) R w, h_{w}(/ A /) / / \delta=1$; so $h_{m(t)}(/ A /) / / \delta=1$. This is

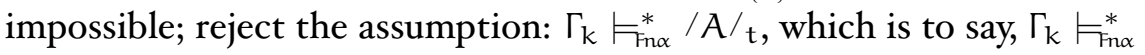
$\mathcal{P}_{k}$.

(D) If $\mathcal{P}_{k}$ arises by $\mathrm{D}$, then the picture is like this,

$$
\begin{array}{l|l}
i & A_{s} \\
k & \bar{A}_{s}
\end{array}
$$

where $i<k$ and $\mathcal{P}_{k}$ is $\bar{A}_{s}$. By assumption, $\Gamma_{i} \models_{\text {Tnd }}^{*} A_{s}$; but by the nature of access, $\Gamma_{\mathrm{i}} \subseteq \Gamma_{\mathrm{k}}$; so by L9.I, $\Gamma_{\mathrm{k}} \models_{\text {m } \alpha}^{*} A_{s}$. Suppose $\Gamma_{\mathrm{k}} \forall_{\text {in } \alpha}^{*} \bar{A}_{s}$; then by $\mathrm{VF}_{\mathrm{N}} \alpha^{*}$, there is an $F n \alpha$ interpretation $\langle\mathrm{W}, \mathrm{U}, \mathrm{D}, \mathrm{R}, \mathrm{P}, v\rangle_{\mathrm{m}}$ such that $h_{m}\left(\Gamma_{k}\right)=1$ but $h_{m(s)}(\bar{A})=0$. From the latter, there is a $\delta$ such that $h_{m(s)}(\bar{A}) / / \delta=0$. With the former, by $\operatorname{VFN}_{N} \alpha^{*}, h_{m(s)}(A)=1$; so $h_{m(s)}(A) / / \delta=1$. But for these interpretations, there is no formula $A$ and $w \in W$ such that $h_{w}(A) / / \delta=1$ and $h_{w}(\bar{A}) / / \delta=0$.

Basis: Suppose $A$ is an atomic $Q^{n^{n}} t_{1} \ldots t_{n}$, and for some $w \in W$ and $\delta, h_{w}(A) / / \delta=1$; then $h_{w}\left(Q^{n} t_{1} \ldots t_{n}\right) / / \delta=1$; so by $H F(R)$, 
$\left\langle\Delta\left(t_{1}\right) \ldots \Delta\left(t_{n}\right)\right\rangle \in v_{w}\left(Q^{n}\right) ;$ so by the construction of $\bar{v},\left\langle\Delta\left(t_{1}\right)\right.$ $\left.\ldots \Delta\left(t_{n}\right)\right\rangle \in \bar{v}_{w}\left(Q^{n}\right)$; so by $\mathrm{HF}(\mathrm{R}), h_{w}\left(\overline{\mathrm{Q}^{n} t_{1} \ldots t_{n}}\right) / / \delta=1$; so $h_{w}(\bar{A}) / / \delta=1$.

Assp: For any $i, 0 \leqslant i<k$, if $A$ has $i$ operators, and for some $w \in W$ and $\delta, h_{w}(A) / / \delta=1$, then $h_{w}(\bar{A}) / / \delta=1$.

Show: If $A$ has $k$ operators, and for some $w \in W$ and $\delta, h_{w}(A) / / \delta=1$, then $h_{w}(\bar{A}) / / \delta=1$.

If $A$ has $k$ operators, then $A$ is of the form, $\neg P, P \wedge Q, \square P$, or $\forall x P$, where $\mathrm{P}$ and $\mathrm{Q}$ have $<k$ operators.

$(\neg)$ Suppose $A$ is $\neg P$ and for some $w \in W$ and $\delta, h_{w}(A) / / \delta=1$; then $h_{w}(\neg \mathrm{P}) / / \delta=1$; so by $\mathrm{HF}(\neg), h_{w}(\overline{\mathrm{P}}) / / \delta=0$; so by assumption, $h_{w}(\mathrm{P}) / / \delta=0$; so by $\mathrm{HF}(\neg), h_{w}(\overline{\neg P}) / / \delta=1$; so $h_{w}(\bar{A}) / / \delta=1$.

$(\wedge)$ Suppose $A$ is $P \wedge Q$ and for some $w \in W$ and $\delta, h_{w}(A) / / \delta=$ 1 ; then $h_{w}(P \wedge Q) / / \delta=1$; so by $\operatorname{HF}(\wedge), h_{w}(P) / / \delta=1$ and $h_{w}(\mathrm{Q}) / / \delta=1$; so by assumption, $h_{w}(\bar{P}) / / \delta=1$ and $h_{w}(\bar{Q}) / / \delta=$ 1 ; so by $\operatorname{HF}(\wedge), h_{w}(\overline{\mathrm{P} \wedge \mathrm{Q}}) / / \delta=1$; so $h_{w}(\bar{A}) / / \delta=1$.

( $\square$ ) Suppose $A$ is $\square P$ and for some $w \in W$ and $\delta, h_{w}(A) / / \delta=1$ but $h_{w}(\bar{A}) / / \delta=0$; then $h_{w}(\square \mathrm{P}) / / \delta=1$ but $h_{w}(\overline{\square P}) / / \delta=0$; from the latter, by $\operatorname{HF}(\square)$, there is an $a \in W$ such that $w \operatorname{Ra}$ and $h_{a}(\bar{P}) / / \delta=0$; so by assumption, $h_{a}(P) / / \delta=0$; so by $H F(\square)$, $h_{w}(\square P) / / \delta=0$. This is impossible; reject the assumption: if $h_{w}(A) / / \delta=1$ then $h_{w}(\bar{A}) / / \delta=1$.

$(\forall)$ Suppose $A$ is $\forall x P$ and for some $w \in W$ and $\delta, h_{w}(A) / / \delta=1$ but $h_{w}(\bar{A}) / / \delta=0$; then $h_{w}(\forall x P) / / \delta=1$ but $h_{w}(\overline{\forall x P}) / / \delta=0$; from the latter, by $\operatorname{HF}(\forall)$, there is some $u \in D(w)$ such that $h_{w}(\bar{P}) / / \delta[x \mid u]=0$; so by assumption, $h_{w}(\mathrm{P}) / / \delta[x \mid u]=0$; so by $\mathrm{HF}(\forall), h_{w}(\forall x \mathrm{P}) / / \delta=0$. This is impossible; reject the assumption: if $h_{w}(A) / / \delta=1$ then $h_{w}(\bar{A}) / / \delta=1$.

For any $A$, if for some $w \in W$ and $\delta, h_{w}(A) / / \delta=1$, then $h_{w}(\bar{A}) / / \delta=1$.

So, returning to the main case, $h_{m(s)}(\bar{A}) / / \delta=1$. This is impossible; reject the assumption: $\Gamma_{k} \models_{\text {nn }}^{*} \bar{A}_{s}$, which is to say, $\Gamma_{k} \models_{\text {nn }}^{*} \mathcal{P}_{k}$.

(=I) If $\mathcal{P}_{k}$ arises by $=\mathrm{I}$, then the picture is like this,

$$
\begin{array}{l|l}
i & P 1[a=a]_{s} \\
k & (a=a)_{s}
\end{array}
$$

where $i<k$ and $\mathcal{P}_{k}$ is $(a=a)_{s}$. By assumption, $\Gamma_{i} \models_{\text {nn }}^{*} P 1[a=a]_{s}$; but by the nature of access, $\Gamma_{i} \subseteq \Gamma_{k}$; so by L9.I, $\Gamma_{k} \models_{\text {Tn } \alpha}^{*} \mathrm{P} 1[a=a]_{s}$. Suppose $\Gamma_{k} \nvdash_{\text {in } \alpha}^{*}(a=a)_{s}$; then by $\operatorname{VFN}_{N} \alpha^{*}$, there is an $F n \alpha$ interpretation $\langle W, U, D, R, P, v\rangle_{m}$ such that $h_{m}\left(\Gamma_{k}\right)=1$ but $h_{m(s)}(a=a)=0$. 
From the latter, there is a $\delta$ such that $h_{m(s)}(a=a) / / \delta=0$; so by $H F(R)$, $\langle\Delta(\mathrm{a}), \Delta(\mathrm{a})\rangle \notin v_{\mathrm{m}(\mathrm{s})}(=)$. Now $\mathrm{P}(=)$ is $\langle 0,0\rangle$ or not; if $\mathrm{P}(=)=\langle 0,0\rangle$, then $v_{\mathrm{m}(\mathrm{s})}(=)=\{\langle\mathfrak{u}, \mathfrak{u}\rangle \mid \mathfrak{u} \in \mathbb{U}\}$; so $\langle\Delta(\mathfrak{a}), \Delta(\mathfrak{a})\rangle \in v_{\mathrm{m}(\mathrm{s})}(=)$; this is impossible, so $\mathrm{P}(=) \neq\langle 0,0\rangle$, and $v_{\mathrm{m}(\mathrm{s})}(=)=\{\langle\mathrm{u}, \mathrm{u}\rangle \mid \mathrm{u} \in \mathrm{D}(\mathrm{m}(\mathrm{s}))\}$. But since $h_{m}\left(\Gamma_{k}\right)=1$, by VFN $\alpha^{*}, h_{m(s)}(P 1[a=a])=1$; so $h_{m(s)}(P 1[a=$ a]) $/ / \delta=1$; so with $\operatorname{HF}(\wedge)$, for an $i$ such that $P(=)_{i} \geqslant 1, h_{m(s)}(E a) / / \delta=$ 1 , and by $\operatorname{HF}(\mathrm{R}), \Delta(\mathrm{a}) \in v_{w(s)}(\mathrm{E})$, so that $\Delta(\mathrm{a}) \in \mathrm{D}(\mathrm{m}(\mathrm{s}))$; and since there is some such $i, \Delta(a) \in D(m(s))$; so $\langle\Delta(a), \Delta(a)\rangle \in v_{m(s)}(=)$. This is impossible; reject the assumption: $\Gamma_{k} \models_{\text {tna }}^{*}(a=a)_{s}$, which is to say, $\Gamma_{\mathrm{k}} \models_{\text {Tn } \alpha}^{*} \mathcal{P}_{\mathrm{k}}$.

(=E) If $A_{k}$ arises by $=E$, then the picture is like this,

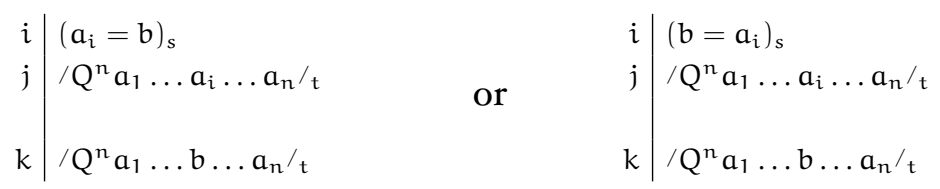

where $i, j<k$ and $\mathcal{P}_{k}$ is $/ Q^{n} a_{1} \ldots b \ldots a_{n} / t$. In the first case, by assumption, $\Gamma_{i} \models_{\text {ma }}^{*}\left(a_{i}=b\right)_{s}$ and $\Gamma_{j} \models_{\text {Tn } \alpha}^{*} / Q^{n} a_{1} \ldots a_{i} \ldots a_{n} / t$; but by the nature of access, $\Gamma_{i} \subseteq \Gamma_{k}$ and $\Gamma_{j} \subseteq \Gamma_{k}$; so by L9.I, $\Gamma_{k} \models_{\text {Fn } \alpha}^{*}\left(a_{i}=b\right)_{s}$

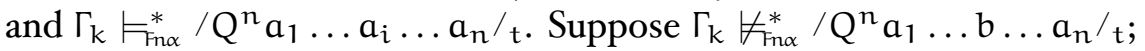
then by $\mathrm{VFN}_{\mathrm{N}} \alpha^{*}$, there is an $F n \alpha$ interpretation $\langle\mathrm{W}, \mathrm{U}, \mathrm{D}, \mathrm{R}, \mathrm{P}, v\rangle_{\mathrm{m}}$ such that $h_{m}\left(\Gamma_{k}\right)=1$ but $h_{m(t)}\left(/ Q^{n} a_{1} \ldots b \ldots a_{n} /\right)=0$. From the latter, there is a $\delta$ such that $h_{m(t)}\left(/ Q^{n} a_{1} \ldots b \ldots a_{n} /\right) / / \delta=0$; so by $\operatorname{HF}(R)$, $\left\langle\Delta\left(a_{1}\right) \ldots \Delta(b) \ldots \Delta\left(a_{n}\right)\right\rangle \notin / \nu /{ }_{m(t)}\left(Q^{n}\right)$. But since $h_{m}\left(\Gamma_{k}\right)=1$, by $V_{F} \alpha^{*}, h_{m(s)}\left(a_{i}=b\right)=1$ and $h_{m(t)}\left(/ Q^{n} a_{1} \ldots a_{i} \ldots a_{n} /\right)=1$; so $h_{m(s)}\left(a_{i}=b\right) / / \delta=1$ and $h_{m(t)}\left(/ Q^{n} a_{1} \ldots a_{i} \ldots a_{n} /\right) / / \delta=1$. From the first of these, by $\operatorname{HF}(\mathrm{R}),\left\langle\Delta\left(a_{i}\right), \Delta(b)\right\rangle \in v_{m(s)}(=)$; so, on either specification of $v_{m(s)}(=), \Delta\left(a_{i}\right)=\Delta(b)$. From the second, by $H F(R)$, $\left\langle\Delta\left(a_{1}\right) \ldots \Delta\left(a_{i}\right) \ldots \Delta\left(a_{n}\right)\right\rangle \in / \nu / m(t)\left(Q^{n}\right) ;$ so $\left\langle\Delta\left(a_{1}\right) \ldots \Delta(b) \ldots \Delta\left(a_{n}\right)\right\rangle$ $\in / \nu / m(t)\left(Q^{n}\right)$. This is impossible; reject the assumption: $\Gamma_{k} \models_{\text {Tn } \alpha}^{*}$ $/ Q^{n} a_{1} \ldots b \ldots a_{n} / t$, which is to say, $\Gamma_{k} \models_{\text {nn }}^{*} \mathcal{P}_{k}$. And similarly in the other case.

(PII) If $\mathcal{P}_{k}$ arises by $P_{I} I$, then the picture is like this,

$$
\begin{array}{l|l}
i & \left(Q^{n} a_{1} \ldots a_{n}\right)_{s} \\
k & P 1\left[Q^{n} a_{1} \ldots a_{n}\right]_{s}
\end{array}
$$

where $i<k$ and $\mathcal{P}_{k}$ is $P 1\left[Q^{n} a_{1} \ldots a_{n}\right]_{s}$. By our assumption, $\Gamma_{i} \models_{\text {Tn }}^{*}$ $\left(Q^{n} a_{1} \ldots a_{n}\right)_{s}$; but by the nature of access, $\Gamma_{i} \subseteq \Gamma_{k}$; so by L9.I, we

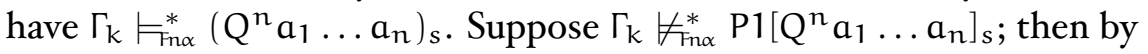
$\mathrm{VF}_{N} \alpha^{*}$, there is some $F n \alpha$ interpretation $\langle\mathrm{W}, \mathrm{U}, \mathrm{D}, \mathrm{R}, \mathrm{P}, v\rangle_{\mathrm{m}}$ such that $h_{m}\left(\Gamma_{k}\right)=1$ but in which $h_{m(s)}\left(P 1\left[Q^{n} a_{1} \ldots a_{n}\right]\right)=0$. From the latter, there is a $\delta$ such that $h_{m(s)}\left(P 1\left[Q^{n} a_{1} \ldots a_{n}\right]\right) / / \delta=0$; so, with $\operatorname{HF}(\wedge)$, 
there is some $i$ such that $\mathrm{P}\left(\mathrm{Q}^{\mathfrak{n}}\right)_{i} \geqslant 1$ and $h_{\mathrm{m}(\mathrm{s})}\left(E a_{i}\right) / / \delta=0$; so by $\mathrm{HF}(\mathrm{R}), \Delta\left(\mathrm{a}_{\mathrm{i}}\right) \notin v_{\mathrm{m}(\mathrm{s})}(\mathrm{E})$; so $\Delta\left(\mathrm{a}_{\mathrm{i}}\right) \notin \mathrm{D}(\mathrm{m}(\mathrm{s}))$. But since $h_{\mathrm{m}}\left(\Gamma_{\mathrm{k}}\right)=1$, by VFN $\alpha^{*}, h_{m(s)}\left(Q^{n} a_{1} \ldots a_{n}\right)=1$; so $h_{m(s)}\left(Q^{n} a_{1} \ldots a_{n}\right) / / \delta=1$; so by $\operatorname{HF}(\mathrm{R}),\left\langle\Delta\left(a_{1}\right) \ldots \Delta\left(a_{n}\right)\right\rangle \in v_{m(s)}\left(Q^{n} a_{1} \ldots a_{n}\right)$; so by EP, for $i$ with $\mathrm{P}\left(\mathrm{Q}^{\mathfrak{n}}\right)_{i} \geqslant 1$ as above, $\Delta\left(\mathrm{a}_{i}\right) \in \mathrm{D}(\mathrm{m}(\mathrm{s}))$. This is impossible; reject the

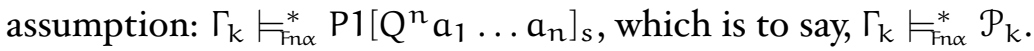

$\left(\mathrm{P}_{2} \mathrm{I}\right)$ If $\mathcal{P}_{k}$ arises by $\mathrm{P}_{2} \mathrm{I}$, then the picture is like this,

\begin{tabular}{l|l}
$i$ & $\left(\neg Q^{n} a_{1} \ldots a_{n}\right)_{s}$ \\
$k$ & $P 2\left[Q^{n} a_{1} \ldots a_{n}\right]_{s}$
\end{tabular}

where $i<k$ and $\mathcal{P}_{k}$ is $P 2\left[Q^{n} a_{1} \ldots a_{n}\right]_{s}$. By assumption, we have $\Gamma_{i} \models_{\text {mnd }}^{*}$ $\left(\neg Q^{n} a_{1} \ldots a_{n}\right)_{s}$; but by the nature of access, $\Gamma_{i} \subseteq \Gamma_{k}$; so by L9.I, $\Gamma_{\mathrm{k}} \models_{\text {ind }}^{*}\left(\neg \mathrm{Q}^{\mathrm{n}} \mathrm{a}_{1} \ldots \mathrm{a}_{\mathrm{n}}\right)_{s}$. Suppose that $\Gamma_{\mathrm{k}} \forall_{\text {tnd }}^{*} \mathrm{P} 2\left[\mathrm{Q}^{\mathrm{n}} \mathrm{a}_{1} \ldots \mathrm{a}_{\mathrm{n}}\right]_{s}$; then by $\mathrm{VF}_{N} \alpha^{*}$, there is some $F n \alpha$ interpretation $\langle\mathrm{W}, \mathrm{U}, \mathrm{D}, \mathrm{R}, \mathrm{P}, v\rangle_{\mathrm{m}}$ such that $h_{m}\left(\Gamma_{k}\right)=1$ but in which $h_{m(s)}\left(P 2\left[Q^{n} a_{1} \ldots a_{n}\right]\right)=0$. From the latter, there is a $\delta$ such that $h_{m(s)}\left(P 2\left[Q^{n} a_{1} \ldots a_{n}\right]\right) / / \delta=0$; so, with $\mathrm{HF}(\wedge)$, there is some $i$ such that $\mathrm{P}\left(\mathrm{Q}^{\mathrm{n}}\right)_{i}=2$ and $h_{\mathrm{m}(\mathrm{s})}\left(\mathrm{Ea}_{i}\right) / / \delta=0$; so by $\operatorname{HF}(\mathrm{R}), \Delta\left(a_{i}\right) \notin v_{m(s)}(E)$; so $\Delta\left(a_{i}\right) \notin D(m(s))$. But since $h_{m}\left(\Gamma_{k}\right)=1$, by VFN $\alpha^{*}, h_{m(s)}\left(\neg Q^{n} a_{1} \ldots a_{n}\right)=1$; so $\left.h_{m(s)}\left(\neg Q^{n} a_{1} \ldots a_{n}\right)\right) / / \delta=1$; so by $\mathrm{HF}(\neg), h_{m(s)}\left(\overline{Q^{n} a_{1} \ldots a_{n}}\right)=0$; so by $\operatorname{HF}(R),\left\langle\Delta\left(a_{1}\right) \ldots \Delta\left(a_{n}\right)\right\rangle \notin$ $\bar{v}_{m(s)}\left(Q^{n} a_{1} \ldots a_{n}\right)$; so, by the construction of $\bar{v}$, for $i$ with $\mathrm{P}\left(\mathrm{Q}^{n}\right)_{i}=2$ as above, $\Delta\left(a_{i}\right) \in \mathrm{D}(\mathrm{m}(\mathrm{s}))$. This is impossible; reject the assumption:



$\left(\mathrm{P}_{2} \mathrm{E}\right)$ If $\mathcal{P}_{k}$ arises by $\mathrm{P}_{2} \mathrm{E}$, then the picture is like this,

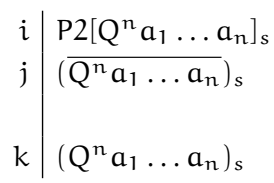

where $i, j<k$ and $\mathcal{P}_{k}$ is $\left(Q^{n} a_{1} \ldots a_{n}\right)_{s}$. By assumption, we have $\Gamma_{i} \models_{\text {Tnx }}^{*}$ $\mathrm{P} 2\left[\mathrm{Q}^{\mathrm{n}} \mathrm{a}_{1} \ldots \mathrm{a}_{n}\right]_{s}$ and $\Gamma_{j} \models_{\text {fn } \alpha}^{*}\left(\overline{\mathrm{Q}^{n} \mathrm{a}_{1} \ldots \mathrm{a}_{n}}\right)_{s}$; but by the nature of access,

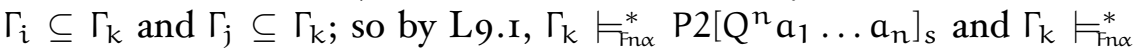
$\left(\overline{Q^{n} a_{1} \ldots a_{n}}\right)_{s}$. Suppose $\Gamma_{k} \nvdash_{\text {ind }}^{*}\left(Q^{n} a_{1} \ldots a_{n}\right)_{s}$; then by VFN $\alpha^{*}$, there is som Fn $\alpha$ interpretation $\langle W, U, D, R, P, v\rangle_{m}$ such that $h_{m}\left(\Gamma_{k}\right)=1$ but in which $h_{m(s)}\left(Q^{n} a_{1} \ldots a_{n}\right)=0$. From the latter, there is a $\delta$ such that $h_{m(s)}\left(Q^{n} a_{1} \ldots a_{n}\right) / / \delta=0$; so by $\operatorname{HF}(R)$, we have $\left\langle\Delta\left(a_{1}\right) \ldots \Delta\left(a_{n}\right)\right\rangle \notin$ $v_{m(s)}\left(Q^{n}\right)$. But since $h_{m}\left(\Gamma_{k}\right)=1$, by VFN $\alpha^{*}, h_{m(s)}\left(P 2\left[Q^{n} a_{1} \ldots a_{n}\right]\right)$ $=1$ and $h_{m(s)}\left(\overline{Q^{n} a_{1} \ldots a_{n}}\right)=1$; so $h_{m(s)}\left(P 2\left[Q^{n} a_{1} \ldots a_{n}\right]\right) / / \delta=1$ and $h_{m(s)}\left(\overline{Q^{n} a_{1} \ldots a_{n}}\right) / / \delta=1$; from the second of these, by $\operatorname{HF}(R),\left\langle\Delta\left(a_{1}\right)\right.$ $\left.\ldots \Delta\left(a_{n}\right)\right\rangle \in \bar{v}_{m(s)}\left(Q^{n}\right)$; so by the construction of $\bar{v}$, either $\left\langle\Delta\left(a_{1}\right) \ldots\right.$ $\left.\Delta\left(\mathrm{a}_{\mathrm{n}}\right)\right\rangle \in v_{\mathrm{m}(\mathrm{s})}\left(\mathrm{Q}^{\mathrm{n}}\right)$ or there is some $i$ such that $\mathrm{P}\left(\mathrm{Q}^{\mathrm{n}}\right)_{i}=2$ and $\Delta\left(a_{i}\right) \notin \mathrm{D}(\mathrm{m}(\mathrm{s}))$; so there is some $i$ such that $\mathrm{P}\left(\mathrm{Q}^{\mathfrak{n}}\right)_{i}=2$ and $\Delta\left(\mathrm{a}_{\mathrm{i}}\right) \notin$ 
$\mathrm{D}(\mathrm{m}(\mathrm{s}))$. But since $h_{m(s)}\left(P 2\left[\mathrm{Q}^{n} a_{1} \ldots a_{n}\right]\right) / / \delta=1$, with $\operatorname{HF}(\wedge)$, any such $i$ with $\mathrm{P}\left(\mathrm{Q}^{n}\right)_{i}=2$ has $h_{m(s)}\left(E a_{i}\right) / / \delta=1$; so by $\operatorname{HF}(\mathrm{R}), \Delta\left(a_{i}\right) \in$ $v_{m(s)}(E)$; so $\Delta\left(a_{i}\right) \in D(m(s))$. This is impossible; reject the assumption: $\Gamma_{k} \models_{\text {ma }}^{*}\left(Q^{n} a_{1} \ldots a_{n}\right)_{s}$, which is to say, $\Gamma_{k} \models_{\text {ma }}^{*} \mathcal{P}_{k}$.

$(\mathrm{AM \eta})$ If $\mathcal{P}_{k}$ arises by $\mathrm{AM \eta}$, then the picture is like this,

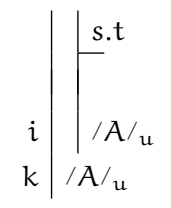

where $i<k$, $t$ does not appear in any member of $\Gamma_{k}$ (in any undischarged premise or assumption) and is not $u$, and $\mathcal{P}_{k}$ is $/ A / u$. Where this rule is included in NFn $\alpha, F n \alpha$ includes condition $\eta$. By assumption, $\Gamma_{\mathrm{i}} \models_{\text {in } \alpha}^{*} / \mathrm{A} / \mathrm{u}$; but by the nature of access, $\Gamma_{\mathrm{i}} \subseteq \Gamma_{\mathrm{k}} \cup\{$ s.t $\}$; so by L9.I, $\Gamma_{k} \cup\{$ s.t $\} \models_{\text {nn } \alpha}^{*} / A / u$. Suppose $\Gamma_{k} \not \forall_{\text {nn } \alpha}^{*} / A / u$; then by VFN $\alpha^{*}$, there is a $F n \alpha$ interpretation $\langle W, U, D, R, P, v\rangle_{m}$ such that $h_{m}\left(\Gamma_{k}\right)=1$ but $h_{m(u)}(/ A /)=0$. By condition $\eta$, there is a $w \in W$ such that $\mathrm{m}(\mathrm{s}) \mathrm{R} w$; consider a map $\mathrm{m}^{\prime}$ like $\mathrm{m}$ except that $\mathrm{m}^{\prime}(\mathrm{t})=w$, and consider $\langle W, U, D, R, P, v\rangle_{m^{\prime}}$; since $t$ does not appear in $\Gamma_{k}$, it remains that $h_{m^{\prime}}\left(\Gamma_{k}\right)=1$; and since $m^{\prime}(s)=m(s)$ and $m^{\prime}(t)=w,\left\langle m^{\prime}(s), m^{\prime}(t)\right\rangle \in R$; so $h_{m^{\prime}}\left(\Gamma_{k} \cup\{\right.$ s.t $\left.\}\right)=1$; so by VFN $\alpha^{*}, h_{m^{\prime}(u)}(/ A /)=1$. But since $t \neq u$, $m^{\prime}(u)=m(u)$; so $h_{m(u)}(/ A /)=1$. This is impossible; reject the as-

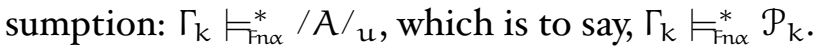

$(\mathrm{AM} \rho)$

$(\mathrm{AM} \sigma)$ If $\mathcal{P}_{k}$ arises by $\mathrm{AM} \sigma$, then the picture is like this,

$$
\begin{array}{l|l}
i & s . t \\
k & t . s
\end{array}
$$

where $i<k$ and $\mathcal{P}_{k}$ is t.s. Where this rule is in NFn $\alpha, F n \alpha$ includes condition $\sigma$. By assumption, $\Gamma_{i} \models_{\text {Tnd }}^{*}$ s.t; but by the nature of access,

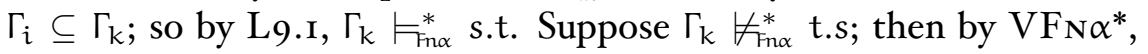
there is some $F n \alpha$ interpretation $\langle W, U, D, R, P, v\rangle_{m}$ such that $h_{m}\left(\Gamma_{k}\right)=$ 1 but $\langle m(t), m(s)\rangle \notin R$; since $h_{m}\left(\Gamma_{k}\right)=1$, by VFN $\alpha^{*},\langle m(s), m(t)\rangle \in R$; and by condition $\sigma$, for any $\langle x, y\rangle \in R,\langle y, x\rangle \in R$; so $\langle m(t), m(s)\rangle \in R$. This is impossible; reject the assumption: $\Gamma_{k} \models_{\text {Tn }}^{*}$ t.s, which is to say, $\Gamma_{\mathrm{k}} \models_{\text {tnd }}^{*} \mathcal{P}_{\mathrm{k}}$.

$(\mathrm{AM \tau})$

For any $i, \Gamma_{i} \models_{\text {Tn }}^{*} \mathcal{P}_{i}$. 
THEOREM 9.2 NFn $\alpha$ is complete: if $\Gamma \models_{\mathrm{n} n} / \mathrm{A} /$ then $\Gamma \vdash_{\mathrm{NFn} \alpha} / \mathrm{A} /$.

Suppose $\Gamma \models_{\text {in } \alpha} / A /$; then $\Gamma_{0} \models_{\text {fn } \alpha}^{*} / A / 0$; we show that $\Gamma_{0} \vdash_{N F n \alpha}^{*} / A / 0$. As usual, this reduces to the standard notion. For the following, fix on some particular $F n \alpha$. Then definitions of consistency etc. are relative to it.

Con $\Gamma$ is Consistent iff there is no $/ A / s$ such that $\Gamma \vdash_{\mathrm{NFn} \alpha}^{*} / A / s$ and $\Gamma \vdash_{\mathrm{N} n \alpha}^{*}$ $\backslash \neg A \backslash_{s}$.

L9.5 If $s$ is 0 or appears in $\Gamma$, and $\Gamma \nvdash_{N F n \alpha}^{*} \backslash \neg P \backslash_{s}$, then $\Gamma \cup\{/ \mathrm{P} / s\}$ is consistent.

Suppose $s$ is 0 or appears in $\Gamma$ and $\Gamma \nvdash_{N \text { r n } \alpha}^{*} \backslash \neg P \backslash_{s}$ but $\Gamma \cup\{/ \mathrm{P} / s\}$ is inconsistent. Then there is some $A_{t}$ such that $\Gamma \cup\{/ \mathrm{P} / s\} \vdash_{\mathrm{NFn} \alpha}^{*} / / \mathrm{A} / / \mathrm{t}$ and $\Gamma \cup\{/ \mathrm{P} / s\} \vdash_{\mathrm{NFn} \alpha}^{*}\|\neg A\|_{t}$. But then we can argue,

$$
\begin{array}{l|ll}
\mathrm{I} & \Gamma & \\
2 & \mid / \mathrm{P} / \mathrm{s} & \mathrm{A}(c, \neg \mathrm{I}) \\
& \mid / \mathrm{A} / / \mathrm{t} & \text { from } \Gamma \cup\{/ \mathrm{P} / \mathrm{s}\} \\
4 & \|\neg A\|_{\mathrm{t}} & \text { from } \Gamma \cup\{/ \mathrm{P} / \mathrm{s}\} \\
5 & \backslash \neg \mathrm{P} \backslash_{\mathrm{s}} & 2-4 \neg \mathrm{I}
\end{array}
$$

where the assumption is allowed insofar as $s$ is either 0 or appears in $\Gamma$; so $\Gamma \vdash_{\mathrm{NF} \alpha \alpha}^{*} \backslash \neg P \backslash_{s}$. But this is impossible; reject the assumption: if $s$ is 0 or appears in $\Gamma$ and $\Gamma \nvdash_{N F n \alpha}^{*} \backslash \neg P \backslash_{s}$, then $\Gamma \cup\{/ P / s\}$ is consistent.

LNG Let $\mathcal{L}$ be like our standard language except for the addition of constants $b_{1}, b_{2} \ldots$, and let $\Gamma_{0}^{\prime}$ be like $\Gamma_{0}$ except that its members are members of $\mathcal{L}$.

L9.6 For a derivation $D$ (or set $\Sigma$ ) say $D^{b / a}(\Sigma b / a)$ is like $D(\Sigma)$ except that each of its members has instances of $b$ replaced by $a$. Then if $D$ is $a$ derivation from $\Sigma$ and $a$ is a constant that does not appear in $D, D$ is a derivation from $\Sigma$ b/a.

L9.7 If $\Gamma_{0}$ is consistent, then $\Gamma_{0}^{\prime}$ is consistent.

Demonstrations for L9.6 and L9.7 on the model of parallel results for classical logic.

L9.8 There is an enumeration of all the subscripted sentences, $\mathcal{P}_{1} \mathcal{P}_{2} \ldots$

Proof by construction as usual.

Max $\Gamma$ is s-Maximal iff for any $A_{s}$ either $\Gamma \vdash_{\text {Nhn } \alpha}^{*} / A /_{s}$ or $\Gamma \vdash_{\text {NFn } \alpha}^{*} \backslash \neg A \backslash_{s}$.

SGT $\Gamma$ is a SCAPEGOAT set for $\square$ iff for every formula of the form $/ \neg \square A / s$, if $\Gamma \vdash_{\mathrm{NFn} \alpha}^{*} / \neg \square \mathrm{A} / \mathrm{s}$ then there is some $t$ such that $\Gamma \vdash_{\mathrm{N} F \mathrm{n} \alpha}^{*}$ s.t and $\Gamma \vdash_{\mathrm{N} F \mathrm{n} \alpha}^{*}$ $/ \neg \mathrm{A} / \mathrm{t}$.

$\Gamma$ is a SCAPEGOAT set for $\forall$ iff for every formula of the form $/ \neg \forall x \mathrm{~A} / \mathrm{s}$, if $\Gamma \vdash_{\mathrm{N} F n \alpha}^{*} / \neg \forall x \mathrm{~A} / \mathrm{s}$ then there is some a such that $\Gamma \vdash_{\mathrm{NFn \alpha}}^{*} \mathrm{Ea}_{\mathrm{s}}$ and $\Gamma \vdash_{\mathrm{N} F n \alpha}^{*}$ $/ \neg A^{x} / \mathrm{a} / s$. 
$\mathrm{C}\left(\Gamma^{\prime \prime}\right)$ For $\Gamma$ with unsubscripted sentences and the corresponding $\Gamma_{0}$ and $\Gamma_{0}^{\prime}$, we construct $\Gamma^{\prime \prime}$ as follows. Set $\Omega_{0}=\Gamma_{0}^{\prime}$. By L9.8, there is an enumeration, $\mathcal{P}_{1}, \mathcal{P}_{2} \ldots$ of all the subscripted sentences; let $\mathcal{E}_{0}$ be this enumeration. Then for the first $/ A / s$ in $\mathcal{E}_{i-1}$ such that $s$ is 0 or included in $\Omega_{i-1}$, let $\mathcal{E}_{i}$ be like $\varepsilon_{i-1}$ but without / $/$ /s, and set,

and

$$
\begin{array}{ll}
\Omega_{i}=\Omega_{i-1} & \text { if } \Omega_{i-1} \vdash_{N F n \alpha}^{*} \backslash \neg A \backslash_{s} \\
\Omega_{i^{*}}=\Omega_{i-1} \cup\{/ A / s\} & \text { if } \Omega_{i-1} \nvdash_{N F n \alpha}^{*} \backslash \neg A \backslash_{s}
\end{array}
$$

$$
\begin{aligned}
& \Omega_{i}=\Omega_{i^{*}} \quad \text { if } / A / s \text { is not of the form } / \neg \square \mathrm{P} / \mathrm{s} \text { or } \\
& \neg \forall \times \mathrm{PP} / \mathrm{s} \\
& \Omega_{i}=\Omega_{i^{*}} \cup\{\text { s.t, } / \neg P / t\} \quad \text { if } / A / s \text { is of the form } / \neg \square P / s \\
& \text {-where } t \text { is the first subscript not included in } \Omega_{i^{*}} \\
& \Omega_{i}=\Omega_{i^{*}} \cup\left\{\mathrm{Eb}_{s}, / \neg \mathrm{Px}^{\mathrm{b}} / \mathrm{s}\right\} \text { if } / \mathrm{A} / \mathrm{s} \text { is of the form } / \neg \forall \mathrm{XP} / \mathrm{s} \\
& \text {-where } b \text { is the first new constant not included in } \Omega_{i^{*}} \\
& \Gamma^{\prime \prime}=\bigcup_{i \geqslant 0} \Omega_{i}
\end{aligned}
$$

then

Note that there is always sure to be a subscript $t$ not in $\Omega_{i^{*}}$ insofar as there are infinitely many subscripts, and at any stage only finitely many formulas are added - the only subscripts in the initial $\Omega_{0}$ being 0 . And similarly, there is sure to be a constant $b$ not in $\Omega_{i^{*}}$ since the only constants in $\Omega_{0}$ are from the original language. Suppose s appears in $\Gamma^{\prime \prime}$; then there is some $\Omega_{i}$ in which it is first appears; and any formula $\mathcal{P}_{j}$ in the original enumeration that has subscript $s$ is sure to be "considered" for inclusion at a subsequent stage.

L9.9 For any s included in $\Gamma^{\prime \prime}$, $\Gamma^{\prime \prime}$ is s-maximal.

Suppose $s$ is included in $\Gamma^{\prime \prime}$ but $\Gamma^{\prime \prime}$ is not s-maximal. Then there is some $A_{s}$ such that $\Gamma^{\prime \prime} \nvdash_{\mathrm{N} n \alpha}^{*} / A /_{s}$ and $\Gamma^{\prime \prime} \nvdash_{\mathrm{N} \text { n } \alpha}^{*} \backslash \neg A \backslash_{s}$. For any $i$, each member of $\Omega_{i-1}$ is in $\Gamma^{\prime \prime}$; so if $\Omega_{i-1} \vdash_{\mathrm{NFn} \alpha}^{*} \backslash \neg A \backslash_{s}$ then $\Gamma^{\prime \prime} \vdash_{\mathrm{NFn} \alpha}^{*} \backslash \neg A \backslash_{s}$; but $\Gamma^{\prime \prime} \nvdash_{\mathrm{NFn} \alpha}^{*} \backslash \neg A \backslash_{s}$; so $\Omega_{i-1} \nvdash_{\mathrm{NFn} \alpha}^{*} \backslash \neg A \backslash_{s}$; so since $s$ is included in $\Gamma^{\prime \prime}$, there is a stage in the construction that sets $\Omega_{i^{*}}=\Omega_{i-1} \cup\{/ A / s\}$; so by construction, /A/s $\in \Gamma^{\prime \prime}$; so $\Gamma^{\prime \prime} \vdash_{\mathrm{NFn} \alpha}^{*} / A / s$. This is impossible; reject the assumption: $\Gamma^{\prime \prime}$ is s-maximal.

L9.Io If $\Gamma_{0}^{\prime}$ is consistent, then each $\Omega_{i}$ is consistent.

Suppose $\Gamma_{0}^{\prime}$ is consistent.

Basis: $\Omega_{0}=\Gamma_{0}^{\prime}$ and $\Gamma_{0}^{\prime}$ is consistent; so $\Omega_{0}$ is consistent.

Assp: For any $i, 0 \leqslant i<k, \Omega_{i}$ is consistent.

Show: $\Omega_{k}$ is consistent.

$\Omega_{k}$ is either (i) $\Omega_{k-1}$, (ii) $\Omega_{k^{*}}=\Omega_{k-1} \cup\{/ A / s\}$, (iii) $\Omega_{k^{*}} \cup$ $\{$ s.t, $/ \neg \mathrm{P} / \mathrm{t}\}$, or (iv) $\Omega_{\mathrm{k}^{*}} \cup\left\{\mathrm{Eb}_{\mathrm{s}}, / \neg \mathrm{P}^{\mathrm{x}} / \mathrm{b} / \mathrm{s}\right\}$. 
(i) Suppose $\Omega_{k}$ is $\Omega_{k-1}$. By assumption, $\Omega_{k-1}$ is consistent; so $\Omega_{k}$ is consistent.

(ii) Suppose $\Omega_{k}$ is $\Omega_{k^{*}}=\Omega_{k-1} \cup\{/ A / s\}$. Then by construction, $s$ is 0 or in $\Omega_{k-1}$ and $\Omega_{k-1} \nvdash_{\text {Nfrn }}^{*} \backslash \neg A \backslash_{s}$; so by L9.5, $\Omega_{k-1} \cup\{/ A / s\}$ is consistent; so $\Omega_{k}$ is consistent.

(iii) Suppose $\Omega_{\mathrm{k}}$ is $\Omega_{\mathrm{k}^{*}} \cup\{$ s.t, $/ \neg \mathrm{P} / \mathrm{t}\}$. In this case, as above, $\Omega_{\mathrm{k}^{*}}$ is consistent and by construction, $/ \neg \square \mathrm{P} / \mathrm{s} \in \Omega_{\mathrm{k}^{*}}$. Suppose that $\Omega_{k}$ is inconsistent. Then there is some $A_{\mathfrak{u}}$ such that $\Omega_{k^{*}} \cup$ $\{$ s.t, $/ \neg \mathrm{P} / \mathrm{t}\} \vdash_{\mathrm{NFn} \alpha}^{*} / / \mathrm{A} / / \mathrm{u}$ and $\Omega_{\mathrm{k}^{*}} \cup\{$ s.t, $/ \neg \mathrm{P} / \mathrm{t}\} \vdash_{\mathrm{NFn} \alpha}^{*}\|\neg A\|_{\mathrm{u}}$. In this case, reason as follows,

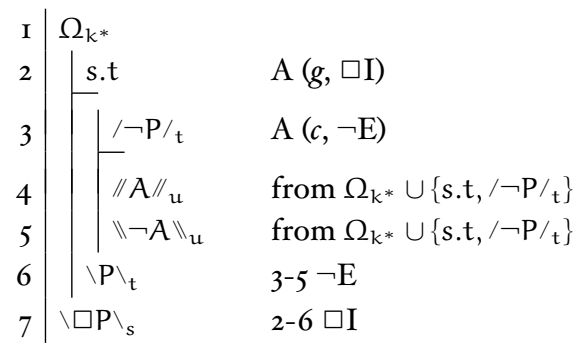

where, by construction, $\mathrm{t}$ is not in $\Omega_{\mathrm{k}^{*}}$. So $\Omega_{\mathrm{k}^{*}} \vdash_{\mathrm{NFn} \alpha}^{*} \backslash \square \mathrm{P} \backslash_{s}$; but $/ \neg \square \mathrm{P} / \mathrm{s} \in \Omega_{\mathrm{k}^{*}}$; so $\Omega_{\mathrm{k}^{*}} \vdash_{\mathrm{NFn} \alpha}^{*} / \neg \square \mathrm{P} / \mathrm{s}$; so $\Omega_{\mathrm{k}^{*}}$ is inconsistent. This is impossible; reject the assumption: $\Omega_{\mathrm{k}}$ is consistent.

(iv) Suppose $\Omega_{\mathrm{k}}$ is $\Omega_{\mathrm{k}^{*}} \cup\left\{\mathrm{Eb}_{\mathrm{s}}, / \mathrm{yP}^{\mathrm{x}} / \mathrm{b} / \mathrm{s}\right\}$. In this case, as above, $\Omega_{\mathrm{k}^{*}}$ is consistent and by construction, $/ \neg \forall x \mathrm{P} / \mathrm{s} \in \Omega_{\mathrm{k}^{*}}$. Suppose $\Omega_{k}$ is inconsistent. Then there is some $A_{u}$ such that $\Omega_{k^{*}} \cup$ $\left\{\mathrm{Eb}_{\mathrm{s}}, / \mathrm{P}^{\mathrm{x}} / \mathrm{b} / \mathrm{s}\right\} \vdash_{\mathrm{NFn} \alpha}^{*} / / \mathrm{A} / / \mathrm{u}$ and $\Omega_{\mathrm{k}^{*}} \cup\left\{\mathrm{Eb}_{\mathrm{s}}, / \mathrm{P}^{\mathrm{x}} / \mathrm{b} / \mathrm{s}\right\} \vdash_{\mathrm{NFn} \alpha}^{*}\|\neg \mathrm{A}\|_{\mathrm{u}}$. So reason as follows,

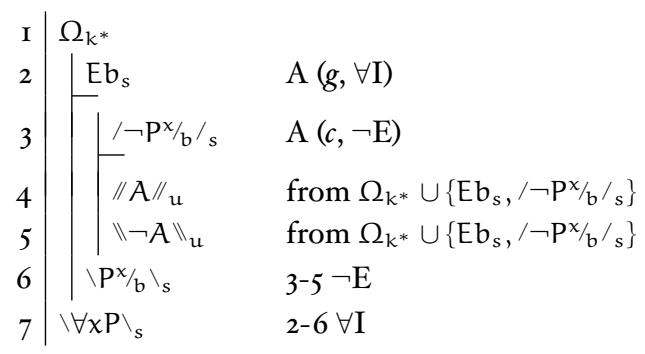

where, by construction, $b$ does not appear in $\Omega_{k^{*}}$ or in P. So $\Omega_{\mathrm{k}^{*}} \vdash_{\mathrm{N} \text { hn } \alpha}^{*} \backslash \forall \mathrm{xP} \backslash_{\mathrm{s}}$; but $/ \neg \forall \mathrm{xP} / \mathrm{s} \in \Omega_{\mathrm{k}^{*}} ;$ so $\Omega_{\mathrm{k}^{*}} \vdash_{\mathrm{NFn} \alpha}^{*} / \neg \forall \mathrm{xP} / \mathrm{s}$; so $\Omega_{\mathrm{K}^{*}}$ is inconsistent. This is impossible; reject the assumption: $\Omega_{\mathrm{k}}$ is consistent.

For any $i, \Omega_{i}$ is consistent.

L9.II If $\Gamma_{0}^{\prime}$ is consistent, then $\Gamma^{\prime \prime}$ is consistent.

Reasoning parallel to L2.6 and L6.6. 
L9.I2 If $\Gamma_{0}^{\prime}$ is consistent, then $\Gamma^{\prime \prime}$ is a scapegoat set for $\square$ and $\forall$.

For $(\square)$. Suppose $\Gamma_{0}^{\prime}$ is consistent and $\Gamma^{\prime \prime} \vdash_{\mathrm{NFn} \alpha}^{*} / \neg \square \mathrm{P} / \mathrm{s}$. By L9.II, $\Gamma^{\prime \prime}$ is consistent; and by the constraints on subscripts, $s$ is included in $\Gamma^{\prime \prime}$. Since $\Gamma^{\prime \prime}$ is consistent, $\Gamma^{\prime \prime} \nvdash_{\mathrm{N} \text { rn } \alpha}^{*} \backslash \neg \neg \square \mathrm{P} \backslash_{s}$; so there is a stage in the construction process where $\Omega_{i^{*}}=\Omega_{i-1} \cup\{/ \neg \square \mathrm{P} / \mathrm{s}\}$ and $\Omega_{i}=$ $\Omega_{i^{*}} \cup\{$ s.t, $/ \neg \mathrm{P} / \mathrm{t}\}$; so by construction, s.t $\in \Gamma^{\prime \prime}$ and $/ \neg \mathrm{P} / \mathrm{t} \in \Gamma^{\prime \prime}$; so $\Gamma^{\prime \prime} \vdash_{\mathrm{N} F n \alpha}^{*}$ s.t and $\Gamma^{\prime \prime} \vdash_{\mathrm{N} \text { hn } \alpha}^{*} / \neg \mathrm{P} / \mathrm{t}$. So $\Gamma^{\prime \prime}$ is a scapegoat set for $\square$.

For $\forall$. Suppose $\Gamma_{0}$ is consistent and $\Gamma^{\prime \prime} \vdash_{\text {NFn } \alpha}^{*} / \neg \forall x \mathrm{P} / \mathrm{s}$. By L9.II, $\Gamma^{\prime \prime}$ is consistent; and by the constraints on subscripts, $s$ is included in $\Gamma^{\prime \prime}$. Since $\Gamma^{\prime \prime}$ is consistent, $\Gamma^{\prime \prime} \nvdash_{\mathrm{NFn} \alpha}^{*} \backslash \neg \neg \forall x \mathrm{P} \backslash_{s}$; so there is a stage in the construction process where $\Omega_{i^{*}}=\Omega_{i-1} \cup\{/ \neg \forall x P / s\}$ and $\Omega_{i}=\Omega_{i^{*}} \cup$ $\left\{E b_{s}, / \neg \mathrm{P}^{\mathrm{x}} / \mathrm{b} / \mathrm{s}\right\}$; so by construction, $E b_{s} \in \Gamma^{\prime \prime}$ and $/ \neg \mathrm{P}^{\mathrm{x}} / \mathrm{b} / \mathrm{s} \in \Gamma^{\prime \prime}$; so $\Gamma^{\prime \prime} \vdash_{\mathrm{NFn} \alpha}^{*} E b_{s}$ and $\Gamma^{\prime \prime} \vdash_{\mathrm{N} F n \alpha}^{*} / \neg \mathrm{P}^{\mathrm{x}} / \mathrm{b} / \mathrm{s}$. So $\Gamma^{\prime \prime}$ is a scapegoat set for $\forall$.

$\mathrm{C}(\mathrm{I})$ We construct an interpretation $\mathrm{I}=\langle\mathrm{W}, \mathrm{U}, \mathrm{D}, \mathrm{R}, \mathrm{P}, v\rangle$ based on $\Gamma^{\prime \prime}$ as follows. Consider an enumeration $a_{1}, a_{2} \ldots$ of constants in $\mathcal{L}$ and say $i \simeq j$ iff $i=j$ or for some $s, \Gamma^{\prime \prime} \vdash_{\text {NFn } \alpha}^{*}\left(a_{i}=a_{j}\right)_{s}$, and set $\bar{i}=\{j \mid i \simeq j\}$. Then for $I=\langle W, U, D, R, P, v\rangle$, let $W$ have a member $w_{s}$ corresponding to each subscript $s$ included in $\Gamma^{\prime \prime}$. Set $U=\{\bar{i} \mid i \geqslant 1\}$, and $\bar{i} \in D\left(w_{s}\right)$ iff $\Gamma^{\prime \prime} \vdash_{\text {NFn } \alpha}^{*}\left(E a_{i}\right)_{s}$. And set $\left\langle w_{s}, w_{t}\right\rangle \in R$ iff $\Gamma^{\prime \prime} \vdash_{\text {NFn } \alpha}^{*}$ s.t. For any $a_{i}$, let $v\left(a_{i}\right)=\bar{i}$; and $\langle\bar{i} \ldots \bar{j}\rangle \in v_{w_{s}}\left(Q^{n}\right)$ iff $\Gamma^{\prime \prime} \vdash_{N \text { N } \alpha}^{*}\left(Q^{n} a_{1} \ldots a_{n}\right)_{s}$. Set $P$ directly from specification of the derivation system: if $E t_{i}$ is a conjunct of an arbitrary $\mathrm{P} 2\left[\mathrm{Q}^{\mathrm{n}} \mathrm{t}_{1} \ldots \mathrm{t}_{\mathrm{n}}\right]_{s}$, then $\mathrm{P}\left(\mathrm{Q}^{\mathrm{n}}\right)_{i}=2$; if $E t_{i}$ is a conjunct of an arbitrary $\mathrm{P} 1\left[\mathrm{Q}^{\mathrm{n}} \mathrm{t}_{1} \ldots \mathrm{t}_{n}\right]_{s}$ but not $\mathrm{P} 2\left[\mathrm{Q}^{\mathrm{n}} \mathrm{t}_{1} \ldots \mathrm{t}_{n}\right]_{s}$, then $\mathrm{P}\left(\mathrm{Q}^{\mathrm{n}}\right)_{i}=1$; otherwise $\mathrm{P}\left(\mathrm{Q}^{\mathrm{n}}\right)_{i}=0$.

Note that, for arbitrary $\delta, \Delta\left(a_{k}\right)=v\left(a_{k}\right)=\bar{k}$.

L.I3 I $=\langle W, U, D, R, P, v\rangle$ constructed as above is consistently specified.

reflexivity: For any $i, i \simeq i$. By construction.

symmetry: For any $i$ and $j$, if $i \simeq j$, then $j \simeq i$. Suppose $i \simeq j$. If $i=$ $j$, the result is immediate. So suppose $i \neq j$; then for some $s$, $\Gamma^{\prime \prime} \vdash_{\mathrm{NFn} \alpha}^{*}\left(a_{i}=a_{j}\right)_{s}$; so by $=E, \Gamma^{\prime \prime} \vdash_{\text {NFn } \alpha}^{*}\left(a_{i}=a_{i}\right)_{s}$; and by $=E$ again, $\Gamma^{\prime \prime} \vdash_{\text {N }}^{*} \alpha\left(a_{j}=a_{i}\right)_{s} ;$ so $j \simeq i$.

transitivity: For any $i, j$, and $k$, if $i \simeq j$, and $j \simeq k$, then $i \simeq k$. Suppose $i \simeq j$ and $j \simeq k$. If $i=j$ or $j=k$, the result is immediate. So suppose $i \neq j$ and $j \neq k$; then for some $s$ and $t, \Gamma^{\prime \prime} \vdash_{\text {NFn } \alpha}^{*}\left(a_{i}=a_{j}\right)_{s}$ and $\Gamma^{\prime \prime} \vdash_{\mathrm{N} \text { n } \alpha}^{*}\left(a_{j}=a_{k}\right)_{t}$; so by $=E, \Gamma^{\prime \prime} \vdash_{\text {NFn }}^{*}\left(a_{i}=a_{k}\right)_{s}$; so $i \simeq k$.

self-membership: For any $i, i \in \bar{i}$. For any $i$, by reflexivity, $i \simeq i$; so $i \in \bar{i}$.

uniqueness: For any $i$, if $i \in \bar{h}$ and $i \in \bar{k}$, then $\bar{h}=\bar{k}$. Suppose there are $h$ and $k$ such that $i \in \bar{h}$ and $i \in \bar{k}$, but $h \neq k$. From the latter, there is some $j$ such that $j \in \bar{h}$ and $j \notin \bar{k}$, or $j \in \bar{k}$ and $j \notin \bar{h}$; without loss of generality, suppose the former; then $h \simeq i, k \simeq i$ and $h \simeq j$; from 
the first, by symmetry, $i \simeq h$; so with the second, by transitivity, $k \simeq h$, and with the third, by transitivity again, $k \simeq j$; so $j \in \bar{k}$. This is impossible; reject the assumption: if $i \in \bar{h}$ and $i \in \bar{k}$, then $\overline{\mathrm{h}}=\overline{\mathrm{k}}$.

equality: For any $i$ and $j, j \simeq i$ iff $\bar{i}=\bar{j}$. Suppose $j \simeq i$; then $i \in \bar{j}$; but by self-membership, $i \in \bar{i}$; so by uniqueness, $\bar{i}=\bar{j}$. Suppose $\bar{i}=\bar{j}$; by self-membership, $i \in \bar{i}$; so $i \in \bar{j}$; so $j \simeq i$.

Now for the main lemma:

$\mathrm{D}$ is consistently specified. Suppose otherwise; then for some $s$ and $\bar{i}=\bar{j}, \bar{i} \in D\left(w_{s}\right)$ but $\bar{j} \notin D\left(w_{s}\right)$. So suppose $\bar{i}=\bar{j}$ and $\bar{i} \in D\left(w_{s}\right)$. If $i=j$, it is immediate that $\bar{j} \in D\left(w_{s}\right)$. So suppose $i \neq j$. Since $\bar{i} \in D\left(w_{s}\right)$, by construction, $\Gamma^{\prime \prime} \vdash_{\mathrm{Nhn} \alpha}^{*}\left(E a_{i}\right)_{s}$. And since $\bar{i}=\bar{j}$, by equality, $i \simeq j$; so, since $i$ and $j$ are distinct, for some $t, \Gamma^{\prime \prime} \vdash_{\text {Nhn } \alpha}^{*}\left(a_{i}=a_{j}\right)_{t}$; so by $=E$, $\Gamma^{\prime \prime} \vdash_{\mathrm{Nhn \alpha}}^{*}\left(\mathrm{Ea}_{\mathfrak{j}}\right)_{s}$; so by construction, $\overline{\mathfrak{j}} \in \mathrm{D}\left(w_{\mathrm{s}}\right)$.

$v_{w}\left(\mathrm{Q}^{\mathrm{n}}\right)$ is consistently specified. Suppose otherwise; then for some $s$ and $\langle\bar{h} \ldots \bar{k}\rangle=\langle\bar{d} \ldots \overline{\bar{l}}\rangle,\langle\bar{h} \ldots \bar{k}\rangle \in v_{w_{s}}\left(Q^{\mathfrak{n}}\right)$ but $\langle\overline{\mathrm{d}} \ldots \overline{\mathrm{l}}\rangle \notin v_{w_{s}}\left(\mathrm{Q}^{\mathfrak{n}}\right)$. So suppose $\langle\bar{h} \ldots \bar{k}\rangle=\langle\bar{d} \ldots \bar{l}\rangle$ and $\langle\bar{h} \ldots \bar{k}\rangle \in v_{w_{s}}\left(Q^{n}\right)$. If $h=d$ and $\ldots$ and $k=l$, it is immediate that $\langle\overline{\mathrm{d}} \ldots \overline{\mathrm{l}}\rangle \in v_{w_{s}}\left(\mathrm{Q}^{\mathrm{n}}\right)$. So suppose some $i$ in $h \ldots k$ is distinct from the corresponding $j$ in $d \ldots l$. Since $\langle\bar{h} \ldots \bar{i} \ldots \bar{k}\rangle \in v_{w_{s}}\left(Q^{n}\right)$, by construction, $\Gamma^{\prime \prime} \vdash_{\text {NFn } \alpha}^{*}\left(Q^{n} a_{h} \ldots a_{i} \ldots a_{k}\right)_{s}$. Since $\langle\bar{h} \ldots \bar{i} \ldots \bar{k}\rangle=\langle\bar{d} \ldots \bar{j} \ldots \bar{l}\rangle, \bar{i}=\bar{j}$; so by equality, $i \simeq j$; so since $i$ and $j$ are distinct, for some $t, \Gamma^{\prime \prime} \vdash_{\text {NFn } \alpha}^{*}\left(a_{i}=a_{j}\right)_{t}$; so by $=E, \Gamma^{\prime \prime} \vdash_{\text {NFn } \alpha}^{*}$ $\left(Q^{n} a_{h} \ldots a_{j} \ldots a_{k}\right)_{s}$; and similarly for other members that are distinct; so $\Gamma^{\prime \prime} \vdash_{N \text { Nh } \alpha}^{*}\left(Q^{n} a_{d} \ldots a_{j} \ldots a_{l}\right)_{s}$; so by construction, $\langle\bar{d} \ldots \bar{l}\rangle \in v_{w_{s}}\left(Q^{n}\right)$.

$v\left(a_{i}\right)$ is consistently specified - any constant $a_{i}$ is assigned to exactly one member of $U$. This follows immediately from self-membership and uniqueness.

L9.I4 $I=\langle W, U, D, R, P, v\rangle$ constructed as above is such that $\langle\bar{i} \ldots \bar{j}\rangle \in \bar{v}_{w_{s}}\left(Q^{n}\right)$ iff $\Gamma^{\prime \prime} \vdash_{\mathrm{N} n \mathrm{n} \alpha}^{*}\left(\overline{\mathrm{Q}^{\mathrm{n}} \mathrm{a}_{1} \ldots \mathrm{a}_{\mathrm{n}}}\right)_{s}$.

(i) Suppose $\langle\bar{i} \ldots \bar{j}\rangle \in \bar{v}_{\mathcal{w}_{s}}\left(\mathrm{Q}^{\mathfrak{n}}\right)$; then by the construction of $\bar{v}$, either $\langle\bar{i} \ldots \bar{j}\rangle \in v_{w_{s}}\left(Q^{n}\right)$, or for some $k$ in $i \ldots j, P\left(Q^{n}\right)_{k}=2$ and $\bar{k} \notin D\left(w_{s}\right)$. In the first case, by construction, $\Gamma^{\prime \prime} \vdash_{\text {NFn } \alpha}^{*}\left(Q^{n} a_{i} \ldots a_{j}\right)_{s}$; so by (D), it follows that $\Gamma^{\prime \prime} \vdash_{\mathrm{NFn} \alpha}^{*}\left(\overline{\mathrm{Q}^{n} a_{i} \ldots a_{j}}\right)_{s}$. In the second case, by construction, $E a_{k}$ is a conjunct of $P 2\left[Q^{n} a_{i} \ldots a_{j}\right]$ and $\Gamma^{\prime \prime} H_{N F n \alpha}^{*}\left(E a_{k}\right)_{s}$; however, if $\Gamma^{\prime \prime} \vdash_{\mathrm{Nhn} \alpha}^{*} \neg\left(\mathrm{Q}^{\mathrm{n}} \mathrm{a}_{i} \ldots \mathrm{a}_{\mathrm{j}}\right)_{s}$, then by $\mathrm{P}_{2} \mathrm{I}, \Gamma^{\prime \prime} \vdash_{\mathrm{Nhn} \alpha}^{*} \mathrm{P} 2\left[\mathrm{Q}^{\mathrm{n}} \mathrm{a}_{i} \ldots \mathrm{a}_{\mathrm{j}}\right]_{s}$, and by $\wedge \mathrm{E}, \Gamma^{\prime} \vdash_{\mathrm{Nhn} \alpha}^{*}\left(\mathrm{Ea}_{\mathrm{k}}\right)_{s}$; so we have $\Gamma^{\prime \prime} \nvdash_{\mathrm{NFn} \alpha}^{*}\left(\neg \mathrm{Q}^{\mathrm{n}} \mathrm{a}_{\mathrm{i}} \ldots \mathrm{a}_{\mathrm{j}}\right)_{s}$; but by L9.9, $\Gamma^{\prime \prime}$ is s-maximal; so $\Gamma^{\prime \prime} \vdash_{\mathrm{N} n \alpha}^{*}\left(\overline{\mathrm{Q}^{\mathfrak{n}} \mathrm{a}_{i} \ldots \mathrm{a}_{\mathrm{j}}}\right)_{s}$. So in either case, $\Gamma^{\prime \prime} \vdash_{\mathrm{NFn} \alpha}^{*}\left(\overline{\mathrm{Q}^{\mathrm{n}} \mathrm{a}_{i} \ldots \mathrm{a}_{\mathrm{j}}}\right)_{\mathrm{s}}$.

(iib) Suppose $\Gamma^{\prime \prime} \vdash_{\mathrm{Nhn \alpha}}^{*}\left(\overline{\mathrm{Q}^{\mathrm{n}} \mathrm{a}_{i} \ldots \mathrm{a}_{j}}\right)_{s}$. Either $\Gamma^{\prime \prime} \vdash_{\mathrm{NFn} \alpha}^{*} \mathrm{P} 2\left[\mathrm{Q}^{\mathrm{n}} \mathrm{a}_{i} \ldots \mathrm{a}_{\mathrm{j}}\right]_{s}$ or not. If $\Gamma^{\prime \prime} \vdash_{\mathrm{N} n \alpha}^{*} \mathrm{P} 2\left[\mathrm{Q}^{\mathrm{n}} \mathrm{a}_{\mathfrak{i}} \ldots \mathrm{a}_{\mathrm{j}}\right]_{s}$, by $\mathrm{P} 2 \mathrm{E}, \Gamma^{\prime \prime} \vdash_{\mathrm{N} n \alpha}^{*}\left(\mathrm{Q}^{\mathrm{n}} \mathrm{a}_{\mathfrak{i}} \ldots \mathrm{a}_{\mathfrak{j}}\right)_{s}$; so by construction, $\langle\bar{i} \ldots \bar{j}\rangle \in v_{w_{s}}\left(Q^{\mathfrak{n}}\right)$; so by the construction of $\bar{v},\langle\bar{i} \ldots \bar{j}\rangle \in$ 
$\bar{v}_{w_{s}}\left(Q^{n}\right)$. If $\Gamma^{\prime \prime} \nvdash_{N \text { Nn } \alpha}^{*} P 2\left[Q^{n} a_{i} \ldots a_{j}\right]_{s}$, there is some $k$ in $i \ldots j$ such that $\mathrm{P}\left(\mathrm{Q}^{\mathrm{n}}\right)_{k}=2$, and $\Gamma^{\prime \prime} \nvdash_{\mathrm{N} F \mathrm{n} \alpha}^{*}\left(\mathrm{Ea}_{\mathrm{k}}\right)_{s}$; from the latter, by construction, $\overline{\mathrm{k}} \notin \mathrm{D}\left(w_{s}\right)$; so by the construction of $\bar{v},\langle\bar{i} \ldots \bar{j}\rangle \in \bar{v}_{w_{s}}\left(\mathrm{Q}^{\mathfrak{n}}\right)$. So in either case, $\langle\bar{i} \ldots \bar{j}\rangle \in \bar{v}_{w_{s}}\left(Q^{\mathfrak{n}}\right)$.

L9.I5 If $\Gamma_{0}^{\prime}$ is consistent then for $\langle\mathrm{W}, \mathrm{U}, \mathrm{D}, \mathrm{R}, \mathrm{P}, v\rangle$ constructed as above, any sentence $/ A /$ in $\mathcal{L}$, and for any s included in $\Gamma^{\prime \prime}, h_{w_{s}}(/ A /)=1$ iff $\Gamma^{\prime \prime} \vdash_{\text {NFn } \alpha}^{*}$ $/ \mathrm{A} / \mathrm{s}$.

Suppose $\Gamma_{0}^{\prime}$ is consistent and $s$ is included in $\Gamma^{\prime \prime}$. By L9.9, $\Gamma^{\prime \prime}$ is smaximal. By L9.II and L9.I2, $\Gamma^{\prime \prime}$ is consistent and a scapegoat set for $\square$ and $\forall$. Now by induction on the number of operators in $/ A / s$,

Basis: If $/ \mathrm{A} / \mathrm{s}$ has no operators, then it follows that it is is an atomic of the sort $/ Q^{n} a_{i} \ldots a_{j} / . h_{w_{s}}(/ A /)=1$ iff $h_{w_{s}}\left(/ Q^{n} a_{i} \ldots a_{j} /\right)=$ 1; iff, for arbitrary $\delta, h_{w_{s}}\left(/ Q^{n} a_{i} \ldots a_{j} /\right) / / \delta=1$; by $H F(R)$, iff $\left\langle\Delta\left(a_{i}\right) \ldots \Delta\left(a_{j}\right)\right\rangle \in / v^{\prime} w_{s}\left(Q^{n}\right) ;$ iff $\langle\bar{i} \ldots \bar{j}\rangle \in / v^{\prime} / w_{s}\left(Q^{n}\right)$; by construction and L9.I4, iff $\Gamma^{\prime \prime} \vdash_{\mathrm{NFn} \alpha}^{*}\left(/ Q^{n} a_{i} \ldots a_{j} /\right)_{s} ;$ iff $\Gamma^{\prime \prime} \vdash_{N F n \alpha}^{*}$ $/ \mathrm{A} / \mathrm{s}$.

Assp: For any $i, 0 \leqslant i<k$, if $/ A / s$ has $i$ operators, then $h_{w_{s}}(/ A /)=1$ iff $\Gamma^{\prime \prime} \vdash_{\mathrm{N} F \mathrm{n} \alpha}^{*} / \mathrm{A} / \mathrm{s}$.

Show: If $/ A / s$ has $k$ operators, then $h_{w_{s}}(/ A /)=1 \mathrm{iff} \Gamma^{\prime \prime} \vdash_{\text {Nhn } \alpha}^{*} / A / s$. If $/ A / s$ has $k$ operators, then it is of the form $/ \neg P / s, / P \wedge Q / s$, $/ \square \mathrm{P} / \mathrm{s}$ or $/ \forall \mathrm{xP} / \mathrm{s}$, where $\mathrm{P}$ and $\mathrm{Q}$ have $<\mathrm{k}$ operators.

$(\neg) / A / s$ is $/ \neg P / s$. (i) Suppose $h_{w_{s}}(/ A /)=1$; then $h_{w_{s}}(\neg P /)=1$; so for arbitrary $\delta, h_{w_{s}}(/ \neg \mathrm{P} /) / / \delta=1$; so by $\mathrm{HF}(\neg), h_{w_{s}}(\backslash \mathrm{P} \backslash) / / \delta$ $=0$; so, $h_{w_{s}}(\backslash P \backslash)=0$; so by assumption, $\Gamma^{\prime \prime} \nvdash_{\mathrm{NFn} \alpha}^{*} \backslash P \backslash_{s}$; so by smaximality, $\Gamma^{\prime \prime} \vdash_{\mathrm{NFn} \alpha}^{*} / \neg \mathrm{P} / \mathrm{s}$, where this is to say, $\Gamma^{\prime \prime} \vdash_{\mathrm{NFn \alpha}}^{*} / \mathrm{A} / \mathrm{s}$. (ii) Suppose $\Gamma^{\prime \prime} \vdash_{\mathrm{Nhr} \alpha}^{*} / \mathrm{A} / s$; then $\Gamma^{\prime \prime} \vdash_{\mathrm{NFn} \alpha}^{*} / \neg \mathrm{P} / s$; so by consistency, $\Gamma^{\prime \prime} \nvdash_{\mathrm{NFn} \alpha}^{*} \backslash P \backslash_{s}$; so by assumption, $h_{w_{s}}(\backslash P \backslash)=0$; so there is a $\delta$ such that $h_{w_{s}}(\backslash P \backslash) / / \delta=0$; so by $\operatorname{HF}(\neg), h_{w_{s}}(/ \neg P /) / / \delta=1$; and since $/ A / s$ has no free variables, by $L 9.2, h_{w_{s}}(\neg P /)=1$, where this is to say, $h_{w_{s}}(/ A /)=1$. So $h_{w_{s}}(/ A /)=1$ iff $\Gamma^{\prime \prime} \vdash_{\text {NFn } \alpha}^{*} / A / s$.

$(\wedge)$

( $\square$ ) $/ A / s$ is $/ \square P / s$. (i) Suppose $h_{w_{s}}(/ A /)=1$, but $\Gamma^{\prime \prime} \nvdash_{\text {NFn } \alpha}^{*} / A / s$; then $h_{w_{s}}(/ \square \mathrm{P} /)=1$, but $\Gamma^{\prime \prime} H_{\mathrm{Nhn} \alpha}^{*} / \square \mathrm{P} / \mathrm{s}$. From the latter, by maximality, $\Gamma^{\prime \prime} \vdash_{\mathrm{NFn} \alpha}^{*} \backslash \neg \square \mathrm{P} \backslash_{s}$; so, since $\Gamma^{\prime \prime}$ is a scapegoat set for $\square$, there is some $t$ such that $\Gamma^{\prime \prime} \vdash_{\mathrm{N} \text { n } \alpha}^{*}$ s.t and $\Gamma^{\prime \prime} \vdash_{\mathrm{NFn} \alpha}^{*} \backslash \neg \mathrm{P} \backslash_{\mathrm{t}}$; from the first of these, by construction, $\left\langle w_{s}, w_{t}\right\rangle \in R$; and from the second, by consistency, $\Gamma^{\prime \prime} \nvdash_{\mathrm{N} F \alpha}^{*} / \mathrm{P} / \mathrm{t}$; so by assumption, $h_{w_{t}}(/ \mathrm{P} /)=0$; so for some $\delta, h_{w_{t}}(/ P /) / / \delta=0$; so by $\operatorname{HF}(\square), h_{w_{s}}(/ \square \mathrm{P} /) / / \delta=0$; so $h_{w_{s}}(/ \square \mathrm{P} /)=0$. This is impossible; reject the assumption: if $h_{w_{s}}(/ A /)=1$, then $\Gamma^{\prime \prime} \vdash_{\text {N } n \text { n }}^{*} / A / s$. 
(ii) Suppose $\Gamma^{\prime \prime} \vdash_{\mathrm{Nhn} \alpha}^{*} / \mathrm{A} / \mathrm{s}$, but $h_{w_{s}}(/ \mathrm{A} /)=0$; then $\Gamma^{\prime \prime} \vdash_{\mathrm{Nhn \alpha}}^{*}$ $/ \square \mathrm{P} / \mathrm{s}$, but $h_{w_{s}}(/ \square \mathrm{P} /)=0$. From the latter, there is a $\delta$ such that $h_{w_{s}}(/ \square \mathrm{P} /) / / \delta=0$; so by $\mathrm{HF}(\square)$, there is a $w_{\mathrm{t}} \in \mathrm{W}$ such that $w_{\mathrm{s}} \mathrm{R} w_{\mathrm{t}}$ and $h_{w_{\mathrm{t}}}(/ \mathrm{P} /) / / \delta=0$; so $h_{w_{\mathrm{t}}}(/ \mathrm{P} /)=0$; so by assumption, $\Gamma^{\prime \prime} H_{\mathrm{N} h \alpha}^{*} / \mathrm{P} / \mathrm{t}$; but since $w_{\mathrm{s}} R w_{\mathrm{t}}$, by construction, $\Gamma^{\prime \prime} \vdash_{\mathrm{Nhn} \alpha}^{*}$ s.t; so by $\square \mathrm{E}, \Gamma^{\prime \prime} \vdash_{\mathrm{N} n \alpha}^{*} / \mathrm{P} / \mathrm{t}$. This is impossible; reject the assumption: if $\Gamma^{\prime \prime} \vdash_{\mathrm{NFn} \alpha}^{*} / A / s$, then $h_{w_{s}}(/ A /)=1$. So $h_{w_{s}}(/ A /)=1$ iff $\Gamma^{\prime \prime} \vdash_{\mathrm{N} \text { rn } \alpha}^{*} / \mathrm{A} / \mathrm{s}$.

( $\forall) / A / s$ is $/ \forall x P / s$. (i) Suppose $h_{w_{s}}(/ A /)=1$, but $\Gamma^{\prime \prime} H_{N F n \alpha}^{*} / A / s$; then $h_{w_{s}}(/ \forall x \mathrm{P} /)=1$, but $\Gamma^{\prime \prime} \nvdash_{\mathrm{N} r \alpha \alpha}^{*} / \forall x \mathrm{P} / \mathrm{s}$. From the latter, by maximality, $\Gamma^{\prime \prime} \vdash_{\mathrm{NFn} \alpha}^{*} \backslash \neg \forall x \mathrm{P} \backslash_{s}$; so, since $\Gamma^{\prime \prime}$ is a scapegoat set for $\forall$, there is some $a_{i}$ such that $\Gamma^{\prime \prime} \vdash_{\mathrm{NFn} \alpha}^{*}\left(E a_{i}\right)_{s}$ and $\Gamma^{\prime \prime} \vdash_{\mathrm{Nhn} \alpha}^{*} \backslash \neg \mathrm{P}^{\mathrm{X}} / \mathrm{a}_{i} \backslash_{s}$; from the first of these, by construction, $\bar{i} \in \mathrm{D}\left(w_{s}\right)$; and from the second, by consistency, $\Gamma^{\prime \prime} \nvdash_{N \hbar n \alpha}^{*} / \mathrm{P}^{\mathrm{x}} / \mathrm{a}_{\mathrm{i}} / s$; so by assumption, $h_{w_{s}}\left(/ \mathrm{P}^{\mathrm{x}} / \mathrm{a}_{\mathrm{i}} /\right)=0$; so for some $\delta, \mathrm{h}_{w_{s}}\left(/ \mathrm{P}^{\mathrm{x}} / \mathrm{a}_{\mathrm{i}} /\right) / / \delta=0$; but $v\left(\mathrm{a}_{\mathrm{i}}\right)=$ $\bar{i}$; so $\Delta\left(a_{i}\right)=\bar{i}$; so by L9.4, $h_{w(s)}(/ P /) / / \delta[x \mid \bar{i}]=0$; so by $\operatorname{HF}(\forall)$, $h_{w_{s}}(/ \forall x \mathrm{P} /) / / \delta=0$; so $h_{w_{s}}(/ \forall x \mathrm{P} /)=0$. This is impossible; reject the assumption: if $h_{w_{s}}(/ A /)=1$, then $\Gamma^{\prime \prime} \vdash_{\mathrm{Nhn \alpha}}^{*} / A / s$.

(ii) Suppose $\Gamma^{\prime \prime} \vdash_{\mathrm{NFn} \alpha}^{*} / A / s$, but $h_{w_{s}}(/ A /)=0$; then $\Gamma^{\prime \prime} \vdash_{\mathrm{NFn} \alpha}^{*}$ $/ \forall x \mathrm{P} /{ }_{s}$, but $h_{w_{s}}(/ \forall x \mathrm{P} /)=0$. From the latter, there is a $\delta$ such that $h_{w_{s}}(/ \forall x \mathrm{P} /) / / \delta=0$; so by $\mathrm{HF}(\forall)$, there is some $\bar{i} \in \mathrm{D}\left(w_{s}\right)$ such that $h_{w_{s}}(/ P /) / / \delta[x \mid \bar{i}]=0$. Since $\bar{i} \in D\left(w_{s}\right)$, by construction, $\Gamma^{\prime \prime} \vdash_{N \text { n } \alpha}^{*}\left(E a_{i}\right)_{s}$. And since $v\left(a_{i}\right)=\bar{i}, \Delta\left(a_{i}\right)=\bar{i}$; so by L9.4, $h_{w_{s}}\left(/ \mathrm{P}^{\mathrm{x}} / \mathrm{a}_{\mathrm{i}} /\right) / / \delta=0$; so $h_{w_{s}}\left(/ \mathrm{P}^{\mathrm{x}} / \mathrm{a}_{\mathrm{i}} /\right)=0$; so by assumption, $\Gamma^{\prime \prime} \vdash_{\mathrm{NFn} \alpha}^{*} / \mathrm{P}^{\mathrm{x}} / \mathrm{a}_{\mathrm{i}} / s$. But since $\Gamma^{\prime \prime} \vdash_{\mathrm{NFn} \alpha}^{*} / \forall \mathrm{xP} / \mathrm{s}$ and $\Gamma^{\prime \prime} \vdash_{\mathrm{NFn} \alpha}^{*}\left(E a_{i}\right)_{s}$, by $\forall \mathrm{E}, \Gamma^{\prime \prime} \vdash_{\mathrm{N} n \alpha}^{*} / \mathrm{P}^{\mathrm{x}} / \mathrm{a}_{\mathrm{i}} / s$. This is impossible; reject the assumption: if $\Gamma^{\prime \prime} \vdash_{\mathrm{Nhn \alpha}}^{*} / A / s$, then $h_{w_{s}}(/ A /)=1$. So $h_{w_{s}}(/ A /)=1$ iff $\Gamma^{\prime \prime} \vdash_{\mathrm{NFn} \alpha}^{*} / \mathrm{A} / \mathrm{s}$.

For any $A_{s}, h_{w_{s}}(/ A /)=1$ iff $\Gamma^{\prime \prime} \vdash_{N F n \alpha}^{*} / A / s$.

L9.I 6 If $\Gamma_{0}^{\prime}$ is consistent, then $\langle W, U, D, R, P, v\rangle$ constructed as above is an $F n \alpha$ interpretation.

Suppose $\Gamma_{0}^{\prime}$ is consistent.

(EP) Suppose $\langle\bar{i} \ldots \bar{j}\rangle \in v_{w_{s}}\left(Q^{n}\right)$ and for some $k$ in $i \ldots j, P\left(Q^{n}\right)_{k} \geqslant 1$. From the former, by construction, $\Gamma^{\prime \prime} \vdash_{\text {Nhn } \alpha}^{*}\left(Q^{n} a_{i} \ldots a_{j}\right)_{s}$; so by PII, $\Gamma^{\prime \prime} \vdash_{\text {Nfn } \alpha}^{*} P 1\left[Q^{n} a_{i} \ldots a_{j}\right]_{s}$. But since since $P\left(Q^{n}\right)_{k} \geqslant 1$, by construction, $E a_{k}$ is a conjunct of $P 1\left[Q^{n} a_{i} \ldots a_{j}\right]$; so with $\wedge \mathrm{E}, \Gamma^{\prime \prime} \vdash_{\mathrm{NFn} \alpha}^{*}\left(\mathrm{Ea}_{\mathrm{k}}\right)_{s}$; so by construction, $\overline{\mathrm{k}} \in \mathrm{D}\left(w_{\mathrm{s}}\right)$, and $\mathrm{EP}$ is satisfied.

(E) By construction, $\bar{i} \in v_{w_{s}}(E)$ iff $\Gamma^{\prime \prime} \vdash_{\text {NFn } \alpha}^{*}\left(E a_{i}\right)_{s}$; by construction again, iff $\bar{i} \in \mathrm{D}\left(w_{s}\right)$. 
(=) Suppose $\mathrm{P}(=)=\langle 0,0\rangle$. (i) Suppose $\langle\bar{i}, \bar{j}\rangle \in v_{w_{s}}(=)$; then by construction, $\Gamma^{\prime \prime} \vdash_{\text {Nfrn }}^{*}\left(a_{i}=a_{j}\right)_{s}$; so $i \simeq j$; so by equality $\bar{i}=\bar{j}$. (ii) Suppose $\bar{i}=\bar{j}$. Since $P(=)=\langle 0,0\rangle$ it is trivial that $\Gamma^{\prime \prime} \vdash_{\text {Nhn } \alpha}^{*}$ P1 $\left[a_{i}=a_{i}\right]_{s}$; so by $(=I), \Gamma^{\prime \prime} \vdash_{\text {Nhn } \alpha}^{*}\left(a_{i}=a_{i}\right)_{s}$. And since $\bar{i}=\bar{j}$, by equality, $i \simeq j$; so either $i=j$ or for some $t, \Gamma^{\prime \prime} \vdash_{\text {Nhn } \alpha}^{*}\left(a_{i}=a_{j}\right)_{t}$. In the first case, since $\Gamma^{\prime \prime} \vdash_{\text {Nhn } \alpha}\left(a_{i}=a_{i}\right)_{s}, \Gamma^{\prime \prime} \vdash_{N \text { Nh } \alpha}\left(a_{i}=a_{j}\right)_{s}$ so that, by construction, $\langle\bar{i}, \bar{j}\rangle \in v_{w_{s}}(=)$. In the second case, by $(=\mathrm{E}), \Gamma^{\prime \prime} \vdash_{\mathrm{NFn} \alpha}^{*}\left(a_{\mathfrak{i}}=a_{\mathfrak{j}}\right)_{s}$; and by construction, $\langle\bar{i}, \bar{j}\rangle \in v_{w_{s}}(=)$. In either case, then, $\langle\bar{i}, \bar{j}\rangle \in v_{w_{s}}(=)$. So the interpretation of $(=)$ is as it should be.

Suppose $\mathrm{P}(=) \neq\langle 0,0\rangle$. (i) Suppose $\langle\overline{\mathrm{i}}, \bar{j}\rangle \in v_{w_{s}}(=)$; then by construction, $\Gamma^{\prime \prime} \vdash_{\text {Nhn } \alpha}^{*}\left(a_{i}=a_{j}\right)_{s}$; so $i \simeq j$; so by equality, $\bar{i}=\bar{j}$. And since $\Gamma^{\prime \prime} \vdash_{\mathrm{NFn} \alpha}^{*}\left(a_{i}=a_{j}\right)_{s}$, by PII, $\Gamma^{\prime \prime} \vdash_{\text {NFr } \alpha}^{*} P 1\left[a_{i}=a_{j}\right]_{s}$; so since $\mathrm{P}(=)$ has some member $\neq 0$, with $\wedge \mathrm{E}$, either $\Gamma^{\prime \prime} \vdash_{\mathrm{NFn} \alpha}^{*}\left(\mathrm{Ea}_{i}\right)_{s}$ or $\Gamma^{\prime \prime} \vdash_{\mathrm{NFn} \alpha}^{*}\left(E a_{\mathfrak{j}}\right)_{s}$; so by construction, $\bar{i} \in \mathrm{D}\left(w_{s}\right)$ or $\bar{j} \in \mathrm{D}\left(w_{s}\right)$; so, since $\bar{i}=\bar{j}, \bar{i} \in D\left(w_{s}\right)$. (ii) Suppose $\bar{i}=\bar{j}$ and $\bar{i} \in D\left(w_{s}\right)$. From the latter, $\Gamma^{\prime \prime} \vdash_{\mathrm{N} \text { hn } \alpha}^{*}\left(E a_{i}\right)_{s}$; so for any version of $\mathrm{P} 1\left[a_{i}=a_{i}\right]$, $\Gamma^{\prime \prime} \vdash_{\mathrm{NFn} \alpha}^{*} \mathrm{P} 1\left[a_{i}=a_{i}\right]_{s}$; so by $(=) \mathrm{I}, \Gamma^{\prime \prime} \vdash_{\mathrm{NFn} \alpha}^{*}\left(a_{i}=a_{i}\right)_{s}$. From the former, by equality, $i \simeq j$; so either $i=j$ or for some $t$, $\Gamma^{\prime \prime} \vdash_{\text {Nhn } \alpha}^{*}\left(a_{i}=a_{j}\right)_{t}$. In the first case, since $\Gamma^{\prime \prime} \vdash_{\text {NFn } \alpha}\left(a_{i}=a_{i}\right)_{s}$, $\Gamma^{\prime \prime} \vdash_{\text {NFn } \alpha}\left(a_{i}=a_{j}\right)_{s}$ so that, by construction, $\langle\bar{i}, \bar{j}\rangle \in v_{w_{s}}(=)$. In the second case, by $(=) \mathrm{E}, \Gamma^{\prime \prime} \vdash_{\mathrm{NFn} \alpha}^{*}\left(a_{i}=a_{j}\right)_{s}$; and by construction, $\langle\bar{i}, \bar{j}\rangle \in v_{w_{s}}(=)$. In either case, then, $\langle\bar{i}, \bar{j}\rangle \in v_{w_{s}}(=)$. So the interpretation of $(=)$ is as it should be.

( $\eta$ Suppose $\alpha$ includes condition $\eta$ and $w_{s} \in W$. Then, by construction, $s$ is a subscript in $\Gamma^{\prime}$; so by reasoning as follows,

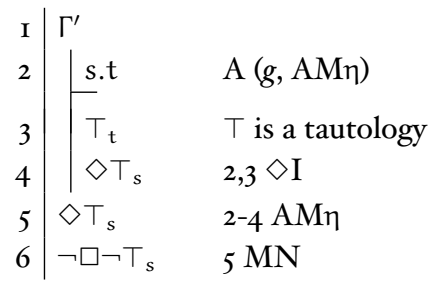

$\Gamma^{\prime} \vdash_{\mathrm{NFn} \alpha}^{*} \neg \square \neg \top_{s}$; but by L9.I2, $\Gamma^{\prime}$ is a scapegoat set for $\square$; so there is a $t$ such that $\Gamma^{\prime} \vdash_{\text {NFn }}^{*}$ s.t; so by construction, $\left\langle w_{s}, w_{t}\right\rangle \in R$ and $\eta$ is satisfied.

( $\rho$ ) Suppose $\alpha$ includes condition $\rho$ and $w_{s} \in W$. Then by construction, $s$ is a subscript in $\Gamma^{\prime}$; so by $(\mathrm{AM} \rho), \Gamma^{\prime} \vdash_{\mathrm{N} F \mathrm{n} \alpha}^{*}$ s.s; so by construction, $\left\langle w_{s}, w_{s}\right\rangle \in R$ and $\rho$ is satisfied.

( $\sigma)$ Suppose $\alpha$ includes condition $\sigma$ and $\left\langle w_{s}, w_{t}\right\rangle \in R$. Then by construction, $\Gamma^{\prime} \vdash_{\mathrm{NFn} \alpha}^{*}$ s.t so by $(\mathrm{AM} \sigma), \Gamma^{\prime} \vdash_{\mathrm{NFn} \alpha}^{*}$ t.s; so by construction, $\left\langle w_{\mathrm{t}}, w_{\mathrm{s}}\right\rangle \in R$ and $\sigma$ is satisfied.

( $\tau$ ) Suppose $\alpha$ includes condition $\tau$ and $\left\langle w_{s}, w_{\mathfrak{t}}\right\rangle,\left\langle w_{\mathfrak{t}}, w_{\mathfrak{u}}\right\rangle \in R$. Then 
by construction, $\Gamma^{\prime} \vdash_{\mathrm{NFn} \alpha}^{*}$ s.t and $\Gamma^{\prime} \vdash_{\mathrm{NFn} \alpha}^{*}$ t.u; so by $(\mathrm{AM} \tau), \Gamma^{\prime} \vdash_{\mathrm{NFn} \alpha}^{*}$ s.u; so by construction, $\left\langle w_{s}, w_{u}\right\rangle \in R$ and $\tau$ is satisfied.

MAP For any $w_{s} \in W$, set $m(s)=w_{s}$; otherwise $m(s)$ is arbitrary. And let $\mathrm{I}^{\prime}=\left\langle\mathrm{W}, \mathrm{U}, \mathrm{D}, \mathrm{R}, \mathrm{P}, v^{\prime}\right\rangle$ be like I except without assignments to constants not in the original language. Clearly $\mathrm{I}^{\prime}$ is an interpretatio for our our original language, and remains an $F n \alpha$ interpretation.

L9.I 7 If $\Gamma_{0}$ is consistent, then $h_{m}^{\prime}\left(\Gamma_{0}\right)=1$.

Suppose $\Gamma_{0}$ is consistent and $/ A / 0 \in \Gamma_{0}$; then $/ A / 0 \in \Gamma_{0}^{\prime}$ and by L9.7, $\Gamma_{0}^{\prime}$ is consistent; then by construction, $/ A /_{0} \in \Gamma^{\prime \prime}$; so $\Gamma^{\prime \prime} \vdash_{\mathrm{Nhn \alpha}}^{*} / A /{ }_{0}$; so by $L 9.15, h_{w_{0}}(/ A /)=1$; but $/ A /$ is a sentence of the original language without extra constants; so by the corollary to L9.3, $h_{w_{0}}^{\prime}(/ A /)=1$. And similarly for any $/ A /_{0} \in \Gamma_{0}$. But $m(0)=w_{0} ;$ so $h_{m}^{\prime}\left(\Gamma_{0}\right)=1$.

Main result: Suppose $\Gamma \models_{\text {nn } \alpha} / A /$ but $\Gamma H_{\mathrm{NFn} \alpha} / A /$. Then $\Gamma_{0} \models_{\text {m } \alpha}^{*} / A / 0$ but $\Gamma_{0} H_{\mathrm{NFn} \alpha}^{*}$ $/ A / 0$. By $(D N)$, if $\Gamma_{0} \vdash_{\mathrm{NFn} \alpha}^{*} / \neg \neg A / 0$, then $\Gamma_{0} \vdash_{\mathrm{NFn} \alpha}^{*} / A / 0$; so $\Gamma_{0} \nvdash_{\mathrm{NFn} \alpha}^{*} / \neg \neg A / 0$; so by L9.5, $\Gamma_{0} \cup\left\{\backslash \neg A \backslash_{0}\right\}$ is consistent; so by L9.I6 and L9.I 7, there is an Fn $\alpha$ interpretation $\mathrm{I}^{\prime}=\left\langle\mathrm{W}, \mathrm{U}, \mathrm{D}, \mathrm{R}, \mathrm{P}, v^{\prime}\right\rangle_{\mathrm{m}}$ constructed as above such that $h_{\mathrm{m}}^{\prime}\left(\Gamma_{0} \cup\right.$ $\left.\left\{\backslash \neg A \backslash_{0}\right\}\right)=1$; so $h_{m(0)}^{\prime}(\backslash \neg A \backslash)=1$; so for any $\delta, h_{m(0)}^{\prime}(\backslash \neg A \backslash) / / \delta=1$; so by $\mathrm{HF}(\neg), h_{m(0)}^{\prime}(/ A /) / / \delta=0$; so $h_{m(0)}^{\prime}(/ A /)=0$; so $h_{m}^{\prime}\left(\Gamma_{0}\right)=1$ and $h_{m(0)}^{\prime}(A)=$ 0 ; so by $\mathrm{VFN}_{\mathrm{N}} \alpha^{*}, \Gamma_{0} \nvdash_{\mathrm{n} \alpha}^{*} / \mathrm{A} / 0$. This is impossible; reject the assumption: if $\Gamma \models_{\text {nn } \alpha} / \mathrm{A} /$, then $\Gamma \vdash_{\mathrm{N} \text { (n } \alpha} / \mathrm{A} /$.

\section{REFERENCES}

[I] Bergmann, Moor and Nelson. The Logic Book 4th Ed. McGraw-Hill, New York, 2004 .

[2] Fitting and Mendelsohn. First-Order Modal Logic. Kluwer Academic Publishers, Boston, I999.

[3] James Garson. "Quantification in Modal Logic." In Handbook of Philosophical Logic II. Ed. Gabbay and Guenthner. Kluwer Academic Publishers, Boston, 1984.

[4] Graham Priest. An Introduction to Non-Classical Logic. Cambridge University Press, Cambridge, 200I.

[5] Alvin Plantinga. "Actualism and Possible Worlds." Reprint in Essays in the Metaphysics of Modality. Ed. Davidson. Oxford University Press, New York, 2003.

[6] Alvin Plantinga. "World and Essence." Reprint in Essays in the Metaphysics of Modality. Ed. Davidson. Oxford University Press, New York, 2003. 
[7] W.V. Quine. "Reference and Modality." Revised version in his, From a Logical Point of View, 2nd edition. Harvard University Press, Cambridge, I980.

[8] Greg Restall. "Four-Valued Semantics for Relevant Logics (And Some of Their Rivals).” Journal of Philosophical Logic 24 (1995): 139-160.

[9] Richard Routley. "The American Plan Completed: Alternative Classical-Style Semantics, Without Stars, for Relevant and Paraconsistent Logics." Studia Logica 43 (1984): I3I-158.

[Io] Tony Roy. "Things and De Re Modality." Noûs 34 (2000): 56-84.

[II] Tony Roy. "Making Sense of Relevant Semantics." Unpublished, but available at

http://cal.csusb.edu/Faculty/Philosophy/roy/msrs-paper.pdf.

[12] Tony Roy. Symbolic Logic: An Accessible Introduction to Serious Mathematical Logic Unpublished, but available at http://cal.csusb.edu/Faculty/Philosophy/roy/int-ml.htm.

[13] Peter Woodruff. "Logic and Truth Value Gaps.” In Philosophical Problems in Logic. Ed. Lambert. D. Reidel, Dordrecht, 1970. 
The Australasian fournal of Logic (ISSN I448-5O52) disseminates articles that significantly advance the study of logic, in its mathematical, philosophical or computational guises. The scope of the journal includes all areas of logic, both pure and applied to topics in philosophy, mathematics, computation, linguistics and the other sciences.

Articles appearing in the journal have been carefully and critically refereed under the responsibility of members of the Editorial Board. Only papers judged to be both significant and excellent are accepted for publication.

The journal is freely available at the journal website at

$$
\text { http://www ·philosophy · unimelb . edu . au/ajl/. }
$$

All issues of the journal are archived electronically at the journal website.

SUBSCRIPTIONS Individuals may subscribe to the journal by sending an email, including a full name, an institutional affiliation and an email address to the managing editor at ajl-editors@unimelb. edu . au. Subscribers will receive email abstracts of accepted papers to an address of their choice. For institutional subscription, please email the managing editor at ajl-editors@unimelb.edu.au.

Complete published papers may be downloaded at the journal's website at http://www.philosophy . unimelb.edu.au/ajl/. The journal currently publishes in pdf format.

Submission The journal accepts submissions of papers electronically. To submit an article for publication, send the ${ }^{\Delta} \mathrm{T}_{\mathrm{E}} \mathrm{X}$ source of a submission to a member of the editorial board. For a current list of the editorial board, consult the website.

The copyright of each article remains with the author or authors of that article. 